\title{
QUALIFICATION OF THE SECOND ICS-3000 ION CHROMATOGRAPH FOR USE AT THE DEFENSE WASTE PROCESSING FACILITY
}

R.N. Mahannah

T.B. Edwards

October 2009

Applied Computational Engineering and Statistics Savannah River National Laboratory

Aiken, SC 29808

This document was prepared in conjunction with work accomplished under Contract No. DE-AC09-08SR22470 with the U.S. Department of Energy. 
SRNL-STI-2009-00686

Revision 0

\section{DISCLAIMER}

This work was prepared under an agreement with and funded by the U.S. Government. Neither the U.S. Government or its employees, nor any of its contractors, subcontractors or their employees, makes any express or implied: 1. warranty or assumes any legal liability for the accuracy, completeness, or for the use or results of such use of any information, product, or process disclosed; or 2. representation that such use or results of such use would not infringe privately owned rights; or 3. endorsement or recommendation of any specifically identified commercial product, process, or service. Any views and opinions of authors expressed in this work do not necessarily state or reflect those of the United States Government, or its contractors, or subcontractors.

This document was prepared in conjunction with work accomplished under Contract No. DE-AC09-08SR22470 with the U.S. Department of Energy. 
Keywords: ICS-3000,statistics, anions

Retention: permanent

\section{QUALIFICATION OF THE SECOND ICS-3000 ION CHROMATOGRAPH FOR USE AT THE DEFENSE WASTE PROCESSING FACILITY}

R.N. Mahannah

T.B. Edwards

October 2009

Applied Computational Engineering and Statistics Savannah River National Laboratory

Aiken, SC 29808

This document was prepared in conjunction with work accomplished under Contract No. DE-AC09-08SR22470 with the U.S. Department of Energy.

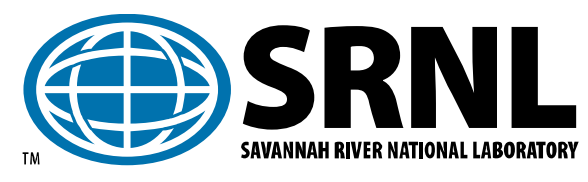


SRNL-STI-2009-00686

Revision 0

\section{REVIEWS AND APPROVALS}

\section{AUTHORS:}

R. N. Mahannah, Waste Laboratory Services

Date

Savannah River Remediation

T. B. Edwards, Applied Computational Engineering and Statistics

Date

Savannah River National Laboratory

\section{TECHNICAL REVIEWERS:}

D. R. Best, Process Technology Programs

Date

Savannah River National Laboratory

E. P. Shine, Applied Computational Engineering and Statistics Date

Savannah River National Laboratory

\section{APPROVERS:}

P. L. Lee, Manager, Applied Computational Engineering and Statistics Date Savannah River National Laboratory

C. C. Herman, Manager, Process Technology Programs Date Savannah River National Laboratory

S. L. Marra, Manager,

Date

Environmental \& Chemical Process Technology Research Programs

Savannah River National Laboratory

A. B. Osteen, Manager, Waste Laboratory Services

Date

Savannah River Remediation

J.E. Occhipinti, Manager, Waste Solidification Engineering

Date

Savannah River Remediation 


\section{EXECUTIVE SUMMARY}

The ICS-3000 Ion Chromatography (IC) system installed in 221-S M-14 has been qualified for use. The qualification testing was a head to head comparison of the second ICS-3000 with the initial ICS3000 system that was installed in 221-S M-13. The crosscheck work included standards for instrument calibration and calibration verifications and standards for individual anion analysis, where the standards were traceable back to the National Institute of Standards and Technology (NIST). In addition the crosscheck work included the analysis of simulated Sludge Receipt and Adjustment Tank (SRAT) Receipt, SRAT Product, and Slurry Mix Evaporator (SME) samples, along with radioactive Sludge Batch 5 material from the SRAT and SME tanks. 


\section{TABLE OF CONTENTS}

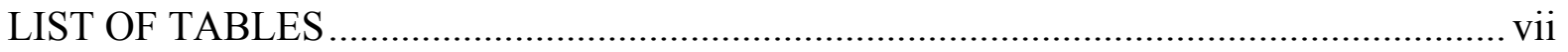

LIST OF ABBREVIATIONS ............................................................................. viii

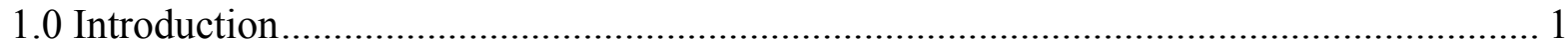

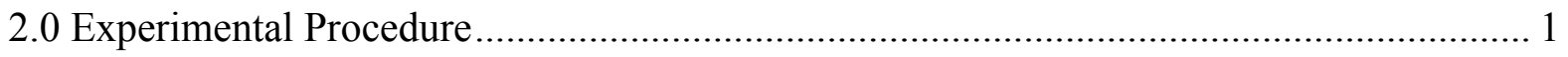

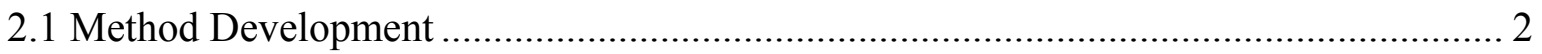

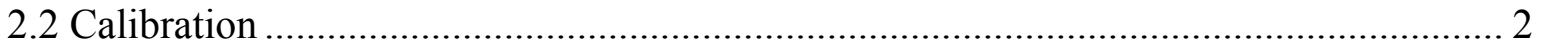

2.3 Sludge Sample Analysis .................................................................................... 2

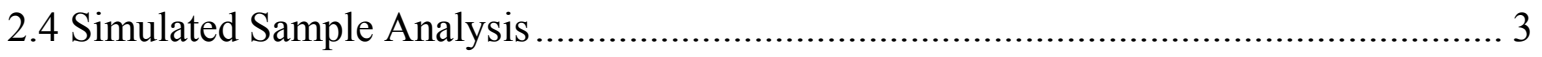

2.5 Analysis of Calibration Standards as Samples ...................................................... 3

2.6 Analysis of Standards Prepared from Dried Salts ................................................ 3

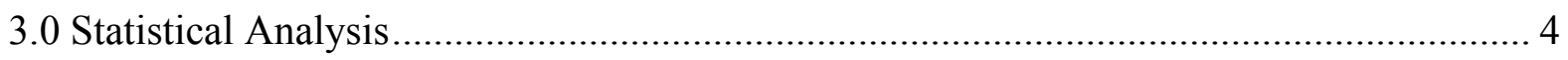

3.1 Preliminary Comparisons Using Standards ............................................................ 4

3.2 Full Set of Crosscheck Sample Results ......................................................................... 5

3.3 Additional Analyses of the Standard Measurements............................................... 6

3.4 Simulants with Known Anion Concentrations ........................................................... 6

3.5 Calibration Standards - Measured as Samples .......................................................... 7

3.6 Standards Prepared from Dried Salts........................................................................ 7

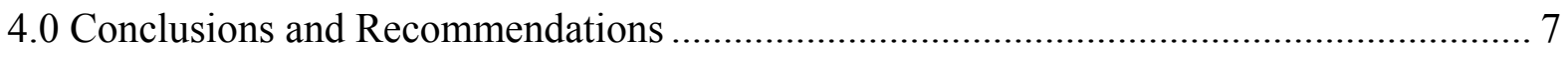

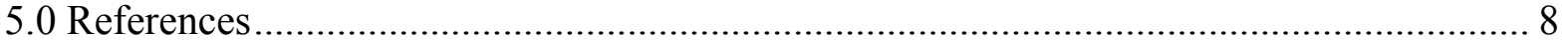




\section{LIST OF TABLES}

Table 1. Sequence of Analyses for a Typical Sludge Sample Set ...................................................... 3

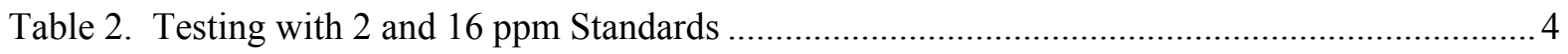

Table 3. Bounds on Potential Absolute Biases between the M-13 and M-14 ICS-3000 Instruments at

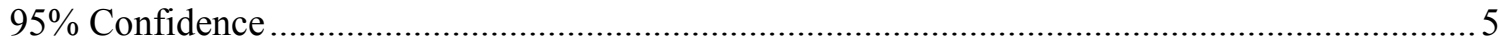

Table 4 95\% Confidence Intervals for the Anion Mean Concentration (ppm) by Instrument .............. 6 


\section{LIST OF ABBREVIATIONS}

$\begin{array}{ll}\text { ACES } & \begin{array}{l}\text { Applied Computational Engineering and Statistics } \\ \text { DWPF }\end{array} \\ \text { EG } & \text { Defense Waste Processing Facility } \\ \text { HLW } & \text { High Level Waste } \\ \text { IC } & \text { Ion Chromatography } \\ \text { JMP } & \text { Statistical software package from SAS Institute, Inc. [4] } \\ \text { NIST } & \text { National Institute of Standards and Technology } \\ \text { REDOX } & \text { Reduction-Oxidation } \\ \text { RFIC } & \text { Reagent Free Ion Chromatography } \\ \text { SME } & \text { Slurry Mix Evaporator } \\ \text { SRAT } & \text { Sludge Receipt and Adjustment Tank } \\ \text { SRNL } & \text { Savannah River National Laboratory } \\ \text { TTR } & \text { Technical Task Request } \\ \text { TT\&QA } & \text { Task Technical and Quality Assurance }\end{array}$




\subsection{INTRODUCTION}

The Defense Waste Processing Facility (DWPF) requires the analysis of specific anions at various stages of its processing of high level waste (HLW). The anions of interest to the DWPF are fluoride, formate, chloride, nitrite, nitrate, sulfate, oxalate, and phosphate. The anion analysis is used to evaluate process chemistry including formic acid / nitric acid additions to establish optimum conditions for mercury stripping, reduction-oxidation (REDOX) chemistry for the melter, nitrite destruction, etc. The DWPF Laboratory (Lab) has recently replaced the Dionex DX-500 ion chromatography (IC) systems that had been used since 1998 by the first of two new ICS-3000 systems. The replacement effort was necessary due to the vendor of the DX-500 systems no longer supporting service contracts after 2008 .

DWPF purchased three new ICS-3000 systems in September of 2006. The ICS-3000 instruments are (a) designed to be more stable using an eluent generator to make eluent, (b) require virtually no daily chemical handling by the analysts, (c) require less line breaks in the hood, and (d) generally require less maintenance due to the pump configuration only using water versus the current system where the pump uses various hydroxide concentrations. The ICS-3000 instruments also allow the DWPF to maintain current service contracts, which support routine preventive maintenance and emergency support for larger problems such as component failure.

One of the three new systems was set up in the DWPF Lab trailers in January of 2007 to be used for the development of methods and procedures. This system will continue to be used for training, new method development and potential improvements to current methods. The qualification of the other two ICS-3000 instruments was a phased effort. This effort was supported by the Applied Computational Engineering and Statistical (ACES) group of the Savannah River National Laboratory (SRNL) as authorized by the Technical Task Request (TTR) [1] and as directed by the corresponding Task Technical and Quality Assurance (TT\&QA) plan [2]. The installation of the first "rad" system into the M-13 Lab module required modifications to both the Lab module and to the radiohood. The installation was completed in July 2008. The testing of this system was conducted as directed by the TT\&QA plan [2], and the instrument was qualified for use at the DWPF Lab as documented in [3]. As part of that evaluation, a recommendation was made that the second ICS-3000 be installed in the M-14 module and that qualification testing of that system be conducted. The purpose of this technical report is to provide a review of the data generated by these tests that will lead to the recommendation for the qualification of the M-14 ICS-3000 instrument.

\subsection{Experimental Procedure}

As indicated above, the second "rad" ICS-3000 was implemented in the hood in M-14; it was debugged and tested to ensure that it was set up to the vendor's specifications and that it was performing as anticipated. The testing of the M-14 ICS-3000 versus the M-13 ICS-3000 instruments was a head-to-head comparison involving standards, process samples, and simulant samples. The units of measurement for the sample results generated by these two instruments are given in milligrams per kilogram of slurry or, equivalently, in parts per million (ppm). The units of measurement for the standard results generated by these two instruments are given in milligrams per liter $(\mathrm{mg} / \mathrm{L})$ or, equivalently, in parts per million (ppm).

In the subsections that follow, a comparison between the analytical methods of the two types of instruments is provided; the calibrations of the two instruments are discussed; and a description of the protocols utilized in measuring the simulant sample analyses, spiked sample analyses, and sludge sample analyses are provided. 


\subsection{Method Development}

The IC-3000 systems use a hydroxide based eluent and the AS-11 separator column. The ICS-3000 systems use what is called Reagent Free Ion Chromatography (RFIC). The RFIC is done with an Eluent Generator (EG) Module, which contains a cartridge with a quantitative amount of $4 \mathrm{M}$ potassium hydroxide $(\mathrm{KOH})$. The required eluent $(\mathrm{KOH})$ concentration is then made according to specifications prescribed in the method. The EG allows lower concentrations of hydroxide to be prepared more precisely and requires no mixing, which in turn allows for better separation than provided by the DX-500 systems of some of the more easily eluted smaller monovalent anions and provides more stable retention times.

\subsection{Calibration}

The ICS-3000 instruments were calibrated with NIST* traceable standards. Calibration solutions were prepared using 8 individual $1000 \mathrm{ppm}$ anion solutions. The calibration verification standards (check standards) are prepared with a NIST traceable multi-anion standard that includes 7 anions and nitrite. The nitrite solution is from a different vendor than the individual nitrite solution used for the calibration solutions.

The IC instruments are calibrated over a range of $1 \mathrm{ppm}$ to $20 \mathrm{ppm}$ for each anion, and the samples are diluted to ensure that the anion concentrations are in this range. DWPF routinely performs a $500 \mathrm{X}$ and a 5000X dilution. The dilutions are set up so that each anion's concentration will be within the 1 to $20 \mathrm{ppm}$ range of the calibration in one or both dilutions. The 500X dilution is used to provide "less than" values for anions not typically found in the sludge, i.e. fluoride, chloride and phosphate. The dilutions are also limited due to radiation levels in the samples for ALARA purposes. The IC instrument calibration is verified daily using a $2 \mathrm{ppm}$ standard and a $16 \mathrm{ppm}$ verification standard. Included as part of the data in Table A1 of Appendix A are measurements generated by the ICS-3000 instruments for such standards. Exhibit A1 of Appendix A provides a series of plots of these measurements. A statistical review of these data is provided in Section 3.

\subsection{Sludge Sample Analysis}

Another crosscheck of the two instrument conducted as part of this study is the direct comparison between the M-13 primary ICS-3000 used in sample analysis and the M-14 ICS-3000 with actual SRAT Receipt, SRAT Product and SME Product material. Six samples were analyzed, with 2 samples coming from each of the major tanks. As mentioned earlier, the sample preparation process involves preparing two dilutions, a 500X and a 5000X. Typically 4 Pnut vials of sludge are used. From each Pnut vial a roughly $1 \mathrm{~g}$ of sample to $100 \mathrm{~g}$ dilution with deionized water is made. From each of the four $100 \mathrm{X}$ dilutions, $500 \mathrm{X}$ and $5000 \mathrm{X}$ dilutions are prepared. Approximately $20 \mathrm{~mL}$ of each of the 8 dilutions is removed from the DWPF shielded cells to a glovebox where the final sample is filtered into an IC autosampler vial. A typical sample run on the IC is provided in Table 1.

* NIST - National Institute of Standards and Technology 
SRNL-STI-2009-00686

Revision 0

Table 1. Sequence of Analyses for a Typical Sludge Sample Set

\begin{tabular}{|c|}
\hline Blank \\
\hline 2 ppm check standard \\
\hline 16 ppm check standard \\
\hline 5000X dilution 1 \\
\hline 5000X dilution 2 \\
\hline 5000X dilution 3 \\
\hline 5000X dilution 4 \\
\hline Spiked Sample \\
\hline 500X dilution 1 \\
\hline 500X dilution 2 \\
\hline 500X dilution 3 \\
\hline 500X dilution 4 \\
\hline 2 ppm check standard \\
\hline 16 ppm check standard \\
\hline
\end{tabular}

The measurements resulting from these comparisons are included in Table A1 in Appendix A. Exhibit A2 in Appendix A provides a series of plots of these measurements. In this exhibit, the differences of the raw measurements from the two instruments are plotted for those anions whose concentrations in the process samples are typically above detection limits. A statistical review of these data is provided in Section 3.

\subsection{Simulated Sample Analysis}

Simulated samples, for the crosscheck work, were prepared from NIST traceable individual $1000 \mathrm{ppm}$ anion solutions. The simulated samples were prepared to match the anion concentrations for the average SRAT Receipt, SRAT Product, and SME samples in both the 500X and 5000X dilution. Anions typically not found in the sludge or that are diluted below detection, were added at the lowest calibration level, $1 \mathrm{ppm}$, to not only ensure that these anions could be measured at this concentration but to ensure that "tailing" from a larger peak would not prevent accurate measurement of these anions. In the 500X dilutions there are anion concentrations outside the $1-20 \mathrm{ppm}$ calibration range. These anions would be quantified on the 5000X dilution; however, they would still be in the $500 \mathrm{X}$ dilution and could affect other peaks in the chromatograph. Measurements generated by this crosscheck work are provided as part of the data in Table A1 in Appendix A. Exhibit A3 in Appendix A provides plots of these results grouped by type of simulant and the known concentrations of the anions. A statistical review of these data is provided in Section 3.

\subsection{Analysis of Calibration Standards as Samples}

Measurements were conducted on the 1,10 , and $20 \mathrm{ppm}$ standards that are used for calibrating the instruments. The measurements generated by this crosscheck work are provided as part of the data in Table A2 in Appendix A. Exhibit A4 in Appendix A provides plots of these results. A statistical review of these data is provided in Section 3.

\subsection{Analysis of Standards Prepared from Dried Salts}

DWPF Lab prepared 10 ppm solutions for each individual anion from dried salts and measured these solutions with both IC instruments. These measurements provide an independent verification of the calibration curves of the instruments as well as verifying the ability of the instruments to provide positive identification of individual anions. The measurements generated by this crosscheck work are provided as part of the data in Table A2 in Appendix A. Exhibit A5 in Appendix A provides a plot of these results. A statistical review of these data is provided in Section 3. 


\subsection{Statistical ANAlysis}

In this section, the statistical comparisons of the measurements generated by the M-13 and M-14 ICS3000 instruments from the crosscheck work described above are conducted and evaluated. JMP Version 7.0.2 [4] was used to perform these analyses. These statistical results served as the basis for the qualification of the M-14 ICS-3000 instrument, and the statistical comparisons of the following sections provide the justification for the qualification.

\subsection{Preliminary Comparisons Using Standards}

The initial crosschecking of the instruments included the measurement of 2 and 16 ppm standards. As indicated above, the data from this work are provided in Table A1. Table 2 below provides the minimum (min) and maximum (max) values for each anion for each instrument. The sample results consistently met the pre-existing criteria of $20 \%$ agreement limit for the $2 \mathrm{ppm}$ standard (i.e., 1.6 to $2.4 \mathrm{ppm}$ ) and $10 \%$ agreement limit for the $16 \mathrm{ppm}$ standard (i.e., 14.4 to $17.6 \mathrm{ppm}$ ) between the measurements and the standard values for each of the instruments. The results from these standards indicate that these instruments are capable of providing measurements for these standards that consistently meet the criteria for agreement with the reference values.

Table 2. Testing with 2 and 16 ppm Standards

\begin{tabular}{|c|c|c|c|c|c|}
\hline Standard & Instrument & Anion & $\begin{array}{c}\text { Number of } \\
\text { Observations }\end{array}$ & $\begin{array}{c}\text { Minimum } \\
\text { Measurement }(\mathrm{ppm})\end{array}$ & $\begin{array}{c}\text { Maximum } \\
\text { Measurement (ppm) }\end{array}$ \\
\hline $2 \mathrm{ppm}$ & M-13 & Chloride & 12 & 1.95 & 2.01 \\
\hline $2 \mathrm{ppm}$ & M-13 & Fluoride & 12 & 1.98 & 2.04 \\
\hline $2 \mathrm{ppm}$ & M-13 & Formate & 12 & 1.97 & 2.04 \\
\hline $2 \mathrm{ppm}$ & M-13 & Nitrate & 12 & 1.91 & 2.03 \\
\hline $2 \mathrm{ppm}$ & M-13 & Nitrite & 12 & 1.97 & 2.05 \\
\hline $2 \mathrm{ppm}$ & M-13 & Oxalate & 12 & 2.08 & 2.17 \\
\hline $2 \mathrm{ppm}$ & M-13 & Phosphate & 12 & 1.92 & 2.21 \\
\hline $2 \mathrm{ppm}$ & M-13 & Sulfate & 12 & 1.97 & 2.05 \\
\hline $2 \mathrm{ppm}$ & M-14 & Chloride & 12 & 1.89 & 1.94 \\
\hline $2 \mathrm{ppm}$ & M-14 & Fluoride & 12 & 1.99 & 2.05 \\
\hline $2 \mathrm{ppm}$ & M-14 & Formate & 12 & 1.97 & 2.07 \\
\hline $2 \mathrm{ppm}$ & M-14 & Nitrate & 12 & 1.83 & 2.01 \\
\hline $2 \mathrm{ppm}$ & M-14 & Nitrite & 12 & 1.95 & 2.07 \\
\hline $2 \mathrm{ppm}$ & M-14 & Oxalate & 12 & 2.06 & 2.12 \\
\hline $2 \mathrm{ppm}$ & M-14 & Phosphate & 12 & 1.86 & 2.02 \\
\hline $2 \mathrm{ppm}$ & M-14 & Sulfate & 12 & 1.91 & 1.97 \\
\hline $16 \mathrm{ppm}$ & M-13 & Chloride & 12 & 15.94 & 16.3 \\
\hline $16 \mathrm{ppm}$ & M-13 & Fluoride & 12 & 15.88 & 16.32 \\
\hline $16 \mathrm{ppm}$ & M-13 & Formate & 12 & 15.93 & 16.3 \\
\hline $16 \mathrm{ppm}$ & M-13 & Nitrate & 12 & 16.12 & 16.57 \\
\hline $16 \mathrm{ppm}$ & M-13 & Nitrite & 12 & 15.56 & 16.53 \\
\hline $16 \mathrm{ppm}$ & M-13 & Oxalate & 12 & 16.01 & 16.33 \\
\hline $16 \mathrm{ppm}$ & M-13 & Phosphate & 12 & 15.66 & 16.13 \\
\hline $16 \mathrm{ppm}$ & M-13 & Sulfate & 12 & 16.03 & 16.47 \\
\hline $16 \mathrm{ppm}$ & M-14 & Chloride & 12 & 16.09 & 16.32 \\
\hline $16 \mathrm{ppm}$ & M-14 & Fluoride & 12 & 16.09 & 16.36 \\
\hline $16 \mathrm{ppm}$ & M-14 & Formate & 12 & 16.04 & 16.41 \\
\hline $16 \mathrm{ppm}$ & M-14 & Nitrate & 12 & 16.15 & 16.59 \\
\hline $16 \mathrm{ppm}$ & M-14 & Nitrite & 12 & 15.9 & 16.52 \\
\hline $16 \mathrm{ppm}$ & M-14 & Oxalate & 12 & 16.14 & 16.5 \\
\hline $16 \mathrm{ppm}$ & M-14 & Phosphate & 12 & 15.43 & 15.74 \\
\hline $16 \mathrm{ppm}$ & M-14 & Sulfate & 12 & 16 & 16.28 \\
\hline
\end{tabular}




\subsection{Full Set of Crosscheck Sample Results}

The measurements generated by the crosscheck testing (i.e., Table A1) for the process samples along with standards that were included in the analytical protocols were imported into a JMP spreadsheet for analysis. A comparison of the precision of the two instruments is investigated first. Exhibits A6a-A6h provide statistical comparisons of the raw measurements by each instrument of the $2 \mathrm{ppm}$ standards. In these exhibits, Levene's test was used to identify a statistically significant difference in the variability of the measurements from the two instruments. ${ }^{f}$ Based upon the results of this test, there is no indication of a statistically significant difference between the variances of the measurements for any of the anions for the two instruments for the 2 ppm standard. Exhibits A7aA7h provide statistical comparisons of the raw measurements by each instrument of the $16 \mathrm{ppm}$ standards. Based upon the results of this test, there is no indication of a statistically significant difference between the variances of the measurements for any of the anions for the two instruments for the $16 \mathrm{ppm}$ standard. Thus, these analyses indicate comparable precision for the two ICS-3000 instruments in the measurement of these standards.

A comparison of the relative bias between the two instruments is investigated next using the raw score differences (M-13 minus $\mathrm{M}-14$ ) in ppm. A relative bias between the two instruments is indicated at the $5 \%$ significance level if zero is not within the $95 \%$ confidence interval presented as part of each exhibit. The larger, in absolute value, of the upper and lower limits for these confidence limits is a conservative bound at $95 \%$ confidence for the bias between the two instruments for the type of measurements represented in the exhibits. Exhibits A8a-A8h provide a look at all of the data for each anion in turn, with other exhibits (A8i through A8bt) providing a comparison for the measurements from the two instruments grouped by type of sample. From the analyses of all of the data, at the 5\% significance level, the M-13 is biased high as compared to the M-14 for chloride, nitrate, phosphate, and sulfate, and the M-14 is biased high as compared to the M-13 for fluoride. Note that all of the available measurements for chloride, fluoride, and phosphate are from standards (compare Exhibits A8i, A8j, and A8s to Exhibits A8a, A8b, and A8g). For each of the other anions, various groupings of the measurements from the two instruments are compared. Table 3 provides the largest bounding (absolute) bias seen between the raw measurements from the two instruments over all of the exhibits from A8a through A8bt for each of the anions. The averages of the corresponding raw measurement of the M-13 instrument are also provided in this table along the biases expressed as percentages of these averages. The largest absolute bound for bias is $0.429 \mathrm{ppm}$ for phosphate, which, when expressed as a relative bias to the average of the M-13 measurements $(8.725 \mathrm{ppm})$, represents a bias of $4.9 \%$. These results indicate no issues with the performance of the M-14 instrument.

Table 3. Bounds on Potential Absolute Biases between the M-13 and M-14 ICS-3000 Instruments at $95 \%$ Confidence

\begin{tabular}{|c|c|c|c|c|c|c|c|c|}
\hline Anion & Chloride & Fluoride & Formate & Nitrate & Nitrite & Oxalate & Phosphate & Sulfate \\
\hline Bias (ppm) & 0.134 & 0.196 & 0.472 & 0.258 & 0.252 & 0.138 & 0.429 & 0.230 \\
\hline $\begin{array}{c}\text { M-13 Average } \\
\text { (ppm) }\end{array}$ & 8.695 & 8.698 & 12.550 & 9.575 & 14.643 & 8.885 & 8.725 & 8.810 \\
\hline $\begin{array}{c}\% \text { Bias } \\
\text { Relative to } \\
\text { M-13 Average }\end{array}$ & $1.5 \%$ & $2.3 \%$ & $3.8 \%$ & $2.7 \%$ & $1.7 \%$ & $1.6 \%$ & $4.9 \%$ & $2.6 \%$ \\
\hline
\end{tabular}

\footnotetext{
${ }^{f}$ The results from other statistical tests for equality of variance are also provided by JMP in these exhibits, but the conclusions made in this analysis utilized the results from Levene's test.
} 


\subsection{Additional Analyses of the Standard Measurements}

The measurements of the standards (2 ppm and $16 \mathrm{ppm}$ ) from Table A1 are revisited in this section to assess absolute bias for each of the ICS-3000 instruments. Exhibits A9a-A9p provide histograms and descriptive statistics for the raw score measurement data (in ppm) generated by the M-13 and M-14 ICS-3000 instruments for the 2- and 16-ppm standards. Each exhibit has the M-13 results followed by the $\mathrm{M}-14$ results in column format.

The minimum and maximum measurements for each anion by each instrument are provided in these exhibits, and these values were summarized in Table 2. The exhibits also include a $95 \%$ confidence interval for the mean anion concentration for each instrument. An underlying assumption in the development of these confidence intervals is that the Central Limit Theorem applies for the data in each of these exhibits, and this appears to be a reasonable assumption for almost all of these data. For a given standard, if an instrument's confidence interval for an anion contains the nominal concentration (i.e., 2 or $16 \mathrm{ppm}$ ) for that anion, then that instrument is unbiased for that anion for that standard. Table 4 summarizes the confidence interval information. The biased results are shown in red in this table. The two instruments reveal biases that follow similar patterns for these standards, and the sizes of the biases for the two instruments seen in these data are comparable. The largest bias for the $2 \mathrm{ppm}$ standards seen in this table is that for the M-13's oxalate data (2.15 ppm), which on a percentage basis is $7.5 \%$; the largest bias for the $16 \mathrm{ppm}$ standards seen in this table is that for the M13 's nitrate data $(16.47 \mathrm{ppm})$, which on a percentage basis is only $2.9 \%$. These results indicate that the biases seen in the M-14's measurement of these standards are of little practical concern in regards to its ability to provide reliable measurements for DWPF operations.

Table 4 95\% Confidence Intervals for the Anion Mean Concentration (ppm) by Instrument

\begin{tabular}{|c|c|c|c|c|c|c|c|c|}
\hline \multicolumn{9}{|c|}{ Results from the 2 ppm Standards } \\
\hline Instrument & Chloride & Fluoride & Formate & Nitrate & Nitrite & Oxalate & Phosphate & Sulfate \\
\hline M-13 & $\mathbf{1 . 9 7 - 1 . 9 9}$ & $\mathbf{2 . 0 0 - 2 . 0 3}$ & $1.99-2.02$ & $\mathbf{1 . 9 5 - 2 . 0 0}$ & $2.00-2.04$ & $\mathbf{2 . 1 2 - 2 . 1 5}$ & $1.98-2.09$ & $1.99-2.03$ \\
\hline M-14 & $\mathbf{1 . 9 0 - 1 . 9 3}$ & $\mathbf{2 . 0 2 - 2 . 0 4}$ & $2.00-2.04$ & $\mathbf{1 . 8 8 - 1 . 9 4}$ & $1.99-2.04$ & $\mathbf{2 . 0 8}-2.11$ & $\mathbf{1 . 9 2 - 1 . 9 9}$ & $\mathbf{1 . 9 4 - 1 . 9 6}$ \\
\hline \multicolumn{8}{|c|}{ Results from the 16 ppm Standards } \\
\hline Instrument & Chloride & Fluoride & Formate & Nitrate & Nitrite & Oxalate & Phosphate & Sulfate \\
\hline M-13 & $\mathbf{1 6 . 0 9 - 1 6 . 2 4 ~}$ & $\mathbf{1 6 . 0 5 - 1 6 . 2 2}$ & $\mathbf{1 6 . 0 6 - 1 6 . 2 2}$ & $\mathbf{1 6 . 2 8 - 1 6 . 4 7}$ & $16.00-16.36$ & $\mathbf{1 6 . 1 4 - 1 6 . 2 6}$ & $15.83-16.03$ & $\mathbf{1 6 . 1 6 - 1 6 . 3 5}$ \\
\hline M-14 & $\mathbf{1 6 . 1 4 - 1 6 . 2 3}$ & $\mathbf{1 6 . 1 6 - 1 6 . 2 9}$ & $\mathbf{1 6 . 1 6 - 1 6 . 3 2}$ & $\mathbf{1 6 . 2 6 - 1 6 . 4 5}$ & $\mathbf{1 6 . 0 6 - 1 6 . 2 9}$ & $\mathbf{1 6 . 2 8 - 1 6 . 3 9}$ & $\mathbf{1 5 . 5 7 - 1 5 . 6 7}$ & $\mathbf{1 6 . 0 3 - 1 6 . 1 7}$ \\
\hline
\end{tabular}

(Statistically significant biases at $\alpha=5 \%$ are listed in red)

\subsection{Simulants with Known Anion Concentrations}

Measurements of the simulants with known anion concentrations are presented in Exhibits A10a through A10ae. Each exhibit has the M-13 results followed by the M-14 results in column format. The type of simulant and known anion concentration are presented as part of the header information in each of the exhibits. An investigation into the potential bias for each set of measurements is presented in the form of a $95 \%$ confidence interval for the mean of the measurements. If the known value for the simulant falls within the confidence interval, then there is no indication, at the $5 \%$ level, of a statistically significant bias. Based upon the results presented in this exhibit, there are some statistically significant biases in these measurements. For the simulants with 1 ppm known concentrations, the largest $95 \%$ confidence bound for the absolute value of the bias is that for the M13 's oxalate measurements, $1.110 \mathrm{ppm}$ or $11 \%$, corresponding to the measurements of the $5000 \mathrm{X}$ dilution of the SME simulant. For the simulants at a known concentration of $1.5 \mathrm{ppm}$, the largest 
$95 \%$ confidence bound for the absolute value of the bias is that for the M-14's nitrate measurements, $1.371 \mathrm{ppm}$ or $8.6 \%$, corresponding to the measurements of the $500 \mathrm{X}$ dilution of the SME simulant. For the simulants at a known concentration of $2.5 \mathrm{ppm}$, the largest $95 \%$ confidence bound for the absolute value of the bias is that for the M-13's phosphate measurements, $2.731 \mathrm{ppm}$ or $9.2 \%$, corresponding to the measurements of the $500 \mathrm{X}$ dilution of the SME simulant. For these simulants with known concentrations of $6 \mathrm{ppm}$ or larger, the largest 95\% confidence bound for the absolute value of the bias is that for the M-13's formate measurements, $53.71 \mathrm{ppm}$ or $10.6 \%$, corresponding to the measurements of the $500 \mathrm{X}$ dilution of the SME simulant with a reference value of $60 \mathrm{ppm}$ for formate. Since the calibrations of the instruments are only to $20 \mathrm{ppm}$, the bias confidence limit of $10.6 \%$ for $60 \mathrm{ppm}$ concentrations is indicative of a reasonably linear response curve beyond the calibration interval. The results from these investigations indicate that the biases seen in the M-14's measurement of these simulants are of little practical concern in regards to its ability to provide reliable measurements for DWPF operations.

\subsection{Calibration Standards - Measured as Samples}

Linear regressions of the measured values versus the reference values for each anion were conducted for the measurements generated by processing the calibration standards as samples (i.e., the data shown in Exhibits 4a through 4c). These results are provided for each anion for each instrument in Exhibits A11a through A11p. The coefficient of determination, $\mathrm{R}^{2}$, value is provided for each of the regressions. This value represents the fraction of the variation of the measurements that is explained by the regression equation. The closer this value is to 1 the better. The $\mathrm{R}^{2}$ values shown in these exhibits are all $0.999^{+}$. Also shown in these exhibits are $95 \%$ confidence intervals for the intercept and slope of each of the fitted equations. Ideally, 0 should be in or near the confidence interval for each y-intercept, and 1 should be in or near the confidence interval for each slope. This is the case for each of these regressions.

A "Lack of Fit" test for the adequacy of a linear model to fit the measurement data for each of the regressions is also provided. That is, for each anion and for each instrument, the test addresses the question: Is there an indication of a need for a quadratic model instead of a simple, linear model to explain the relationship between the reference and measured values? A statistically significant, at the $5 \%$ level, lack of fit is indicated in the "Lack of Fit" tabulated output by a "Prob $>$ F" value of 0.05 or smaller. Over all of these results, only the phosphate and sulfate results for the M-13 instrument show any statistically significant lack of fit. For both phosphate and sulfate, the linear fit slightly under predicts the measured values (i.e., the measured values are slightly higher than the values of the fitted equation). These results raise no issues of practical concern, and certainly provide no indication of an issue with the performance of the M-14 instrument.

\subsection{Standards Prepared from Dried Salts}

Exhibits A12a and A12b provide statistical analyses of the limited data generated by the measurement of the standards prepared from dried salts. These are the data shown in Exhibit A5. For the M-13, all of the measurements fall between 9.63 and $10.15 \mathrm{ppm}$, and for the M-14, all of the measurements fall between 9.54 and $10.58 \mathrm{ppm}$. For each of the two instruments, there are statistically significant, at the 5\% level, differences among the measurement means for the anions of interest. These differences are not seen as raising any practical concern regarding the performance of the M-14 instrument.

\subsection{CONCLUSIONS AND RECOMMENDATIONS}

The test results of the evaluation of the ICS-3000 in M-14 indicate that the performance of this new instrument is comparable to the performance of the M-13 instrument. Thus, the ICS-3000 system 
installed in 221-S M-14 is recommended for use at the DWPF Lab and is, thus, deemed qualified for such use. The qualification testing was a head-to-head comparison of the M-14 ICS-3000 instrument with the M-13 ICS-3000 system. The crosscheck work included standards for instrument calibration and calibration verifications and standards for individual anion analysis, which were NIST traceable. In addition the crosscheck work included the preparation of simulated SRAT Receipt, SRAT Product, and SME samples, along with actual Sludge Batch 5 material from the SRAT and SME tanks.

\subsection{REFERENCES}

[1] Mahannah, RN, "Technical Task Request: Perform Data Evaluation from the New ICS-3000 Ion Chromatography Systems versus the Current DX-500 IC," HLW/DWPF/TTR-2007-0023, Revision 0, August 2, 2007.

[2] Edwards, TB, "Task Technical \& QA Plan: Perform Data Evaluation of the New ICS-300 Ion Chromatography Systems versus the Current DX-500 Systems,” WSRC-STI-2007000449, Revision 0, August 27, 2007.

[3] Mahannah, RN and TB Edwards, "Qualification of the First ICS-3000 Ion Chromatograph for Use at the Defense Waste Processing Facility," SRNL-STI-2009-00323, Revision 0, June 2009.

[4] JMP Version 7.0.2, SAS Institute, Inc., Cary NC, 1989-2007. 


\section{Appendix A. Tables and Exhibits}


Table A1. Measurements of Standards and SRAT Receipt (SR), SRAT Product (SP), and SME Samples

\begin{tabular}{|c|c|c|c|c|c|c|c|c|c|c|c|}
\hline Date & $\begin{array}{c}\text { Nominal } \\
\text { DF }\end{array}$ & Tank & $\begin{array}{c}\text { Sample } \\
\text { Number }\end{array}$ & $\begin{array}{l}\text { Type of } \\
\text { Sample }\end{array}$ & $\begin{array}{c}\text { Bottle } \\
\text { ID }\end{array}$ & Anion & DF & $\begin{array}{c}\text { M-13 ICS3000 } \\
\text { raw (ppm) }\end{array}$ & $\begin{array}{c}\text { M-14 ICS3000 } \\
\text { raw (ppm) }\end{array}$ & $\begin{array}{l}\text { M-13 ICS3000 } \\
\text { FINAL (ppm) } \\
\end{array}$ & $\begin{array}{l}\text { M-14 ICS3000 } \\
\text { FINAL (ppm) } \\
\end{array}$ \\
\hline $09 / 22 / 2009$ & 1 & SR & 16367 & standard & $2 \mathrm{ppm}$ & Fluoride & 1 & 2.04 & 2.05 & 2.04 & 2.05 \\
\hline $09 / 22 / 2009$ & 1 & SR & 16367 & standard & $2 \mathrm{ppm}$ & Formate & 1 & 2.01 & 2.01 & 2.01 & 2.01 \\
\hline $09 / 22 / 2009$ & 1 & SR & 16367 & standard & $2 \mathrm{ppm}$ & Chloride & 1 & 2 & 1.93 & 2 & 1.93 \\
\hline $09 / 22 / 2009$ & 1 & SR & 16367 & standard & $2 \mathrm{ppm}$ & Nitrite & 1 & 2.04 & 1.99 & 2.04 & 1.99 \\
\hline $09 / 22 / 2009$ & 1 & SR & 16367 & standard & $2 \mathrm{ppm}$ & Nitrate & 1 & 2.03 & 1.93 & 2.03 & 1.93 \\
\hline $09 / 22 / 2009$ & 1 & SR & 16367 & standard & $2 \mathrm{ppm}$ & Sulfate & 1 & 2.05 & 1.97 & 2.05 & 1.97 \\
\hline $09 / 22 / 2009$ & 1 & SR & 16367 & standard & $2 \mathrm{ppm}$ & Oxalate & 1 & 2.17 & 2.11 & 2.17 & 2.11 \\
\hline $09 / 22 / 2009$ & 1 & SR & 16367 & standard & $2 \mathrm{ppm}$ & Phosphate & 1 & 2.07 & 1.99 & 2.07 & 1.99 \\
\hline $09 / 22 / 2009$ & 1 & SR & 16367 & standard & $16 \mathrm{ppm}$ & $\begin{array}{l}\text { Fluoride } \\
\end{array}$ & 1 & 16.24 & 16.34 & 16.24 & 16.34 \\
\hline $09 / 22 / 2009$ & 1 & SR & 16367 & standard & $16 \mathrm{ppm}$ & Formate & 1 & 16 & 16.1 & 16 & 16.1 \\
\hline $09 / 22 / 2009$ & 1 & SR & 16367 & standard & $16 \mathrm{ppm}$ & Chloride & 1 & 16.29 & 16.25 & 16.29 & 16.25 \\
\hline $09 / 22 / 2009$ & 1 & SR & 16367 & standard & $16 \mathrm{ppm}$ & Nitrite & 1 & 16.17 & 16.06 & 16.17 & 16.06 \\
\hline $09 / 22 / 2009$ & 1 & SR & 16367 & standard & $16 \mathrm{ppm}$ & Nitrate & 1 & 16.56 & 16.48 & 16.56 & 16.48 \\
\hline $09 / 22 / 2009$ & 1 & SR & 16367 & standard & $16 \mathrm{ppm}$ & Sulfate & 1 & 16.44 & 16.24 & 16.44 & 16.24 \\
\hline $09 / 22 / 2009$ & 1 & SR & 16367 & standard & $16 \mathrm{ppm}$ & Oxalate & 1 & 16.3 & 16.3 & 16.3 & 16.3 \\
\hline $09 / 22 / 2009$ & 1 & SR & 16367 & standard & $16 \mathrm{ppm}$ & Phosphate & 1 & 16.13 & 15.63 & 16.13 & 15.63 \\
\hline $09 / 22 / 2009$ & 5000 & SR & 16367 & process & 5893 & $\begin{array}{l}\text { Fluoride } \\
\end{array}$ & 5107.72157 &. &. &. &. \\
\hline $09 / 22 / 2009$ & 5000 & SR & 16367 & process & 5893 & Formate & 5107.72157 & . & . & . & . \\
\hline $09 / 22 / 2009$ & 5000 & SR & 16367 & process & 5893 & Chloride & 5107.72157 & . & . & 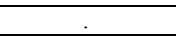 & . \\
\hline $09 / 22 / 2009$ & 5000 & SR & 16367 & process & 5893 & Nitrite & 5107.72157 & . & . & . & . \\
\hline $09 / 22 / 2009$ & 5000 & SR & 16367 & process & 5893 & Nitrate & 5107.72157 & . & . & . & . \\
\hline $09 / 22 / 2009$ & 5000 & SR & 16367 & process & 5893 & Sulfate & 5107.72157 & . & . & . & . \\
\hline $09 / 22 / 2009$ & 5000 & SR & 16367 & process & 5893 & Oxalate & 5107.72157 &. & - & &. \\
\hline $09 / 22 / 2009$ & 5000 & SR & 16367 & process & 5893 & Phosphate & 5107.72157 &. &. &. &. \\
\hline $09 / 22 / 2009$ & 5000 & SR & 16367 & process & 5895 & $\begin{array}{l}\text { Fluoride } \\
\end{array}$ & 5122.99578 &. & -1 & - & . \\
\hline $09 / 22 / 2009$ & 5000 & SR & 16367 & process & 5895 & Formate & 5122.99578 &. & . &. &. \\
\hline $09 / 22 / 2009$ & 5000 & SR & 16367 & process & 5895 & Chloride & 5122.99578 & . & . & . & . \\
\hline $09 / 22 / 2009$ & 5000 & SR & 16367 & process & 5895 & Nitrite & 5122.99578 & . & . & . & . \\
\hline $09 / 22 / 2009$ & 5000 & SR & 16367 & process & 5895 & Nitrate & 5122.99578 & . & . & . & . \\
\hline $09 / 22 / 2009$ & 5000 & SR & 16367 & process & 5895 & Sulfate & 5122.99578 & . & . & . & . \\
\hline $09 / 22 / 2009$ & 5000 & SR & 16367 & process & 5895 & Oxalate & 5122.99578 & . & . & . & . \\
\hline $09 / 22 / 2009$ & 5000 & SR & 16367 & process & 5895 & Phosphate & 5122.99578 & . & . & . & . \\
\hline $09 / 22 / 2009$ & 5000 & SR & 16367 & process & 5896 & Fluoride & 5132.81459 & . & . & . & . \\
\hline $09 / 22 / 2009$ & 5000 & SR & 16367 & process & 5896 & Formate & 5132.81459 & . & . & . & . \\
\hline $09 / 22 / 2009$ & 5000 & SR & 16367 & process & 5896 & Chloride & 5132.81459 & . & . &. & . \\
\hline $09 / 22 / 2009$ & 5000 & SR & 16367 & process & 5896 & Nitrite & 5132.81459 &. & -1 &. &. \\
\hline $09 / 22 / 2009$ & 5000 & SR & 16367 & process & 5896 & Nitrate & 5132.81459 & . & . & . & . \\
\hline $09 / 22 / 2009$ & 5000 & SR & 16367 & process & 5896 & Sulfate & 5132.81459 & . & . & . & . \\
\hline $09 / 22 / 2009$ & 5000 & SR & 16367 & process & 5896 & Oxalate & 5132.81459 &. & . &. & . \\
\hline $09 / 22 / 2009$ & 5000 & SR & 16367 & process & 5896 & Phosphate & 5132.81459 & . & . & . & . \\
\hline $09 / 22 / 2009$ & 5000 & SR & 16367 & process & 5897 & Fluoride & 5268.9964 & & & . & . \\
\hline $09 / 22 / 2009$ & 5000 & SR & 16367 & process & 5897 & Formate & 5268.9964 & 1.58 & 1.53 & . & . \\
\hline $09 / 22 / 2009$ & 5000 & SR & 16367 & process & 5897 & $\begin{array}{l}\text { Chloride } \\
\end{array}$ & 5268.9964 & & & . & . \\
\hline $09 / 22 / 2009$ & 5000 & SR & 16367 & process & 5897 & Nitrite & 5268.9964 & 1.67 & 1.7 & . & . \\
\hline $09 / 22 / 2009$ & 5000 & SR & 16367 & process & 5897 & Nitrate & 5268.9964 & 1.9 & 1.96 & . & . \\
\hline $09 / 22 / 2009$ & 5000 & SR & 16367 & process & 5897 & Sulfate & 5268.9964 & . & . & . & . \\
\hline $09 / 22 / 2009$ & 5000 & SR & 16367 & process & 5897 & Oxalate & 5268.9964 & . & . & . & . \\
\hline $09 / 22 / 2009$ & 5000 & SR & 16367 & process & 5897 & Phosphate & 5268.9964 & & & & \\
\hline $09 / 22 / 2009$ & 16 & SR & 16367 & standard & 9999 & $\begin{array}{l}\text { Fluoride } \\
\end{array}$ & 2 & 8.16 & 8.16 & 102.01 & 102.01 \\
\hline $09 / 22 / 2009$ & 16 & SR & 16367 & standard & 9999 & Formate & 2 & 8.99 & 8.95 & 102.52 & 102.37 \\
\hline $09 / 22 / 2009$ & 16 & SR & 16367 & standard & 9999 & Chloride & 2 & 8.17 & 8.05 & 102.08 & 100.66 \\
\hline
\end{tabular}


Table A1. Measurements of Standards and SRAT Receipt (SR), SRAT Product (SP), and SME Samples

\begin{tabular}{|c|c|c|c|c|c|c|c|c|c|c|c|}
\hline Date & $\begin{array}{c}\begin{array}{c}\text { Nominal } \\
\text { DF }\end{array} \\
\end{array}$ & Tank & $\begin{array}{c}\text { Sample } \\
\text { Number }\end{array}$ & $\begin{array}{l}\text { Type of } \\
\text { Sample }\end{array}$ & $\begin{array}{c}\text { Bottle } \\
\text { ID }\end{array}$ & Anion & DF & $\begin{array}{c}\text { M-13 ICS3000 } \\
\text { raw (ppm) }\end{array}$ & $\begin{array}{c}\text { M-14 ICS3000 } \\
\text { raw (ppm) }\end{array}$ & $\begin{array}{l}\text { M-13 ICS3000 } \\
\text { FINAL (ppm) } \\
\end{array}$ & $\begin{array}{l}\text { M-14 ICS3000 } \\
\text { FINAL (ppm) } \\
\end{array}$ \\
\hline $09 / 22 / 2009$ & 16 & SR & 16367 & standard & $\begin{array}{l}9999 \\
\end{array}$ & Nitrite & 2 & 8.88 & 8.8 & 100.58 & 99.33 \\
\hline $09 / 22 / 2009$ & 16 & SR & 16367 & standard & 9999 & Nitrate & 2 & 9.35 & 9 & 104.98 & 100.28 \\
\hline $09 / 22 / 2009$ & 16 & SR & 16367 & standard & 9999 & Sulfate & 2 & 8.37 & 8.15 & 104.59 & 101.87 \\
\hline $09 / 22 / 2009$ & 16 & SR & 16367 & standard & 9999 & Oxalate & 2 & 8.37 & 8.35 & 104.61 & 104.41 \\
\hline $09 / 22 / 2009$ & 16 & SR & 16367 & standard & 9999 & Phosphate & 2 & 8.04 & 7.71 & 100.48 & 96.43 \\
\hline $09 / 22 / 2009$ & 500 & SR & 16367 & process & 5886 & $\begin{array}{l}\text { Fluoride } \\
\end{array}$ & 508.335201 & & & & \\
\hline $09 / 22 / 2009$ & 500 & SR & 16367 & process & 58866 & Formate & 508.335201 & 16.09 & 15.81 & 8179 & 8037 \\
\hline $09 / 22 / 2009$ & 500 & SR & 16367 & process & 5886 & Chloride & 508.335201 & & & & \\
\hline $09 / 22 / 2009$ & 500 & SR & 16367 & process & 5886 & Nitrite & 508.335201 & 17.57 & 17.34 & 8931 & 8815 \\
\hline $09 / 22 / 2009$ & 500 & SR & 16367 & process & 5886 & Nitrate & 508.335201 & 19.93 & 19.68 & 10131 & 10004 \\
\hline $09 / 22 / 2009$ & 500 & SR & 16367 & process & 5886 & Sulfate & 508.335201 & 1.33 & 1.23 & 678 & 627 \\
\hline $09 / 22 / 2009$ & 500 & SR & 16367 & process & 5886 & Oxalate & 508.335201 & 2.88 & 2.82 & 1463 & 1431 \\
\hline $09 / 22 / 2009$ & 500 & SR & 16367 & process & 5886 & Phosphate & 508.335201 & & . & & \\
\hline $09 / 22 / 2009$ & 500 & SR & 16367 & process & 5887 & Fluoride & 493.263423 & & & & \\
\hline $09 / 22 / 2009$ & 500 & SR & 16367 & process & 5887 & Formate & 493.263423 & 16.61 & 15.78 & 8194 & 7784 \\
\hline $09 / 22 / 2009$ & 500 & SR & 16367 & process & 5887 & Chloride & 493.263423 & & & & \\
\hline $09 / 22 / 2009$ & 500 & SR & 16367 & process & 5887 & Nitrite & 493.263423 & 18.17 & 17.82 & 8963 & 8790 \\
\hline $09 / 22 / 2009$ & 500 & SR & 16367 & process & 5887 & Nitrate & 493.263423 & 20.59 & 20.46 & 10156 & 10092 \\
\hline $09 / 22 / 2009$ & 500 & SR & 16367 & process & 5887 & Sulfate & 493.263423 & 1.33 & 1.33 & 654 & 658 \\
\hline $09 / 22 / 2009$ & 500 & SR & 16367 & process & 5887 & Oxalate & 493.263423 & 2.96 & 2.92 & 1461 & 1441 \\
\hline $09 / 22 / 2009$ & 500 & SR & 16367 & process & 5887 & Phosphate & 493.263423 & . & . & . & . \\
\hline $09 / 22 / 2009$ & 500 & SR & 16367 & process & 5888 & Fluoride & 504.680603 & & & & \\
\hline $09 / 22 / 2009$ & 500 & SR & 16367 & process & 5888 & Formate & 504.680603 & 16.33 & 15.38 & 8241 & 7763 \\
\hline $09 / 22 / 2009$ & 500 & SR & 16367 & process & 5888 & Chloride & 504.680603 & & & & \\
\hline $09 / 22 / 2009$ & 500 & SR & 16367 & process & 5888 & Nitrite & 504.680603 & 17.8 & 17.38 & 8982 & 8771 \\
\hline $09 / 22 / 2009$ & 500 & SR & 16367 & process & 5888 & Nitrate & 504.680603 & 20.22 & 19.9 & 10205 & 10043 \\
\hline $09 / 22 / 2009$ & 500 & SR & 16367 & process & 5888 & Sulfate & 504.680603 & 1.29 & 1.24 & 650 & 627 \\
\hline $09 / 22 / 2009$ & 500 & SR & 16367 & process & 5888 & Oxalate & 504.680603 & 2.92 & 2.84 & 1472 & 1432 \\
\hline $09 / 22 / 2009$ & 500 & SR & 16367 & process & 5888 & Phosphate & 504.680603 & & . & & \\
\hline $09 / 22 / 2009$ & 500 & SR & 16367 & process & 5889 & $\begin{array}{l}\text { Fluoride } \\
\end{array}$ & 509.219796 & & & & \\
\hline $09 / 22 / 2009$ & 500 & SR & 16367 & process & 5889 & Formate & 509.219796 & 15.45 & 15.22 & 7869 & 7749 \\
\hline $09 / 22 / 2009$ & 500 & SR & 16367 & process & 5889 & Chloride & 509.219796 & & & & \\
\hline $09 / 22 / 2009$ & 500 & SR & 16367 & process & 5889 & Nitrite & 509.219796 & 17.55 & 17.38 & 8937 & 8850 \\
\hline $09 / 22 / 2009$ & 500 & SR & 16367 & process & 5889 & Nitrate & 509.219796 & 19.93 & 19.67 & 10149 & 10016 \\
\hline $09 / 22 / 2009$ & 500 & SR & 16367 & process & 5889 & Sulfate & 509.219796 & 1.29 & 1.25 & 657 & 635 \\
\hline $09 / 22 / 2009$ & 500 & SR & 16367 & process & 5889 & Oxalate & 509.219796 & 2.86 & 2.83 & 1456 & 1440 \\
\hline $09 / 22 / 2009$ & 500 & SR & 16367 & process & $\begin{array}{l}5889 \\
\end{array}$ & Phosphate & 509.219796 & & & & \\
\hline $09 / 22 / 2009$ & 500 & SR & 16367 & standard & $2 \mathrm{ppm}$ & Fluoride & 1 & 2.04 & 2.04 & 2.04 & 2.04 \\
\hline $09 / 22 / 2009$ & 500 & SR & 16367 & standard & $2 \mathrm{ppm}$ & Formate & 1 & 2 & 1.98 & 2 & 1.98 \\
\hline $09 / 22 / 2009$ & 500 & SR & 16367 & standard & $2 \mathrm{ppm}$ & Chloride & 1 & 2 & 1.94 & 2 & 1.94 \\
\hline $09 / 22 / 2009$ & 500 & SR & 16367 & standard & $2 \mathrm{ppm}$ & Nitrite & 1 & 2.04 & 2.07 & 2.04 & 2.07 \\
\hline $09 / 22 / 2009$ & 500 & SR & 16367 & standard & $2 \mathrm{ppm}$ & Nitrate & 1 & 2.01 & 2.01 & 2.01 & 2.01 \\
\hline $09 / 22 / 2009$ & 500 & SR & 16367 & standard & $2 \mathrm{ppm}$ & Sulfate & 1 & 2.01 & 1.96 & 2.01 & 1.96 \\
\hline $09 / 22 / 2009$ & 500 & SR & 16367 & standard & $2 \mathrm{ppm}$ & Oxalate & 1 & 2.13 & 2.11 & 2.13 & 2.11 \\
\hline $09 / 22 / 2009$ & 500 & SR & 16367 & standard & $2 \mathrm{ppm}$ & Phosphate & 1 & 1.94 & 1.96 & 1.94 & 1.96 \\
\hline $09 / 22 / 2009$ & 500 & SR & 16367 & standard & $16 \mathrm{ppm}$ & Fluoride & 1 & 16.25 & 16.36 & 16.25 & 16.36 \\
\hline $09 / 22 / 2009$ & 500 & SR & 16367 & standard & $16 \mathrm{ppm}$ & Formate & 1 & 16.03 & 16.24 & 16.03 & 16.24 \\
\hline $09 / 22 / 2009$ & 500 & SR & 16367 & standard & $16 \mathrm{ppm}$ & Chloride & 1 & 16.29 & 16.27 & 16.29 & 16.27 \\
\hline $09 / 22 / 2009$ & 500 & SR & 16367 & standard & $16 \mathrm{ppm}$ & Nitrite & 1 & 16.13 & 16.14 & 16.13 & 16.14 \\
\hline $09 / 22 / 2009$ & 500 & SR & 16367 & standard & $16 \mathrm{ppm}$ & Nitrate & 1 & 16.51 & 16.52 & 16.51 & 16.52 \\
\hline $09 / 22 / 2009$ & 500 & SR & 16367 & standard & $16 \mathrm{ppm}$ & Sulfate & 1 & 16.38 & 16.24 & 16.38 & 16.24 \\
\hline
\end{tabular}


Table A1. Measurements of Standards and SRAT Receipt (SR), SRAT Product (SP), and SME Samples

\begin{tabular}{|c|c|c|c|c|c|c|c|c|c|c|c|}
\hline Date & $\begin{array}{c}\begin{array}{c}\text { Nominal } \\
\text { DF }\end{array} \\
\end{array}$ & Tank & $\begin{array}{c}\text { Sample } \\
\text { Number }\end{array}$ & $\begin{array}{l}\text { Type of } \\
\text { Sample }\end{array}$ & $\begin{array}{c}\text { Bottle } \\
\text { ID }\end{array}$ & Anion & DF & $\begin{array}{c}\text { M-13 ICS3000 } \\
\text { raw (ppm) }\end{array}$ & $\begin{array}{c}\text { M-14 ICS3000 } \\
\text { raw (ppm) }\end{array}$ & $\begin{array}{l}\text { M-13 ICS3000 } \\
\text { FINAL (ppm) } \\
\end{array}$ & $\begin{array}{l}\text { M-14 ICS3000 } \\
\text { FINAL (ppm) } \\
\end{array}$ \\
\hline $09 / 22 / 2009$ & 500 & SR & 16367 & standard & $16 \mathrm{ppm}$ & Oxalate & 1 & 16.27 & 16.38 & 16.27 & 16.38 \\
\hline $09 / 22 / 2009$ & 500 & SR & 16367 & standard & $16 \mathrm{ppm}$ & Phosphate & 1 & 15.89 & 15.67 & 15.89 & 15.67 \\
\hline $09 / 29 / 2009$ & $\therefore$ & SR & 16507 & standard & $2 \mathrm{ppm}$ & Fluoride & 1 & 1.98 & 2.04 & 1.98 & 2.04 \\
\hline $09 / 29 / 2009$ & & SR & 16507 & standard & $2 \mathrm{ppm}$ & Formate & 1 & 2 & 2.03 & 2 & 2.03 \\
\hline $09 / 29 / 2009$ & . & SR & 16507 & standard & $2 \mathrm{ppm}$ & Chloride & 1 & 1.95 & 1.93 & 1.95 & 1.93 \\
\hline $09 / 29 / 2009$ & . & SR & 16507 & standard & $2 \mathrm{ppm}$ & Nitrite & 1 & 1.98 & 2.02 & 1.98 & 2.02 \\
\hline $09 / 29 / 2009$ & . & SR & 16507 & standard & $2 \mathrm{ppm}$ & Nitrate & 1 & 1.93 & 1.88 & 1.93 & 1.88 \\
\hline $09 / 29 / 2009$ & . & SR & 16507 & standard & $2 \mathrm{ppm}$ & Sulfate & 1 & 2 & 1.96 & 2 & 1.96 \\
\hline $09 / 29 / 2009$ &. & SR & 16507 & standard & $2 \mathrm{ppm}$ & Oxalate & 1 & 2.14 & 2.11 & 2.14 & 2.11 \\
\hline $09 / 29 / 2009$ & . & SR & 16507 & standard & $2 \mathrm{ppm}$ & Phosphate & 1 & 2.06 & 2.02 & 2.06 & 2.02 \\
\hline $09 / 29 / 2009$ & . & SR & 16507 & standard & $16 \mathrm{ppm}$ & $\begin{array}{l}\text { Fluoride } \\
\end{array}$ & 1 & 16.1 & 16.16 & 16.1 & 16.16 \\
\hline $09 / 29 / 2009$ & . & SR & 16507 & standard & $16 \mathrm{ppm}$ & Formate & 1 & 16.15 & 16.31 & 16.15 & 16.31 \\
\hline $09 / 29 / 2009$ & . & SR & 16507 & standard & $16 \mathrm{ppm}$ & Chloride & 1 & 16.13 & 16.15 & 16.13 & 16.15 \\
\hline $09 / 29 / 2009$ & . & SR & 16507 & standard & $16 \mathrm{ppm}$ & Nitrite & 1 & 16.15 & 15.92 & 16.15 & 15.92 \\
\hline $09 / 29 / 2009$ & . & SR & 16507 & standard & $16 \mathrm{ppm}$ & Nitrate & 1 & 16.32 & 16.36 & 16.32 & 16.36 \\
\hline $09 / 29 / 2009$ & . & SR & 16507 & standard & $16 \mathrm{ppm}$ & Sulfate & 1 & 16.21 & 16.01 & 16.21 & 16.01 \\
\hline $09 / 29 / 2009$ &. & SR & 16507 & standard & $16 \mathrm{ppm}$ & Oxalate & 1 & 16.21 & 16.36 & 16.21 & 16.36 \\
\hline $09 / 29 / 2009$ & 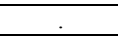 & SR & 16507 & standard & $16 \mathrm{ppm}$ & Phosphate & 1 & 16.09 & 15.65 & 16.09 & 15.65 \\
\hline $09 / 29 / 2009$ & 5000 & SR & 16507 & process & 6338 & Fluoride & 4976.90316 & & . & & 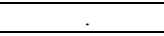 \\
\hline $09 / 29 / 2009$ & 5000 & SR & 16507 & process & 6338 & Formate & 4976.90316 &. &. &. & . \\
\hline $09 / 29 / 2009$ & 5000 & SR & 16507 & process & 6338 & Chloride & 4976.90316 & . & . & . & . \\
\hline $09 / 29 / 2009$ & 5000 & SR & 16507 & process & 6338 & Nitrite & 4976.90316 & . & . & . & . \\
\hline $09 / 29 / 2009$ & 5000 & SR & 16507 & process & 6338 & Nitrate & 4976.90316 & &. & & \\
\hline $09 / 29 / 2009$ & 5000 & SR & 16507 & process & 6338 & Sulfate & 4976.90316 & . &. & & . \\
\hline $09 / 29 / 2009$ & 5000 & SR & 16507 & process & 6338 & Oxalate & 4976.90316 & &. & & \\
\hline $09 / 29 / 2009$ & 5000 & SR & 16507 & process & 6338 & Phosphate & 4976.90316 &. &. & $\therefore$ & 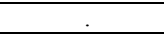 \\
\hline $09 / 29 / 2009$ & 5000 & SR & 16507 & process & 6339 & Fluoride & 4957.00335 & . & . & . & . \\
\hline $09 / 29 / 2009$ & 5000 & SR & 16507 & process & 6339 & Formate & 4957.00335 & . & . & . & . \\
\hline $09 / 29 / 2009$ & 5000 & SR & 16507 & process & 6339 & Chloride & 4957.00335 & . & . & - & . \\
\hline $09 / 29 / 2009$ & 5000 & SR & 16507 & process & 6339 & Nitrite & 4957.00335 & . & . & - & . \\
\hline $09 / 29 / 2009$ & 5000 & SR & 16507 & process & 6339 & Nitrate & 4957.00335 & . & . & . & . \\
\hline $09 / 29 / 2009$ & 5000 & SR & 16507 & process & 6339 & Sulfate & 4957.00335 & . & . &. & . \\
\hline $09 / 29 / 2009$ & 5000 & SR & 16507 & process & 6339 & Oxalate & 4957.00335 &. &. &. &. \\
\hline $09 / 29 / 2009$ & 5000 & SR & 16507 & process & 6339 & Phosphate & 4957.00335 & . &. & . & . \\
\hline $09 / 29 / 2009$ & 5000 & SR & 16507 & process & 6341 & $\begin{array}{l}\text { Fluoride } \\
\end{array}$ & 4973.69723 & . &. & . & - \\
\hline $09 / 29 / 2009$ & 5000 & SR & 16507 & process & 6341 & Formate & 4973.69723 & - &. & & - \\
\hline $09 / 29 / 2009$ & 5000 & SR & 16507 & process & 6341 & Chloride & 4973.69723 &. & . & & . \\
\hline $09 / 29 / 2009$ & 5000 & SR & 16507 & process & 6341 & Nitrite & 4973.69723 & 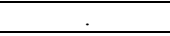 & . & & . \\
\hline $09 / 29 / 2009$ & 5000 & SR & 16507 & process & 6341 & Nitrate & 4973.69723 & 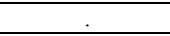 & . & 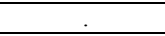 & . \\
\hline $09 / 29 / 2009$ & 5000 & SR & 16507 & process & 6341 & Sulfate & 4973.69723 & . & . & . & . \\
\hline $09 / 29 / 2009$ & 5000 & SR & 16507 & process & 6341 & Oxalate & 4973.69723 & . & . &. & . \\
\hline $09 / 29 / 2009$ & 5000 & SR & 16507 & process & 6341 & Phosphate & 4973.69723 & &. & $\therefore$ & $\dot{\square}$ \\
\hline $09 / 29 / 2009$ & 5000 & SR & 16507 & process & 6342 & Fluoride & 5100.93415 & & . & & - \\
\hline $09 / 29 / 2009$ & 5000 & SR & 16507 & process & 6342 & Formate & 5100.93415 & 1.45 & 1.45 &. & . \\
\hline $09 / 29 / 2009$ & 5000 & SR & 16507 & process & 6342 & Chloride & 5100.93415 & & & . & . \\
\hline $09 / 29 / 2009$ & 5000 & SR & 16507 & process & 6342 & Nitrite & 5100.93415 & 1.75 & 1.65 & . & . \\
\hline $09 / 29 / 2009$ & 5000 & SR & 16507 & process & 6342 & Nitrate & 5100.93415 & 1.88 & 1.78 & & \\
\hline $09 / 29 / 2009$ & 5000 & SR & 16507 & process & 6342 & Sulfate & 5100.93415 & & 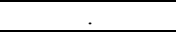 & & \\
\hline $09 / 29 / 2009$ & 5000 & SR & 16507 & process & 6342 & Oxalate & 5100.93415 & &. & &. \\
\hline $09 / 29 / 2009$ & 5000 & SR & 16507 & process & 6342 & Phosphate & 5100.93415 & & & & \\
\hline $09 / 29 / 2009$ & 16 & SR & 16507 & standard & 9999 & Fluoride & 2 & 8.1 & 8.16 & 101.25 & 102.05 \\
\hline
\end{tabular}


Table A1. Measurements of Standards and SRAT Receipt (SR), SRAT Product (SP), and SME Samples

\begin{tabular}{|c|c|c|c|c|c|c|c|c|c|c|c|}
\hline Date & $\begin{array}{c}\text { Nominal } \\
\text { DF }\end{array}$ & Tank & $\begin{array}{c}\text { Sample } \\
\text { Number }\end{array}$ & $\begin{array}{l}\text { Type of } \\
\text { Sample }\end{array}$ & $\begin{array}{c}\text { Bottle } \\
\text { ID }\end{array}$ & Anion & DF & $\begin{array}{l}\text { M-13 ICS3000 } \\
\text { raw (ppm) }\end{array}$ & $\begin{array}{l}\text { M-14 ICS3000 } \\
\text { raw (ppm) }\end{array}$ & $\begin{array}{l}\text { M-13 ICS3000 } \\
\text { FINAL (ppm) } \\
\end{array}$ & $\begin{array}{l}\text { M-14 ICS3000 } \\
\text { FINAL (ppm) } \\
\end{array}$ \\
\hline $09 / 29 / 2009$ & 16 & SR & 16507 & standard & $\begin{array}{l}9999 \\
\end{array}$ & Formate & 2 & 8.83 & 9.19 & 101.39 & 105.87 \\
\hline $09 / 29 / 2009$ & 16 & SR & 16507 & standard & 9999 & $\begin{array}{l}\text { Chloride } \\
\end{array}$ & 2 & 8.07 & 8.05 & 100.84 & 100.58 \\
\hline $09 / 29 / 2009$ & 16 & SR & 16507 & standard & 9999 & Nitrite & 2 & 8.99 & 9 & 101.42 & 102.22 \\
\hline $09 / 29 / 2009$ & 16 & SR & 16507 & standard & 9999 & Nitrate & 2 & 9.14 & 9.16 & 102.44 & 103.42 \\
\hline $09 / 29 / 2009$ & 16 & SR & 16507 & standard & 9999 & Sulfate & 2 & 8.2 & 8.07 & 102.5 & 100.94 \\
\hline $09 / 29 / 2009$ & 16 & SR & 16507 & standard & 9999 & Oxalate & 2 & 8.4 & 8.43 & 104.98 & 105.39 \\
\hline $09 / 29 / 2009$ & 16 & SR & 16507 & standard & 9999 & Phosphate & 2 & 8.1 & 7.76 & 101.25 & 97.05 \\
\hline $09 / 29 / 2009$ & 500 & SR & 16507 & process & 6313 & $\begin{array}{l}\text { Fluoride } \\
\end{array}$ & 488.348334 & & & & \\
\hline $\begin{array}{l}09 / 29 / 2009 \\
\end{array}$ & 500 & SR & 16507 & process & 6313 & Formate & 488.348334 & 14.84 & 14.93 & 7249 & 7290 \\
\hline $09 / 29 / 2009$ & 500 & SR & 16507 & process & 6313 & $\begin{array}{l}\text { Chloride } \\
\end{array}$ & 488.348334 & & & & \\
\hline $09 / 29 / 2009$ & 500 & SR & 16507 & process & 6313 & Nitrite & 488.348334 & 18.42 & 18.33 & 8995 & 8953 \\
\hline $09 / 29 / 2009$ & 500 & SR & 16507 & process & 6313 & Nitrate & 488.348334 & 19.38 & 19.23 & 9463 & 9393 \\
\hline $09 / 29 / 2009$ & 500 & SR & 16507 & process & 6313 & Sulfate & 488.348334 & 1.26 & 1.22 & 614 & 594 \\
\hline $09 / 29 / 2009$ & 500 & SR & 16507 & process & 6313 & Oxalate & 488.348334 & 2.48 & 2.42 & 1213 & 1182 \\
\hline $09 / 29 / 2009$ & 500 & SR & 16507 & process & 6313 & Phosphate & 488.348334 & & . & . & - \\
\hline $09 / 29 / 2009$ & 500 & SR & 16507 & process & 6318 & Fluoride & 487.330122 & & & & \\
\hline $09 / 29 / 2009$ & 500 & SR & 16507 & process & 6318 & Formate & 487.330122 & 14.65 & 14.75 & 7137 & 7189 \\
\hline $09 / 29 / 2009$ & 500 & SR & 16507 & process & 6318 & $\begin{array}{l}\text { Chloride } \\
\end{array}$ & 487.330122 & & & & \\
\hline $09 / 29 / 2009$ & 500 & SR & 16507 & process & 6318 & Nitrite & 487.330122 & 18.15 & 18.14 & 8847 & 8840 \\
\hline $09 / 29 / 2009$ & 500 & SR & 16507 & process & 6318 & Nitrate & 487.330122 & 19.1 & 18.99 & 9306 & 9257 \\
\hline $09 / 29 / 2009$ & 500 & SR & 16507 & process & 6318 & Sulfate & 487.330122 & 1.27 & 1.2 & 620 & 583 \\
\hline $09 / 29 / 2009$ & 500 & SR & 16507 & process & 6318 & Oxalate & 487.330122 & 2.47 & 2.39 & 1204 & 1164 \\
\hline $09 / 29 / 2009$ & 500 & SR & 16507 & process & 6318 & Phosphate & 487.330122 & & & & \\
\hline $09 / 29 / 2009$ & 500 & $\begin{array}{l}\text { SR } \\
\end{array}$ & 16507 & process & 6319 & $\begin{array}{l}\text { Fluoride } \\
\end{array}$ & 495.459741 & & & & \\
\hline $09 / 29 / 2009$ & 500 & SR & 16507 & process & 6319 & Formate & 495.459741 & 14.23 & 14.36 & 7050 & 7114 \\
\hline $09 / 29 / 2009$ & 500 & SR & 16507 & process & 6319 & $\begin{array}{l}\text { Chloride } \\
\end{array}$ & 495.459741 & & & & \\
\hline $09 / 29 / 2009$ & 500 & SR & 16507 & process & 6319 & Nitrite & 495.459741 & 17.65 & 17.57 & 8744 & 8705 \\
\hline $09 / 29 / 2009$ & 500 & SR & 16507 & process & 6319 & Nitrate & 495.459741 & 18.53 & 18.44 & 9179 & 9136 \\
\hline $09 / 29 / 2009$ & 500 & SR & 16507 & process & 6319 & Sulfate & 495.459741 & 1.2 & 1.16 & 596 & 573 \\
\hline $09 / 29 / 2009$ & 500 & SR & 16507 & process & 6319 & Oxalate & 495.459741 & 2.38 & 2.31 & 1179 & 1143 \\
\hline $09 / 29 / 2009$ & 500 & SR & 16507 & process & 6319 & Phosphate & 495.459741 & & . & . & -1 \\
\hline $09 / 29 / 2009$ & 500 & SR & 16507 & process & 6326 & Fluoride & 505.735225 & & & & \\
\hline $09 / 29 / 2009$ & 500 & SR & 16507 & process & 6326 & Formate & 505.735225 & 14.27 & 14.43 & 7219 & 7297 \\
\hline $09 / 29 / 2009$ & 500 & SR & 16507 & process & 6326 & $\begin{array}{l}\text { Chloride } \\
\end{array}$ & 505.735225 & & & & \\
\hline $09 / 29 / 2009$ & 500 & SR & 16507 & process & 6326 & Nitrite & 505.735225 & 17.7 & 17.64 & 8953 & 8920 \\
\hline $09 / 29 / 2009$ & 500 & SR & 16507 & process & 6326 & Nitrate & 505.735225 & 18.57 & 18.49 & 9391 & 9350 \\
\hline $09 / 29 / 2009$ & 500 & SR & 16507 & process & 6326 & Sulfate & 505.735225 & 1.2 & 1.16 & 605 & 588 \\
\hline $09 / 29 / 2009$ & 500 & SR & 16507 & process & 6326 & Oxalate & 505.735225 & 2.38 & 2.31 & 1201 & 1168 \\
\hline $09 / 29 / 2009$ & 500 & SR & 16507 & process & 6326 & Phosphate & 505.735225 & & & & \\
\hline $09 / 29 / 2009$ & 500 & SR & 16507 & standard & $2 \mathrm{ppm}$ & $\begin{array}{l}\text { Fluoride } \\
\end{array}$ & 1 & 1.98 & 1.99 & 1.98 & 1.99 \\
\hline $09 / 29 / 2009$ & 500 & SR & 16507 & standard & $2 \mathrm{ppm}$ & Formate & 1 & 2 & 2.01 & 2 & 2.01 \\
\hline $09 / 29 / 2009$ & 500 & SR & 16507 & standard & $2 \mathrm{ppm}$ & $\begin{array}{l}\text { Chloride } \\
\end{array}$ & 1 & 1.95 & 1.89 & 1.95 & 1.89 \\
\hline $09 / 29 / 2009$ & 500 & SR & 16507 & standard & $2 \mathrm{ppm}$ & Nitrite & 1 & 1.97 & 2 & 1.97 & 2 \\
\hline $09 / 29 / 2009$ & 500 & SR & 16507 & standard & $2 \mathrm{ppm}$ & Nitrate & 1 & 1.91 & 1.83 & 1.91 & 1.83 \\
\hline $09 / 29 / 2009$ & 500 & SR & 16507 & standard & $2 \mathrm{ppm}$ & Sulfate & 1 & 1.98 & 1.91 & 1.98 & 1.91 \\
\hline $09 / 29 / 2009$ & 500 & SR & 16507 & standard & $2 \mathrm{ppm}$ & Oxalate & 1 & 2.12 & 2.06 & 2.12 & 2.06 \\
\hline $09 / 29 / 2009$ & 500 & SR & 16507 & standard & $2 \mathrm{ppm}$ & Phosphate & 1 & 1.95 & 1.86 & 1.95 & 1.86 \\
\hline $09 / 29 / 2009$ & 500 & SR & 16507 & standard & $16 \mathrm{ppm}$ & Fluoride & 1 & 16.15 & 16.22 & 16.15 & 16.22 \\
\hline $\begin{array}{l}09 / 29 / 2009 \\
\end{array}$ & 500 & SR & 16507 & standard & $16 \mathrm{ppm}$ & Formate & 1 & 16.25 & 16.41 & 16.25 & 16.41 \\
\hline $09 / 29 / 2009$ & 500 & SR & 16507 & standard & $16 \mathrm{ppm}$ & $\begin{array}{l}\text { Chloride } \\
\end{array}$ & 1 & 16.16 & 16.16 & 16.16 & 16.16 \\
\hline $09 / 29 / 2009$ & 500 & SR & 16507 & standard & $16 \mathrm{ppm}$ & Nitrite & 1 & 16.12 & 16.28 & 16.12 & 16.28 \\
\hline
\end{tabular}


Table A1. Measurements of Standards and SRAT Receipt (SR), SRAT Product (SP), and SME Samples

\begin{tabular}{|c|c|c|c|c|c|c|c|c|c|c|c|}
\hline Date & $\begin{array}{c}\begin{array}{c}\text { Nominal } \\
\text { DF }\end{array} \\
\end{array}$ & Tank & $\begin{array}{c}\text { Sample } \\
\text { Number }\end{array}$ & $\begin{array}{l}\text { Type of } \\
\text { Sample }\end{array}$ & $\begin{array}{c}\text { Bottle } \\
\text { ID }\end{array}$ & Anion & DF & $\begin{array}{c}\text { M-13 ICS3000 } \\
\text { raw (ppm) }\end{array}$ & $\begin{array}{c}\text { M-14 ICS3000 } \\
\text { raw (ppm) }\end{array}$ & $\begin{array}{l}\text { M-13 ICS3000 } \\
\text { FINAL (ppm) } \\
\end{array}$ & $\begin{array}{l}\text { M-14 ICS3000 } \\
\text { FINAL (ppm) } \\
\end{array}$ \\
\hline $09 / 29 / 2009$ & 500 & SR & 16507 & standard & $16 \mathrm{ppm}$ & Nitrate & 1 & 16.27 & 16.25 & 16.27 & 16.25 \\
\hline $09 / 29 / 2009$ & 500 & SR & 16507 & standard & $16 \mathrm{ppm}$ & Sulfate & 1 & 16.18 & 16 & 16.18 & 16 \\
\hline $09 / 29 / 2009$ & 500 & SR & 16507 & standard & $16 \mathrm{ppm}$ & Oxalate & 1 & 16.25 & 16.39 & 16.25 & 16.39 \\
\hline $09 / 29 / 2009$ & 500 & SR & 16507 & standard & $16 \mathrm{ppm}$ & Phosphate & 1 & 15.92 & 15.43 & 15.92 & 15.43 \\
\hline $09 / 18 / 2009$ & . & SP & 16300 & standard & $2 \mathrm{ppm}$ & $\begin{array}{l}\text { Fluoride } \\
\end{array}$ & 1 & 2.02 & 2.02 & 2.02 & 2.02 \\
\hline $09 / 18 / 2009$ & . & SP & 16300 & standard & $2 \mathrm{ppm}$ & Formate & 1 & 2.01 & 1.98 & 2.01 & 1.98 \\
\hline $09 / 18 / 2009$ & . & SP & 16300 & standard & $2 \mathrm{ppm}$ & Chloride & 1 & 1.99 & 1.9 & 1.99 & 1.9 \\
\hline $09 / 18 / 2009$ & . & SP & 16300 & standard & $2 \mathrm{ppm}$ & Nitrite & 1 & 2.03 & 2.02 & 2.03 & 2.02 \\
\hline $09 / 18 / 2009$ &. & SP & 16300 & standard & $2 \mathrm{ppm}$ & Nitrate & 1 & 2 & 1.86 & 2 & 1.86 \\
\hline $09 / 18 / 2009$ & . & SP & 16300 & standard & $2 \mathrm{ppm}$ & Sulfate & 1 & 2.04 & 1.94 & 2.04 & 1.94 \\
\hline $09 / 18 / 2009$ & . & SP & 16300 & standard & $2 \mathrm{ppm}$ & Oxalate & 1 & 2.15 & 2.07 & 2.15 & 2.07 \\
\hline $09 / 18 / 2009$ & . & SP & 16300 & standard & $2 \mathrm{ppm}$ & Phosphate & 1 & 2.21 & 1.96 & 2.21 & 1.96 \\
\hline $09 / 18 / 2009$ & . & SP & 16300 & standard & $16 \mathrm{ppm}$ & Fluoride & 1 & 16.14 & 16.17 & 16.14 & 16.17 \\
\hline $09 / 18 / 2009$ & . & SP & 16300 & standard & $16 \mathrm{ppm}$ & Formate & 1 & 16.05 & 16.04 & 16.05 & 16.04 \\
\hline $09 / 18 / 2009$ & . & SP & 16300 & standard & $16 \mathrm{ppm}$ & Chloride & 1 & 16.17 & 16.12 & 16.17 & 16.12 \\
\hline $09 / 18 / 2009$ & . & SP & 16300 & standard & $16 \mathrm{ppm}$ & Nitrite & 1 & 16.45 & 16.3 & 16.45 & 16.3 \\
\hline $09 / 18 / 2009$ &. & SP & 16300 & standard & $16 \mathrm{ppm}$ & Nitrate & 1 & 16.45 & 16.15 & 16.45 & 16.15 \\
\hline $09 / 18 / 2009$ & . & SP & 16300 & standard & $16 \mathrm{ppm}$ & Sulfate & 1 & 16.35 & 16.08 & 16.35 & 16.08 \\
\hline $09 / 18 / 2009$ & . & SP & 16300 & standard & $16 \mathrm{ppm}$ & Oxalate & 1 & 16.12 & 16.14 & 16.12 & 16.14 \\
\hline $09 / 18 / 2009$ & - & SP & 16300 & standard & $16 \mathrm{ppm}$ & Phosphate & 1 & 16.02 & 15.53 & 16.02 & 15.53 \\
\hline $09 / 18 / 2009$ & 5000 & SP & 16300 & process & 5586 & Fluoride & 5056.51438 & & & & \\
\hline $09 / 18 / 2009$ & 5000 & SP & 16300 & process & 5586 & Formate & 5056.51438 & 8.04 & 7.88 & 40647 & 39821 \\
\hline $09 / 18 / 2009$ & 5000 & SP & 16300 & process & 5586 & Chloride & 5056.51438 & & & & \\
\hline $09 / 18 / 2009$ & 5000 & SP & 16300 & process & 5586 & Nitrite & 5056.51438 & & & & \\
\hline $09 / 18 / 2009$ & 5000 & SP & 16300 & process & 5586 & Nitrate & 5056.51438 & 4.23 & 4.07 & 21389 & 20593 \\
\hline $09 / 18 / 2009$ & 5000 & SP & 16300 & process & 5586 & Sulfate & 5056.51438 & &. & & \\
\hline $09 / 18 / 2009$ & 5000 & SP & 16300 & process & 5586 & Oxalate & 5056.51438 & . & . & - & . \\
\hline $09 / 18 / 2009$ & 5000 & SP & 16300 & process & 5586 & Phosphate & 5056.51438 & . & . & . & . \\
\hline $09 / 18 / 2009$ & 5000 & SP & 16300 & process & 5587 & Fluoride & 4908.78064 & & & & \\
\hline $09 / 18 / 2009$ & 5000 & SP & 16300 & process & 5587 & Formate & 4908.78064 & 8.2 & 8.05 & 40268 & 39516 \\
\hline $09 / 18 / 2009$ & 5000 & SP & 16300 & process & 5587 & Chloride & 4908.78064 & . & . & . & . \\
\hline $09 / 18 / 2009$ & 5000 & SP & 16300 & process & 5587 & Nitrite & 4908.78064 & & . & & \\
\hline $09 / 18 / 2009$ & 5000 & SP & 16300 & process & 5587 & Nitrate & 4908.78064 & 4.34 & 4.21 & 21280 & 20651 \\
\hline $09 / 18 / 2009$ & 5000 & SP & 16300 & process & 5587 & Sulfate & 4908.78064 & . & . & . &. \\
\hline $09 / 18 / 2009$ & 5000 & SP & 16300 & process & 5587 & Oxalate & 4908.78064 & &. & & \\
\hline $09 / 18 / 2009$ & 5000 & SP & 16300 & process & 5587 & Phosphate & 4908.78064 & & - & & \\
\hline $09 / 18 / 2009$ & 5000 & SP & 16300 & process & 5588 & Fluoride & 5099.93806 & & & & \\
\hline $09 / 18 / 2009$ & 5000 & SP & 16300 & process & 5588 & Formate & 5099.93806 & 7.95 & 7.81 & 40537 & 39812 \\
\hline $09 / 18 / 2009$ & 5000 & SP & 16300 & process & 5588 & Chloride & 5099.93806 & & - & & \\
\hline $09 / 18 / 2009$ & 5000 & SP & 16300 & process & 5588 & Nitrite & 5099.93806 & & & & \\
\hline $09 / 18 / 2009$ & 5000 & SP & 16300 & process & 5588 & Nitrate & 5099.93806 & 4.21 & 4.08 & 21488 & 20793 \\
\hline $09 / 18 / 2009$ & 5000 & SP & 16300 & process & 5588 & Sulfate & 5099.93806 & 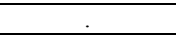 &. & & 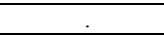 \\
\hline $09 / 18 / 2009$ & 5000 & SP & 16300 & process & 5588 & Oxalate & 5099.93806 & & . & & \\
\hline $09 / 18 / 2009$ & 5000 & SP & 16300 & process & 5588 & Phosphate & 5099.93806 & & . & & \\
\hline $09 / 18 / 2009$ & 5000 & SP & 16300 & process & 5589 & Fluoride & 4934.77835 & & & & \\
\hline $09 / 18 / 2009$ & 5000 & SP & 16300 & process & 5589 & Formate & 4934.77835 & 8.18 & 8.07 & 40360 & 39821 \\
\hline $09 / 18 / 2009$ & 5000 & SP & 16300 & process & 5589 & Chloride & 4934.77835 & & $\therefore$ & & \\
\hline $09 / 18 / 2009$ & 5000 & SP & 16300 & process & 5589 & Nitrite & 4934.77835 & & & & \\
\hline $09 / 18 / 2009$ & 5000 & SP & 16300 & process & 5589 & Nitrate & 4934.77835 & 4.35 & 4.19 & 21480 & 20683 \\
\hline $09 / 18 / 2009$ & 5000 & SP & 16300 & process & 5589 & Sulfate & 4934.77835 & & 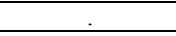 & & \\
\hline $09 / 18 / 2009$ & 5000 & SP & 16300 & process & 5589 & Oxalate & 4934.77835 & & & & \\
\hline
\end{tabular}


Table A1. Measurements of Standards and SRAT Receipt (SR), SRAT Product (SP), and SME Samples

\begin{tabular}{|c|c|c|c|c|c|c|c|c|c|c|c|}
\hline Date & $\begin{array}{c}\text { Nominal } \\
\text { DF }\end{array}$ & Tank & $\begin{array}{c}\text { Sample } \\
\text { Number }\end{array}$ & $\begin{array}{l}\text { Type of } \\
\text { Sample }\end{array}$ & $\begin{array}{c}\text { Bottle } \\
\text { ID }\end{array}$ & Anion & DF & $\begin{array}{l}\text { M-13 ICS3000 } \\
\text { raw (ppm) }\end{array}$ & $\begin{array}{l}\text { M-14 ICS3000 } \\
\text { raw (ppm) }\end{array}$ & $\begin{array}{l}\text { M-13 ICS3000 } \\
\text { FINAL (ppm) } \\
\end{array}$ & $\begin{array}{l}\text { M-14 ICS3000 } \\
\text { FINAL (ppm) }\end{array}$ \\
\hline $\begin{array}{l}09 / 18 / 2009 \\
\end{array}$ & 5000 & SP & 16300 & process & 5589 & Phosphate & 4934.77835 & & & & \\
\hline $09 / 18 / 2009$ & 16 & SP & 16300 & standard & 9999 & $\begin{array}{l}\text { Fluoride } \\
\end{array}$ & 2 & 8.1 & 8.08 & 101.29 & 101.01 \\
\hline $09 / 18 / 2009$ & 16 & SP & 16300 & standard & 9999 & Formate & 2 & 12.1 & 12.07 & 100.12 & 100.45 \\
\hline $\begin{array}{l}09 / 18 / 2009 \\
\end{array}$ & 16 & SP & 16300 & standard & 9999 & $\begin{array}{l}\text { Chloride } \\
\end{array}$ & 2 & 8.1 & 8 & 101.24 & 99.95 \\
\hline $09 / 18 / 2009$ & 16 & SP & 16300 & standard & 9999 & Nitrite & 2 & 8.2 & 8.15 & 102.45 & 101.83 \\
\hline $09 / 18 / 2009$ & 16 & SP & 16300 & standard & 9999 & Nitrate & 2 & 10.44 & 10.26 & 103.34 & 102.06 \\
\hline $09 / 18 / 2009$ & 16 & SP & 16300 & standard & 9999 & Sulfate & 2 & 8.27 & 8.06 & 103.35 & 100.75 \\
\hline $09 / 18 / 2009$ & 16 & SP & 16300 & standard & 9999 & Oxalate & 2 & 8.23 & 8.18 & 102.85 & 102.2 \\
\hline $\begin{array}{l}09 / 18 / 2009 \\
\end{array}$ & 16 & SP & 16300 & standard & 9999 & Phosphate & 2 & 8.02 & 7.71 & 100.21 & 96.32 \\
\hline $\begin{array}{l}09 / 18 / 2009 \\
\end{array}$ & 500 & SP & 16300 & process & 5579 & $\begin{array}{l}\text { Fluoride } \\
\end{array}$ & 507.15014 & & & & \\
\hline $09 / 18 / 2009$ & 500 & SP & 16300 & process & 5579 & Formate & 507.15014 & 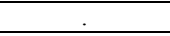 & . & 0 & 0 \\
\hline $09 / 18 / 2009$ & 500 & SP & 16300 & process & 5579 & Chloride & 507.15014 & & . & 0 & 0 \\
\hline $09 / 18 / 2009$ & 500 & SP & 16300 & process & 5579 & Nitrite & 507.15014 & & . & & \\
\hline $\begin{array}{l}09 / 18 / 2009 \\
\end{array}$ & 500 & SP & 16300 & process & 5579 & Nitrate & 507.15014 & & & & \\
\hline $09 / 18 / 2009$ & 500 & SP & 16300 & process & 5579 & Sulfate & 507.15014 & 1.26 & 1.22 & 637 & 621 \\
\hline $09 / 18 / 2009$ & 500 & SP & 16300 & process & 5579 & Oxalate & 507.15014 & . & . & . & . \\
\hline $\begin{array}{l}09 / 18 / 2009 \\
\end{array}$ & 500 & SP & 16300 & process & 5579 & Phosphate & 507.15014 & &. & 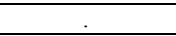 & \\
\hline $09 / 18 / 2009$ & 500 & SP & 16300 & process & 5580 & $\begin{array}{l}\text { Fluoride } \\
\end{array}$ & 495.912676 & & . & & \\
\hline $09 / 18 / 2009$ & 500 & SP & 16300 & process & 5580 & Formate & 495.912676 & & - & 0 & 0 \\
\hline $09 / 18 / 2009$ & 500 & SP & 16300 & process & 5580 & $\begin{array}{l}\text { Chloride } \\
\end{array}$ & 495.912676 & . & . & 0 & 0 \\
\hline $09 / 18 / 2009$ & 500 & SP & 16300 & process & 5580 & Nitrite & 495.912676 & . & . & . & . \\
\hline $09 / 18 / 2009$ & 500 & SP & 16300 & process & 5580 & Nitrate & 495.912676 & & & & \\
\hline $\begin{array}{l}09 / 18 / 2009 \\
\end{array}$ & 500 & SP & 16300 & process & 5580 & Sulfate & 495.912676 & 1.27 & 1.26 & 632 & 623 \\
\hline $09 / 18 / 2009$ & 500 & $\begin{array}{l}\mathrm{SP} \\
\end{array}$ & 16300 & process & 5580 & Oxalate & 495.912676 & &. &. & \\
\hline $09 / 18 / 2009$ & 500 & SP & 16300 & process & 5580 & Phosphate & 495.912676 & & 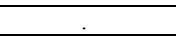 & & \\
\hline $\begin{array}{l}09 / 18 / 2009 \\
\end{array}$ & 500 & $\begin{array}{l}\text { SP } \\
\end{array}$ & 16300 & process & 5581 & $\begin{array}{l}\text { Fluoride } \\
\end{array}$ & 508.733471 & & . & & \\
\hline $09 / 18 / 2009$ & 500 & SP & 16300 & process & 5581 & Formate & 508.733471 & . & . & 0 & 0 \\
\hline $09 / 18 / 2009$ & 500 & SP & 16300 & process & 5581 & $\begin{array}{l}\text { Chloride } \\
\end{array}$ & 508.733471 & . & . & 0 & 0 \\
\hline $09 / 18 / 2009$ & 500 & SP & 16300 & process & 5581 & Nitrite & 508.733471 & & . & . & \\
\hline $09 / 18 / 2009$ & 500 & SP & 16300 & process & 5581 & Nitrate & 508.733471 & & & & \\
\hline $09 / 18 / 2009$ & 500 & SP & 16300 & process & 5581 & Sulfate & 508.733471 & 1.24 & 1.21 & 631 & 617 \\
\hline $09 / 18 / 2009$ & 500 & SP & 16300 & process & 5581 & Oxalate & 508.733471 & & $\dot{\square}$ &. & \\
\hline $\begin{array}{l}09 / 18 / 2009 \\
\end{array}$ & 500 & SP & 16300 & process & 5581 & Phosphate & 508.733471 & . &. & . & . \\
\hline $09 / 18 / 2009$ & 500 & SP & 16300 & process & 5582 & $\begin{array}{l}\text { Fluoride } \\
\end{array}$ & 504.143535 & . & . & . & . \\
\hline $09 / 18 / 2009$ & 500 & SP & 16300 & process & 5582 & Formate & 504.143535 & & . & 0 & 0 \\
\hline $\begin{array}{l}09 / 18 / 2009 \\
\end{array}$ & 500 & SP & 16300 & process & 5582 & $\begin{array}{l}\text { Chloride } \\
\end{array}$ & 504.143535 & & 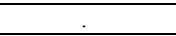 & 0 & 0 \\
\hline $09 / 18 / 2009$ & 500 & SP & 16300 & process & 5582 & Nitrite & 504.143535 & &. & $\dot{\square}$ & \\
\hline $09 / 18 / 2009$ & 500 & SP & 16300 & process & 5582 & Nitrate & 504.143535 & & & & \\
\hline $\begin{array}{l}09 / 18 / 2009 \\
\end{array}$ & 500 & SP & 16300 & process & 5582 & Sulfate & 504.143535 & 1.25 & 1.23 & 628 & 622 \\
\hline $09 / 18 / 2009$ & 500 & SP & 16300 & process & 5582 & Oxalate & 504.143535 & 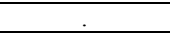 & $\cdot$ & . & $\cdot$ \\
\hline $09 / 18 / 2009$ & 500 & SP & 16300 & process & 5582 & Phosphate & 504.143535 & & & & \\
\hline $\begin{array}{l}09 / 18 / 2009 \\
\end{array}$ & 500 & SR & 16300 & standard & $2 \mathrm{ppm}$ & $\begin{array}{l}\text { Fluoride } \\
\end{array}$ & 1 & 2.02 & 2.01 & 2.02 & 2.01 \\
\hline $09 / 18 / 2009$ & 500 & SR & 16300 & standard & $2 \mathrm{ppm}$ & Formate & 1 & 2.01 & 2.04 & 2.01 & 2.04 \\
\hline $09 / 18 / 2009$ & 500 & SR & 16300 & standard & $2 \mathrm{ppm}$ & Chloride & 1 & 1.97 & 1.89 & 1.97 & 1.89 \\
\hline $09 / 18 / 2009$ & 500 & SR & 16300 & standard & $2 \mathrm{ppm}$ & Nitrite & 1 & 2.02 & 2.03 & 2.02 & 2.03 \\
\hline $09 / 18 / 2009$ & 500 & SR & 16300 & standard & $2 \mathrm{ppm}$ & Nitrate & 1 & 1.94 & 1.97 & 1.94 & 1.97 \\
\hline $09 / 18 / 2009$ & 500 & SR & 16300 & standard & $2 \mathrm{ppm}$ & Sulfate & 1 & 1.98 & 1.93 & 1.98 & 1.93 \\
\hline $09 / 18 / 2009$ & 500 & SR & 16300 & standard & $2 \mathrm{ppm}$ & Oxalate & 1 & 2.08 & 2.07 & 2.08 & 2.07 \\
\hline $\begin{array}{l}09 / 18 / 2009 \\
\end{array}$ & 500 & SR & 16300 & standard & $2 \mathrm{ppm}$ & Phosphate & 1 & 2.04 & 1.89 & 2.04 & 1.89 \\
\hline $09 / 18 / 2009$ & 500 & SR & 16300 & standard & $16 \mathrm{ppm}$ & $\begin{array}{l}\text { Fluoride } \\
\end{array}$ & 1 & 16.15 & 16.19 & 16.15 & 16.19 \\
\hline $09 / 18 / 2009$ & 500 & SR & 16300 & standard & $16 \mathrm{ppm}$ & Formate & 1 & 16.16 & 16.23 & 16.16 & 16.23 \\
\hline
\end{tabular}


Table A1. Measurements of Standards and SRAT Receipt (SR), SRAT Product (SP), and SME Samples

\begin{tabular}{|c|c|c|c|c|c|c|c|c|c|c|c|}
\hline Date & $\begin{array}{c}\begin{array}{c}\text { Nominal } \\
\text { DF }\end{array} \\
\end{array}$ & Tank & $\begin{array}{c}\text { Sample } \\
\text { Number }\end{array}$ & $\begin{array}{l}\text { Type of } \\
\text { Sample }\end{array}$ & $\begin{array}{c}\text { Bottle } \\
\text { ID }\end{array}$ & Anion & DF & $\begin{array}{c}\text { M-13 ICS3000 } \\
\text { raw (ppm) }\end{array}$ & $\begin{array}{c}\text { M-14 ICS3000 } \\
\text { raw (ppm) }\end{array}$ & $\begin{array}{l}\text { M-13 ICS3000 } \\
\text { FINAL (ppm) } \\
\end{array}$ & $\begin{array}{l}\text { M-14 ICS3000 } \\
\text { FINAL (ppm) } \\
\end{array}$ \\
\hline $09 / 18 / 2009$ & 500 & SR & 16300 & standard & $16 \mathrm{ppm}$ & Chloride & 1 & 16.16 & 16.14 & 16.16 & 16.14 \\
\hline $09 / 18 / 2009$ & 500 & SR & 16300 & standard & $16 \mathrm{ppm}$ & Nitrite & 1 & 16.53 & 16.52 & 16.53 & 16.52 \\
\hline $09 / 18 / 2009$ & 500 & SR & 16300 & standard & $16 \mathrm{ppm}$ & Nitrate & 1 & 16.34 & 16.16 & 16.34 & 16.16 \\
\hline $09 / 18 / 2009$ & 500 & SR & 16300 & standard & $16 \mathrm{ppm}$ & Sulfate & 1 & 16.23 & 16.09 & 16.23 & 16.09 \\
\hline $09 / 18 / 2009$ & 500 & SR & 16300 & standard & $16 \mathrm{ppm}$ & Oxalate & 1 & 16.07 & 16.24 & 16.07 & 16.24 \\
\hline $09 / 18 / 2009$ & 500 & SR & 16300 & standard & $16 \mathrm{ppm}$ & Phosphate & 1 & 15.66 & 15.559 & 15.66 & 15.56 \\
\hline $09 / 25 / 2009$ & . & SP & 16437 & standard & $2 \mathrm{ppm}$ & Fluoride & 1 & 2 & 2.04 & 2 & 2.04 \\
\hline $09 / 25 / 2009$ & . & SP & 16437 & standard & $2 \mathrm{ppm}$ & Formate & 1 & 2.01 & 2.05 & 2.01 & 2.05 \\
\hline $09 / 25 / 2009$ &. & SP & 16437 & standard & $2 \mathrm{ppm}$ & Chloride & 1 & 1.96 & 1.94 & 1.96 & 1.94 \\
\hline $09 / 25 / 2009$ & . & SP & 16437 & standard & $2 \mathrm{ppm}$ & Nitrite & 1 & 1.98 & 2.03 & 1.98 & 2.03 \\
\hline $09 / 25 / 2009$ & . & SP & 16437 & standard & $2 \mathrm{ppm}$ & Nitrate & 1 & 1.96 & 1.88 & 1.96 & 1.88 \\
\hline $09 / 25 / 2009$ & . & SP & 16437 & standard & $2 \mathrm{ppm}$ & Sulfate & 1 & 2.01 & 1.94 & 2.01 & 1.94 \\
\hline $09 / 25 / 2009$ & . & SP & 16437 & standard & $2 \mathrm{ppm}$ & Oxalate & 1 & 2.15 & 2.1 & 2.15 & 2.1 \\
\hline $09 / 25 / 2009$ & . & SP & 16437 & standard & $2 \mathrm{ppm}$ & Phosphate & 1 & 2.08 & 2.01 & 2.08 & 2.01 \\
\hline $09 / 25 / 2009$ & . & SP & 16437 & standard & $16 \mathrm{ppm}$ & Fluoride & 1 & 15.88 & 16.19 & 15.88 & 16.19 \\
\hline $09 / 25 / 2009$ & . & SP & 16437 & standard & $16 \mathrm{ppm}$ & Formate & 1 & 15.93 & 16.28 & 15.93 & 16.28 \\
\hline $09 / 25 / 2009$ & . & SP & 16437 & standard & $16 \mathrm{ppm}$ & Chloride & 1 & 15.94 & 16.17 & 15.94 & 16.17 \\
\hline $09 / 25 / 2009$ & . & SP & 16437 & standard & $16 \mathrm{ppm}$ & Nitrite & 1 & 15.56 & 15.9 & 15.56 & 15.9 \\
\hline $09 / 25 / 2009$ & . & SP & 16437 & standard & $16 \mathrm{ppm}$ & Nitrate & 1 & 16.12 & 16.29 & 16.12 & 16.29 \\
\hline $09 / 25 / 2009$ &. & SP & 16437 & standard & $16 \mathrm{ppm}$ & Sulfate & 1 & 16.03 & 16.01 & 16.03 & 16.01 \\
\hline $09 / 25 / 2009$ &. & SP & 16437 & standard & $16 \mathrm{ppm}$ & Oxalate & 1 & 16.01 & 16.29 & 16.01 & 16.29 \\
\hline $09 / 25 / 2009$ & & SP & 16437 & standard & $16 \mathrm{ppm}$ & Phosphate & 1 & 15.92 & 15.67 & 15.92 & 15.67 \\
\hline $09 / 25 / 2009$ & 5000 & SP & 16437 & process & 6159 & $\begin{array}{l}\text { Fluoride } \\
\end{array}$ & 4741.59556 & & & & \\
\hline $09 / 25 / 2009$ & 5000 & SP & 16437 & process & 6159 & Formate & 4741.59556 & 8.31 & 8.7 & 39405 & 41258 \\
\hline $09 / 25 / 2009$ & 5000 & SP & 16437 & process & 6159 & Chloride & 4741.59556 & &. & & \\
\hline $09 / 25 / 2009$ & 5000 & SP & 16437 & process & 6159 & Nitrite & 4741.59556 & & & & \\
\hline $09 / 25 / 2009$ & 5000 & SP & 16437 & process & 6159 & Nitrate & 4741.59556 & 4.66 & 4.68 & 22088 & 22174 \\
\hline $09 / 25 / 2009$ & 5000 & SP & 16437 & process & 6159 & Sulfate & 4741.59556 & . & . & . & . \\
\hline $09 / 25 / 2009$ & 5000 & SP & 16437 & process & 6159 & Oxalate & 4741.59556 & - & . & & . \\
\hline $09 / 25 / 2009$ & 5000 & SP & 16437 & process & 6159 & Phosphate & 4741.59556 & 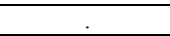 & . & . & . \\
\hline $09 / 25 / 2009$ & 5000 & SP & 16437 & process & 6160 & Fluoride & 4806.68425 & & . & & \\
\hline $09 / 25 / 2009$ & 5000 & SP & 16437 & process & 6160 & Formate & 4806.68425 & 8.18 & 8.41 & 39321 & 40422 \\
\hline $09 / 25 / 2009$ & 5000 & SP & 16437 & process & 6160 & Chloride & 4806.68425 &. & . & . &. \\
\hline $09 / 25 / 2009$ & 5000 & SP & 16437 & process & 6160 & Nitrite & 4806.68425 & & . & & \\
\hline $09 / 25 / 2009$ & 5000 & SP & 16437 & process & 6160 & Nitrate & 4806.68425 & 4.63 & 4.57 & 22255 & 21958 \\
\hline $09 / 25 / 2009$ & 5000 & SP & 16437 & process & 6160 & Sulfate & 4806.68425 & & . & & \\
\hline $09 / 25 / 2009$ & 5000 & SP & 16437 & process & 6160 & Oxalate & 4806.68425 & & . & & \\
\hline $09 / 25 / 2009$ & 5000 & SP & 16437 & process & 6160 & Phosphate & 4806.68425 & & . & & \\
\hline $09 / 25 / 2009$ & 5000 & SP & 16437 & process & 6161 & Fluoride & 4870.76921 & & & & \\
\hline $09 / 25 / 2009$ & 5000 & SP & 16437 & process & 6161 & Formate & 4870.76921 & 8.09 & 8.46 & 39428 & 41216 \\
\hline $09 / 25 / 2009$ & 5000 & SP & 16437 & process & 6161 & Chloride & 4870.76921 & & . & $\dot{\square}$ & $\dot{.}$ \\
\hline $09 / 25 / 2009$ & 5000 & SP & 16437 & process & 6161 & Nitrite & 4870.76921 & & & & \\
\hline $09 / 25 / 2009$ & 5000 & SP & 16437 & process & 6161 & Nitrate & 4870.76921 & 4.55 & 4.49 & 22175 & 21871 \\
\hline $09 / 25 / 2009$ & 5000 & SP & 16437 & process & 6161 & Sulfate & 4870.76921 & 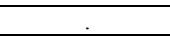 & . & & - \\
\hline $09 / 25 / 2009$ & 5000 & SP & 16437 & process & 6161 & Oxalate & 4870.76921 & 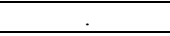 & . & . & . \\
\hline $09 / 25 / 2009$ & 5000 & SP & 16437 & process & 6161 & Phosphate & 4870.76921 & . & . & . & . \\
\hline $09 / 25 / 2009$ & 5000 & SP & 16437 & process & 6162 & Fluoride & 4920.59689 & & & & \\
\hline $09 / 25 / 2009$ & 5000 & SP & 16437 & process & 6162 & Formate & 4920.59689 & 8.05 & 8.37 & 39609 & 41171 \\
\hline $09 / 25 / 2009$ & 5000 & SP & 16437 & process & 6162 & Chloride & 4920.59689 & &. & & \\
\hline $09 / 25 / 2009$ & 5000 & SP & 16437 & process & 6162 & Nitrite & 4920.59689 & & & & \\
\hline $09 / 25 / 2009$ & 5000 & SP & 16437 & process & 6162 & Nitrate & 4920.59689 & 4.52 & 4.47 & 22247 & 21978 \\
\hline
\end{tabular}


Table A1. Measurements of Standards and SRAT Receipt (SR), SRAT Product (SP), and SME Samples

\begin{tabular}{|c|c|c|c|c|c|c|c|c|c|c|c|}
\hline Date & $\begin{array}{c}\begin{array}{c}\text { Nominal } \\
\text { DF }\end{array} \\
\end{array}$ & Tank & $\begin{array}{c}\text { Sample } \\
\text { Number }\end{array}$ & $\begin{array}{l}\text { Type of } \\
\text { Sample }\end{array}$ & $\begin{array}{c}\text { Bottle } \\
\text { ID }\end{array}$ & Anion & DF & $\begin{array}{c}\text { M-13 ICS3000 } \\
\text { raw (ppm) }\end{array}$ & $\begin{array}{c}\text { M-14 ICS3000 } \\
\text { raw (ppm) }\end{array}$ & $\begin{array}{l}\text { M-13 ICS3000 } \\
\text { FINAL (ppm) } \\
\end{array}$ & $\begin{array}{l}\text { M-14 ICS3000 } \\
\text { FINAL (ppm) } \\
\end{array}$ \\
\hline $09 / 25 / 2009$ & 5000 & SP & 16437 & process & 6162 & Sulfate & 4920.59689 & & & & \\
\hline $09 / 25 / 2009$ & 5000 & SP & 16437 & process & 6162 & Oxalate & 4920.59689 & & . & & \\
\hline $09 / 25 / 2009$ & 5000 & SP & 16437 & process & 6162 & Phosphate & 4920.59689 & & & & \\
\hline $09 / 25 / 2009$ & 16 & SP & 16437 & standard & 9999 & Fluoride & 2 & 8.05 & 8.1 & 100.63 & 101.25 \\
\hline $09 / 25 / 2009$ & 16 & SP & 16437 & standard & 9999 & Formate & 2 & 12.02 & 12.39 & 99.93 & 102.63 \\
\hline $09 / 25 / 2009$ & 16 & SP & 16437 & standard & 9999 & Chloride & 2 & 8.01 & 8 & 100.08 & 100.03 \\
\hline $09 / 25 / 2009$ & 16 & SP & 16437 & standard & 9999 & Nitrite & 2 & 7.83 & 7.93 & 97.86 & 99.11 \\
\hline $09 / 25 / 2009$ & 16 & SP & 16437 & standard & 9999 & Nitrate & 2 & 10.48 & 10.59 & 102.77 & 104.48 \\
\hline $09 / 25 / 2009$ & 16 & SP & 16437 & standard & 9999 & Sulfate & 2 & 8.16 & 8.03 & 101.96 & 100.35 \\
\hline $09 / 25 / 2009$ & 16 & SP & 16437 & standard & 9999 & Oxalate & 2 & 8.29 & 8.33 & 103.67 & 104.15 \\
\hline $09 / 25 / 2009$ & 16 & SP & 16437 & standard & 9999 & Phosphate & 2 & 8.1 & 7.76 & 101.21 & 97.01 \\
\hline $09 / 25 / 2009$ & 500 & SP & 16437 & process & 6149 & $\begin{array}{l}\text { Fluoride } \\
\end{array}$ & 488.881496 & & . & & \\
\hline $09 / 25 / 2009$ & 500 & SP & 16437 & process & 6149 & Formate & 488.881496 & 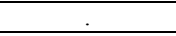 & . & & \\
\hline $09 / 25 / 2009$ & 500 & SP & 16437 & process & 6149 & Chloride & 488.881496 & 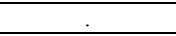 &. & & \\
\hline $09 / 25 / 2009$ & 500 & SP & 16437 & process & 6149 & Nitrite & 488.881496 & . & . & . & . \\
\hline $09 / 25 / 2009$ & 500 & SP & 16437 & process & 6149 & Nitrate & 488.881496 & & & & \\
\hline $09 / 25 / 2009$ & 500 & SP & 16437 & process & 6149 & Sulfate & 488.881496 & 1.33 & 1.31 & 652 & 640 \\
\hline $09 / 25 / 2009$ & 500 & SP & 16437 & process & 6149 & Oxalate & 488.881496 & . & . & . & . \\
\hline $09 / 25 / 2009$ & 500 & SP & 16437 & process & 6149 & Phosphate & 488.881496 & 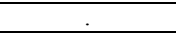 & . & & \\
\hline $09 / 25 / 2009$ & 500 & SP & 16437 & process & 6150 & Fluoride & 510.796075 &. &. &. &. \\
\hline $09 / 25 / 2009$ & 500 & SP & 16437 & process & 6150 & Formate & 510.796075 & . & . & . &. \\
\hline $09 / 25 / 2009$ & 500 & SP & 16437 & process & 6150 & Chloride & 510.796075 & . & . & . & . \\
\hline $09 / 25 / 2009$ & 500 & SP & 16437 & process & 6150 & Nitrite & 510.796075 & & . & & \\
\hline $09 / 25 / 2009$ & 500 & SP & 16437 & process & 6150 & Nitrate & 510.796075 & & 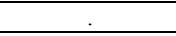 & & \\
\hline $09 / 25 / 2009$ & 500 & SP & 16437 & process & 6150 & Sulfate & 510.796075 & 1.35 & 1.31 & 688 & 667 \\
\hline $09 / 25 / 2009$ & 500 & SP & 16437 & process & 6150 & Oxalate & 510.796075 & &. & 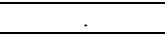 & \\
\hline $09 / 25 / 2009$ & 500 & SP & 16437 & process & 6150 & Phosphate & 510.796075 & . & . & . & . \\
\hline $09 / 25 / 2009$ & 500 & SP & 16437 & process & 6151 & Fluoride & 526.752581 & . & . & . & . \\
\hline $09 / 25 / 2009$ & 500 & SP & 16437 & process & 6151 & Formate & 526.752581 & . & . & - & . \\
\hline $09 / 25 / 2009$ & 500 & SP & 16437 & process & 6151 & Chloride & 526.752581 & . & . & . & . \\
\hline $09 / 25 / 2009$ & 500 & SP & 16437 & process & 6151 & Nitrite & 526.752581 & . & . & . & . \\
\hline $09 / 25 / 2009$ & 500 & SP & 16437 & process & 6151 & Nitrate & 526.752581 & &. & & \\
\hline $09 / 25 / 2009$ & 500 & SP & 16437 & process & 6151 & Sulfate & 526.752581 & 1.31 & 1.27 & 690 & 667 \\
\hline $09 / 25 / 2009$ & 500 & SP & 16437 & process & 6151 & Oxalate & 526.752581 & $\dot{\square}$ &. & . & . \\
\hline $09 / 25 / 2009$ & 500 & SP & 16437 & process & 6151 & Phosphate & 526.752581 & 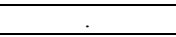 &. & & \\
\hline $09 / 25 / 2009$ & 500 & SP & 16437 & process & 6152 & Fluoride & 523.558005 & &. & & \\
\hline $09 / 25 / 2009$ & 500 & SP & 16437 & process & 6152 & Formate & 523.558005 & . & . & & . \\
\hline $09 / 25 / 2009$ & 500 & SP & 16437 & process & 6152 & Chloride & 523.558005 & & . & & \\
\hline $09 / 25 / 2009$ & 500 & SP & 16437 & process & 6152 & Nitrite & 523.558005 & & - & 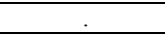 & \\
\hline $09 / 25 / 2009$ & 500 & SP & 16437 & process & 6152 & Nitrate & 523.558005 & & & & \\
\hline $09 / 25 / 2009$ & 500 & SP & 16437 & process & 6152 & Sulfate & 523.558005 & 1.33 & 1.29 & 698 & 676 \\
\hline $09 / 25 / 2009$ & 500 & SP & 16437 & process & 6152 & Oxalate & 523.558005 & &. & & \\
\hline $09 / 25 / 2009$ & 500 & SP & 16437 & process & 6152 & Phosphate & 523.558005 & & & & \\
\hline $09 / 25 / 2009$ & 500 & SR & 16437 & standard & $2 \mathrm{ppm}$ & $\begin{array}{l}\text { Fluoride } \\
\end{array}$ & 1 & 2.01 & 2.04 & 2.01 & 2.04 \\
\hline $09 / 25 / 2009$ & 500 & SR & 16437 & standard & $2 \mathrm{ppm}$ & Formate & 1 & 2.02 & 2.07 & 2.02 & 2.07 \\
\hline $09 / 25 / 2009$ & 500 & SR & 16437 & standard & $2 \mathrm{ppm}$ & Chloride & 1 & 1.97 & 1.93 & 1.97 & 1.93 \\
\hline $09 / 25 / 2009$ & 500 & SR & 16437 & standard & $2 \mathrm{ppm}$ & Nitrite & 1 & 1.98 & 2.04 & 1.98 & 2.04 \\
\hline $09 / 25 / 2009$ & 500 & SR & 16437 & standard & $2 \mathrm{ppm}$ & Nitrate & 1 & 1.96 & 1.9 & 1.96 & 1.9 \\
\hline $09 / 25 / 2009$ & 500 & SR & 16437 & standard & $2 \mathrm{ppm}$ & Sulfate & 1 & 2.01 & 1.96 & 2.01 & 1.96 \\
\hline $09 / 25 / 2009$ & 500 & SR & 16437 & standard & $2 \mathrm{ppm}$ & Oxalate & 1 & 2.16 & 2.12 & 2.16 & 2.12 \\
\hline $09 / 25 / 2009$ & 500 & SR & 16437 & standard & $2 \mathrm{ppm}$ & Phosphate & 1 & 2.07 & 1.99 & 2.07 & 1.99 \\
\hline
\end{tabular}


Table A1. Measurements of Standards and SRAT Receipt (SR), SRAT Product (SP), and SME Samples

\begin{tabular}{|c|c|c|c|c|c|c|c|c|c|c|c|}
\hline Date & $\begin{array}{c}\begin{array}{c}\text { Nominal } \\
\text { DF }\end{array} \\
\end{array}$ & Tank & $\begin{array}{c}\text { Sample } \\
\text { Number }\end{array}$ & $\begin{array}{l}\text { Type of } \\
\text { Sample }\end{array}$ & $\begin{array}{c}\text { Bottle } \\
\text { ID }\end{array}$ & Anion & DF & $\begin{array}{c}\text { M-13 ICS3000 } \\
\text { raw (ppm) }\end{array}$ & $\begin{array}{c}\text { M-14 ICS3000 } \\
\text { raw (ppm) }\end{array}$ & $\begin{array}{l}\text { M-13 ICS3000 } \\
\text { FINAL (ppm) } \\
\end{array}$ & $\begin{array}{l}\text { M-14 ICS3000 } \\
\text { FINAL (ppm) } \\
\end{array}$ \\
\hline $09 / 25 / 2009$ & 500 & SR & 16437 & standard & $16 \mathrm{ppm}$ & Fluoride & 1 & 15.94 & 16.17 & 15.94 & 16.17 \\
\hline $09 / 25 / 2009$ & 500 & SR & 16437 & standard & $16 \mathrm{ppm}$ & Formate & 1 & 16.08 & 16.41 & 16.08 & 16.41 \\
\hline $09 / 25 / 2009$ & 500 & SR & 16437 & standard & $16 \mathrm{ppm}$ & Chloride & 1 & 15.99 & 16.14 & 15.99 & 16.14 \\
\hline $09 / 25 / 2009$ & 500 & SR & 16437 & standard & $16 \mathrm{ppm}$ & Nitrite & 1 & 15.79 & 16.03 & 15.79 & 16.03 \\
\hline $09 / 25 / 2009$ & 500 & SR & 16437 & standard & $16 \mathrm{ppm}$ & Nitrate & 1 & 16.21 & 16.34 & 16.21 & 16.34 \\
\hline $09 / 25 / 2009$ & 500 & SR & 16437 & standard & $16 \mathrm{ppm}$ & Sulfate & 1 & 16.05 & 16 & 16.05 & 16 \\
\hline $09 / 25 / 2009$ & 500 & SR & 16437 & standard & $16 \mathrm{ppm}$ & Oxalate & 1 & 16.16 & 16.37 & 16.16 & 16.37 \\
\hline $09 / 25 / 2009$ & 500 & SR & 16437 & standard & $16 \mathrm{ppm}$ & Phosphate & 1 & 15.95 & 15.6098 & 15.95 & 15.61 \\
\hline $09 / 17 / 2009$ &. & SME & 16282 & standard & $2 \mathrm{ppm}$ & Fluoride & 1 & 2.03 & 2.03 & 2.03 & 2.03 \\
\hline $09 / 17 / 2009$ & . & SME & 16282 & standard & $2 \mathrm{ppm}$ & Formate & 1 & 2.04 & 2.04 & 2.04 & 2.04 \\
\hline $09 / 17 / 2009$ & . & SME & 16282 & standard & $2 \mathrm{ppm}$ & Chloride & 1 & 1.98 & 1.9 & 1.98 & 1.9 \\
\hline $09 / 17 / 2009$ & . & SME & 16282 & standard & $2 \mathrm{ppm}$ & Nitrite & 1 & 2.03 & 1.96 & 2.03 & 1.96 \\
\hline $09 / 17 / 2009$ & . & SME & 16282 & standard & $2 \mathrm{ppm}$ & Nitrate & 1 & 1.93 & 1.86 & 1.93 & 1.86 \\
\hline $09 / 17 / 2009$ & . & SME & 16282 & standard & $2 \mathrm{ppm}$ & Sulfate & 1 & 1.97 & 1.96 & 1.97 & 1.96 \\
\hline $09 / 17 / 2009$ & . & SME & 16282 & standard & $2 \mathrm{ppm}$ & Oxalate & 1 & 2.09 & 2.12 & 2.09 & 2.12 \\
\hline $09 / 17 / 2009$ & . & SME & 16282 & standard & $2 \mathrm{ppm}$ & Phosphate & 1 & 1.92 & 1.92 & 1.92 & 1.92 \\
\hline $09 / 17 / 2009$ &. & SME & 16282 & standard & $16 \mathrm{ppm}$ & $\begin{array}{l}\text { Fluoride } \\
\end{array}$ & 1 & 16.09 & 16.1 & 16.09 & 16.1 \\
\hline $09 / 17 / 2009$ & . & SME & 16282 & standard & $16 \mathrm{ppm}$ & Formate & 1 & 16.29 & 16.13 & 16.29 & 16.13 \\
\hline $09 / 17 / 2009$ & . & SME & 16282 & standard & $16 \mathrm{ppm}$ & Chloride & 1 & 16.15 & 16.12 & 16.15 & 16.12 \\
\hline $09 / 17 / 2009$ & . & SME & 16282 & standard & $16 \mathrm{ppm}$ & Nitrite & 1 & 16.41 & 16.34 & 16.41 & 16.34 \\
\hline $09 / 17 / 2009$ & . & SME & 16282 & standard & $16 \mathrm{ppm}$ & Nitrate & 1 & 16.3 & 16.31 & 16.3 & 16.31 \\
\hline $09 / 17 / 2009$ & . & SME & 16282 & standard & $16 \mathrm{ppm}$ & Sulfate & 1 & 16.13 & 16.04 & 16.13 & 16.04 \\
\hline $09 / 17 / 2009$ & & SME & 16282 & standard & $16 \mathrm{ppm}$ & Oxalate & 1 & 16.16 & 16.36 & 16.16 & 16.36 \\
\hline $09 / 17 / 2009$ & & $\begin{array}{l}\text { SME } \\
\end{array}$ & 16282 & standard & $16 \mathrm{ppm}$ & Phosphate & 1 & 15.67 & 15.67 & 15.67 & 15.67 \\
\hline $09 / 17 / 2009$ & 5000 & SME & 16282 & process & 5440 & $\begin{array}{l}\text { Fluoride } \\
\end{array}$ & 4904.50385 & & & & \\
\hline $09 / 17 / 2009$ & 5000 & $\begin{array}{l}\text { SME } \\
\end{array}$ & 16282 & process & 5440 & Formate & 4904.50385 & 6.63 & 6.79 & 32519 & 33323 \\
\hline $09 / 17 / 2009$ & 5000 & SME & 16282 & process & 5440 & Chloride & 4904.50385 & 5 & . & . & . \\
\hline $09 / 17 / 2009$ & 5000 & SME & 16282 & process & 5440 & Nitrite & 4904.50385 & & & & \\
\hline $09 / 17 / 2009$ & 5000 & SME & 16282 & process & 5440 & Nitrate & 4904.50385 & 3.62 & 3.44 & 17765 & 16860 \\
\hline $09 / 17 / 2009$ & 5000 & SME & 16282 & process & 5440 & Sulfate & 4904.50385 & . & . & . &. \\
\hline $09 / 17 / 2009$ & 5000 & SME & 16282 & process & 5440 & Oxalate & 4904.50385 & . & . & 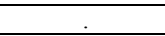 & . \\
\hline $09 / 17 / 2009$ & 5000 & SME & 16282 & process & 5440 & Phosphate & 4904.50385 & & . & - & \\
\hline $09 / 17 / 2009$ & 5000 & $\begin{array}{l}\text { SME } \\
\end{array}$ & 16282 & process & 5457 & Fluoride & 4991.76124 & & . & & \\
\hline $09 / 17 / 2009$ & 5000 & SME & 16282 & process & 5457 & Formate & 4991.76124 & 6.37 & 6.47 & 31784 & 32298 \\
\hline $09 / 17 / 2009$ & 5000 & SME & 16282 & process & 5457 & Chloride & 4991.76124 & & . & 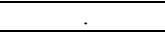 & \\
\hline $09 / 17 / 2009$ & 5000 & SME & 16282 & process & 5457 & Nitrite & 4991.76124 & & & & \\
\hline $09 / 17 / 2009$ & 5000 & $\begin{array}{l}\text { SME } \\
\end{array}$ & 16282 & process & 5457 & Nitrate & 4991.76124 & 3.42 & 3.29 & 17088 & 16440 \\
\hline $09 / 17 / 2009$ & 5000 & SME & 16282 & process & 5457 & Sulfate & 4991.76124 & &. & & \\
\hline $09 / 17 / 2009$ & 5000 & SME & 16282 & process & 5457 & Oxalate & 4991.76124 & & 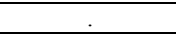 & . & \\
\hline $09 / 17 / 2009$ & 5000 & SME & 16282 & process & 5457 & Phosphate & 4991.76124 & . & . & & \\
\hline $09 / 17 / 2009$ & 5000 & SME & 16282 & process & 5458 & Fluoride & 5112.31772 & & & & \\
\hline $09 / 17 / 2009$ & 5000 & SME & 16282 & process & 5458 & Formate & 5112.31772 & 6.38 & 6.52 & 32620 & 33326 \\
\hline $09 / 17 / 2009$ & 5000 & SME & 16282 & process & 5458 & Chloride & 5112.31772 & & . & & \\
\hline $09 / 17 / 2009$ & 5000 & SME & 16282 & process & 5458 & Nitrite & 5112.31772 & & & & \\
\hline $09 / 17 / 2009$ & 5000 & $\begin{array}{l}\text { SME } \\
\end{array}$ & 16282 & process & 5458 & Nitrate & 5112.31772 & 3.46 & 3.3 & 17702 & 16863 \\
\hline $09 / 17 / 2009$ & 5000 & SME & 16282 & process & 5458 & Sulfate & 5112.31772 & . & . & . & . \\
\hline $09 / 17 / 2009$ & 5000 & SME & 16282 & process & 5458 & Oxalate & 5112.31772 & & . & & \\
\hline $09 / 17 / 2009$ & 5000 & SME & 16282 & process & 5458 & Phosphate & 5112.31772 & &. & & \\
\hline $09 / 17 / 2009$ & 5000 & SME & 16282 & process & 5459 & $\begin{array}{l}\text { Fluoride } \\
\end{array}$ & 5146.52111 & & & & \\
\hline $09 / 17 / 2009$ & 5000 & SME & 16282 & process & 5459 & Formate & 5146.52111 & 6.36 & 6.53 & 32738 & 33586 \\
\hline $09 / 17 / 2009$ & 5000 & SME & 16282 & process & 5459 & Chloride & 5146.52111 & & & & \\
\hline
\end{tabular}


Table A1. Measurements of Standards and SRAT Receipt (SR), SRAT Product (SP), and SME Samples

\begin{tabular}{|c|c|c|c|c|c|c|c|c|c|c|c|}
\hline Date & $\begin{array}{c}\begin{array}{c}\text { Nominal } \\
\text { DF }\end{array} \\
\end{array}$ & Tank & $\begin{array}{c}\text { Sample } \\
\text { Number }\end{array}$ & $\begin{array}{l}\text { Type of } \\
\text { Sample }\end{array}$ & $\begin{array}{c}\text { Bottle } \\
\text { ID }\end{array}$ & Anion & DF & $\begin{array}{c}\text { M-13 ICS3000 } \\
\text { raw (ppm) }\end{array}$ & $\begin{array}{c}\text { M-14 ICS3000 } \\
\text { raw (ppm) }\end{array}$ & $\begin{array}{l}\text { M-13 ICS3000 } \\
\text { FINAL (ppm) } \\
\end{array}$ & $\begin{array}{l}\text { M-14 ICS3000 } \\
\text { FINAL (ppm) } \\
\end{array}$ \\
\hline $09 / 17 / 2009$ & 5000 & SME & 16282 & process & 5459 & Nitrite & 5146.52111 & & & & \\
\hline $09 / 17 / 2009$ & 5000 & SME & 16282 & process & 5459 & Nitrate & 5146.52111 & 3.48 & 3.32 & 17926 & 17104 \\
\hline $09 / 17 / 2009$ & 5000 & SME & 16282 & process & 5459 & Sulfate & 5146.52111 & & - & & - \\
\hline $09 / 17 / 2009$ & 5000 & SME & 16282 & process & 5459 & Oxalate & 5146.52111 & & . & & \\
\hline $09 / 17 / 2009$ & 5000 & SME & 16282 & process & 5459 & Phosphate & 5146.52111 & & & & \\
\hline $09 / 17 / 2009$ & 16 & SME & 16282 & standard & 9999 & Fluoride & 2 & 8.1 & 8.11 & 101.27 & 101.32 \\
\hline $09 / 17 / 2009$ & 16 & SME & 16282 & standard & 9999 & Formate & 2 & 11.46 & 11.49 & 103.46 & 102.85 \\
\hline $09 / 17 / 2009$ & 16 & SME & 16282 & standard & 9999 & Chloride & 2 & 8.1 & 8.03 & 101.27 & 100.33 \\
\hline $09 / 17 / 2009$ & 16 & SME & 16282 & standard & 9999 & Nitrite & 2 & 8.27 & 8.17 & 103.32 & 102.17 \\
\hline $09 / 17 / 2009$ & 16 & SME & 16282 & standard & 9999 & Nitrate & 2 & 10.05 & 9.96 & 103.85 & 103.78 \\
\hline $09 / 17 / 2009$ & 16 & SME & 16282 & standard & 9999 & Sulfate & 2 & 8.18 & 8.09 & 102.23 & 101.19 \\
\hline $09 / 17 / 2009$ & 16 & SME & 16282 & standard & 9999 & Oxalate & 2 & 8.24 & 8.31 & 102.98 & 103.85 \\
\hline $09 / 17 / 2009$ & 16 & SME & 16282 & standard & 9999 & Phosphate & 2 & 7.96 & 7.8 & 99.54 & 97.56 \\
\hline $09 / 17 / 2009$ & 500 & $\begin{array}{l}\text { SME } \\
\end{array}$ & 16282 & process & 5449 & Fluoride & 482.787038 & &. & & \\
\hline $09 / 17 / 2009$ & 500 & SME & 16282 & process & 5449 & Formate & 482.787038 & . & . &. & . \\
\hline $09 / 17 / 2009$ & 500 & SME & 16282 & process & 5449 & Chloride & 482.787038 & . & . & 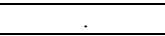 & . \\
\hline $09 / 17 / 2009$ & 500 & SME & 16282 & process & 5449 & Nitrite & 482.787038 & - &. & & \\
\hline $09 / 17 / 2009$ & 500 & SME & 16282 & process & 5449 & Nitrate & 482.787038 & & 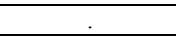 & & \\
\hline $09 / 17 / 2009$ & 500 & SME & 16282 & process & 5449 & Sulfate & 482.787038 & 1.1 & 1.11 & 533 & 536 \\
\hline $09 / 17 / 2009$ & 500 & $\begin{array}{l}\text { SME } \\
\end{array}$ & 16282 & process & 5449 & Oxalate & 482.787038 &. &. &. &. \\
\hline $09 / 17 / 2009$ & 500 & SME & 16282 & process & 5449 & Phosphate & 482.787038 & . & . & . &. \\
\hline $09 / 17 / 2009$ & 500 & SME & 16282 & process & 5450 & Fluoride & 488.261372 &. & . & . & 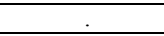 \\
\hline $09 / 17 / 2009$ & 500 & SME & 16282 & process & 5450 & Formate & 488.261372 & & 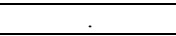 & & \\
\hline $09 / 17 / 2009$ & 500 & $\begin{array}{l}\text { SME } \\
\end{array}$ & 16282 & process & 5450 & $\begin{array}{l}\text { Chloride } \\
\end{array}$ & 488.261372 & . &. & & \\
\hline $09 / 17 / 2009$ & 500 & SME & 16282 & process & 5450 & Nitrite & 488.261372 & & . & & \\
\hline $09 / 17 / 2009$ & 500 & $\begin{array}{l}\text { SME } \\
\end{array}$ & 16282 & process & 5450 & Nitrate & 488.261372 & & & & \\
\hline $09 / 17 / 2009$ & 500 & SME & 16282 & process & 5450 & Sulfate & 488.261372 & 1.08 & 1.07 & 528 & 520 \\
\hline $09 / 17 / 2009$ & 500 & SME & 16282 & process & 5450 & Oxalate & 488.261372 & . & . & . & . \\
\hline $09 / 17 / 2009$ & 500 & SME & 16282 & process & 5450 & Phosphate & 488.261372 & . & . & & 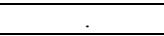 \\
\hline $09 / 17 / 2009$ & 500 & SME & 16282 & process & 5451 & $\begin{array}{l}\text { Fluoride } \\
\end{array}$ & 502.002835 & . & . & . & . \\
\hline $09 / 17 / 2009$ & 500 & SME & 16282 & process & 5451 & Formate & 502.002835 & . & . & . & . \\
\hline $09 / 17 / 2009$ & 500 & SME & 16282 & process & 5451 & Chloride & 502.002835 &. & . & . & 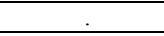 \\
\hline $09 / 17 / 2009$ & 500 & $\begin{array}{l}\text { SME } \\
\end{array}$ & 16282 & process & 5451 & Nitrite & 502.002835 &. & . &. &. \\
\hline $09 / 17 / 2009$ & 500 & SME & 16282 & process & 5451 & Nitrate & 502.002835 & & . & -1 & \\
\hline $09 / 17 / 2009$ & 500 & SME & 16282 & process & 5451 & Sulfate & 502.002835 & 1.09 & 1.06 & 545 & 534 \\
\hline $09 / 17 / 2009$ & 500 & SME & 16282 & process & 5451 & Oxalate & 502.002835 & &. & & \\
\hline $09 / 17 / 2009$ & 500 & SME & 16282 & process & 5451 & Phosphate & 502.002835 & . & . & & $\dot{0}$ \\
\hline $09 / 17 / 2009$ & 500 & SME & 16282 & process & 5452 & Fluoride & 505.857317 & & . & & \\
\hline $09 / 17 / 2009$ & 500 & SME & 16282 & process & 5452 & Formate & 505.857317 &. & . & . & . \\
\hline $09 / 17 / 2009$ & 500 & SME & 16282 & process & 5452 & Chloride & 505.857317 & . & . & . & $\dot{x}$ \\
\hline $09 / 17 / 2009$ & 500 & SME & 16282 & process & 5452 & Nitrite & 505.857317 & - & . & 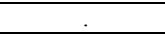 & 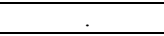 \\
\hline $09 / 17 / 2009$ & 500 & SME & 16282 & process & 5452 & Nitrate & 505.857317 & & & & \\
\hline $09 / 17 / 2009$ & 500 & SME & 16282 & process & 5452 & Sulfate & 505.857317 & 1.09 & 1.12 & 553 & 547 \\
\hline $09 / 17 / 2009$ & 500 & SME & 16282 & process & 5452 & Oxalate & 505.857317 & . & . & & \\
\hline $09 / 17 / 2009$ & 500 & $\begin{array}{l}\text { SME } \\
\end{array}$ & 16282 & process & 5452 & Phosphate & 505.857317 & & & & \\
\hline $09 / 17 / 2009$ & 500 & SR & 16282 & standard & $2 \mathrm{ppm}$ & Fluoride & 1 & 2.03 & 2.04 & 2.03 & 2.04 \\
\hline $09 / 17 / 2009$ & 500 & SR & 16282 & standard & $2 \mathrm{ppm}$ & Formate & 1 & 2.04 & 1.97 & 2.04 & 1.97 \\
\hline $09 / 17 / 2009$ & 500 & SR & 16282 & standard & $2 \mathrm{ppm}$ & Chloride & 1 & 1.98 & 1.89 & 1.98 & 1.89 \\
\hline $09 / 17 / 2009$ & 500 & SR & 16282 & standard & $2 \mathrm{ppm}$ & Nitrite & 1 & 2.04 & 1.95 & 2.04 & 1.95 \\
\hline $09 / 17 / 2009$ & 500 & SR & 16282 & standard & $2 \mathrm{ppm}$ & Nitrate & 1 & 1.95 & 1.9 & 1.95 & 1.9 \\
\hline $09 / 17 / 2009$ & 500 & SR & 16282 & standard & $2 \mathrm{ppm}$ & Sulfate & 1 & 1.99 & 1.95 & 1.99 & 1.95 \\
\hline
\end{tabular}


Table A1. Measurements of Standards and SRAT Receipt (SR), SRAT Product (SP), and SME Samples

\begin{tabular}{|c|c|c|c|c|c|c|c|c|c|c|c|}
\hline Date & $\begin{array}{c}\begin{array}{c}\text { Nominal } \\
\text { DF }\end{array} \\
\end{array}$ & Tank & $\begin{array}{c}\text { Sample } \\
\text { Number }\end{array}$ & $\begin{array}{l}\text { Type of } \\
\text { Sample }\end{array}$ & $\begin{array}{c}\text { Bottle } \\
\text { ID }\end{array}$ & Anion & DF & $\begin{array}{c}\text { M-13 ICS3000 } \\
\text { raw (ppm) }\end{array}$ & $\begin{array}{c}\text { M-14 ICS3000 } \\
\text { raw (ppm) }\end{array}$ & $\begin{array}{l}\text { M-13 ICS3000 } \\
\text { FINAL (ppm) } \\
\end{array}$ & $\begin{array}{l}\text { M-14 ICS3000 } \\
\text { FINAL (ppm) } \\
\end{array}$ \\
\hline $09 / 17 / 2009$ & 500 & SR & 16282 & standard & $2 \mathrm{ppm}$ & Oxalate & 1 & 2.12 & 2.12 & 2.12 & 2.12 \\
\hline $09 / 17 / 2009$ & 500 & SR & 16282 & standard & $2 \mathrm{ppm}$ & Phosphate & 1 & 2.07 & 1.9 & 2.07 & 1.9 \\
\hline $09 / 17 / 2009$ & 500 & SR & 16282 & standard & $16 \mathrm{ppm}$ & Fluoride & 1 & 16.09 & 16.09 & 16.09 & 16.09 \\
\hline $09 / 17 / 2009$ & 500 & SR & 16282 & standard & $16 \mathrm{ppm}$ & Formate & 1 & 16.3 & 16.34 & 16.3 & 16.34 \\
\hline $09 / 17 / 2009$ & 500 & SR & 16282 & standard & $16 \mathrm{ppm}$ & Chloride & 1 & 16.14 & 16.09 & 16.14 & 16.09 \\
\hline $09 / 17 / 2009$ & 500 & SR & 16282 & standard & $16 \mathrm{ppm}$ & Nitrite & 1 & 16.46 & 16.31 & 16.46 & 16.31 \\
\hline $09 / 17 / 2009$ & 500 & SR & 16282 & standard & $16 \mathrm{ppm}$ & Nitrate & 1 & 16.33 & 16.27 & 16.33 & 16.27 \\
\hline $09 / 17 / 2009$ & 500 & SR & 16282 & standard & $16 \mathrm{ppm}$ & Sulfate & 1 & 16.17 & 16.02 & 16.17 & 16.02 \\
\hline $09 / 17 / 2009$ & 500 & SR & 16282 & standard & $16 \mathrm{ppm}$ & Oxalate & 1 & 16.22 & 16.32 & 16.22 & 16.32 \\
\hline $09 / 17 / 2009$ & 500 & SR & 16282 & standard & $16 \mathrm{ppm}$ & Phosphate & 1 & 15.8 & 15.62 & 15.8 & 15.62 \\
\hline $09 / 26 / 2009$ & . & SME & 16384 & standard & $2 \mathrm{ppm}$ & Fluoride & 1 & 2.04 & 2.05 & 2.04 & 2.05 \\
\hline $09 / 26 / 2009$ & . & SME & 16384 & standard & $2 \mathrm{ppm}$ & Formate & 1 & 1.98 & 1.99 & 1.98 & 1.99 \\
\hline $09 / 26 / 2009$ & . & SME & 16384 & standard & $2 \mathrm{ppm}$ & Chloride & 1 & 2.01 & 1.94 & 2.01 & 1.94 \\
\hline $09 / 26 / 2009$ & . & $\begin{array}{l}\text { SME } \\
\end{array}$ & 16384 & standard & $2 \mathrm{ppm}$ & Nitrite & 1 & 2.05 & 2.06 & 2.05 & 2.06 \\
\hline $09 / 26 / 2009$ & . & SME & 16384 & standard & $2 \mathrm{ppm}$ & Nitrate & 1 & 2.03 & 1.93 & 2.03 & 1.93 \\
\hline $09 / 26 / 2009$ & . & SME & 16384 & standard & $2 \mathrm{ppm}$ & Sulfate & 1 & 2.05 & 1.96 & 2.05 & 1.96 \\
\hline $09 / 26 / 2009$ &. & SME & 16384 & standard & $2 \mathrm{ppm}$ & Oxalate & 1 & 2.15 & 2.09 & 2.15 & 2.09 \\
\hline $09 / 26 / 2009$ & . & SME & 16384 & standard & $2 \mathrm{ppm}$ & Phosphate & 1 & 2.05 & 1.98 & 2.05 & 1.98 \\
\hline $09 / 26 / 2009$ & . & SME & 16384 & standard & $16 \mathrm{ppm}$ & $\begin{array}{l}\text { Fluoride } \\
\end{array}$ & 1 & 16.31 & 16.34 & 16.31 & 16.34 \\
\hline $09 / 26 / 2009$ & . & SME & 16384 & standard & $16 \mathrm{ppm}$ & Formate & 1 & 16.17 & 16.26 & 16.17 & 16.26 \\
\hline $09 / 26 / 2009$ & . & SME & 16384 & standard & $16 \mathrm{ppm}$ & Chloride & 1 & 16.3 & 16.26 & 16.3 & 16.26 \\
\hline $09 / 26 / 2009$ & . & SME & 16384 & standard & $16 \mathrm{ppm}$ & Nitrite & 1 & 16.18 & 16.08 & 16.18 & 16.08 \\
\hline $09 / 26 / 2009$ & . & SME & 16384 & standard & $16 \mathrm{ppm}$ & Nitrate & 1 & 16.57 & 16.57 & 16.57 & 16.57 \\
\hline $09 / 26 / 2009$ & . & $\begin{array}{l}\text { SME } \\
\end{array}$ & 16384 & standard & $16 \mathrm{ppm}$ & Sulfate & 1 & 16.47 & 16.22 & 16.47 & 16.22 \\
\hline $09 / 26 / 2009$ & . & SME & 16384 & standard & $16 \mathrm{ppm}$ & Oxalate & 1 & 16.3 & 16.34 & 16.3 & 16.34 \\
\hline $09 / 26 / 2009$ & & $\begin{array}{l}\text { SME } \\
\end{array}$ & 16384 & standard & $16 \mathrm{ppm}$ & Phosphate & 1 & 16.11 & 15.66 & 16.11 & 15.66 \\
\hline $09 / 26 / 2009$ & 5000 & SME & 16384 & process & 5964 & Fluoride & 5082.47633 & & & & \\
\hline $09 / 26 / 2009$ & 5000 & SME & 16384 & process & 5964 & Formate & 5082.47633 & 6.97 & 7.07 & 35426 & 35954 \\
\hline $09 / 26 / 2009$ & 5000 & SME & 16384 & process & 5964 & Chloride & 5082.47633 & 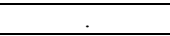 & . & & $\dot{.}$ \\
\hline $09 / 26 / 2009$ & 5000 & SME & 16384 & process & 5964 & Nitrite & 5082.47633 & & - & & \\
\hline $09 / 26 / 2009$ & 5000 & SME & 16384 & process & 5964 & Nitrate & 5082.47633 & 3.81 & 3.68 & 19358 & 18700 \\
\hline $09 / 26 / 2009$ & 5000 & SME & 16384 & process & 5964 & Sulfate & 5082.47633 & & . & $\therefore$ & . \\
\hline $09 / 26 / 2009$ & 5000 & $\begin{array}{l}\text { SME } \\
\end{array}$ & 16384 & process & 5964 & Oxalate & 5082.47633 &. & . &. &. \\
\hline $09 / 26 / 2009$ & 5000 & SME & 16384 & process & 5964 & Phosphate & 5082.47633 & . &. & . & . \\
\hline $09 / 26 / 2009$ & 5000 & SME & 16384 & process & 5965 & Fluoride & 5118.0615 & & & & \\
\hline $09 / 26 / 2009$ & 5000 & SME & 16384 & process & 5965 & Formate & 5118.0615 & 6.97 & 7.25 & 35682 & 37121 \\
\hline $09 / 26 / 2009$ & 5000 & $\begin{array}{l}\text { SME } \\
\end{array}$ & 16384 & process & 5965 & Chloride & 5118.0615 & & . & & 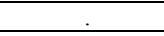 \\
\hline $09 / 26 / 2009$ & 5000 & SME & 16384 & process & 5965 & Nitrite & 5118.0615 & & & & \\
\hline $09 / 26 / 2009$ & 5000 & SME & 16384 & process & 5965 & Nitrate & 5118.0615 & 3.73 & 3.45 & 19088 & 17655 \\
\hline $09 / 26 / 2009$ & 5000 & SME & 16384 & process & 5965 & Sulfate & 5118.0615 & $\therefore$ & . & $\therefore$ & . \\
\hline $09 / 26 / 2009$ & 5000 & SME & 16384 & process & 5965 & Oxalate & 5118.0615 & - & . &. & 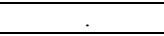 \\
\hline $09 / 26 / 2009$ & 5000 & SME & 16384 & process & 5965 & Phosphate & 5118.0615 & &. & & \\
\hline $09 / 26 / 2009$ & 5000 & SME & 16384 & process & 5966 & Fluoride & 5028.78687 & & & & \\
\hline $09 / 26 / 2009$ & 5000 & SME & 16384 & process & 5966 & Formate & 5028.78687 & 7.1 & 7.24 & 35715 & 36387 \\
\hline $09 / 26 / 2009$ & 5000 & $\begin{array}{l}\text { SME } \\
\end{array}$ & 16384 & process & 5966 & Chloride & 5028.78687 & & . & & \\
\hline $09 / 26 / 2009$ & 5000 & SME & 16384 & process & 5966 & Nitrite & 5028.78687 & & . & & \\
\hline $09 / 26 / 2009$ & 5000 & SME & 16384 & process & 5966 & Nitrate & 5028.78687 & 3.8 & 3.7 & 19123 & 18603 \\
\hline $09 / 26 / 2009$ & 5000 & SME & 16384 & process & 5966 & Sulfate & 5028.78687 & & $\therefore$ & & \\
\hline $09 / 26 / 2009$ & 5000 & SME & 16384 & process & 5966 & Oxalate & 5028.78687 & &. & & \\
\hline $09 / 26 / 2009$ & 5000 & SME & 16384 & process & 5966 & Phosphate & 5028.78687 & & . & & \\
\hline $09 / 26 / 2009$ & 5000 & SME & 16384 & process & 5967 & Fluoride & 4915.39841 & & & & \\
\hline
\end{tabular}


Table A1. Measurements of Standards and SRAT Receipt (SR), SRAT Product (SP), and SME Samples

\begin{tabular}{|c|c|c|c|c|c|c|c|c|c|c|c|}
\hline Date & $\begin{array}{c}\begin{array}{c}\text { Nominal } \\
\text { DF }\end{array} \\
\end{array}$ & Tank & $\begin{array}{c}\text { Sample } \\
\text { Number }\end{array}$ & $\begin{array}{l}\text { Type of } \\
\text { Sample }\end{array}$ & $\begin{array}{c}\text { Bottle } \\
\text { ID }\end{array}$ & Anion & DF & $\begin{array}{c}\text { M-13 ICS3000 } \\
\text { raw (ppm) }\end{array}$ & $\begin{array}{c}\text { M-14 ICS3000 } \\
\text { raw (ppm) }\end{array}$ & $\begin{array}{l}\text { M-13 ICS3000 } \\
\text { FINAL (ppm) } \\
\end{array}$ & $\begin{array}{l}\text { M-14 ICS3000 } \\
\text { FINAL (ppm) } \\
\end{array}$ \\
\hline $09 / 26 / 2009$ & 5000 & SME & 16384 & process & 5967 & Formate & 4915.39841 & 6.89 & 7.02 & 33886 & 34517 \\
\hline $09 / 26 / 2009$ & 5000 & SME & 16384 & process & 5967 & Chloride & 4915.39841 & & . & - & - \\
\hline $09 / 26 / 2009$ & 5000 & SME & 16384 & process & 5967 & Nitrite & 4915.39841 & & & & \\
\hline $09 / 26 / 2009$ & 5000 & SME & 16384 & process & 5967 & Nitrate & 4915.39841 & 3.69 & 3.62 & 18136 & 17816 \\
\hline $09 / 26 / 2009$ & 5000 & SME & 16384 & process & 5967 & Sulfate & 4915.39841 & . & . & & - \\
\hline $09 / 26 / 2009$ & 5000 & SME & 16384 & process & 5967 & Oxalate & 4915.39841 & & . & & \\
\hline $09 / 26 / 2009$ & 5000 & SME & 16384 & process & 5967 & Phosphate & 4915.39841 & & & & \\
\hline $09 / 26 / 2009$ & 16 & SME & 16384 & standard & 9999 & Fluoride & 2 & 8.23 & 8.24 & 102.85 & 102.99 \\
\hline $09 / 26 / 2009$ & 16 & SME & 16384 & standard & 9999 & Formate & 2 & 11.58 & 11.8 & 101.71 & 103.67 \\
\hline $09 / 26 / 2009$ & 16 & SME & 16384 & standard & 9999 & Chloride & 2 & 8.2 & 8.14 & 102.46 & 101.71 \\
\hline $09 / 26 / 2009$ & 16 & SME & 16384 & standard & 9999 & Nitrite & 2 & 8.13 & 8.11 & 101.62 & 101.34 \\
\hline $09 / 26 / 2009$ & 16 & SME & 16384 & standard & 9999 & Nitrate & 2 & 10.43 & 10.27 & 107.28 & 105.77 \\
\hline $09 / 26 / 2009$ & 16 & SME & 16384 & standard & 9999 & Sulfate & 2 & 8.37 & 8.19 & 104.62 & 102.39 \\
\hline $09 / 26 / 2009$ & 16 & SME & 16384 & standard & 9999 & Oxalate & 2 & 8.37 & 8.37 & 104.67 & 104.65 \\
\hline $09 / 26 / 2009$ & 16 & SME & 16384 & standard & 9999 & Phosphate & 2 & 8.17 & 7.86 & 102.16 & 98.29 \\
\hline $09 / 26 / 2009$ & 500 & SME & 16384 & process & 5972 & $\begin{array}{l}\text { Fluoride } \\
\end{array}$ & 499.172453 & . & . & . & . \\
\hline $09 / 26 / 2009$ & 500 & SME & 16384 & process & 5972 & Formate & 499.172453 & - &. & & \\
\hline $09 / 26 / 2009$ & 500 & SME & 16384 & process & 5972 & Chloride & 499.172453 & . & . & . & . \\
\hline $09 / 26 / 2009$ & 500 & SME & 16384 & process & 5972 & Nitrite & 499.172453 & & . & & \\
\hline $09 / 26 / 2009$ & 500 & SME & 16384 & process & 5972 & Nitrate & 499.172453 & & - & & \\
\hline $09 / 26 / 2009$ & 500 & SME & 16384 & process & 5972 & Sulfate & 499.172453 & 1.24 & 1.19 & 617 & 592 \\
\hline $09 / 26 / 2009$ & 500 & SME & 16384 & process & 5972 & Oxalate & 499.172453 &. & . & . & . \\
\hline $09 / 26 / 2009$ & 500 & SME & 16384 & process & 5972 & Phosphate & 499.172453 & & - & & \\
\hline $09 / 26 / 2009$ & 500 & SME & 16384 & process & 5973 & Fluoride & 508.475917 & . &. & & \\
\hline $09 / 26 / 2009$ & 500 & SME & 16384 & process & 5973 & Formate & 508.475917 & & . & & \\
\hline $09 / 26 / 2009$ & 500 & SME & 16384 & process & 5973 & $\begin{array}{l}\text { Chloride } \\
\end{array}$ & 508.475917 & . &. & & \\
\hline $09 / 26 / 2009$ & 500 & SME & 16384 & process & 5973 & Nitrite & 508.475917 & . & . &. & . \\
\hline $09 / 26 / 2009$ & 500 & SME & 16384 & process & 5973 & Nitrate & 508.475917 & & & & \\
\hline $09 / 26 / 2009$ & 500 & SME & 16384 & process & 5973 & Sulfate & 508.475917 & 1.2 & 1.15 & 608 & 586 \\
\hline $09 / 26 / 2009$ & 500 & SME & 16384 & process & 5973 & Oxalate & 508.475917 & . & . & 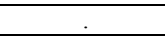 & . \\
\hline $09 / 26 / 2009$ & 500 & SME & 16384 & process & 5973 & Phosphate & 508.475917 & . & . & . & . \\
\hline $09 / 26 / 2009$ & 500 & SME & 16384 & process & 5974 & Fluoride & 492.102594 &. & . & 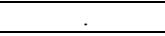 & 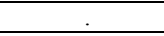 \\
\hline $09 / 26 / 2009$ & 500 & SME & 16384 & process & 5974 & Formate & 492.102594 &. & . &. &. \\
\hline $09 / 26 / 2009$ & 500 & SME & 16384 & process & 5974 & Chloride & 492.102594 & . &. & . & . \\
\hline $09 / 26 / 2009$ & 500 & SME & 16384 & process & 5974 & Nitrite & 492.102594 & &. & & \\
\hline $09 / 26 / 2009$ & 500 & SME & 16384 & process & 5974 & Nitrate & 492.102594 & & & & \\
\hline $09 / 26 / 2009$ & 500 & SME & 16384 & process & 5974 & Sulfate & 492.102594 & 1.22 & 1.17 & 602 & 575 \\
\hline $09 / 26 / 2009$ & 500 & SME & 16384 & process & 5974 & Oxalate & 492.102594 & & . & & \\
\hline $09 / 26 / 2009$ & 500 & SME & 16384 & process & 5974 & Phosphate & 492.102594 &. & . & $\dot{\square}$ & \\
\hline $09 / 26 / 2009$ & 500 & SME & 16384 & process & 5975 & Fluoride & 488.53405 & . & . & . & . \\
\hline $09 / 26 / 2009$ & 500 & SME & 16384 & process & 5975 & Formate & 488.53405 &. & . &. & . \\
\hline $09 / 26 / 2009$ & 500 & SME & 16384 & process & 5975 & Chloride & 488.53405 & $\dot{\square}$ &. & & $\dot{\square}$ \\
\hline $09 / 26 / 2009$ & 500 & SME & 16384 & process & 5975 & Nitrite & 488.53405 & 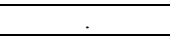 & . & & \\
\hline $09 / 26 / 2009$ & 500 & SME & 16384 & process & 5975 & Nitrate & 488.53405 & & & & \\
\hline $09 / 26 / 2009$ & 500 & SME & 16384 & process & 5975 & Sulfate & 488.53405 & 1.24 & 1.18 & 607 & 576 \\
\hline $09 / 26 / 2009$ & 500 & SME & 16384 & process & 5975 & Oxalate & 488.53405 & . & . & . & . \\
\hline $09 / 26 / 2009$ & 500 & SME & 16384 & process & 5975 & Phosphate & 488.53405 & & & & \\
\hline $09 / 26 / 2009$ & 500 & SR & 16384 & standard & $2 \mathrm{ppm}$ & Fluoride & 1 & 2.04 & 2.04 & 2.04 & 2.04 \\
\hline $09 / 26 / 2009$ & 500 & SR & 16384 & standard & $2 \mathrm{ppm}$ & Formate & 1 & 1.97 & 2.02 & 1.97 & 2.02 \\
\hline $09 / 26 / 2009$ & 500 & SR & 16384 & standard & $2 \mathrm{ppm}$ & Chloride & 1 & 2 & 1.93 & 2 & 1.93 \\
\hline $09 / 26 / 2009$ & 500 & SR & 16384 & standard & $2 \mathrm{ppm}$ & Nitrite & 1 & 2.04 & 2.05 & 2.04 & 2.05 \\
\hline
\end{tabular}


Table A1. Measurements of Standards and SRAT Receipt (SR), SRAT Product (SP), and SME Samples

\begin{tabular}{|c|c|c|c|c|c|c|c|c|c|c|c|}
\hline Date & $\begin{array}{c}\text { Nominal } \\
\text { DF }\end{array}$ & Tank & $\begin{array}{c}\text { Sample } \\
\text { Number }\end{array}$ & $\begin{array}{l}\text { Type of } \\
\text { Sample }\end{array}$ & $\begin{array}{c}\text { Bottle } \\
\text { ID }\end{array}$ & Anion & DF & $\begin{array}{l}\text { M-13 ICS3000 } \\
\text { raw (ppm) }\end{array}$ & $\begin{array}{l}\text { M-14 ICS3000 } \\
\text { raw (ppm) }\end{array}$ & $\begin{array}{l}\text { M-13 ICS3000 } \\
\text { FINAL (ppm) }\end{array}$ & $\begin{array}{l}\text { M-14 ICS3000 } \\
\text { FINAL (ppm) }\end{array}$ \\
\hline $09 / 26 / 2009$ & 500 & SR & 16384 & standard & $2 \mathrm{ppm}$ & Nitrate & 1 & 2.02 & 1.97 & 2.02 & 1.97 \\
\hline $09 / 26 / 2009$ & 500 & SR & 16384 & standard & $2 \mathrm{ppm}$ & Sulfate & 1 & 2.03 & 1.96 & 2.03 & 1.96 \\
\hline $09 / 26 / 2009$ & 500 & SR & 16384 & standard & $2 \mathrm{ppm}$ & Oxalate & 1 & 2.13 & 2.09 & 2.13 & 2.09 \\
\hline $09 / 26 / 2009$ & 500 & SR & 16384 & standard & $2 \mathrm{ppm}$ & Phosphate & 1 & 1.97 & 1.96 & 1.97 & 1.96 \\
\hline $09 / 26 / 2009$ & 500 & SR & 16384 & standard & $16 \mathrm{ppm}$ & Fluoride & 1 & 16.32 & 16.36 & 16.32 & 16.36 \\
\hline $09 / 26 / 2009$ & 500 & SR & 16384 & standard & $16 \mathrm{ppm}$ & Formate & 1 & 16.26 & 16.09 & 16.26 & 16.09 \\
\hline $09 / 26 / 2009$ & 500 & SR & 16384 & standard & $16 \mathrm{ppm}$ & Chloride & 1 & 16.29 & 16.32 & 16.29 & 16.32 \\
\hline $09 / 26 / 2009$ & 500 & SR & 16384 & standard & $16 \mathrm{ppm}$ & Nitrite & 1 & 16.17 & 16.23 & 16.17 & 16.23 \\
\hline $09 / 26 / 2009$ & 500 & SR & 16384 & standard & $16 \mathrm{ppm}$ & Nitrate & 1 & 16.53 & 16.59 & 16.53 & 16.59 \\
\hline $09 / 26 / 2009$ & 500 & SR & 16384 & standard & $16 \mathrm{ppm}$ & Sulfate & 1 & 16.41 & 16.28 & 16.41 & 16.28 \\
\hline $09 / 26 / 2009$ & 500 & SR & 16384 & standard & $16 \mathrm{ppm}$ & Oxalate & 1 & 16.33 & 16.5 & 16.33 & 16.5 \\
\hline $09 / 26 / 2009$ & 500 & SR & 16384 & standard & $16 \mathrm{ppm}$ & Phosphate & 1 & 15.96 & 15.74 & 15.96 & 15.74 \\
\hline
\end{tabular}


Table A2. Measurements of Simulants of SRAT Receipt, SRAT Product, and SME, of Standards Used for Calibration, and of Standards from Developed from Dried Salts

\begin{tabular}{|c|c|c|c|c|c|c|}
\hline Type & Simulant & \begin{tabular}{|c|} 
Known \\
Concentration (ppm) \\
\end{tabular} & Anion & Date & $\begin{array}{c}\text { M-14 ICS 3000 } \\
\text { Measurement (ppm) } \\
\end{array}$ & $\begin{array}{c}\text { M-13 ICS 3000 } \\
\text { Measurement (ppm) }\end{array}$ \\
\hline Simulated Sample Crosscheck Data 500X Dilution & SME & 1 & Fluoride & $9 / 15$ & 1.03 & 1.02 \\
\hline Simulated Sample Crosscheck Data 500X Dilution & SME & 60 & Formate & $9 / 15$ & 56.31 & 55.48 \\
\hline Simulated Sample Crosscheck Data 500X Dilution & SME & 1 & Chloride & $9 / 15$ & 0.97 & 1.01 \\
\hline Simulated Sample Crosscheck Data 500X Dilution & SME & 1 & Nitrite & $9 / 15$ & 0.99 & 1.02 \\
\hline Simulated Sample Crosscheck Data 500X Dilution & SME & 25 & Nitrate & $9 / 15$ & 24.89 & 25.14 \\
\hline Simulated Sample Crosscheck Data 500X Dilution & SME & 2.5 & Sulfate & $9 / 15$ & 2.49 & 2.55 \\
\hline Simulated Sample Crosscheck Data 500X Dilution & SME & 2.5 & Oxalate & $9 / 15$ & 2.67 & 2.71 \\
\hline Simulated Sample Crosscheck Data 500X Dilution & SME & 2.5 & Phosphate & $9 / 15$ & 2.47 & 2.71 \\
\hline Simulated Sample Crosscheck Data 500X Dilution & SME & 1 & Fluoride & $9 / 15$ & 1.02 & 1.03 \\
\hline Simulated Sample Crosscheck Data 500X Dilution & SME & 60 & Formate & $9 / 15$ & 56.27 & 55.5 \\
\hline Simulated Sample Crosscheck Data 500X Dilution & SME & 1 & Chloride & $9 / 15$ & 0.97 & 1.01 \\
\hline Simulated Sample Crosscheck Data 500X Dilution & SME & 1 & Nitrite & $9 / 15$ & 0.99 & 1.02 \\
\hline Simulated Sample Crosscheck Data 500X Dilution & SME & 25 & Nitrate & $9 / 15$ & 24.86 & 25.15 \\
\hline Simulated Sample Crosscheck Data 500X Dilution & SME & 2.5 & Sulfate & $9 / 15$ & 2.49 & 2.71 \\
\hline Simulated Sample Crosscheck Data 500X Dilution & SME & 2.5 & Oxalate & $9 / 15$ & 2.67 & 2.63 \\
\hline Simulated Sample Crosscheck Data 500X Dilution & SME & 2.5 & Phosphate & $9 / 15$ & 2.47 & 2.71 \\
\hline Simulated Sample Crosscheck Data 500X Dilution & SME & 1 & Fluoride & $9 / 15$ & 1.02 & 1.03 \\
\hline Simulated Sample Crosscheck Data 500X Dilution & SME & 60 & Formate & $9 / 15$ & 56.38 & 55.52 \\
\hline Simulated Sample Crosscheck Data 500X Dilution & SME & 1 & Chloride & $9 / 15$ & 0.97 & 1.01 \\
\hline Simulated Sample Crosscheck Data 500X Dilution & SME & 1 & Nitrite & $9 / 15$ & 0.99 & 1.02 \\
\hline Simulated Sample Crosscheck Data 500X Dilution & SME & 25 & Nitrate & $9 / 15$ & 24.92 & 25.16 \\
\hline Simulated Sample Crosscheck Data 500X Dilution & SME & 2.5 & Sulfate & $9 / 15$ & 2.49 & 2.55 \\
\hline Simulated Sample Crosscheck Data 500X Dilution & SME & 2.5 & Oxalate & $9 / 15$ & 2.67 & 2.71 \\
\hline Simulated Sample Crosscheck Data 500X Dilution & SME & 2.5 & Phosphate & $9 / 15$ & 2.48 & 2.63 \\
\hline Simulated Sample Crosscheck Data 500X Dilution & SME & 1 & Fluoride & $9 / 16$ & 1.03 & 1.02 \\
\hline Simulated Sample Crosscheck Data 500X Dilution & SME & 60 & Formate & $9 / 16$ & 56.36 & 53.83 \\
\hline Simulated Sample Crosscheck Data 500X Dilution & SME & 1 & Chloride & $9 / 16$ & 0.96 & 1.01 \\
\hline Simulated Sample Crosscheck Data 500X Dilution & SME & 1 & Nitrite & $9 / 16$ & 0.99 & 1.03 \\
\hline Simulated Sample Crosscheck Data 500X Dilution & SME & 25 & Nitrate & $9 / 16$ & 24.85 & 24.98 \\
\hline Simulated Sample Crosscheck Data 500X Dilution & SME & 2.5 & Sulfate & $9 / 16$ & 2.48 & 2.5 \\
\hline Simulated Sample Crosscheck Data 500X Dilution & SME & 2.5 & Oxalate & $9 / 16$ & 2.67 & 2.66 \\
\hline Simulated Sample Crosscheck Data 500X Dilution & SME & 2.5 & Phosphate & $9 / 16$ & 2.47 & 2.54 \\
\hline Simulated Sample Crosscheck Data 500X Dilution & SME & 1 & Fluoride & $9 / 16$ & 1.03 & 1.02 \\
\hline Simulated Sample Crosscheck Data 500X Dilution & SME & 60 & Formate & $9 / 16$ & 56.38 & 53.86 \\
\hline Simulated Sample Crosscheck Data 500X Dilution & SME & 1 & Chloride & $9 / 16$ & 0.96 & 1.01 \\
\hline Simulated Sample Crosscheck Data 500X Dilution & SME & 1 & Nitrite & $9 / 16$ & 0.98 & 1.03 \\
\hline Simulated Sample Crosscheck Data 500X Dilution & SME & 25 & Nitrate & $9 / 16$ & 24.87 & 25.01 \\
\hline Simulated Sample Crosscheck Data 500X Dilution & SME & 2.5 & Sulfate & $9 / 16$ & 2.48 & 2.5 \\
\hline Simulated Sample Crosscheck Data 500X Dilution & SME & 2.5 & Oxalate & $9 / 16$ & 2.67 & 2.66 \\
\hline Simulated Sample Crosscheck Data 500X Dilution & SME & 2.5 & Phosphate & $9 / 16$ & 2.47 & 2.55 \\
\hline Simulated Sample Crosscheck Data 500X Dilution & SRAT Receipt & 1 & Fluoride & $9 / 15$ & 1.02 & 1.02 \\
\hline Simulated Sample Crosscheck Data 500X Dilution & SRAT Receipt & 15 & Formate & $9 / 15$ & 15.65 & 15.54 \\
\hline Simulated Sample Crosscheck Data 500X Dilution & SRAT Receipt & 1 & Chloride & $9 / 15$ & 0.98 & 1 \\
\hline Simulated Sample Crosscheck Data 500X Dilution & SRAT Receipt & 15 & Nitrite & $9 / 15$ & 15.63 & 15.11 \\
\hline Simulated Sample Crosscheck Data 500X Dilution & SRAT Receipt & 15 & Nitrate & $9 / 15$ & 15.04 & 15.09 \\
\hline Simulated Sample Crosscheck Data 500X Dilution & SRAT Receipt & 2.5 & Sulfate & $9 / 15$ & 2.47 & 2.53 \\
\hline Simulated Sample Crosscheck Data 500X Dilution & SRAT Receipt & 2.5 & Oxalate & $9 / 15$ & 2.67 & 2.71 \\
\hline Simulated Sample Crosscheck Data 500X Dilution & SRAT Receipt & 2.5 & Phosphate & $9 / 15$ & 2.48 & 2.64 \\
\hline Simulated Sample Crosscheck Data 500X Dilution & SRAT Receipt & 1 & Fluoride & $9 / 15$ & 1.02 & 1.02 \\
\hline Simulated Sample Crosscheck Data 500X Dilution & SRAT Receipt & 15 & Formate & $9 / 15$ & 15.56 & 15.56 \\
\hline
\end{tabular}


Table A2. Measurements of Simulants of SRAT Receipt, SRAT Product, and SME, of Standards Used for Calibration, and of Standards from Developed from Dried Salts

\begin{tabular}{|c|c|c|c|c|c|c|}
\hline Type & Simulant & \begin{tabular}{|c|} 
Known \\
Concentration (ppm)
\end{tabular} & Anion & Date & $\begin{array}{c}\text { M-14 ICS 3000 } \\
\text { Measurement (ppm) }\end{array}$ & $\begin{array}{c}\text { M-13 ICS 3000 } \\
\text { Measurement (ppm) }\end{array}$ \\
\hline Simulated Sample Crosscheck Data 500X Dilution & SRAT Receipt & 1 & Chloride & $9 / 15$ & 1.03 & 1.03 \\
\hline Simulated Sample Crosscheck Data 500X Dilution & SRAT Receipt & 15 & Nitrite & $9 / 15$ & 15.65 & 15.11 \\
\hline Simulated Sample Crosscheck Data 500X Dilution & SRAT Receipt & 15 & Nitrate & $9 / 15$ & 15.03 & 15.1 \\
\hline Simulated Sample Crosscheck Data 500X Dilution & SRAT Receipt & 2.5 & Sulfate & $9 / 15$ & 2.47 & 2.54 \\
\hline Simulated Sample Crosscheck Data 500X Dilution & SRAT Receipt & 2.5 & Oxalate & $9 / 15$ & 2.67 & 2.71 \\
\hline Simulated Sample Crosscheck Data 500X Dilution & SRAT Receipt & 2.5 & Phosphate & $9 / 15$ & 2.48 & 2.63 \\
\hline Simulated Sample Crosscheck Data 500X Dilution & SRAT Receipt & 1 & Fluoride & $9 / 15$ & 1.02 & 1.02 \\
\hline Simulated Sample Crosscheck Data 500X Dilution & SRAT Receipt & 15 & Formate & $9 / 15$ & 15.54 & 15.54 \\
\hline Simulated Sample Crosscheck Data 500X Dilution & SRAT Receipt & 1 & Chloride & $9 / 15$ & 0.98 & 1 \\
\hline Simulated Sample Crosscheck Data 500X Dilution & SRAT Receipt & 15 & Nitrite & $9 / 15$ & 15.65 & 15.1 \\
\hline Simulated Sample Crosscheck Data 500X Dilution & SRAT Receipt & 15 & Nitrate & $9 / 15$ & 15.02 & 15.08 \\
\hline Simulated Sample Crosscheck Data 500X Dilution & SRAT Receipt & 2.5 & Sulfate & $9 / 15$ & 2.47 & 2.53 \\
\hline Simulated Sample Crosscheck Data 500X Dilution & SRAT Receipt & 2.5 & Oxalate & $9 / 15$ & 2.67 & 2.71 \\
\hline Simulated Sample Crosscheck Data 500X Dilution & SRAT Receipt & 2.5 & Phosphate & $9 / 15$ & 2.47 & 2.64 \\
\hline Simulated Sample Crosscheck Data 500X Dilution & SRAT Receipt & 1 & Fluoride & $9 / 16$ & 1.02 & 1.01 \\
\hline Simulated Sample Crosscheck Data 500X Dilution & SRAT Receipt & 15 & Formate & $9 / 16$ & 15.59 & 14.75 \\
\hline Simulated Sample Crosscheck Data 500X Dilution & SRAT Receipt & 1 & Chloride & $9 / 16$ & 0.97 & 0.99 \\
\hline Simulated Sample Crosscheck Data 500X Dilution & SRAT Receipt & 15 & Nitrite & $9 / 16$ & 15.58 & 15.15 \\
\hline Simulated Sample Crosscheck Data 500X Dilution & SRAT Receipt & 15 & Nitrate & $9 / 16$ & 15 & 15.01 \\
\hline Simulated Sample Crosscheck Data 500X Dilution & SRAT Receipt & 2.5 & Sulfate & $9 / 16$ & 2.46 & 2.49 \\
\hline Simulated Sample Crosscheck Data 500X Dilution & SRAT Receipt & 2.5 & Oxalate & $9 / 16$ & 2.67 & 2.67 \\
\hline Simulated Sample Crosscheck Data 500X Dilution & SRAT Receipt & 2.5 & Phosphate & $9 / 16$ & 2.47 & 2.56 \\
\hline Simulated Sample Crosscheck Data 500X Dilution & SRAT Receipt & 1 & Fluoride & $9 / 16$ & 1.02 & 1.01 \\
\hline Simulated Sample Crosscheck Data 500X Dilution & SRAT Receipt & 15 & Formate & $9 / 16$ & 15.55 & 14.75 \\
\hline Simulated Sample Crosscheck Data 500X Dilution & SRAT Receipt & 1 & Chloride & $9 / 16$ & 0.97 & 0.99 \\
\hline Simulated Sample Crosscheck Data 500X Dilution & SRAT Receipt & 15 & Nitrite & $9 / 16$ & 15.59 & 15.15 \\
\hline Simulated Sample Crosscheck Data 500X Dilution & SRAT Receipt & 15 & Nitrate & $9 / 16$ & 14.98 & 15.02 \\
\hline Simulated Sample Crosscheck Data 500X Dilution & SRAT Receipt & 2.5 & Sulfate & $9 / 16$ & 2.46 & 2.49 \\
\hline Simulated Sample Crosscheck Data 500X Dilution & SRAT Receipt & 2.5 & Oxalate & $9 / 16$ & 2.67 & 2.68 \\
\hline Simulated Sample Crosscheck Data 500X Dilution & SRAT Receipt & 2.5 & Phosphate & $9 / 16$ & 2.47 & 2.57 \\
\hline Simulated Sample Crosscheck Data 5000X Dilution & SME & 1 & Fluoride & $9 / 15$ & 1.03 & 1.02 \\
\hline Simulated Sample Crosscheck Data 5000X Dilution & SME & 6 & Formate & $9 / 15$ & 6.15 & 6.47 \\
\hline Simulated Sample Crosscheck Data 5000X Dilution & SME & 1 & Chloride & $9 / 15$ & 1.03 & 1.01 \\
\hline Simulated Sample Crosscheck Data 5000X Dilution & SME & 1 & Nitrite & $9 / 15$ & 1 & 1.01 \\
\hline Simulated Sample Crosscheck Data 5000X Dilution & SME & 2.5 & Nitrate & $9 / 15$ & 2.5 & 2.56 \\
\hline Simulated Sample Crosscheck Data 5000X Dilution & SME & 1 & Sulfate & $9 / 15$ & 1.02 & 1.03 \\
\hline Simulated Sample Crosscheck Data 5000X Dilution & SME & 1 & Oxalate & $9 / 15$ & 1.1 & 1.09 \\
\hline Simulated Sample Crosscheck Data 5000X Dilution & SME & 1 & Phosphate & $9 / 15$ & 1.01 & 1.06 \\
\hline Simulated Sample Crosscheck Data 5000X Dilution & SME & 1 & Fluoride & $9 / 15$ & 1.02 & 1.02 \\
\hline Simulated Sample Crosscheck Data 5000X Dilution & SME & 6 & Formate & $9 / 15$ & 6.13 & 6.47 \\
\hline Simulated Sample Crosscheck Data 5000X Dilution & SME & 1 & Chloride & $9 / 15$ & 0.97 & 1.01 \\
\hline Simulated Sample Crosscheck Data 5000X Dilution & SME & 1 & Nitrite & $9 / 15$ & 0.98 & 1.01 \\
\hline Simulated Sample Crosscheck Data 5000X Dilution & SME & 2.5 & Nitrate & $9 / 15$ & 2.47 & 2.56 \\
\hline Simulated Sample Crosscheck Data 5000X Dilution & SME & 1 & Sulfate & $9 / 15$ & 1 & 1.03 \\
\hline Simulated Sample Crosscheck Data 5000X Dilution & SME & 1 & Oxalate & $9 / 15$ & 1.07 & 1.1 \\
\hline Simulated Sample Crosscheck Data 5000X Dilution & SME & 1 & Phosphate & $9 / 15$ & 0.99 & 1.07 \\
\hline Simulated Sample Crosscheck Data 5000X Dilution & SME & 1 & Fluoride & $9 / 15$ & 1.02 & 1.02 \\
\hline Simulated Sample Crosscheck Data 5000X Dilution & SME & 6 & Formate & $9 / 15$ & 6.15 & 6.46 \\
\hline Simulated Sample Crosscheck Data 5000X Dilution & SME & 1 & Chloride & $9 / 15$ & 0.97 & 1.01 \\
\hline Simulated Sample Crosscheck Data 5000X Dilution & SME & 1 & Nitrite & $9 / 15$ & 0.98 & 1.01 \\
\hline
\end{tabular}


Table A2. Measurements of Simulants of SRAT Receipt, SRAT Product, and SME, of Standards Used for Calibration, and of Standards from Developed from Dried Salts

\begin{tabular}{|c|c|c|c|c|c|c|}
\hline Type & Simulant & \begin{tabular}{|c|} 
Known \\
Concentration (ppm) \\
\end{tabular} & Anion & Date & $\begin{array}{c}\text { M-14 ICS 3000 } \\
\text { Measurement (ppm) } \\
\end{array}$ & $\begin{array}{c}\text { M-13 ICS 3000 } \\
\text { Measurement (ppm) }\end{array}$ \\
\hline Simulated Sample Crosscheck Data 5000X Dilution & SME & 2.5 & Nitrate & $9 / 15$ & 2.47 & 2.56 \\
\hline Simulated Sample Crosscheck Data 5000X Dilution & SME & 1 & Sulfate & $9 / 15$ & 0.99 & 1.03 \\
\hline Simulated Sample Crosscheck Data 5000X Dilution & SME & 1 & Oxalate & $9 / 15$ & 1.07 & 1.09 \\
\hline Simulated Sample Crosscheck Data 5000X Dilution & SME & 1 & Phosphate & $9 / 15$ & 0.98 & 1.07 \\
\hline Simulated Sample Crosscheck Data 5000X Dilution & SME & 1 & Fluoride & $9 / 16$ & 1.03 & 1.02 \\
\hline Simulated Sample Crosscheck Data 5000X Dilution & SME & 6 & Formate & $9 / 16$ & 6.14 & 5.99 \\
\hline Simulated Sample Crosscheck Data 5000X Dilution & SME & 1 & Chloride & $9 / 16$ & 0.97 & 1 \\
\hline Simulated Sample Crosscheck Data 5000X Dilution & SME & 1 & Nitrite & $9 / 16$ & 0.98 & 1 \\
\hline Simulated Sample Crosscheck Data 5000X Dilution & SME & 2.5 & Nitrate & $9 / 16$ & 2.46 & 2.52 \\
\hline Simulated Sample Crosscheck Data 5000X Dilution & SME & 1 & Sulfate & $9 / 16$ & 1 & 0.98 \\
\hline Simulated Sample Crosscheck Data 5000X Dilution & SME & 1 & Oxalate & $9 / 16$ & 1.08 & 1.03 \\
\hline Simulated Sample Crosscheck Data 5000X Dilution & SME & 1 & Phosphate & $9 / 16$ & 0.99 & 1.02 \\
\hline Simulated Sample Crosscheck Data 5000X Dilution & SME & 1 & Fluoride & $9 / 16$ & 1.02 & 1.02 \\
\hline $\begin{array}{l}\text { Simulated Sample Crosscheck Data 5000X Dilution } \\
\end{array}$ & SME & 6 & Formate & $9 / 16$ & 6.15 & 5.99 \\
\hline Simulated Sample Crosscheck Data 5000X Dilution & SME & 1 & Chloride & $9 / 16$ & 0.96 & 1 \\
\hline Simulated Sample Crosscheck Data 5000X Dilution & SME & 1 & Nitrite & $9 / 16$ & 0.97 & 1 \\
\hline Simulated Sample Crosscheck Data 5000X Dilution & SME & 2.5 & Nitrate & $9 / 16$ & 2.44 & 2.53 \\
\hline Simulated Sample Crosscheck Data 5000X Dilution & SME & 1 & Sulfate & $9 / 16$ & 0.99 & 0.98 \\
\hline Simulated Sample Crosscheck Data 5000X Dilution & SME & 1 & Oxalate & $9 / 16$ & 1.06 & 1.05 \\
\hline Simulated Sample Crosscheck Data 5000X Dilution & SME & 1 & Phosphate & $9 / 16$ & 0.97 & 1.03 \\
\hline Simulated Sample Crosscheck Data 5000X Dilution & SRAT Receipt & 1 & Fluoride & $9 / 15$ & 1.02 & 1.02 \\
\hline Simulated Sample Crosscheck Data 5000X Dilution & SRAT Receipt & 1.5 & Formate & $9 / 15$ & 1.52 & 1.52 \\
\hline Simulated Sample Crosscheck Data 5000X Dilution & SRAT Receipt & 1 & Chloride & $9 / 15$ & 0.96 & 1 \\
\hline Simulated Sample Crosscheck Data 5000X Dilution & SRAT Receipt & 1.5 & Nitrite & $9 / 15$ & 1.49 & 1.52 \\
\hline Simulated Sample Crosscheck Data 5000X Dilution & SRAT Receipt & 1.5 & Nitrate & $9 / 15$ & 1.39 & 1.46 \\
\hline Simulated Sample Crosscheck Data 5000X Dilution & SRAT Receipt & 1 & Sulfate & $9 / 15$ & 0.99 & 1.03 \\
\hline Simulated Sample Crosscheck Data 5000X Dilution & SRAT Receipt & 1 & Oxalate & $9 / 15$ & 1.06 & 1.09 \\
\hline Simulated Sample Crosscheck Data 5000X Dilution & SRAT Receipt & 1 & Phosphate & $9 / 15$ & 0.99 & 1.08 \\
\hline Simulated Sample Crosscheck Data 5000X Dilution & SRAT Receipt & 1 & Fluoride & $9 / 15$ & 1.02 & 1.02 \\
\hline Simulated Sample Crosscheck Data 5000X Dilution & SRAT Receipt & 1.5 & Formate & $9 / 15$ & 1.51 & 1.52 \\
\hline Simulated Sample Crosscheck Data 5000X Dilution & SRAT Receipt & 1 & Chloride & $9 / 15$ & 0.99 & 1 \\
\hline Simulated Sample Crosscheck Data 5000X Dilution & SRAT Receipt & 1.5 & Nitrite & $9 / 15$ & 1.49 & 1.52 \\
\hline $\begin{array}{l}\text { Simulated Sample Crosscheck Data 5000X Dilution } \\
\end{array}$ & SRAT Receipt & 1.5 & Nitrate & $9 / 15$ & 1.38 & 1.46 \\
\hline Simulated Sample Crosscheck Data 5000X Dilution & SRAT Receipt & 1 & Sulfate & $9 / 15$ & 1 & 1.03 \\
\hline Simulated Sample Crosscheck Data 5000X Dilution & SRAT Receipt & 1 & Oxalate & $9 / 15$ & 1.06 & 1.09 \\
\hline Simulated Sample Crosscheck Data 5000X Dilution & SRAT Receipt & 1 & Phosphate & $9 / 15$ & 1 & 1.08 \\
\hline Simulated Sample Crosscheck Data 5000X Dilution & SRAT Receipt & 1 & Fluoride & $9 / 15$ & 1.02 & 1.02 \\
\hline Simulated Sample Crosscheck Data 5000X Dilution & SRAT Receipt & 1.5 & Formate & $9 / 15$ & 1.51 & 1.52 \\
\hline $\begin{array}{l}\text { Simulated Sample Crosscheck Data 5000X Dilution } \\
\end{array}$ & SRAT Receipt & 1 & Chloride & $9 / 15$ & 0.96 & 1 \\
\hline Simulated Sample Crosscheck Data 5000X Dilution & SRAT Receipt & 1.5 & Nitrite & $9 / 15$ & 1.48 & 1.52 \\
\hline Simulated Sample Crosscheck Data 5000X Dilution & SRAT Receipt & 1.5 & Nitrate & $9 / 15$ & 1.37 & 1.47 \\
\hline Simulated Sample Crosscheck Data 5000X Dilution & SRAT Receipt & 1 & Sulfate & $9 / 15$ & 0.99 & 1.03 \\
\hline Simulated Sample Crosscheck Data 5000X Dilution & SRAT Receipt & 1 & Oxalate & $9 / 15$ & 1.06 & 1.1 \\
\hline Simulated Sample Crosscheck Data 5000X Dilution & SRAT Receipt & 1 & Phosphate & $9 / 15$ & 0.99 & 1.08 \\
\hline Simulated Sample Crosscheck Data 5000X Dilution & SRAT Receipt & 1 & Fluoride & $9 / 16$ & 1.02 & 1.02 \\
\hline Simulated Sample Crosscheck Data 5000X Dilution & SRAT Receipt & 1.5 & Formate & $9 / 16$ & 1.51 & 1.49 \\
\hline Simulated Sample Crosscheck Data 5000X Dilution & SRAT Receipt & 1 & Chloride & $9 / 16$ & 0.95 & 1 \\
\hline Simulated Sample Crosscheck Data 5000X Dilution & SRAT Receipt & 1.5 & Nitrite & $9 / 16$ & 1.48 & 1.51 \\
\hline Simulated Sample Crosscheck Data 5000X Dilution & SRAT Receipt & 1.5 & Nitrate & $9 / 16$ & 1.38 & 1.44 \\
\hline Simulated Sample Crosscheck Data 5000X Dilution & SRAT Receipt & 1 & Sulfate & $9 / 16$ & 0.99 & 1 \\
\hline
\end{tabular}


Table A2. Measurements of Simulants of SRAT Receipt, SRAT Product, and SME, of Standards Used for Calibration, and of Standards from Developed from Dried Salts

\begin{tabular}{|c|c|c|c|c|c|c|}
\hline Type & Simulant & \begin{tabular}{|c|} 
Known \\
Concentration (ppm)
\end{tabular} & Anion & Date & $\begin{array}{c}\text { M-14 ICS 3000 } \\
\text { Measurement (ppm) }\end{array}$ & $\begin{array}{c}\text { M-13 ICS 3000 } \\
\text { Measurement (ppm) }\end{array}$ \\
\hline Simulated Sample Crosscheck Data 5000X Dilution & SRAT Receipt & 1 & Oxalate & $9 / 16$ & 1.06 & 1.05 \\
\hline Simulated Sample Crosscheck Data 5000X Dilution & SRAT Receipt & 1 & Phosphate & $9 / 16$ & 0.98 & 1.06 \\
\hline Simulated Sample Crosscheck Data 5000X Dilution & SRAT Receipt & 1 & Fluoride & $9 / 16$ & 1.02 & 1.02 \\
\hline Simulated Sample Crosscheck Data 5000X Dilution & SRAT Receipt & 1.5 & Formate & $9 / 16$ & 1.51 & 1.5 \\
\hline Simulated Sample Crosscheck Data 5000X Dilution & SRAT Receipt & 1 & Chloride & $9 / 16$ & 0.95 & 1 \\
\hline Simulated Sample Crosscheck Data 5000X Dilution & SRAT Receipt & 1.5 & Nitrite & $9 / 16$ & 1.48 & 1.51 \\
\hline Simulated Sample Crosscheck Data 5000X Dilution & SRAT Receipt & 1.5 & Nitrate & $9 / 16$ & 1.38 & 1.44 \\
\hline Simulated Sample Crosscheck Data 5000X Dilution & SRAT Receipt & 1 & Sulfate & $9 / 16$ & 0.99 & 1 \\
\hline Simulated Sample Crosscheck Data 5000X Dilution & SRAT Receipt & 1 & Oxalate & $9 / 16$ & 1.06 & 1.06 \\
\hline Simulated Sample Crosscheck Data 5000X Dilution & SRAT Receipt & 1 & Phosphate & $9 / 16$ & 0.98 & 1.07 \\
\hline $1 \mathrm{ppm}$ Calibration Standard as a sample & standard & 1 & Fluoride & $9 / 15$ & 1.04 & 1.03 \\
\hline 1 ppm Calibration Standard as a sample & standard & 1 & Formate & $9 / 15$ & 1.03 & 0.96 \\
\hline 1 ppm Calibration Standard as a sample & standard & 1 & Chloride & $9 / 15$ & 0.97 & 1 \\
\hline 1 ppm Calibration Standard as a sample & standard & 1 & Nitrite & $9 / 15$ & 1 & 1.01 \\
\hline 1 ppm Calibration Standard as a sample & standard & 1 & Nitrate & $9 / 15$ & 1.03 & 1.07 \\
\hline $1 \mathrm{ppm}$ Calibration Standard as a sample & standard & 1 & Sulfate & $9 / 15$ & 1.01 & 1.03 \\
\hline 1 ppm Calibration Standard as a sample & standard & 1 & Oxalate & $9 / 15$ & 1.07 & 1.08 \\
\hline 1 ppm Calibration Standard as a sample & standard & 1 & Phosphate & $9 / 15$ & 1.01 & 1.06 \\
\hline 1 ppm Calibration Standard as a sample & standard & 1 & Fluoride & $9 / 16$ & 1.03 & 1.02 \\
\hline $1 \mathrm{ppm}$ Calibration Standard as a sample & standard & 1 & Formate & $9 / 16$ & 1.01 & 1 \\
\hline $1 \mathrm{ppm}$ Calibration Standard as a sample & standard & 1 & Chloride & $9 / 16$ & 0.95 & 0.99 \\
\hline $1 \mathrm{ppm}$ Calibration Standard as a sample & standard & 1 & Nitrite & $9 / 16$ & 0.97 & 1 \\
\hline $1 \mathrm{ppm}$ Calibration Standard as a sample & standard & 1 & Nitrate & $9 / 16$ & 1 & 1.06 \\
\hline 1 ppm Calibration Standard as a sample & standard & 1 & Sulfate & $9 / 16$ & 0.99 & 1.01 \\
\hline $1 \mathrm{ppm}$ Calibration Standard as a sample & standard & 1 & Oxalate & $9 / 16$ & 1.05 & 1.07 \\
\hline 1 ppm Calibration Standard as a sample & standard & 1 & Phosphate & $9 / 16$ & 0.97 & 1.09 \\
\hline $1 \mathrm{ppm}$ Calibration Standard as a sample & standard & 1 & $\begin{array}{l}\text { Fluoride } \\
\end{array}$ & $9 / 16$ & 1.03 & 1.02 \\
\hline $1 \mathrm{ppm}$ Calibration Standard as a sample & standard & 1 & Formate & $9 / 16$ & 1.01 & 1 \\
\hline $1 \mathrm{ppm}$ Calibration Standard as a sample & standard & 1 & Chloride & $9 / 16$ & 0.94 & 0.99 \\
\hline $1 \mathrm{ppm}$ Calibration Standard as a sample & standard & 1 & Nitrite & $9 / 16$ & 0.96 & 1.01 \\
\hline $1 \mathrm{ppm}$ Calibration Standard as a sample & standard & 1 & Nitrate & $9 / 16$ & 0.99 & 1.06 \\
\hline $1 \mathrm{ppm}$ Calibration Standard as a sample & standard & 1 & Sulfate & $9 / 16$ & 0.99 & 1.01 \\
\hline $1 \mathrm{ppm}$ Calibration Standard as a sample & standard & 1 & Oxalate & $9 / 16$ & 1.05 & 1.07 \\
\hline $1 \mathrm{ppm}$ Calibration Standard as a sample & standard & 1 & Phosphate & $9 / 16$ & 0.97 & 1.1 \\
\hline $10 \mathrm{ppm}$ Calibration Standard as a sample & standard & 10 & Fluoride & $9 / 15$ & 9.97 & 10 \\
\hline $10 \mathrm{ppm}$ Calibration Standard as a sample & standard & 10 & Formate & $9 / 15$ & 9.96 & 10.47 \\
\hline $10 \mathrm{ppm}$ Calibration Standard as a sample & standard & 10 & Chloride & $9 / 15$ & 9.99 & 10.01 \\
\hline $10 \mathrm{ppm}$ Calibration Standard as a sample & standard & 10 & Nitrite & $9 / 15$ & 10.01 & 10.02 \\
\hline $10 \mathrm{ppm}$ Calibration Standard as a sample & standard & 10 & Nitrate & $9 / 15$ & 10.03 & 10.07 \\
\hline $10 \mathrm{ppm}$ Calibration Standard as a sample & standard & 10 & Sulfate & $9 / 15$ & 10.02 & 10.11 \\
\hline $10 \mathrm{ppm}$ Calibration Standard as a sample & standard & 10 & Oxalate & $9 / 15$ & 10.12 & 10.09 \\
\hline $10 \mathrm{ppm}$ Calibration Standard as a sample & standard & 10 & Phosphate & $9 / 15$ & 10.03 & 10.34 \\
\hline $10 \mathrm{ppm}$ Calibration Standard as a sample & standard & 10 & Fluoride & $9 / 16$ & 9.98 & 9.97 \\
\hline $10 \mathrm{ppm}$ Calibration Standard as a sample & standard & 10 & Formate & $9 / 16$ & 9.97 & 10.03 \\
\hline $10 \mathrm{ppm}$ Calibration Standard as a sample & standard & 10 & Chloride & $9 / 16$ & 9.95 & 9.99 \\
\hline $10 \mathrm{ppm}$ Calibration Standard as a sample & standard & 10 & Nitrite & $9 / 16$ & 9.93 & 10.02 \\
\hline $10 \mathrm{ppm}$ Calibration Standard as a sample & standard & 10 & Nitrate & $9 / 16$ & 9.94 & 10.03 \\
\hline $10 \mathrm{ppm}$ Calibration Standard as a sample & standard & 10 & Sulfate & $9 / 16$ & 9.97 & 10.07 \\
\hline $10 \mathrm{ppm}$ Calibration Standard as a sample & standard & 10 & Oxalate & $9 / 16$ & 10.17 & 10.14 \\
\hline $10 \mathrm{ppm}$ Calibration Standard as a sample & standard & 10 & Phosphate & $9 / 16$ & 10.02 & 10.24 \\
\hline
\end{tabular}


Table A2. Measurements of Simulants of SRAT Receipt, SRAT Product, and SME, of Standards Used for Calibration, and of Standards from Developed from Dried Salts

\begin{tabular}{|c|c|c|c|c|c|c|}
\hline Type & Simulant & \begin{tabular}{|c|} 
Known \\
Concentration (ppm)
\end{tabular} & Anion & Date & $\begin{array}{c}\text { M-14 ICS 3000 } \\
\text { Measurement (ppm) }\end{array}$ & $\begin{array}{c}\text { M-13 ICS 3000 } \\
\text { Measurement (ppm) }\end{array}$ \\
\hline $10 \mathrm{ppm}$ Calibration Standard as a sample & standard & 10 & Fluoride & $9 / 16$ & 9.97 & 9.98 \\
\hline $10 \mathrm{ppm}$ Calibration Standard as a sample & standard & 10 & Formate & $9 / 16$ & 9.97 & 10.04 \\
\hline $10 \mathrm{ppm}$ Calibration Standard as a sample & standard & 10 & Chloride & $9 / 16$ & 9.96 & 9.99 \\
\hline $10 \mathrm{ppm}$ Calibration Standard as a sample & standard & 10 & Nitrite & $9 / 16$ & 9.94 & 10.04 \\
\hline $10 \mathrm{ppm}$ Calibration Standard as a sample & standard & 10 & Nitrate & $9 / 16$ & 9.95 & 10.04 \\
\hline $10 \mathrm{ppm}$ Calibration Standard as a sample & standard & 10 & Sulfate & $9 / 16$ & 9.97 & 10.08 \\
\hline $10 \mathrm{ppm}$ Calibration Standard as a sample & standard & 10 & Oxalate & $9 / 16$ & 10.18 & 10.14 \\
\hline $10 \mathrm{ppm}$ Calibration Standard as a sample & standard & 10 & Phosphate & $9 / 16$ & 10.03 & 10.26 \\
\hline $20 \mathrm{ppm}$ Calibration Standard as a sample & standard & 20 & Fluoride & $9 / 15$ & 19.9 & 20.04 \\
\hline $20 \mathrm{ppm}$ Calibration Standard as a sample & standard & 20 & Formate & $9 / 15$ & 19.89 & 19.81 \\
\hline $20 \mathrm{ppm}$ Calibration Standard as a sample & standard & 20 & Chloride & $9 / 15$ & 19.96 & 20.01 \\
\hline $20 \mathrm{ppm}$ Calibration Standard as a sample & standard & 20 & Nitrite & $9 / 15$ & 19.99 & 19.97 \\
\hline $20 \mathrm{ppm}$ Calibration Standard as a sample & standard & 20 & Nitrate & $9 / 15$ & 19.99 & 20.06 \\
\hline $20 \mathrm{ppm}$ Calibration Standard as a sample & standard & 20 & Sulfate & $9 / 15$ & 19.98 & 20.1 \\
\hline $20 \mathrm{ppm}$ Calibration Standard as a sample & standard & 20 & Oxalate & $9 / 15$ & 20.27 & 20.06 \\
\hline $20 \mathrm{ppm}$ Calibration Standard as a sample & standard & 20 & Phosphate & $9 / 15$ & 20.03 & 20.32 \\
\hline $20 \mathrm{ppm}$ Calibration Standard as a sample & standard & 20 & Fluoride & $9 / 16$ & 19.95 & 19.98 \\
\hline $20 \mathrm{ppm}$ Calibration Standard as a sample & standard & 20 & Formate & $9 / 16$ & 19.92 & 20.14 \\
\hline $20 \mathrm{ppm}$ Calibration Standard as a sample & standard & 20 & Chloride & $9 / 16$ & 19.92 & 20 \\
\hline $20 \mathrm{ppm}$ Calibration Standard as a sample & standard & 20 & Nitrite & $9 / 16$ & 19.86 & 20.13 \\
\hline $20 \mathrm{ppm}$ Calibration Standard as a sample & standard & 20 & Nitrate & $9 / 16$ & 19.93 & 20.04 \\
\hline $20 \mathrm{ppm}$ Calibration Standard as a sample & standard & 20 & Sulfate & $9 / 16$ & 19.88 & 20.04 \\
\hline $20 \mathrm{ppm}$ Calibration Standard as a sample & standard & 20 & Oxalate & $9 / 16$ & 20.31 & 20.21 \\
\hline $20 \mathrm{ppm}$ Calibration Standard as a sample & standard & 20 & Phosphate & $9 / 16$ & 20.04 & 20.26 \\
\hline $20 \mathrm{ppm}$ Calibration Standard as a sample & standard & 20 & Fluoride & $9 / 16$ & 19.96 & 19.98 \\
\hline $20 \mathrm{ppm}$ Calibration Standard as a sample & standard & 20 & Formate & $9 / 16$ & 19.93 & 20.14 \\
\hline $20 \mathrm{ppm}$ Calibration Standard as a sample & standard & 20 & Chloride & $9 / 16$ & 19.93 & 19.99 \\
\hline $20 \mathrm{ppm}$ Calibration Standard as a sample & standard & 20 & Nitrite & $9 / 16$ & 19.85 & 20 \\
\hline $20 \mathrm{ppm}$ Calibration Standard as a sample & standard & 20 & Nitrate & $9 / 16$ & 19.95 & 20.02 \\
\hline $20 \mathrm{ppm}$ Calibration Standard as a sample & standard & 20 & Sulfate & $9 / 16$ & 19.91 & 20.04 \\
\hline $20 \mathrm{ppm}$ Calibration Standard as a sample & standard & 20 & Oxalate & $9 / 16$ & 20.34 & 20.22 \\
\hline $20 \mathrm{ppm}$ Calibration Standard as a sample & standard & 20 & Phosphate & $9 / 16$ & 20.06 & 20.26 \\
\hline $10 \mathrm{ppm}$ Standards prepared from dried salts & standard & 10 & Fluoride & $9 / 17$ & 10.12 & 9.96 \\
\hline $10 \mathrm{ppm}$ Standards prepared from dried salts & standard & 10 & Formate & $9 / 17$ & 10.3 & 9.89 \\
\hline $10 \mathrm{ppm}$ Standards prepared from dried salts & standard & 10 & Chloride & $9 / 17$ & 10 & 9.77 \\
\hline $10 \mathrm{ppm}$ Standards prepared from dried salts & standard & 10 & Nitrite & $9 / 17$ & 10.58 & 10.25 \\
\hline $10 \mathrm{ppm}$ Standards prepared from dried salts & standard & 10 & Nitrate & $9 / 17$ & 9.89 & 10.01 \\
\hline $10 \mathrm{ppm}$ Standards prepared from dried salts & standard & 10 & Sulfate & $9 / 17$ & 10.29 & 10.23 \\
\hline $10 \mathrm{ppm}$ Standards prepared from dried salts & standard & 10 & Oxalate & $9 / 17$ & 10.29 & 10.11 \\
\hline $10 \mathrm{ppm}$ Standards prepared from dried salts & standard & 10 & Phosphate & $9 / 17$ & 9.54 & 10.06 \\
\hline $10 \mathrm{ppm}$ Standards prepared from dried salts & standard & 10 & Fluoride & $9 / 17$ & 10.13 & 9.97 \\
\hline $10 \mathrm{ppm}$ Standards prepared from dried salts & standard & 10 & Formate & $9 / 17$ & 10.3 & 9.89 \\
\hline $10 \mathrm{ppm}$ Standards prepared from dried salts & standard & 10 & Chloride & $9 / 17$ & 9.97 & 9.75 \\
\hline $10 \mathrm{ppm}$ Standards prepared from dried salts & standard & 10 & Nitrite & $9 / 17$ & 10.58 & 10.25 \\
\hline $10 \mathrm{ppm}$ Standards prepared from dried salts & standard & 10 & Nitrate & $9 / 17$ & 9.89 & 9.96 \\
\hline $10 \mathrm{ppm}$ Standards prepared from dried salts & standard & 10 & Sulfate & $9 / 17$ & 10.18 & 10.14 \\
\hline $10 \mathrm{ppm}$ Standards prepared from dried salts & standard & 10 & Oxalate & $9 / 17$ & 10.3 & 10.05 \\
\hline $10 \mathrm{ppm}$ Standards prepared from dried salts & standard & 10 & Phosphate & $9 / 17$ & 9.55 & 9.63 \\
\hline
\end{tabular}


Exhibit A1.a Type of Sample=standard, Anion=Chloride, Bottle ID $=16 \mathrm{ppm}$

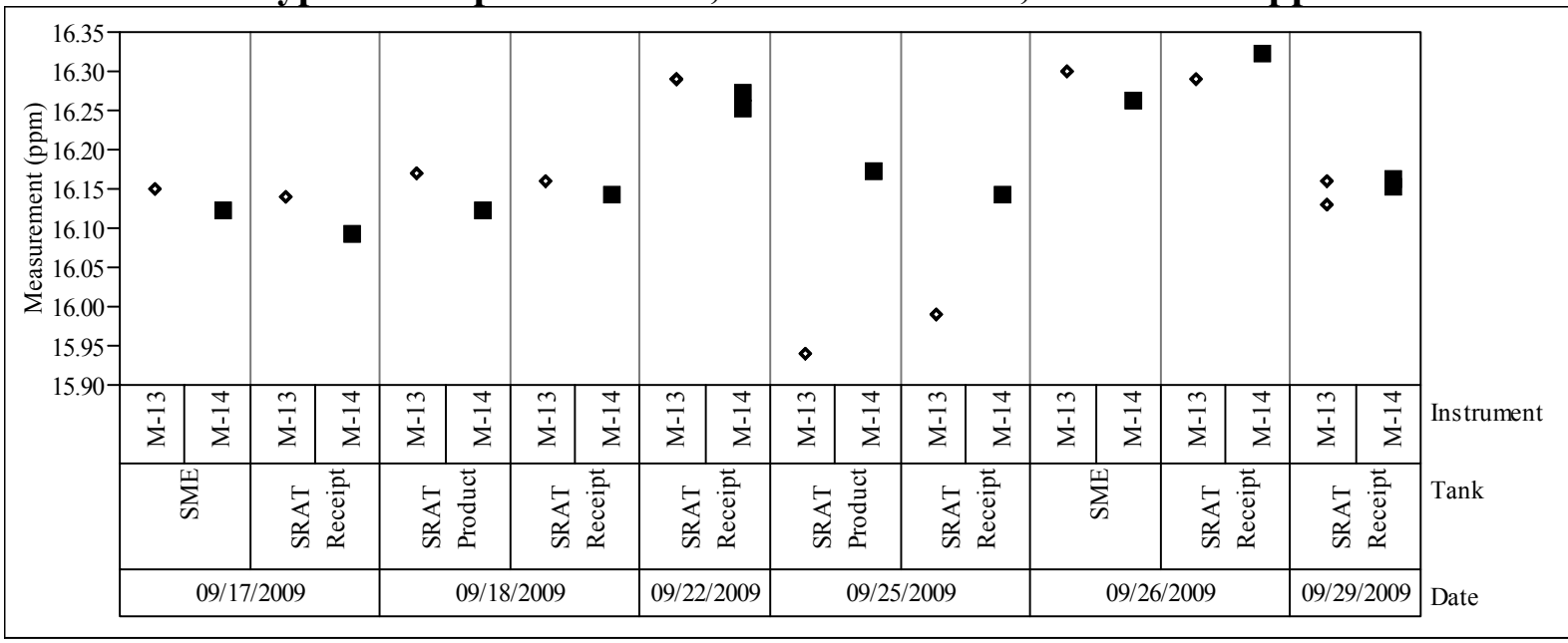

Exhibit A1b. Type of Sample=standard, Anion=Chloride, Bottle ID=2ppm

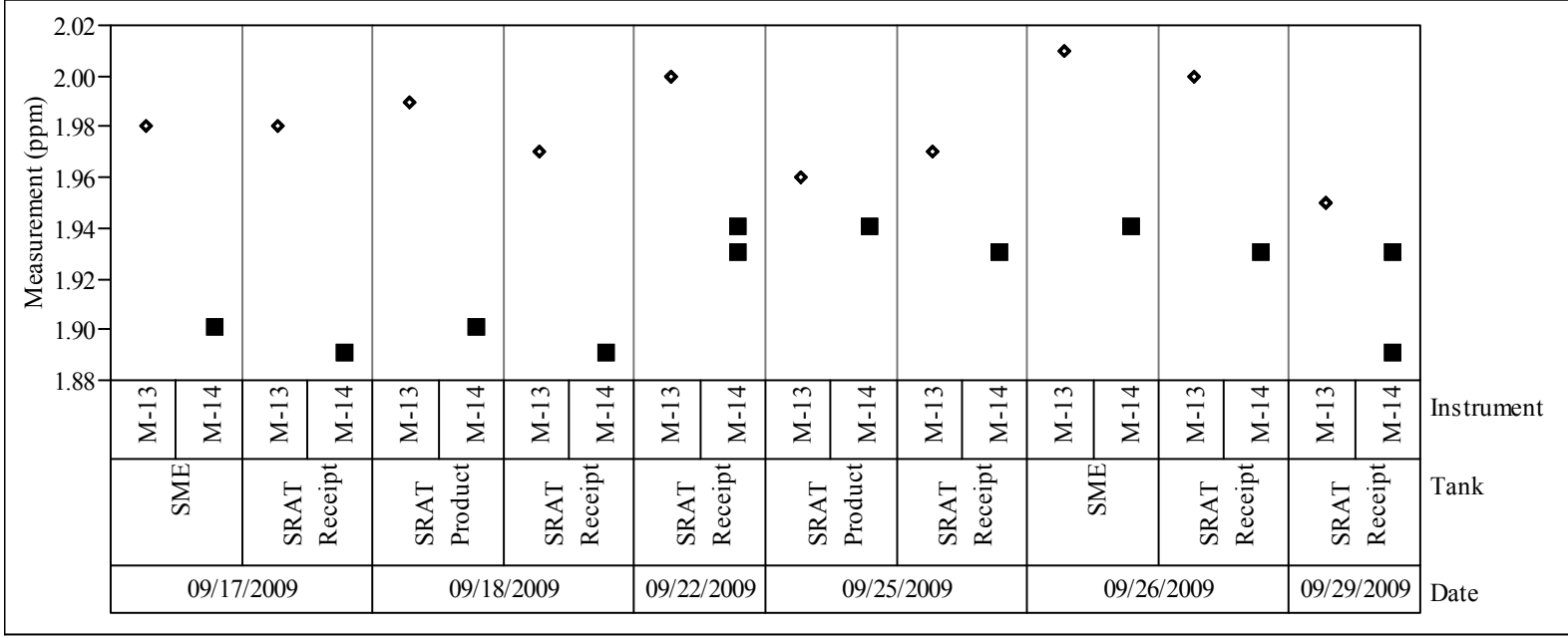

Exhibit A1c. Type of Sample=standard, Anion=Fluoride, Bottle ID=16ppm

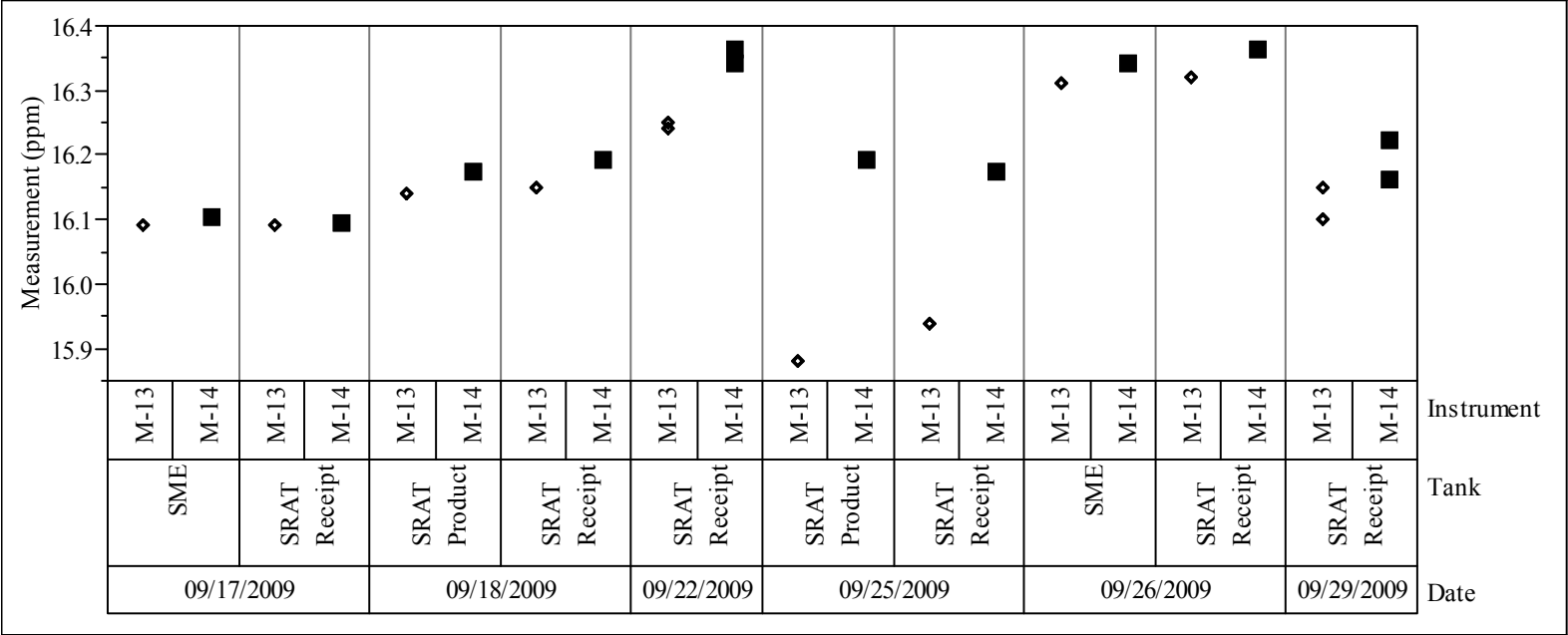


Exhibit A1d. Type of Sample=standard, Anion=Fluoride, Bottle ID=2ppm

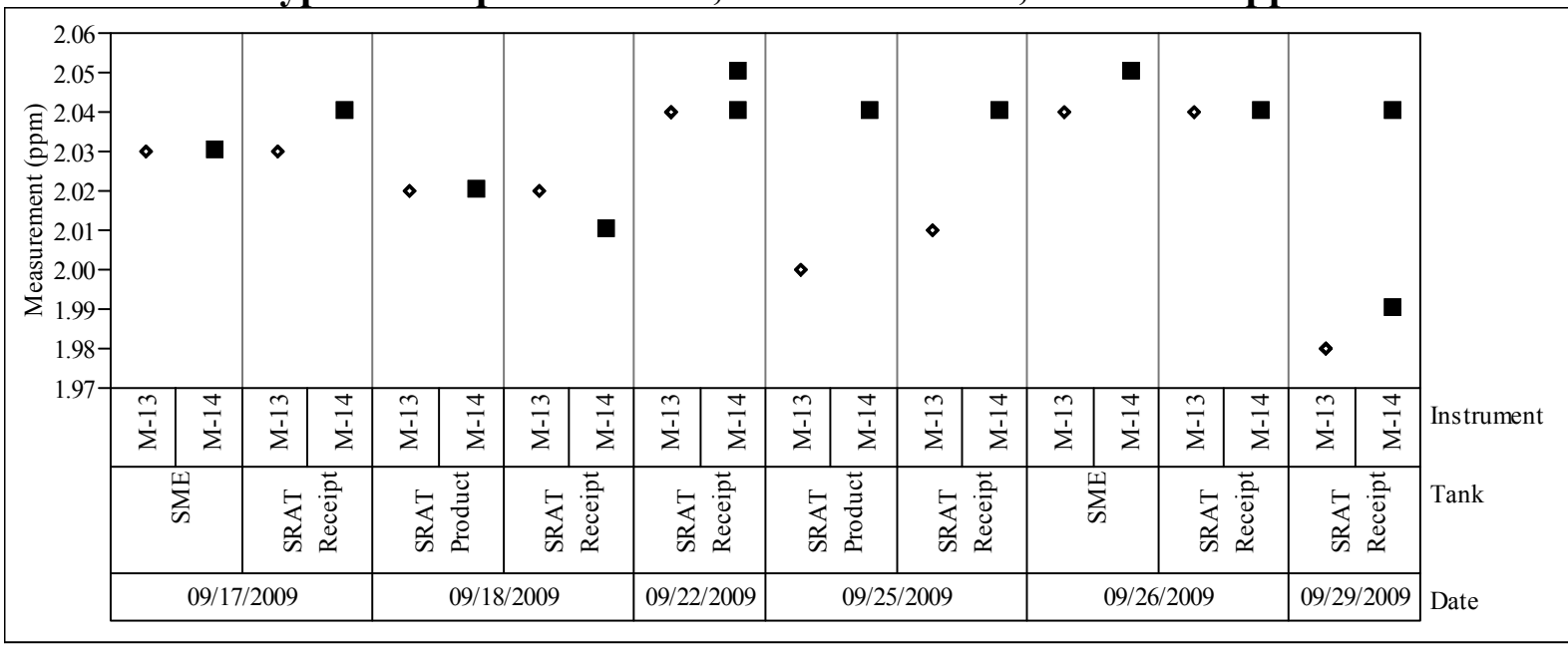

Exhibit A1e. Type of Sample=standard, Anion=Formate, Bottle ID=16ppm

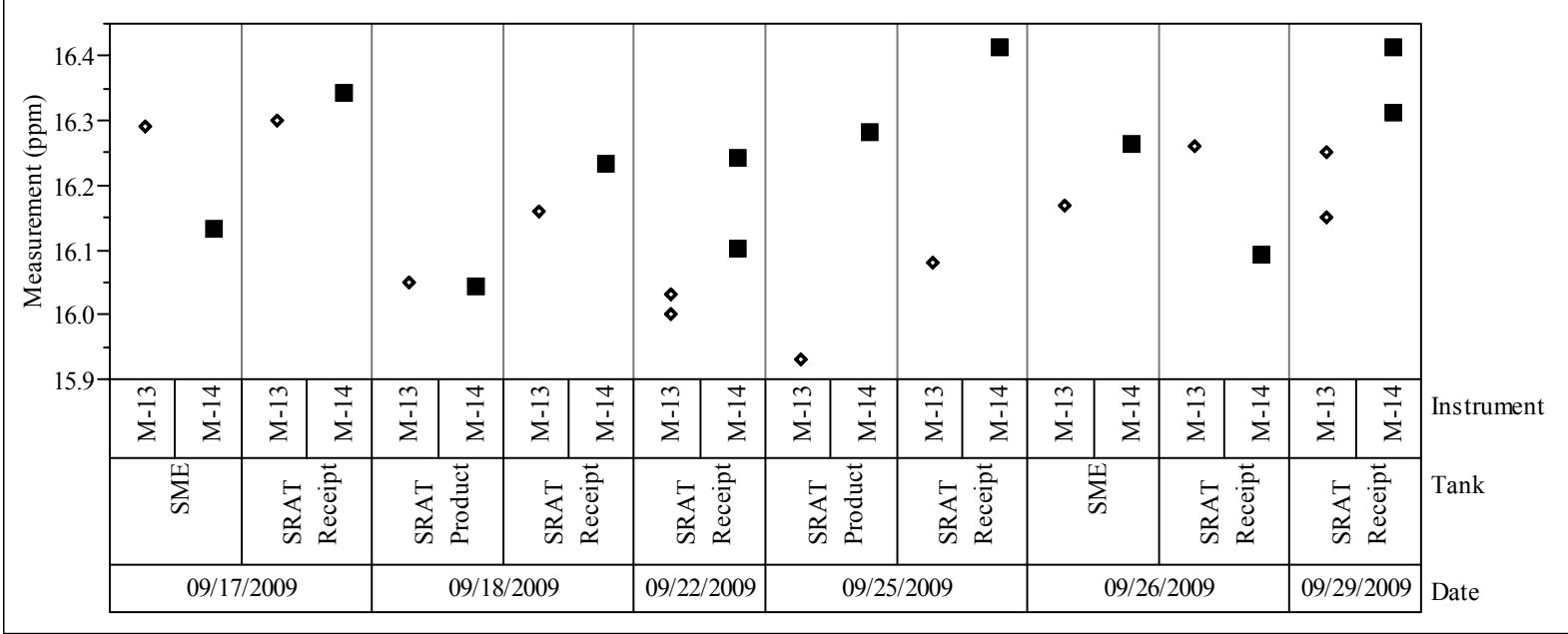

Exhibit A1f. Type of Sample=standard, Anion=Formate, Bottle ID=2ppm

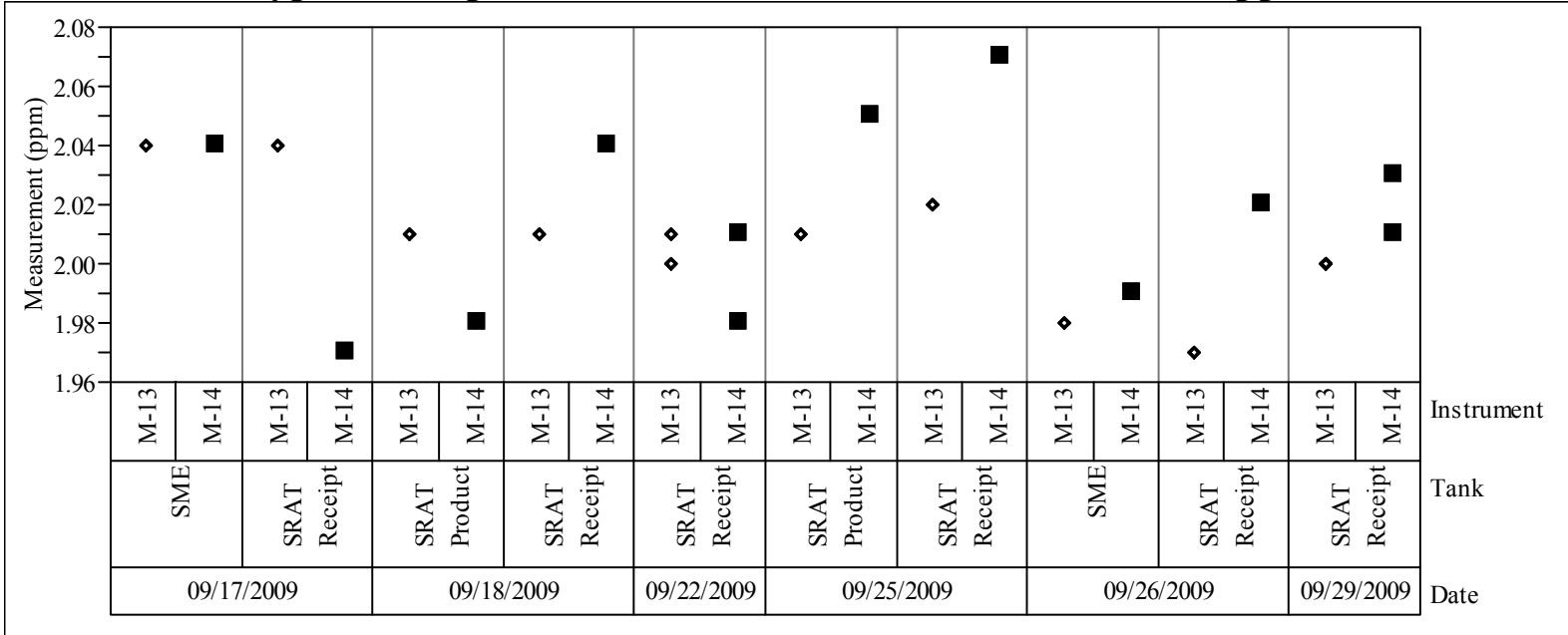


Exhibit A1g. Type of Sample=standard, Anion=Nitrate, Bottle ID=16ppm

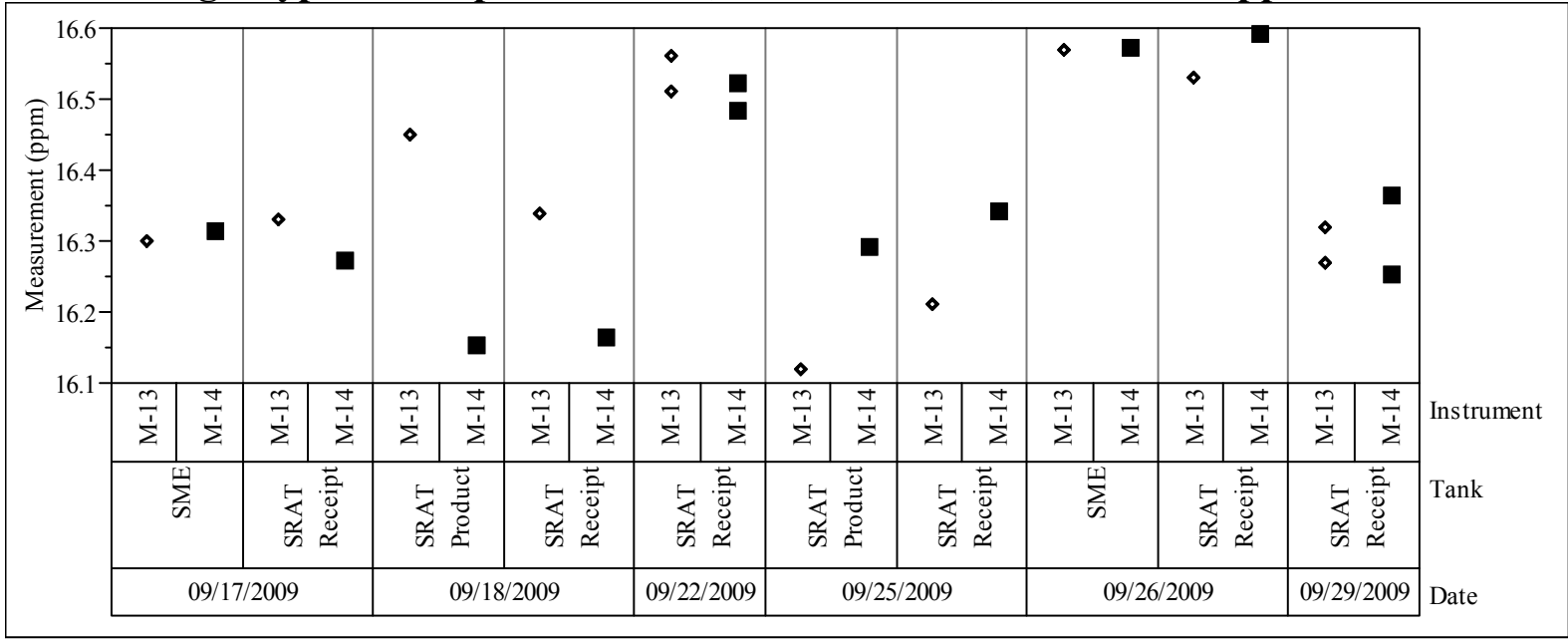

Exhibit A1h. Type of Sample=standard, Anion=Nitrate, Bottle ID=2ppm

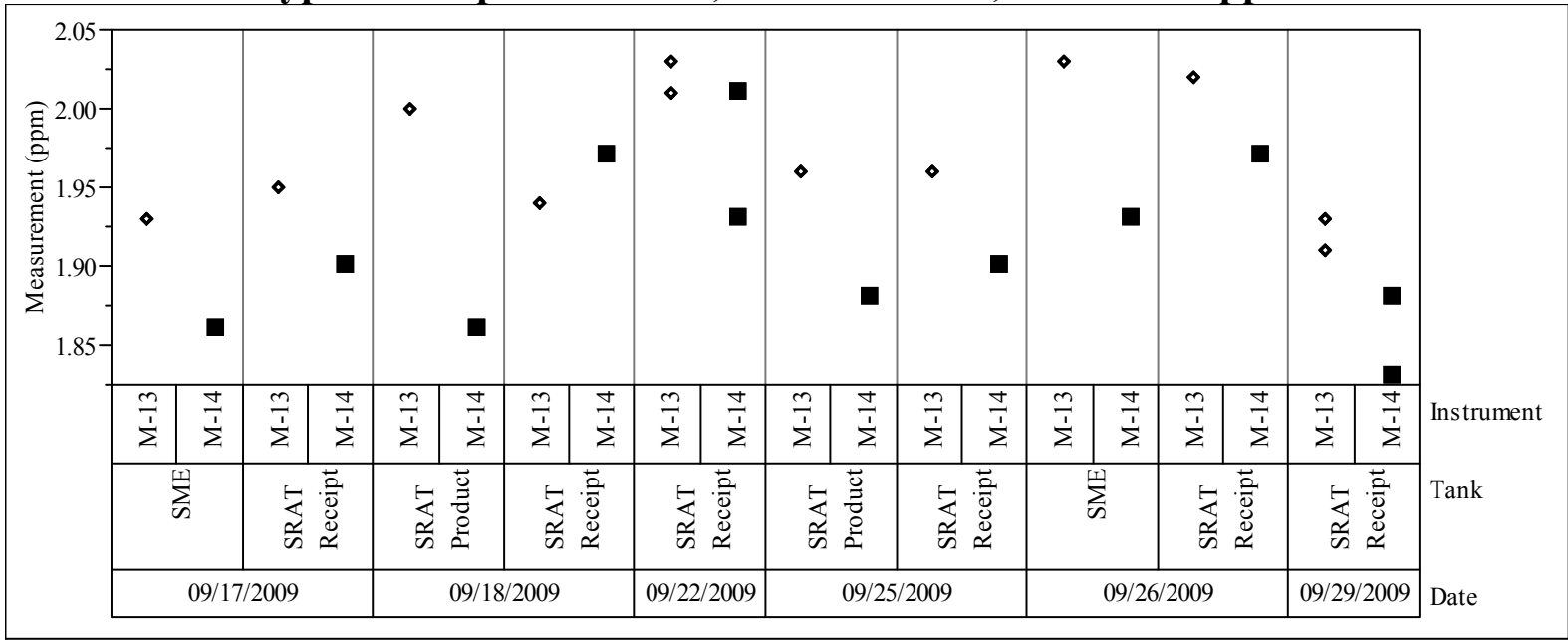

Exhibit A1i. Type of Sample=standard, Anion=Nitrite, Bottle ID=16ppm

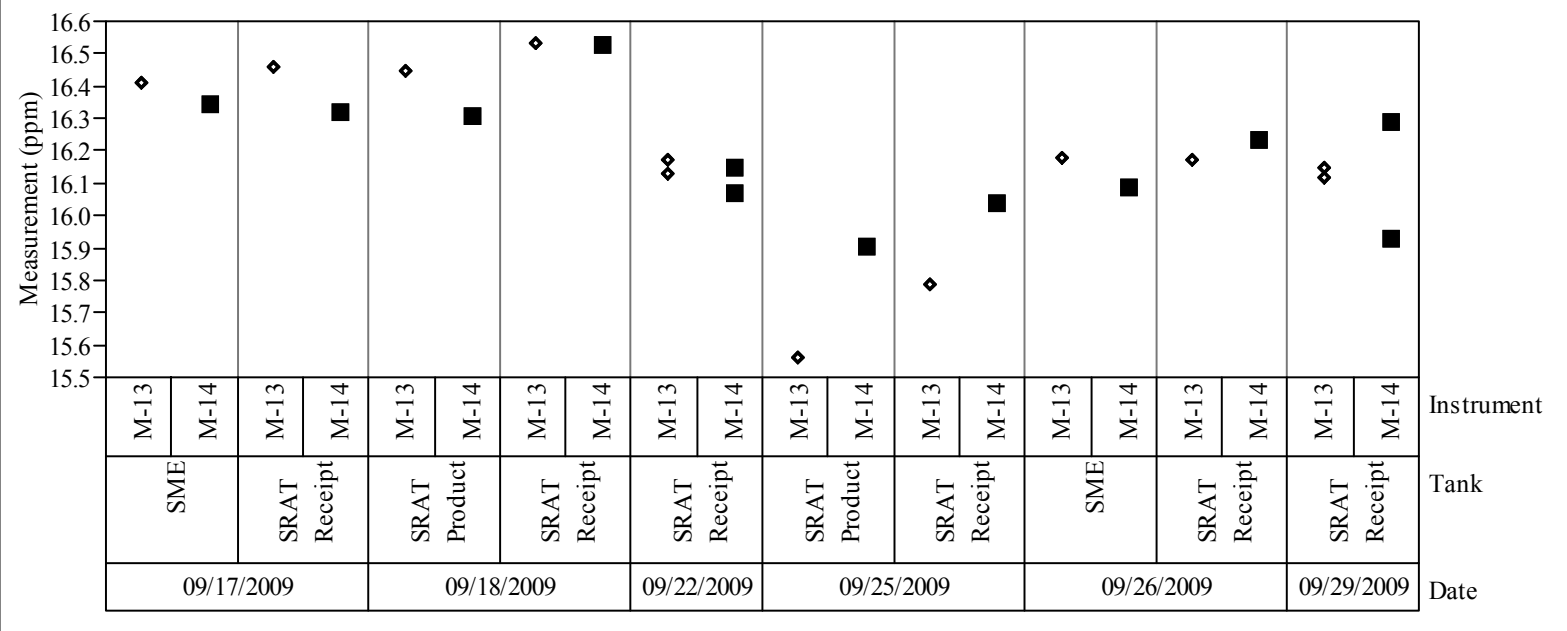


Exhibit A1j. Type of Sample=standard, Anion=Nitrite, Bottle ID=2ppm

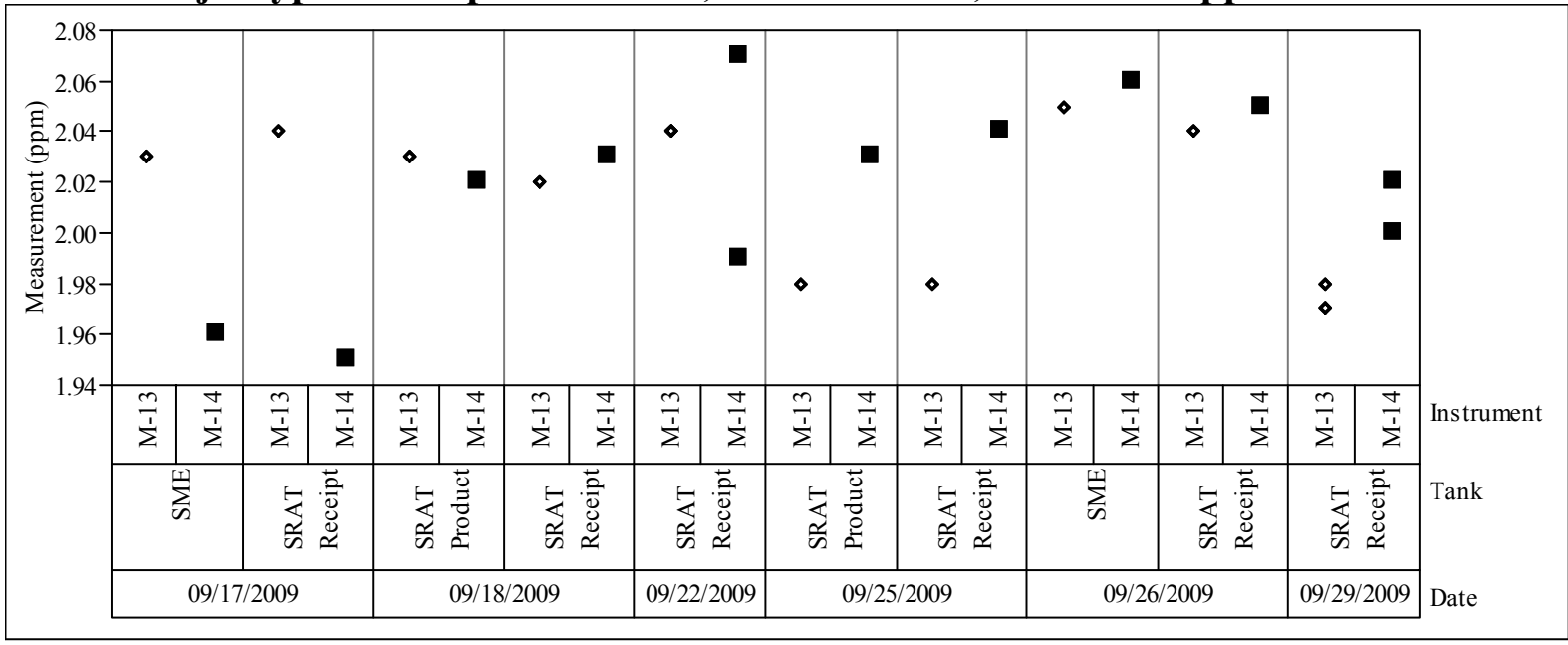

Exhibit A1k. Type of Sample=standard, Anion=Oxalate, Bottle ID=16ppm

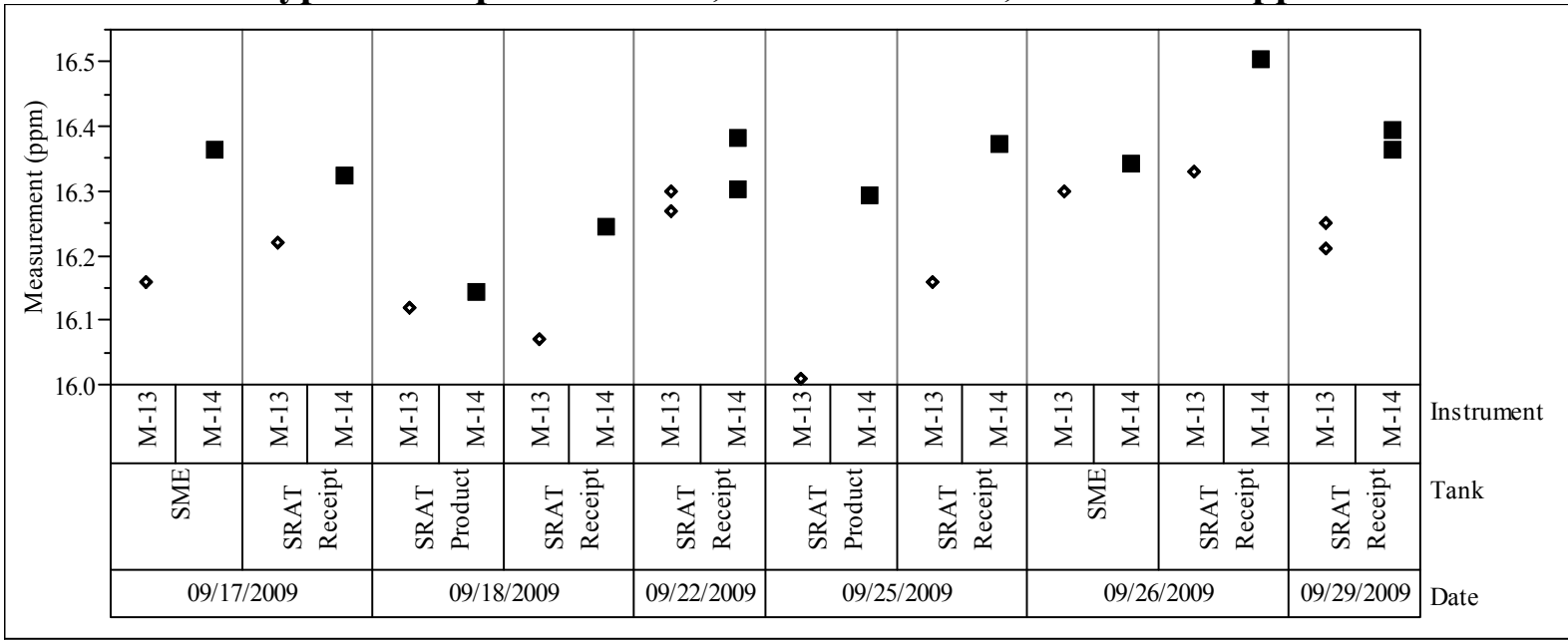

Exhibit A11. Type of Sample=standard, Anion=Oxalate, Bottle ID=2ppm

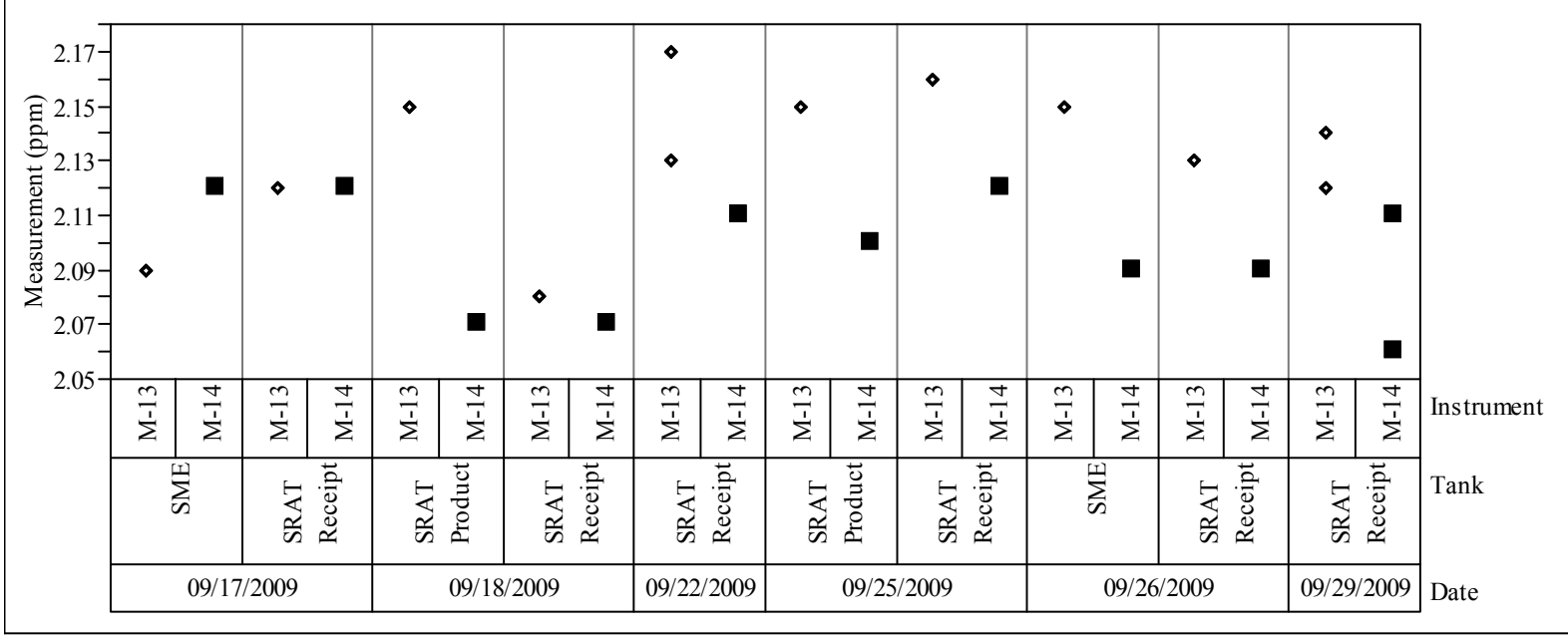


Exhibit A1m. Type of Sample=standard, Anion=Phosphate, Bottle ID=16ppm

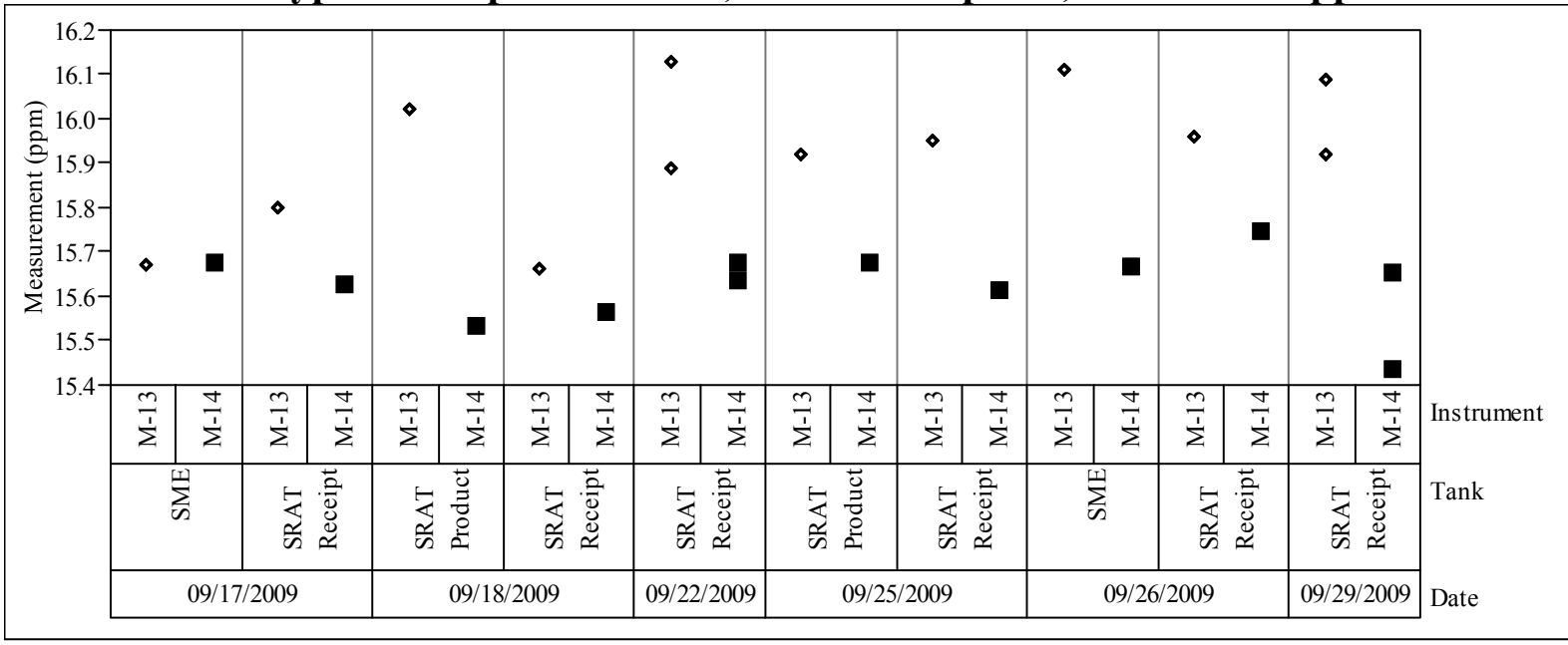

Exhibit A1n. Type of Sample=standard, Anion=Phosphate, Bottle ID=2ppm

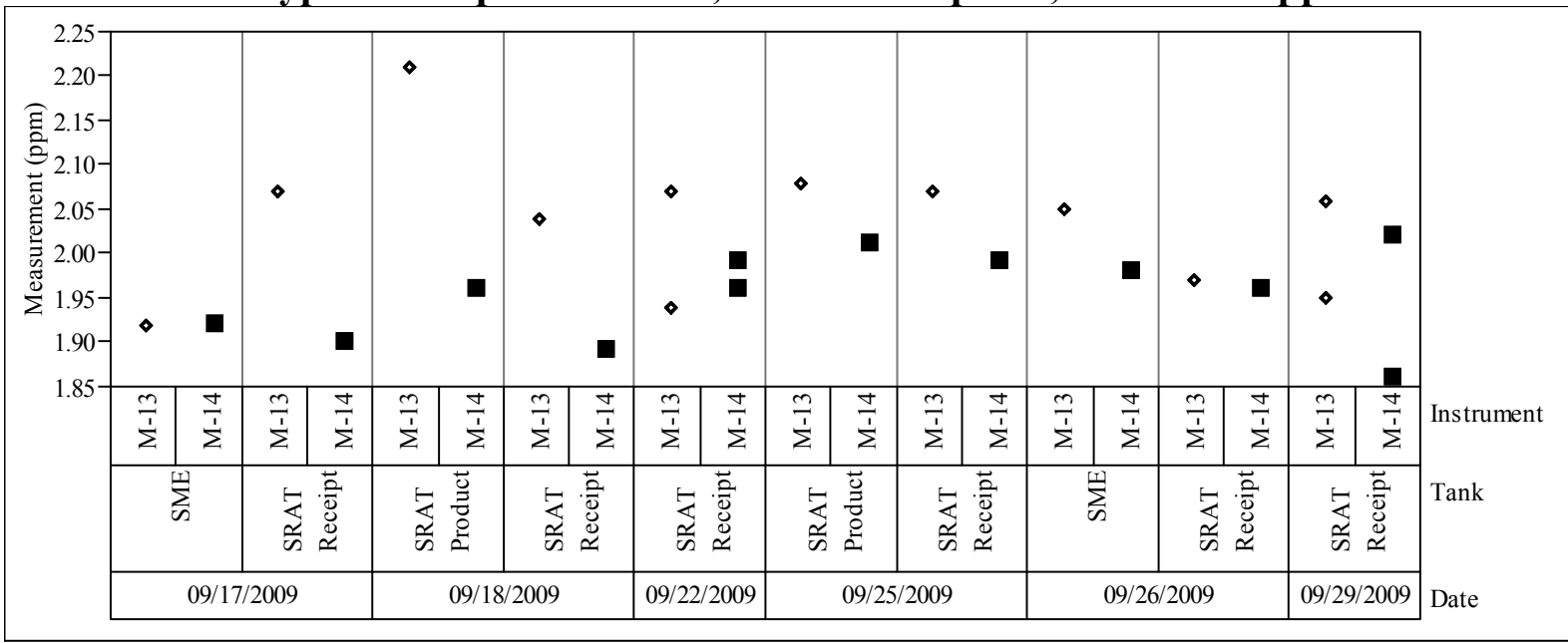

Exhibit A10. Type of Sample=standard, Anion=Sulfate, Bottle ID=16ppm

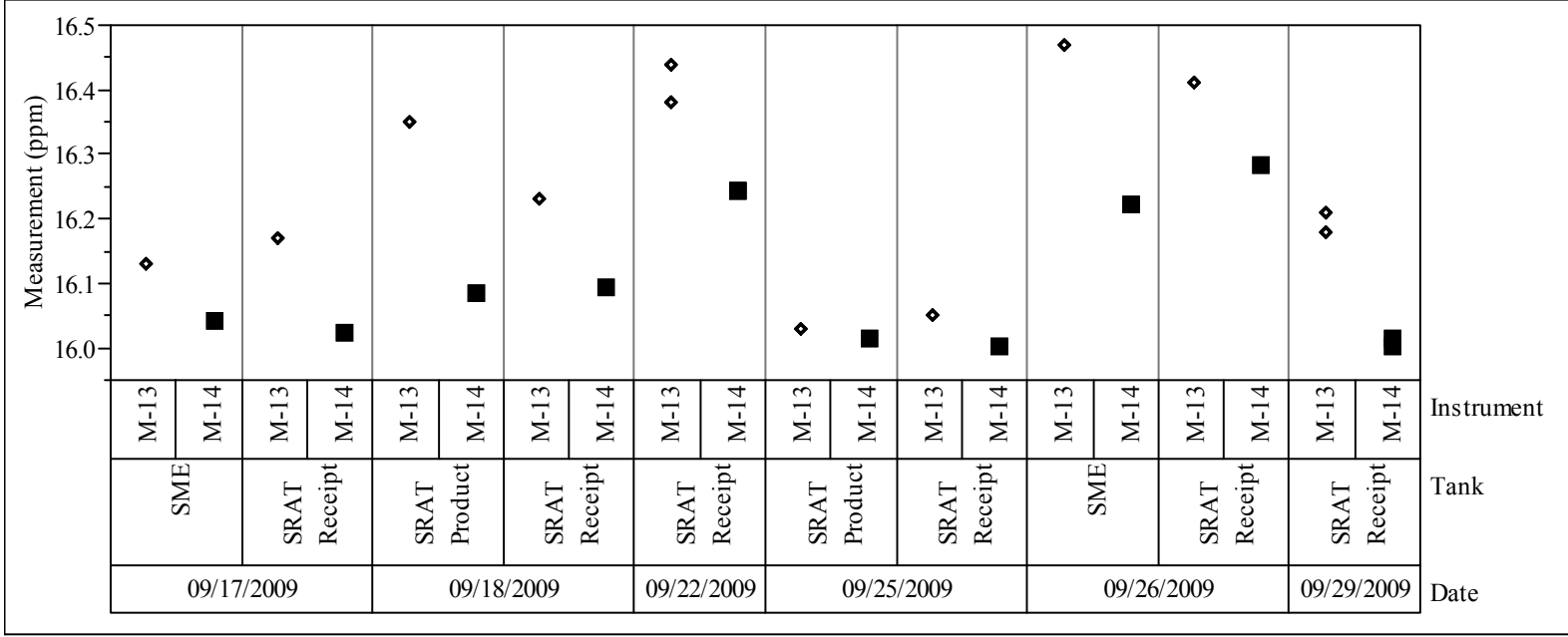


Exhibit A1p. Type of Sample=standard, Anion=Sulfate, Bottle ID=2ppm

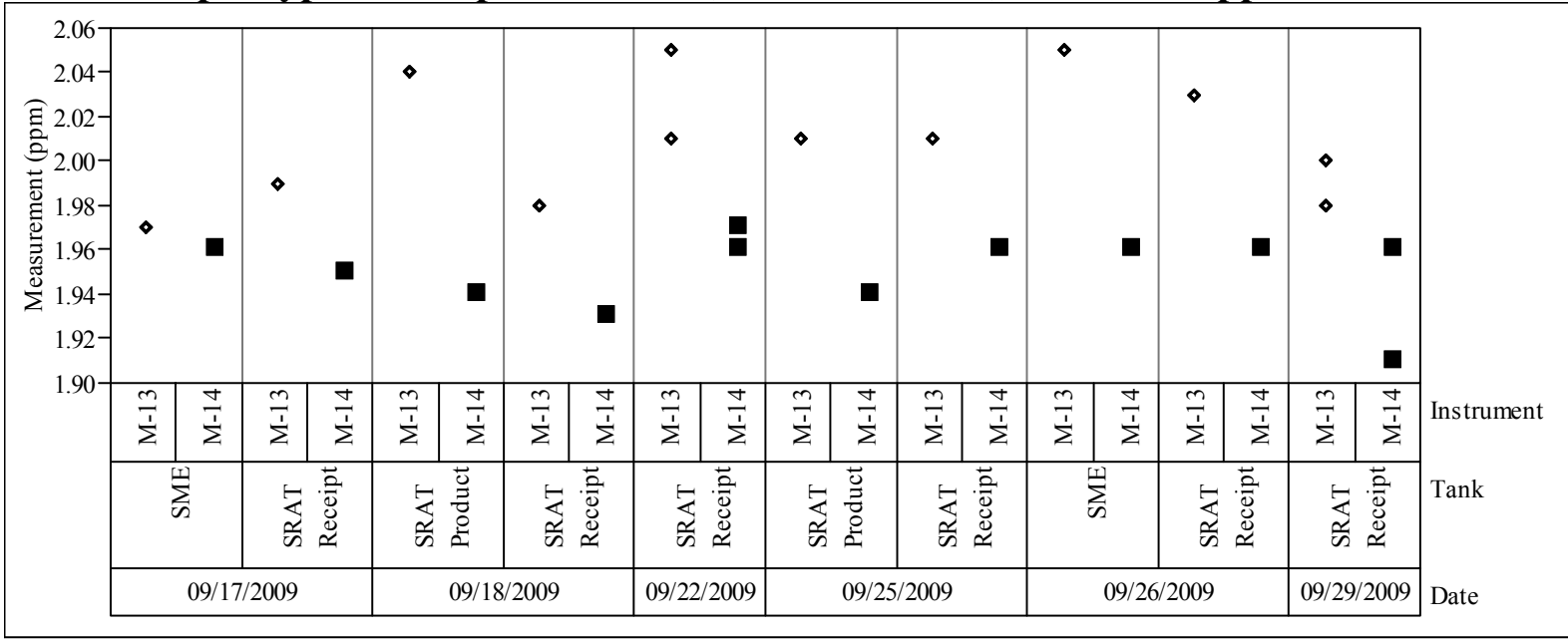

Exhibit A2a. Type of Sample=process, Anion=Formate; Raw Score Difference (ppm) (M13-M14)

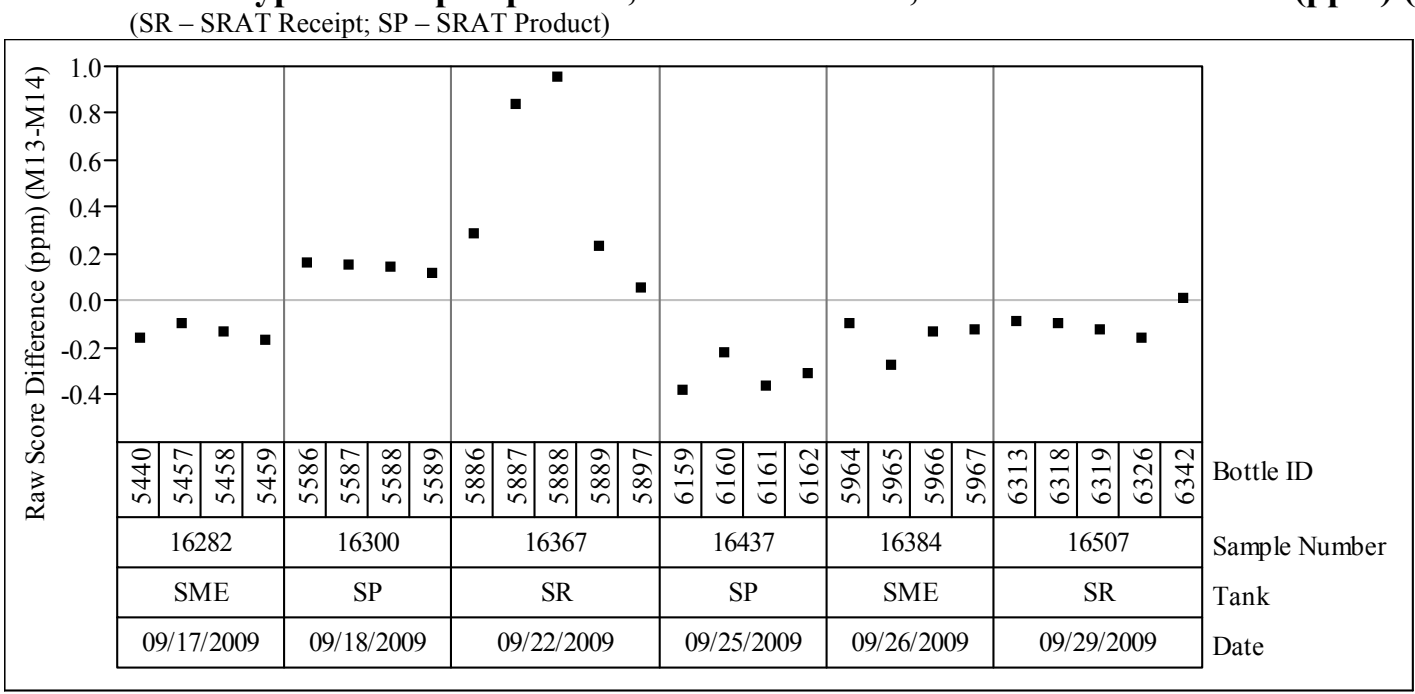

Exhibit A2b. Type of Sample=process, Anion=Nitrate; Raw Score Difference (ppm) (M13-M14) (SR - SRAT Receipt; SP - SRAT Product)

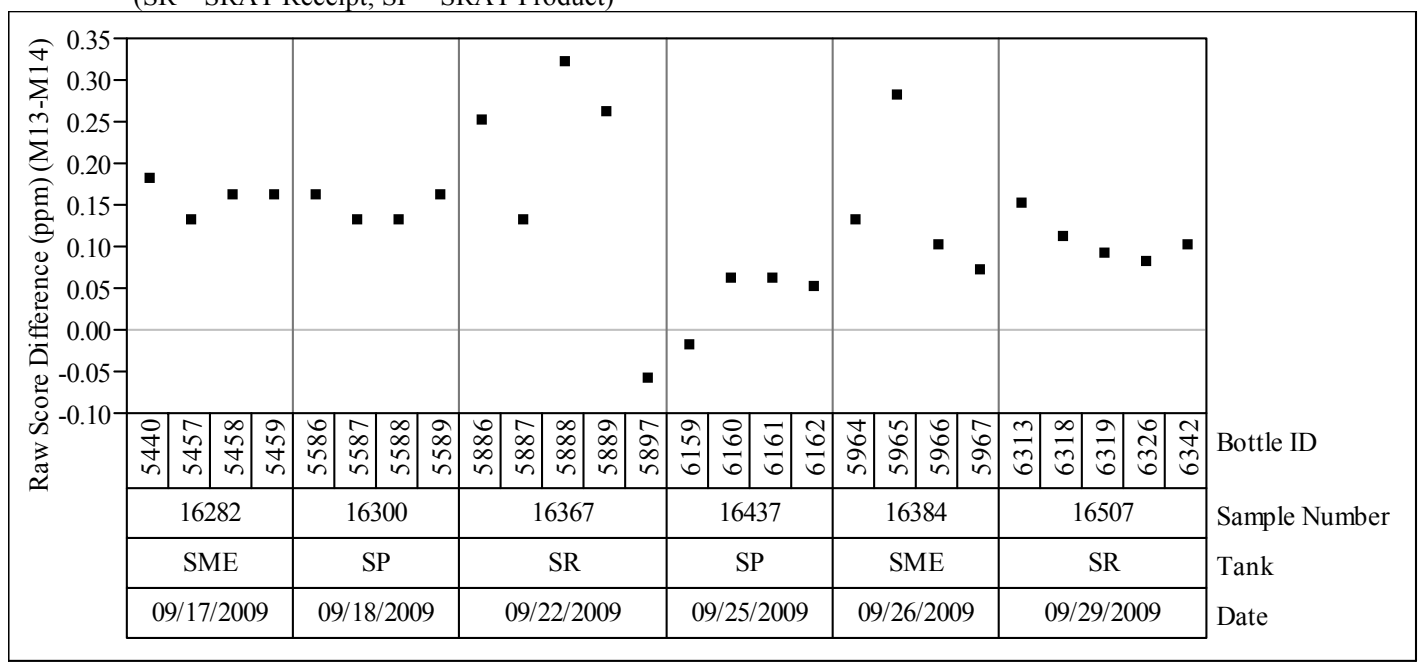


Exhibit A2c. Type of Sample=process, Anion=Nitrite; Raw Score Difference (ppm) (M13-M14) (SR - SRAT Receipt)

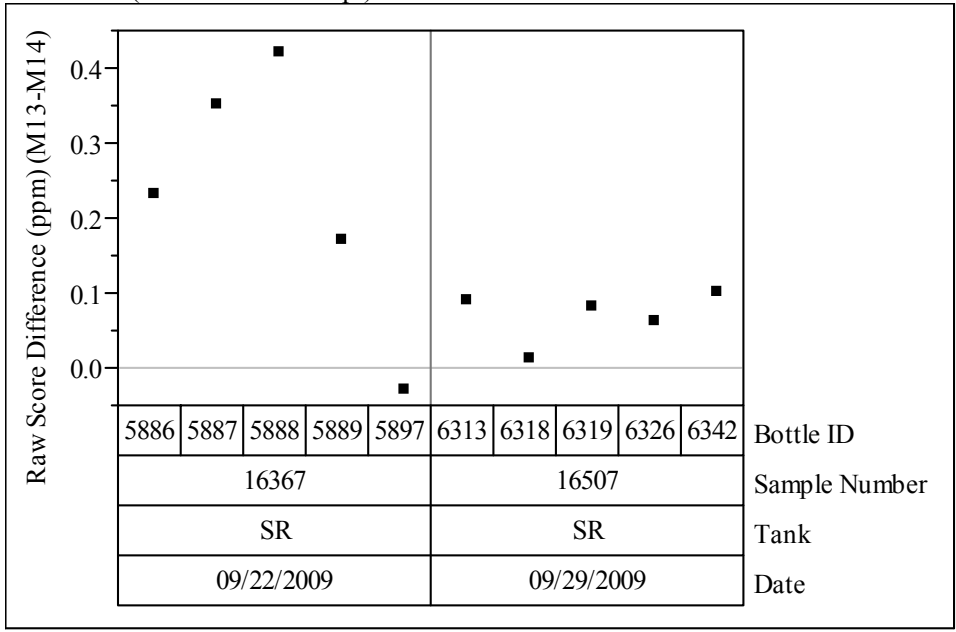

Exhibit A2d. Type of Sample=process, Anion=Oxalate; Raw Score Difference (ppm) (M13-M14)

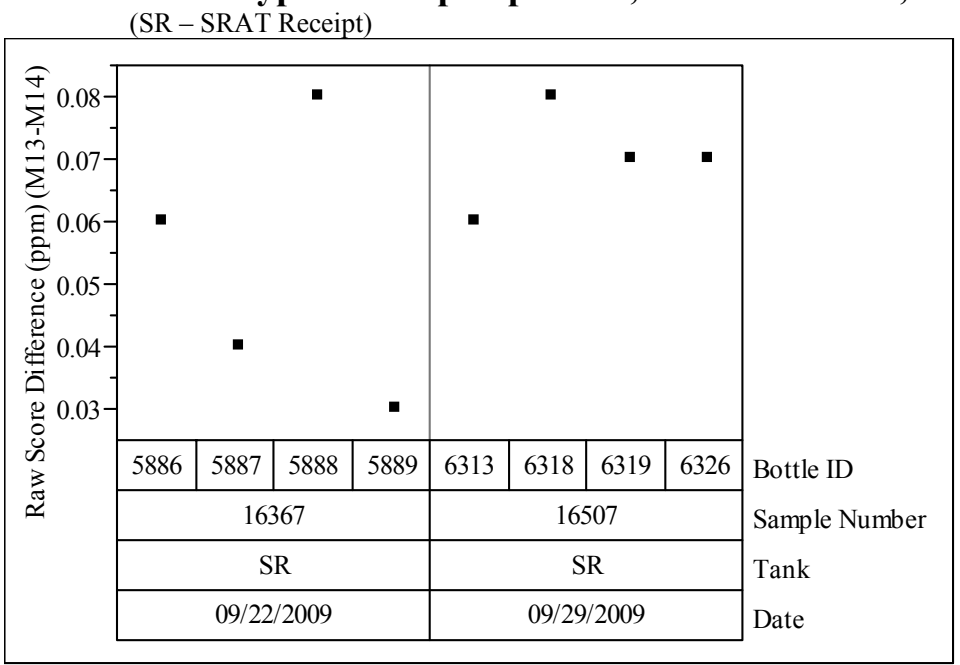

Exhibit A2e. Type of Sample=process, Anion=Sulfate; Raw Score Difference (ppm) (M13-M14) (SR - SRAT Receipt; SP - SRAT Product)

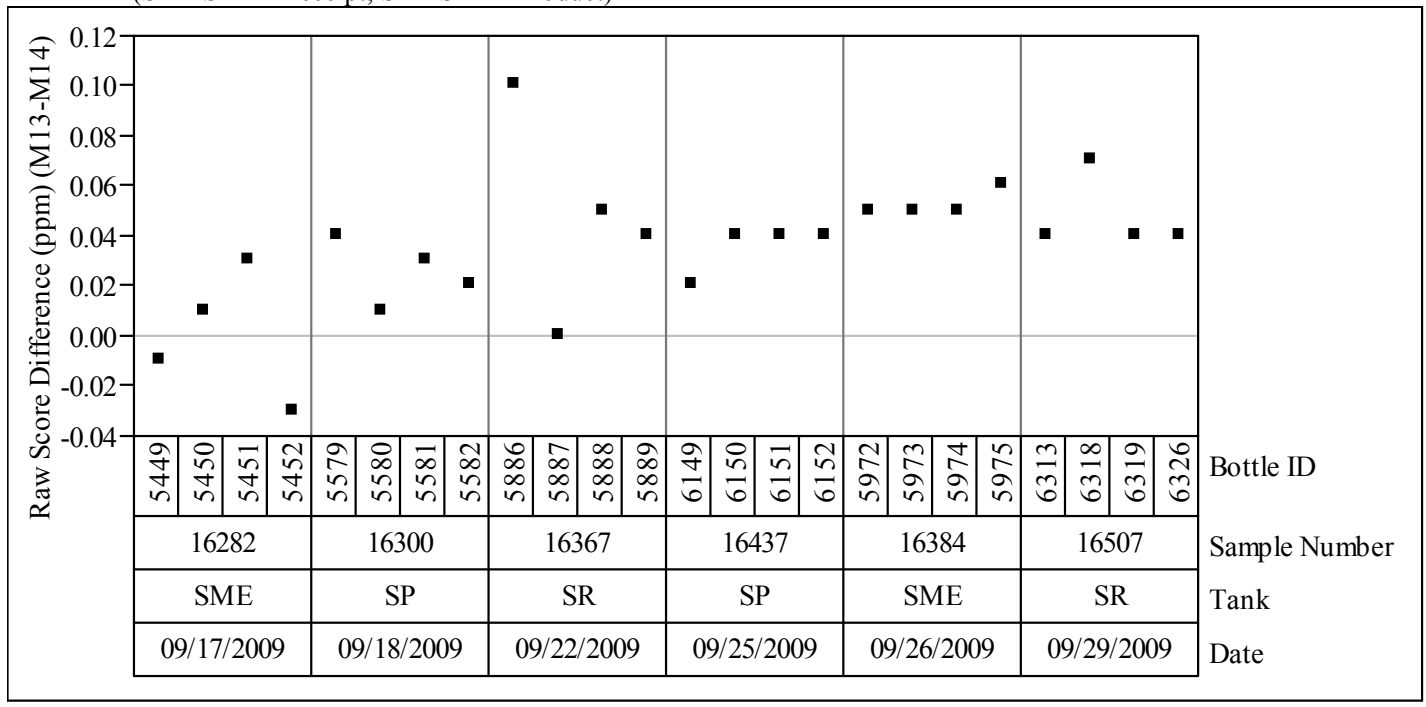


Exhibit 3a. Type=Simulated Sample Crosscheck Data 5000X Dilution, Known Concentration $(\mathrm{ppm})=1$

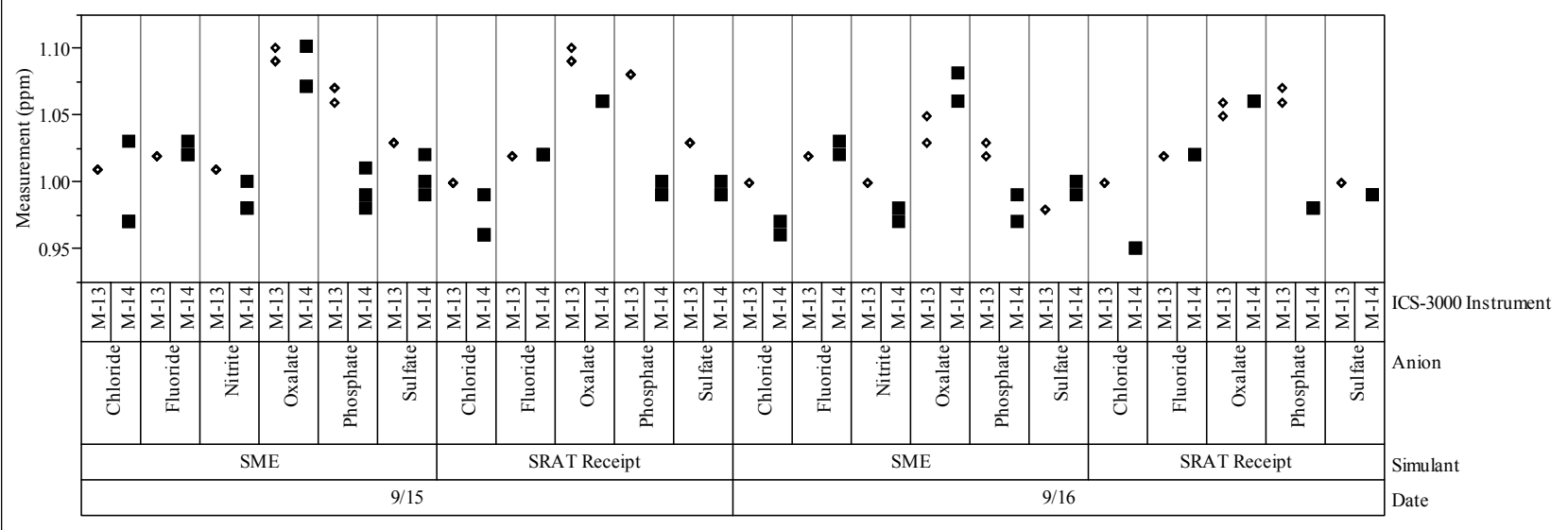

Exhibit 3b. Type=Simulated Sample Crosscheck Data 5000X Dilution, Known Concentration $(\mathrm{ppm})=1.5$

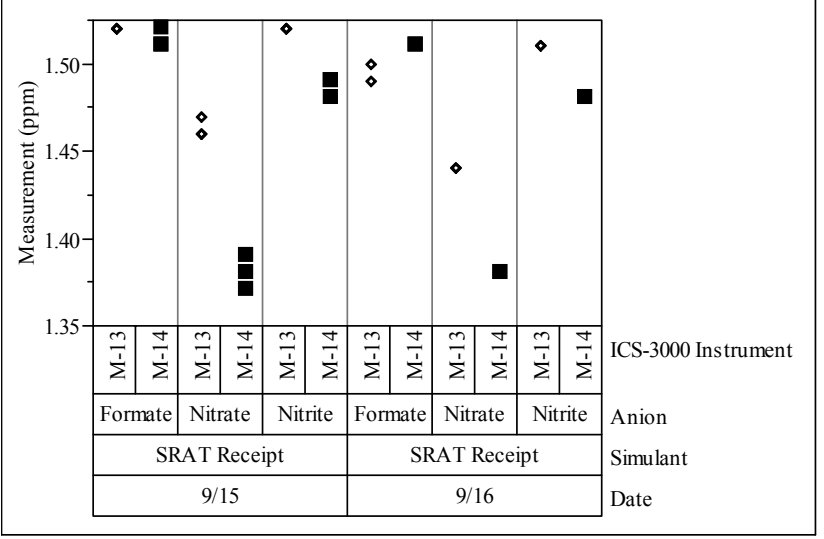

Exhibit 3c. Type $=$ Simulated Sample Crosscheck Data 5000X Dilution, Known Concentration $(\mathrm{ppm})=2.5$

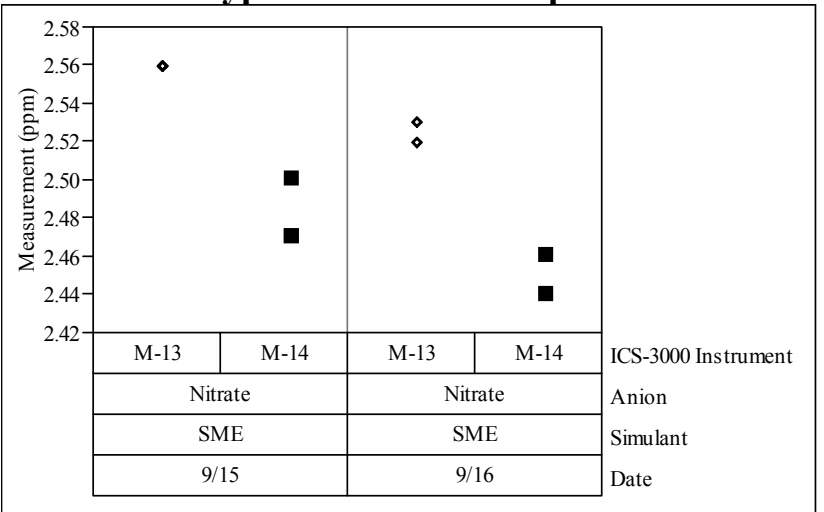


Exhibit 3d. Type=Simulated Sample Crosscheck Data 5000X Dilution, Known Concentration $(\mathrm{ppm})=6$

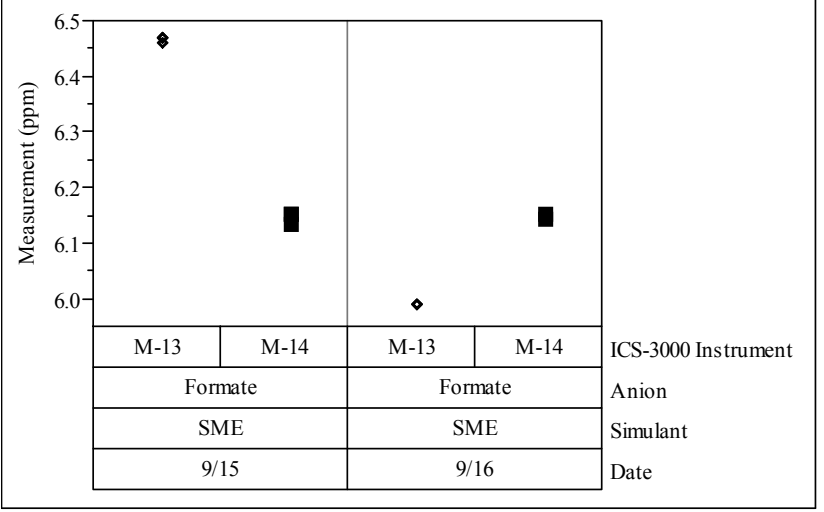

Exhibit 3e. Type=Simulated Sample Crosscheck Data 500X Dilution, Known Concentration (ppm)=1

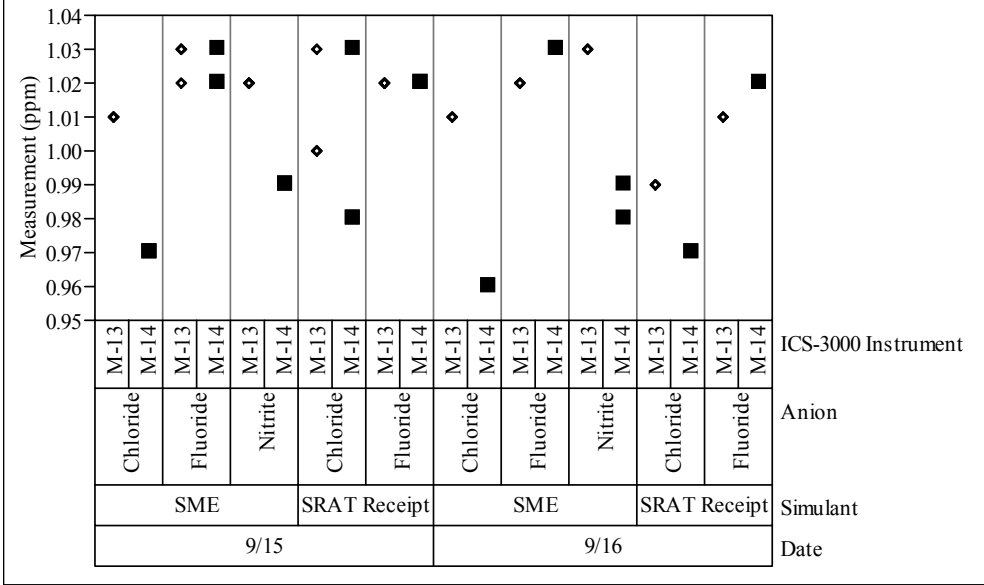

Exhibit 3f. Type=Simulated Sample Crosscheck Data 500X Dilution, Known Concentration (ppm)=2.5

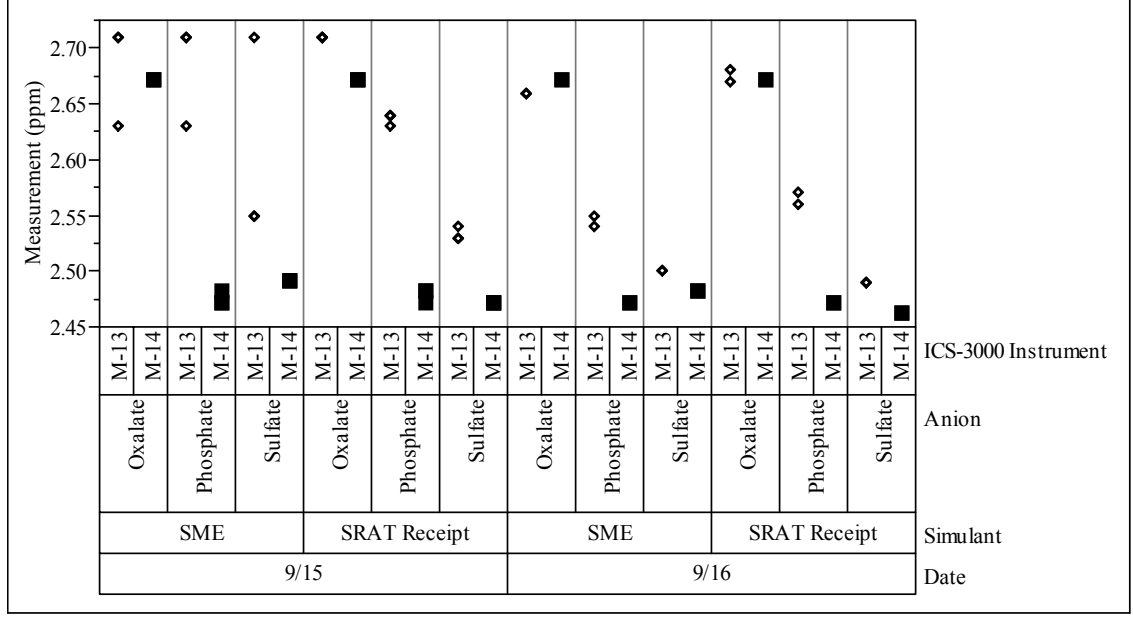


Exhibit 3g. Type=Simulated Sample Crosscheck Data 500X Dilution, Known Concentration $(\mathrm{ppm})=15$

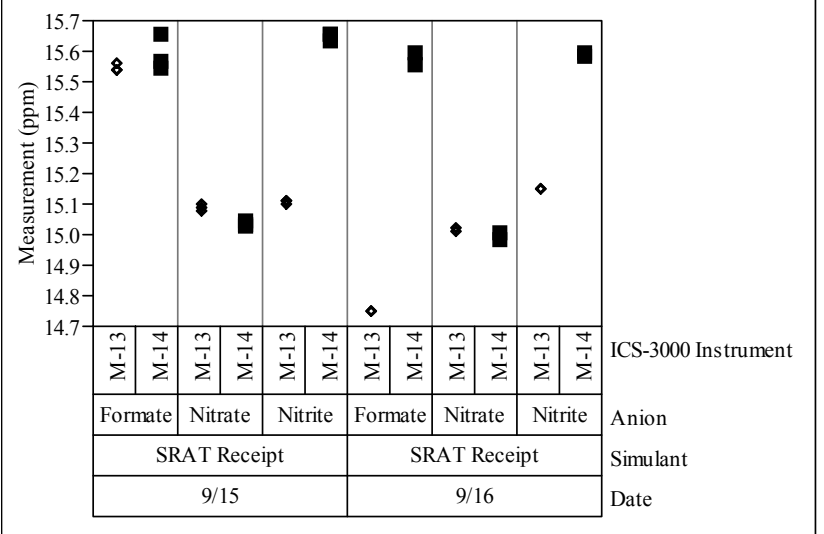

Exhibit 3h. Type=Simulated Sample Crosscheck Data 500X Dilution, Known Concentration $(\mathrm{ppm})=25$

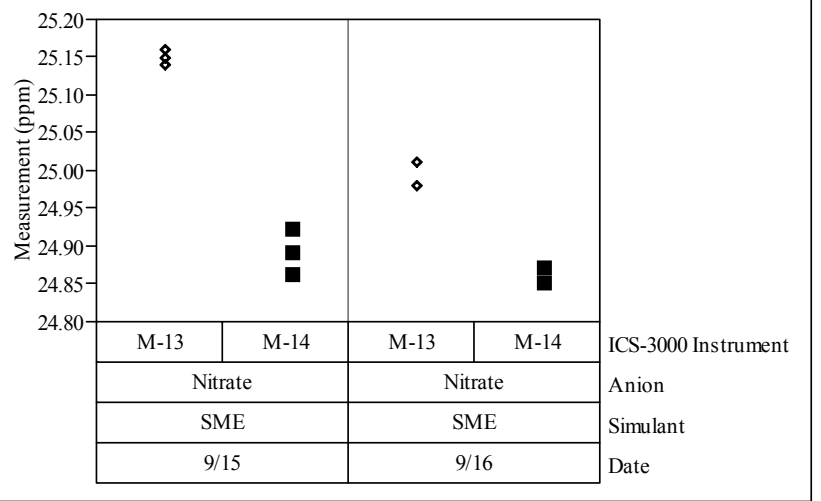

Exhibit 3i. Type=Simulated Sample Crosscheck Data 500X Dilution, Known Concentration $(\mathrm{ppm})=60$ Variability Chart for Measurement (ppm)

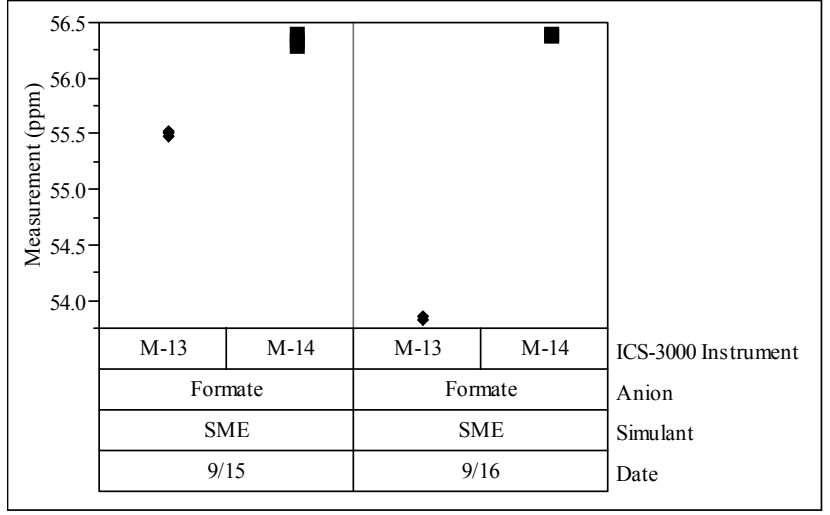


Exhibit 4a. Type=1 ppm Calibration Standard as a sample Variability Chart for Measurement (ppm)

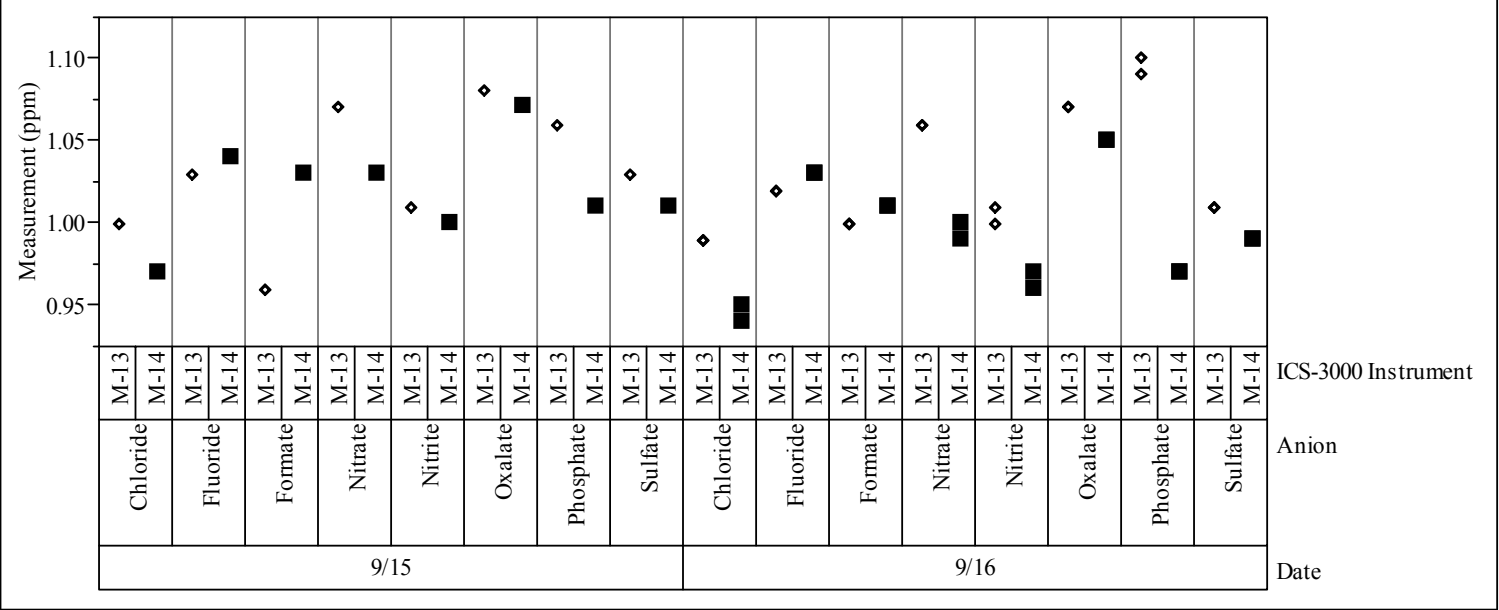

Exhibit 4b. Type $=10 \mathrm{ppm}$ Calibration Standard as a sample Variability Chart for Measurement (ppm)

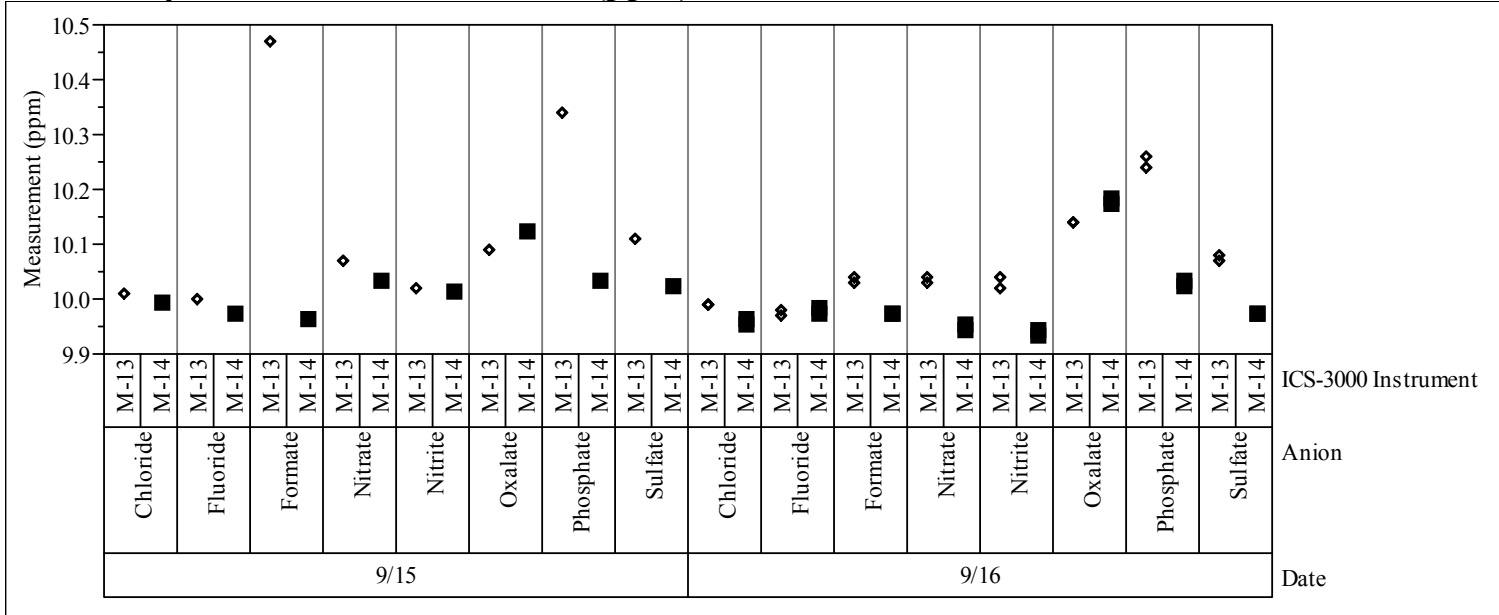

Exhibit 4c. Type $=20 \mathrm{ppm}$ Calibration Standard as a sample Variability Chart for Measurement (ppm)

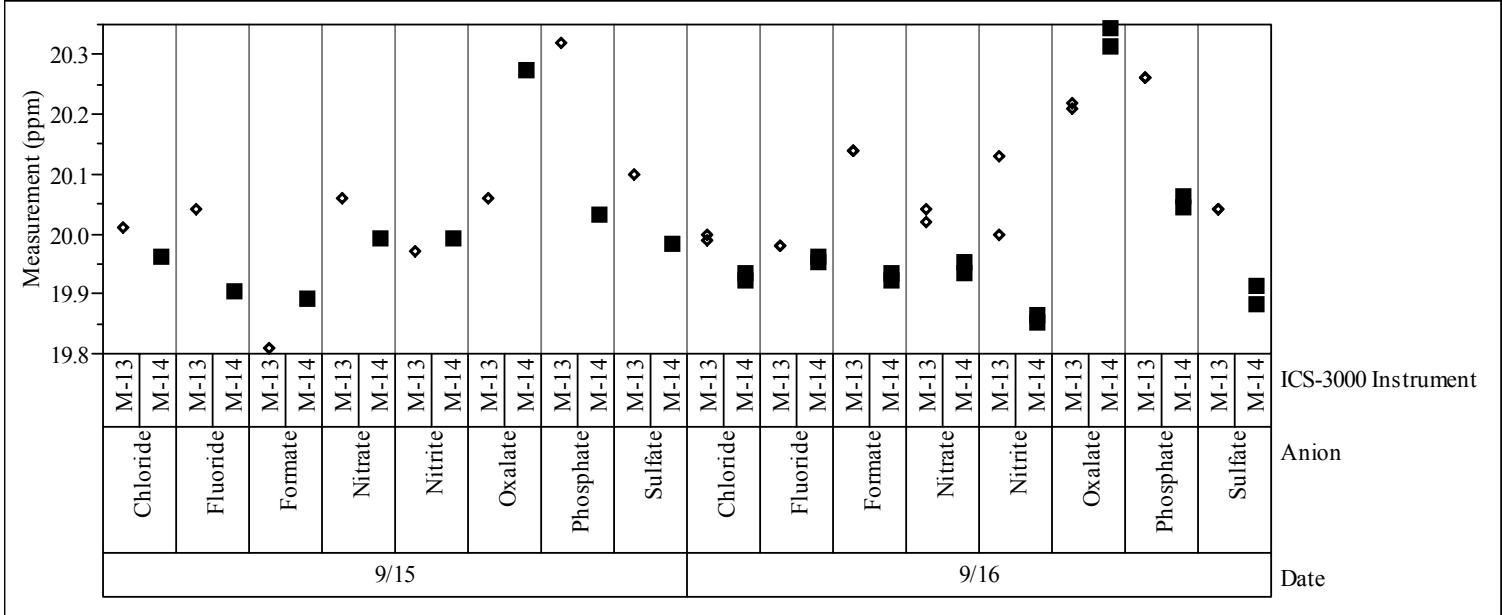


Exhibit A5. Type $=10 \mathrm{ppm}$ Standards prepared from dried salts

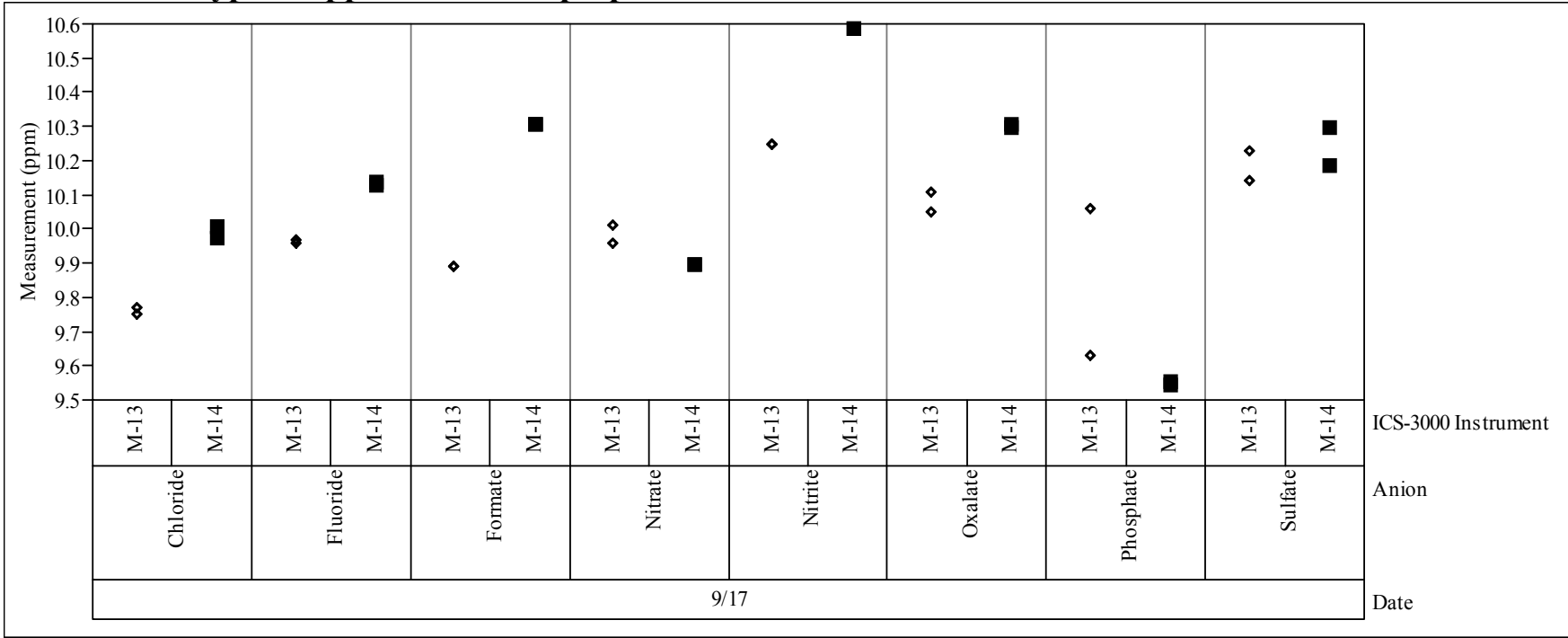


Exhibit A6a.

Oneway Analysis of Measurement (ppm) By Instrument Type of Measurement $=$ Raw, Bottle ID $=2 \mathrm{ppm}$, Anion $=$ Chloride

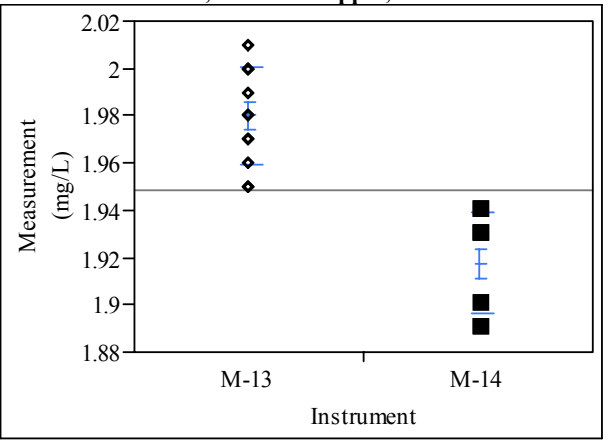

Means and Std Deviations

Level Number Mean Std Dev Std Err Mean Lower 95\% Upper 95\% $\begin{array}{lllllll}\mathrm{M}-14 & 12 & 1.91750 & 0.021373 & 0.00617 & 1.9039 & 1.9311\end{array}$

Tests that the Variances are Equal

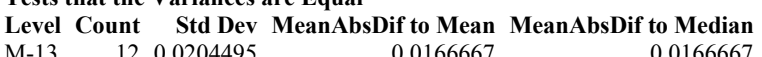

$\begin{array}{lllll}\text { M-13 } & 12 & 0.0204495 & 0.0166667 & 0.0166667 \\ \mathrm{M}-14 & 12 & 0.0213733 & 0.0195833 & 0.0175000\end{array}$

$\begin{array}{lllll}\mathrm{M}-14 & 12 & 0.0213733 & 0.0195833 & 0.0175000\end{array}$

$\begin{array}{lrrrr}\text { Test } & \text { F Ratio } & \text { DFNum } & \text { DFDen } & \text { p-Value } \\ \text { O'Brien[.5] } & 0.0750 & 1 & 22 & 0.7867 \\ \text { Brown-Forsythe } & 0.0204 & 1 & 22 & 0.8877 \\ \text { Levene } & 0.6646 & 1 & 22 & 0.4237 \\ \text { Bartlett } & 0.0205 & 1 & . & 0.8861 \\ \text { F Test 2-sided } & 1.0924 & 11 & 11 & 0.8861\end{array}$

Exhibit A6b.

Oneway Analysis of Measurement (ppm) By Instrument Type of Measurement $=$ Raw, Bottle ID $=\mathbf{2 p p m}$, Anion=Fluoride

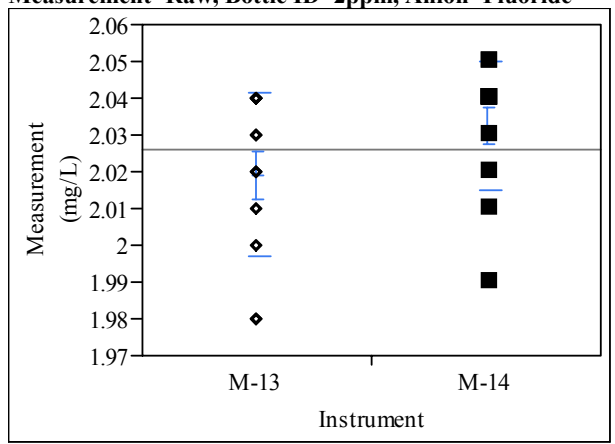

Means and Std Deviations

Level Number Mean Std Dev Std Err Mean Lower 95\% Upper 95\%

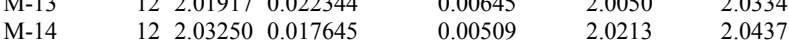

Tests that the Variances are Equal

Level Count Std Dev MeanAbsDif to Mean MeanAbsDif to Median

$\begin{array}{lrrrr}\text { M-13 } & 12 & 0.0223437 & 0.0177778 & 0.0175000\end{array}$

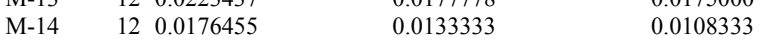

$\begin{array}{lrrrr}\text { Test } & \text { F Ratio } & \text { DFNum } & \text { DFDen } & \text { p-Value } \\ \text { O'Brien[.5] } & 0.6031 & 1 & 22 & 0.4457 \\ \text { Brown-Forsythe } & 1.1932 & 1 & 22 & 0.2865 \\ \text { Levene } & 0.8718 & 1 & 22 & 0.3606 \\ \text { Bartlett } & 0.5810 & 1 & . & 0.4459 \\ \text { F Test 2-sided } & 1.6034 & 11 & 11 & 0.4461\end{array}$

Exhibit A6c.

Oneway Analysis of Measurement (ppm) By Instrument Type of Measurement $=$ Raw, Bottle $\mathbf{I D}=\mathbf{2} \mathrm{ppm}$, Anion=Formate

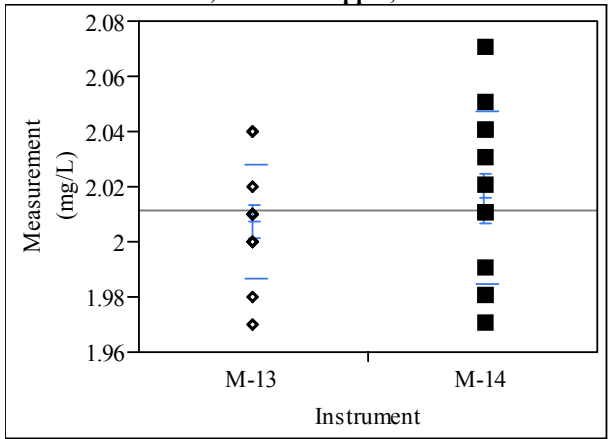

Means and Std Deviations

Level Number Mean Std Dev Std Err Mean Lower 95\% Upper 95\%

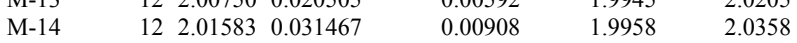

Tests that the Variances are Equal

Level Count Std Dev MeanAbsDif to Mean MeanAbsDif to Median

$\begin{array}{lllll}\text { M-13 } & 12 & 0.0205050 & 0.0145833 & 0.0141667\end{array}$

$\begin{array}{lllll}M-14 & 12 & 0.0314667 & 0.0258333 & 0.0258333\end{array}$

$\begin{array}{lrrrr}\text { Test } & \text { F Ratio } & \text { DFNum } & \text { DFDen } & \text { p-Value } \\ \text { O'Brien[.5] } & 2.7213 & 1 & 22 & 0.1132 \\ \text { Brown-Forsythe } & 3.4662 & 1 & 22 & 0.0760 \\ \text { Levene } & 3.3707 & 1 & 22 & 0.0799 \\ \text { Bartlett } & 1.8735 & 1 & . & 0.1711 \\ \text { F Test 2-sided } & 2.3550 & 11 & 11 & 0.1711\end{array}$

Exhibit A6d.

Oneway Analysis of Measurement (ppm) By Instrument Type of

Measurement $=$ Raw, Bottle ID $=2 \mathrm{ppm}$, Anion=Nitrate

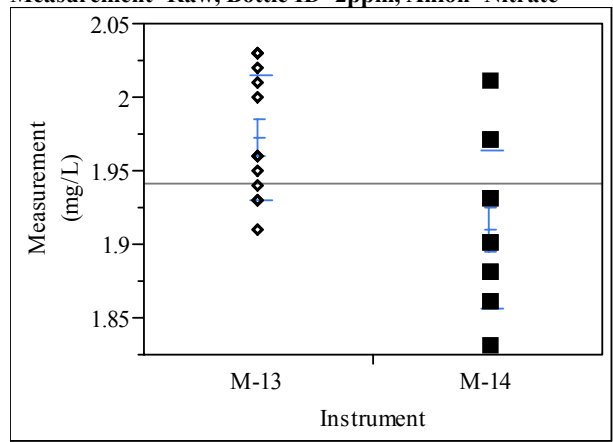

Means and Std Deviations

Level Number Mean Std Dev Std Err Mean Lower 95\% Upper 95\%

$\begin{array}{llllllr}\text { M-13 } & 12 & 1.97250 & 0.043091 & 0.01244 & 1.9451 & 1.9999\end{array}$

$\begin{array}{lllllll}\mathrm{M}-14 & 12 & 1.91000 & 0.053428 & 0.01542 & 1.8761 & 1.9439\end{array}$

Tests that the Variances are Equal

Level Count Std Dev MeanAbsDif to Mean MeanAbsDif to Median

$\begin{array}{lrrrr}\text { M-13 } & 12 & 0.0430908 & 0.0379167 & 0.0358333\end{array}$

$\begin{array}{lllll}\text { M-14 } & 12 & 0.0534279 & 0.0433333 & 0.0416667\end{array}$

$\begin{array}{lrrrr}\text { Test } & \text { F Ratio } & \text { DFNum } & \text { DFDen } & \text { p-Value } \\ \text { O'Brien[.5] } & 0.8551 & 1 & 22 & 0.3651 \\ \text { Brown-Forsythe } & 0.2407 & 1 & 22 & 0.6285 \\ \text { Levene } & 0.3217 & 1 & 22 & 0.5763 \\ \text { Bartlett } & 0.4828 & 1 & . & 0.4872 \\ \text { F Test 2-sided } & 1.5373 & 11 & 11 & 0.4873\end{array}$


Exhibit A6e.

Oneway Analysis of Measurement (ppm) By Instrument Type of Measurement $=$ Raw, Bottle ID $=2 \mathrm{ppm}$, Anion=Nitrite

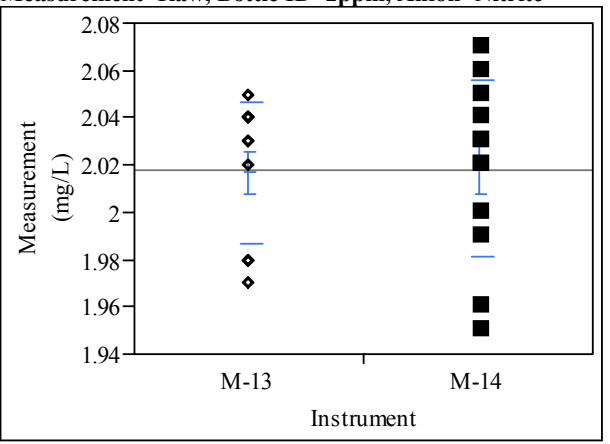

Means and Std Deviations

Level Number Mean Std Dev Std Err Mean Lower 95\% Upper 95\% $\begin{array}{llllrrr}\text { M-13 } & 12 & 2.01667 & 0.029949 & 0.00865 & 1.9976 & 2.0357\end{array}$

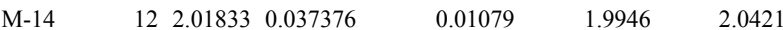

Tests that the Variances are Equal

Level Count Std Dev MeanAbsDif to Mean MeanAbsDif to Median $\begin{array}{lllll}\text { M-13 } & 12 & 0.0299495 & 0.0261111 & 0.0233333\end{array}$ $\begin{array}{lllll}\text { M-14 } & 12 & 0.0373761 & 0.0288889 & 0.0283333\end{array}$

$\begin{array}{lrrrr}\text { Test } & \text { F Ratio } & \text { DFNum } & \text { DFDen } & \text { p-Value } \\ \text { O'Brien[.5] } & 0.8273 & 1 & 22 & 0.3729 \\ \text { Brown-Forsythe } & 0.2812 & 1 & 22 & 0.6012 \\ \text { Levene } & 0.1447 & 1 & 22 & 0.7073 \\ \text { Bartlett } & 0.5121 & 1 & . & 0.4742 \\ \text { F Test 2-sided } & 1.5574 & 11 & 11 & 0.4744\end{array}$

Exhibit A6f.

Oneway Analysis of Measurement (ppm) By Instrument Type of Measurement=Raw, Bottle ID=2ppm, Anion=Oxalate

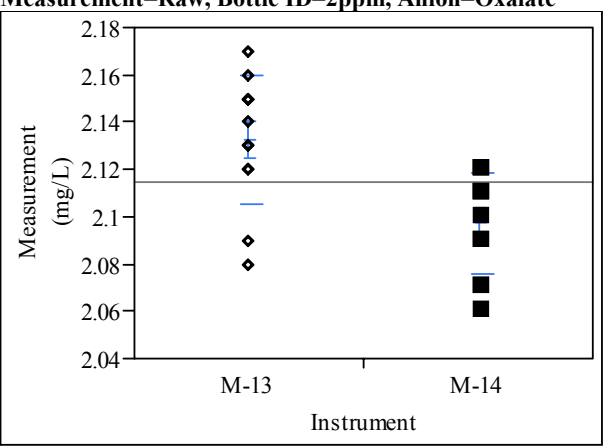

Means and Std Deviations

Level Number Mean Std Dev Std Err Mean Lower 95\% Upper 95\% $\begin{array}{llllrrr}\text { M-13 } & 12 & 2.13250 & 0.027010 & 0.00780 & 2.1153 & 2.1497\end{array}$ M-14 $12 \quad 2.09750 \quad 0.021373$

Tests that the Variances are Equal

Level Count Std Dev MeanAbsDif to Mean MeanAbsDif to Median $\begin{array}{lllll}\text { M-13 } & 12 & 0.0270101 & 0.0208333 & 0.0208333\end{array}$ $\begin{array}{lllll}\mathrm{M}-14 & 12 & 0.0213733 & 0.0179167 & 0.0175000\end{array}$

$\begin{array}{lrrrr}\text { Test } & \text { F Ratio } & \text { DFNum } & \text { DFDen } & \text { p-Value } \\ \text { O'Brien[.5] } & 0.7307 & 1 & 22 & 0.4019 \\ \text { Brown-Forsythe } & 0.2983 & 1 & 22 & 0.5904 \\ \text { Levene } & 0.2815 & 1 & 22 & 0.6011 \\ \text { Bartlett } & 0.5713 & 1 & . & 0.4498 \\ \text { F Test 2-sided } & 1.5970 & 11 & 11 & 0.4499\end{array}$

\section{Exhibit A6g.}

Oneway Analysis of Measurement (ppm) By Instrument Type of Measurement $=$ Raw, Bottle ID $=2 \mathrm{ppm}$, Anion=Phosphate

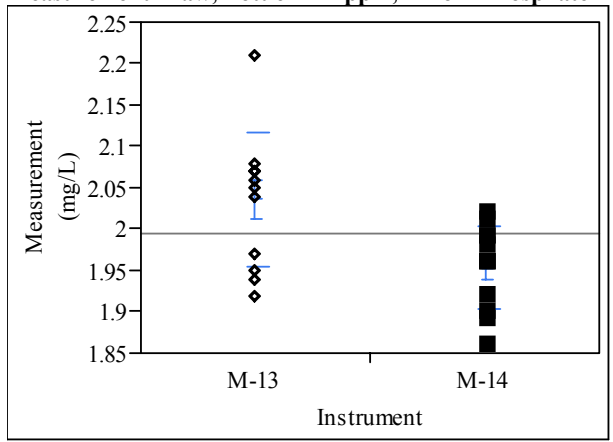

Means and Std Deviations

Level Number Mean Std Dev Std Err Mean Lower 95\% Upper 95\% $\begin{array}{lrrrrrr}\text { M-13 } & 12 & 2.03583 & 0.080279 & 0.02317 & 1.9848 & 2.0868 \\ \text { M-14 } & 12 & 1.95333 & 0.050332 & 0.01453 & 1.9214 & 1.9853\end{array}$

Tests that the Variances are Equal

Level Count Std Dev MeanAbsDif to Mean MeanAbsDif to Median

$\begin{array}{lllll}\text { M-13 } & 12 & 0.0802789 & 0.0605556 & 0.0575000\end{array}$

$\begin{array}{lllll}\mathrm{M}-14 & 12 & 0.0503322 & 0.0405556 & 0.0383333\end{array}$

$\begin{array}{lrrrr}\text { Test } & \text { F Ratio } & \text { DFNum } & \text { DFDen } & \text { p-Value } \\ \text { O'Brien[.5] } & 1.6769 & 1 & 22 & 0.2088 \\ \text { Brown-Forsythe } & 1.0453 & 1 & 22 & 0.3177 \\ \text { Levene } & 1.5078 & 1 & 22 & 0.2324 \\ \text { Bartlett } & 2.2145 & 1 & . & 0.1367 \\ \text { F Test 2-sided } & 2.5440 & 11 & 11 & 0.1367\end{array}$

\section{Exhibit A6h.}

Oneway Analysis of Measurement (ppm) By Instrument Type of Measurement=Raw, Bottle ID $=2$ ppm, Anion $=$ Sulfate

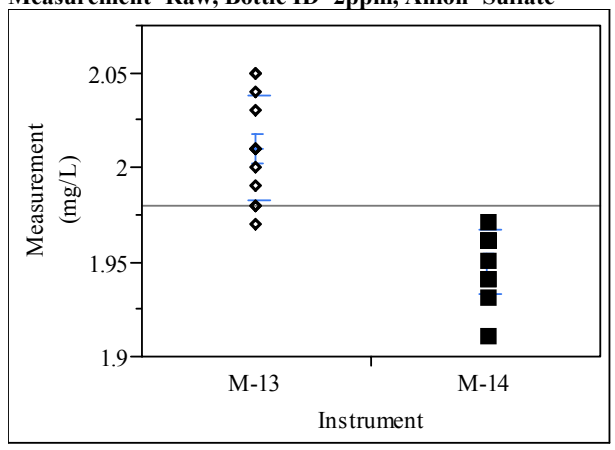

Means and Std Deviations

Level Number Mean Std Dev Std Err Mean Lower 95\% Upper 95\% $\begin{array}{lrrrrrr}\text { M-13 } & 12 & 2.01000 & 0.027634 & 0.00798 & 1.9924 & 2.0276 \\ \text { M-14 } & 12 & 1.95000 & 0.017056 & 0.00492 & 1.9392 & 1.9608\end{array}$

Tests that the Variances are Equal

Level Count Std Dev MeanAbsDif to Mean MeanAbsDif to Median

$\begin{array}{lllll}\text { M-13 } & 12 & 0.0276340 & 0.0216667 & 0.0216667\end{array}$

$\begin{array}{lllll}\mathrm{M}-14 & 12 & 0.0170561 & 0.0133333 & 0.0116667\end{array}$

$\begin{array}{lrrrr}\text { Test } & \text { F Ratio } & \text { DFNum } & \text { DFDen } & \text { p-Value } \\ \text { O'Brien[.5] } & 3.3723 & 1 & 22 & 0.0799 \\ \text { Brown-Forsythe } & 2.3855 & 1 & 22 & 0.1367 \\ \text { Levene } & 2.3913 & 1 & 22 & 0.1363 \\ \text { Bartlett } & 2.3604 & 1 & . & 0.1245 \\ \text { F Test 2-sided } & 2.6250 & 11 & 11 & 0.1245\end{array}$


Exhibit A7a.

Oneway Analysis of Measurement (ppm) By Instrument Type of Measurement $=$ Raw, Bottle ID=16ppm, Anion=Chloride

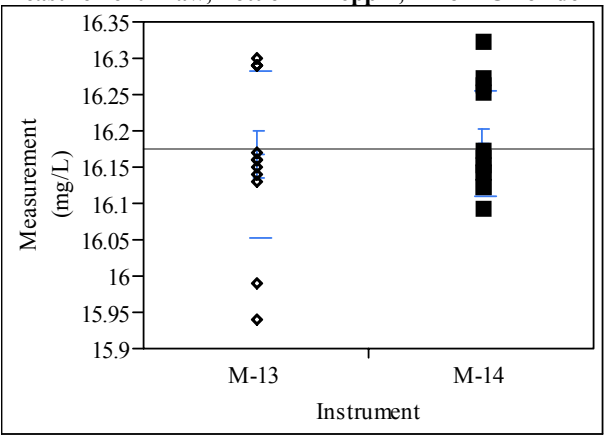

Means and Std Deviations

Level Number Mean Std Dev Std Err Mean Lower 95\% Upper 95\% $1216.16750 .116160-0.03353 \quad 16.094$ $12 \quad 16.1675 \quad 0.116160$ $0.03353 \quad 16.094$ 16.241 M-14 $\begin{array}{lll}12 & 16.1825 & 0.073128\end{array}$ 0.02111 16.136 16.229

Tests that the Variances are Equal

Level Count Std Dev MeanAbsDif to Mean MeanAbsDif to Median

$\begin{array}{lrrrr}\text { M-13 } & 12 & 0.1161602 & 0.0837500 & 0.0825000\end{array}$

$\begin{array}{lllll}\mathrm{M}-14 & 12 & 0.0731282 & 0.0616667 & 0.0558333\end{array}$

$\begin{array}{lrrrr}\text { Test } & \text { F Ratio } & \text { DFNum } & \text { DFDen } & \text { p-Value } \\ \text { O'Brien[.5] } & 2.1407 & 1 & 22 & 0.1576 \\ \text { Brown-Forsythe } & 0.9586 & 1 & 22 & 0.3382 \\ \text { Levene } & 0.8312 & 1 & 22 & 0.3718 \\ \text { Bartlett } & 2.1771 & 1 & . & 0.1401 \\ \text { F Test 2-sided } & 2.5232 & 11 & 11 & 0.1401\end{array}$

\section{Exhibit A7b.}

Oneway Analysis of Measurement (ppm) By Instrument Type of

Measurement $=$ Raw, Bottle ID $=16 \mathrm{ppm}$, Anion=Fluoride

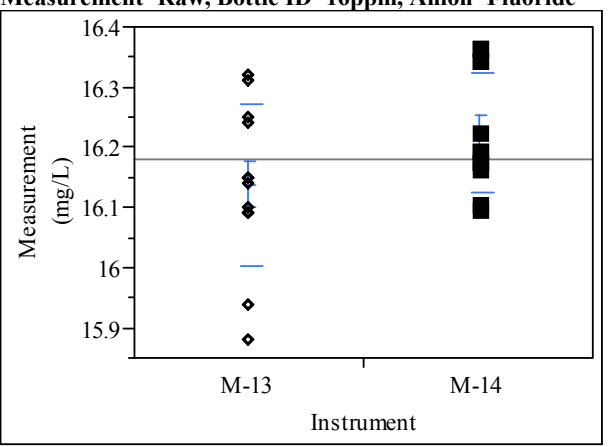

Means and Std Deviations

Level Number Mean Std Dev Std Err Mean Lower 95\% Upper 95\% $\begin{array}{lllllll}\text { M-13 } & 12 & 16.1383 & 0.134085 & 0.03871 & 16.053 & 16.224 \\ \text { M-14 } & 12 & 16.2242 & 0.099769 & 0.02880 & 16.161 & 16.288\end{array}$

Tests that the Variances are Equal

Level Count Std Dev MeanAbsDif to Mean MeanAbsDif to Median

$\begin{array}{lllll}\text { M-13 } & 12 & 0.1340850 & 0.0986111 & 0.0983333\end{array}$

$\begin{array}{lllll}\mathrm{M}-14 & 12 & 0.0997687 & 0.0838889 & 0.0775000\end{array}$

$\begin{array}{lrrrr}\text { Test } & \text { F Ratio } & \text { DFNum } & \text { DFDen } & \text { p-Value } \\ \text { O'Brien[.5] } & 1.1538 & 1 & 22 & 0.2944 \\ \text { Brown-Forsythe } & 0.4285 & 1 & 22 & 0.5195 \\ \text { Levene } & 0.2696 & 1 & 22 & 0.6088 \\ \text { Bartlett } & 0.9064 & 1 & . & 0.3411 \\ \text { F Test 2-sided } & 1.8062 & 11 & 11 & 0.3412\end{array}$

Exhibit A7c.

Oneway Analysis of Measurement (ppm) By Instrument Type of Measurement=Raw, Bottle ID=16ppm, Anion=Formate

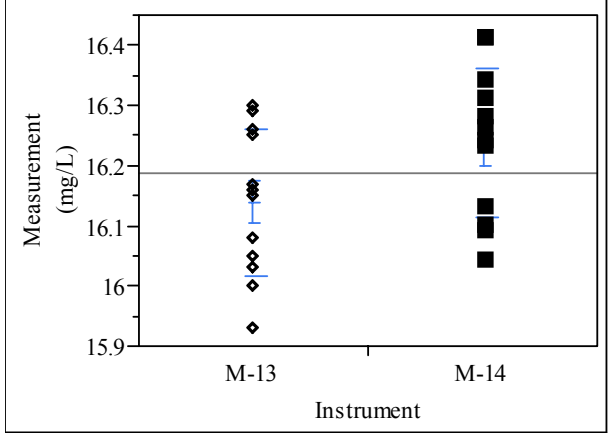

Means and Std Deviations

Level Number Mean Std Dev Std Err Mean Lower 95\% Upper 95\% $\begin{array}{lrrrrrr}\text { M-13 } & 12 & 16.1392 & 0.121913 & 0.03519 & 16.062 & 16.217\end{array}$ $\begin{array}{lllllll}\text { M-14 } & 12 & 16.2367 & 0.123901 & 0.03577 & 16.158 & 16.315\end{array}$

Tests that the Variances are Equal

Level Count Std Dev MeanAbsDif to Mean MeanAbsDif to Median

$\begin{array}{lllll}\text { M-13 } & 12 & 0.1219134 & 0.1009722 & 0.0991667\end{array}$

$\begin{array}{lllll}\mathrm{M}-14 & 12 & 0.1239012 & 0.0988889 & 0.0983333\end{array}$

$\begin{array}{lrrrr}\text { Test } & \text { F Ratio } & \text { DFNum } & \text { DFDen } & \text { p-Value } \\ \text { O'Brien[.5] } & 0.0062 & 1 & 22 & 0.9377 \\ \text { Brown-Forsythe } & 0.0009 & 1 & 22 & 0.9765 \\ \text { Levene } & 0.0062 & 1 & 22 & 0.9380 \\ \text { Bartlett } & 0.0028 & 1 & . & 0.9582 \\ \text { F Test 2-sided } & 1.0329 & 11 & 11 & 0.9582\end{array}$

\section{Exhibit A7d.}

Oneway Analysis of Measurement (ppm) By Instrument Type of Measurement=Raw, Bottle ID=16ppm, Anion=Nitrate

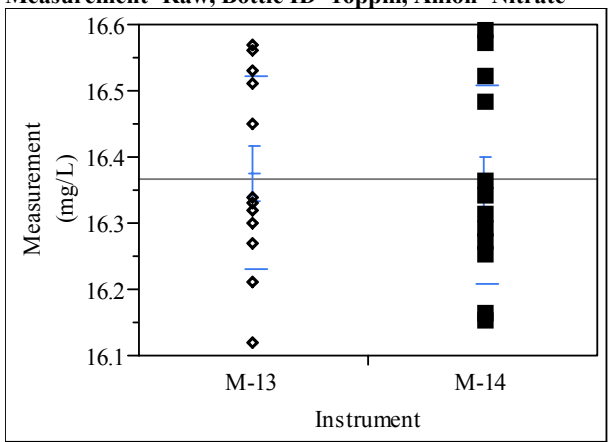

Means and Std Deviations

Level Number Mean Std Dev Std Err Mean Lower 95\% Upper 95\% $\begin{array}{lllllrr}\text { M-13 } & 12 & 16.3758 & 0.146254 & 0.04222 & 16.283 & 16.469\end{array}$ $\begin{array}{lllllll}\text { M-14 } & 12 & 16.3575 & 0.150461 & 0.04343 & 16.262 & 16.453\end{array}$

Tests that the Variances are Equal

Level Count Std Dev MeanAbsDif to Mean MeanAbsDif to Median $\begin{array}{lllll}\text { M-13 } & 12 & 0.1462537 & 0.1234722 & 0.1175000\end{array}$ $\begin{array}{lllll}\mathrm{M}-14 & 12 & 0.1504614 & 0.1220833 & 0.1191667\end{array}$

$\begin{array}{lrrrr}\text { Test } & \text { F Ratio } & \text { DFNum } & \text { DFDen } & \text { p-Value } \\ \text { O'Brien[.5] } & 0.0187 & 1 & 22 & 0.8926 \\ \text { Brown-Forsythe } & 0.0020 & 1 & 22 & 0.9645 \\ \text { Levene } & 0.0021 & 1 & 22 & 0.9640 \\ \text { Bartlett } & 0.0085 & 1 & . & 0.9267 \\ \text { F Test 2-sided } & 1.0584 & 11 & 11 & 0.9267\end{array}$


Exhibit A7e.

Oneway Analysis of Measurement (ppm) By Instrument Type of Measurement $=$ Raw, Bottle ID $=16 \mathrm{ppm}$, Anion=Nitrite

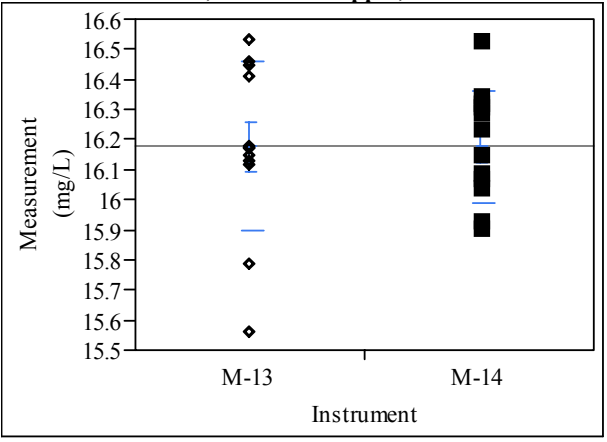

Means and Std Deviations

Level Number Mean Std Dev Std Err Mean Lower 95\% Upper 95\% $\begin{array}{lllllrr}\text { M-13 } & 12 & 16.1767 & 0.281274 & 0.08120 & 15.998 & 16.355\end{array}$ $\begin{array}{lllllll}\text { M-14 } & 12 & 16.1758 & 0.185691 & 0.05360 & 16.058 & 16.294\end{array}$

Tests that the Variances are Equal

Level Count Std Dev MeanAbsDif to Mean MeanAbsDif to Median

$\begin{array}{lllll}\text { M-13 } & 12 & 0.2812742 & 0.1911111 & 0.1900000\end{array}$

$\begin{array}{lllll}\text { M-14 } & 12 & 0.1856908 & 0.1541667 & 0.1541667\end{array}$

$\begin{array}{lrrrr}\text { Test } & \text { F Ratio } & \text { DFNum } & \text { DFDen } & \text { p-Value } \\ \text { O'Brien[.5] } & 1.3479 & 1 & 22 & 0.2581 \\ \text { Brown-Forsythe } & 0.3182 & 1 & 22 & 0.5784 \\ \text { Levene } & 0.3425 & 1 & 22 & 0.5644 \\ \text { Bartlett } & 1.7644 & 1 & . & 0.1841 \\ \text { F Test 2-sided } & 2.2945 & 11 & 11 & 0.1841\end{array}$

Exhibit A7f.

Oneway Analysis of Measurement (ppm) By Instrument Type of Measurement=Raw, Bottle ID=16ppm, Anion=Oxalate

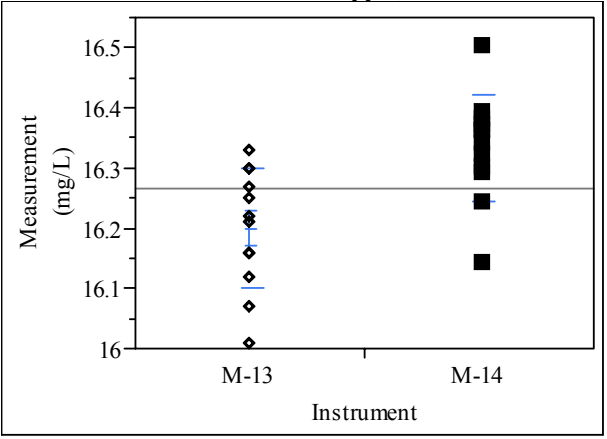

Means and Std Deviations

Level Number Mean Std Dev Std Err Mean Lower 95\% Upper 95\% $\begin{array}{lllllrr}\text { M-13 } & 12 & 16.2000 & 0.098811 & 0.02852 & 16.137 & 16.263\end{array}$ $\begin{array}{lllllll}\text { M-14 } & 12 & 16.3325 & 0.088021 & 0.02541 & 16.277 & 16.388\end{array}$

Tests that the Variances are Equal

Level Count Std Dev MeanAbsDif to Mean MeanAbsDif to Median $\begin{array}{lllll}\text { M-13 } & 12 & 0.0988111 & 0.0800000 & 0.0783333\end{array}$ $\begin{array}{lllll}\mathrm{M}-14 & 12 & 0.0880212 & 0.0620833 & 0.0608333\end{array}$

$\begin{array}{lrrrr}\text { Test } & \text { F Ratio } & \text { DFNum } & \text { DFDen } & \text { p-Value } \\ \text { O'Brien[.5] } & 0.1439 & 1 & 22 & 0.7081 \\ \text { Brown-Forsythe } & 0.4993 & 1 & 22 & 0.4872 \\ \text { Levene } & 0.6090 & 1 & 22 & 0.4435 \\ \text { Bartlett } & 0.1404 & 1 & . & 0.7079 \\ \text { F Test 2-sided } & 1.2602 & 11 & 11 & 0.7080\end{array}$

\section{Exhibit A7g.}

Oneway Analysis of Measurement (ppm) By Instrument Type of Measurement=Raw, Bottle ID=16ppm, Anion=Phosphate

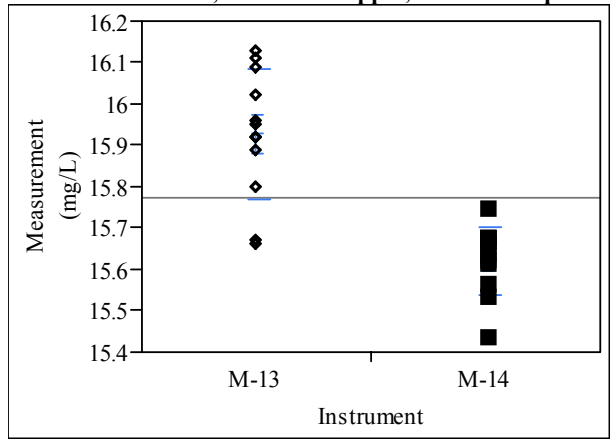

Means and Std Deviations

Level Number Mean Std Dev Std Err Mean Lower 95\% Upper 95\% $\begin{array}{lrrrrrr}\text { M-13 } & 12 & 15.9267 & 0.155641 & 0.04493 & 15.828 & 16.026\end{array}$ $\begin{array}{lllllll}\text { M-14 } & 12 & 15.6199 & 0.081198 & 0.02344 & 15.568 & 15.671\end{array}$

Tests that the Variances are Equal

Level Count Std Dev MeanAbsDif to Mean MeanAbsDif to Median

$\begin{array}{lllll}\text { M-13 } & 12 & 0.1556414 & 0.1166667 & 0.1166667\end{array}$

$\begin{array}{lllll}\mathrm{M}-14 & 12 & 0.0811984 & 0.0584667 & 0.0567667\end{array}$

$\begin{array}{lrrrr}\text { Test } & \text { F Ratio } & \text { DFNum } & \text { DFDen } & \text { p-Value } \\ \text { O'Brien[.5] } & 3.6626 & 1 & 22 & 0.0687 \\ \text { Brown-Forsythe } & 3.3197 & 1 & 22 & 0.0821 \\ \text { Levene } & 3.3209 & 1 & 22 & 0.0820 \\ \text { Bartlett } & 4.1716 & 1 & . & 0.0411 \\ \text { F Test 2-sided } & 3.6741 & 11 & 11 & 0.0410\end{array}$

Exhibit A7h.

Oneway Analysis of Measurement (ppm) By Instrument Type of Measurement $=$ Raw, Bottle ID $=16$ ppm, Anion=Sulfate

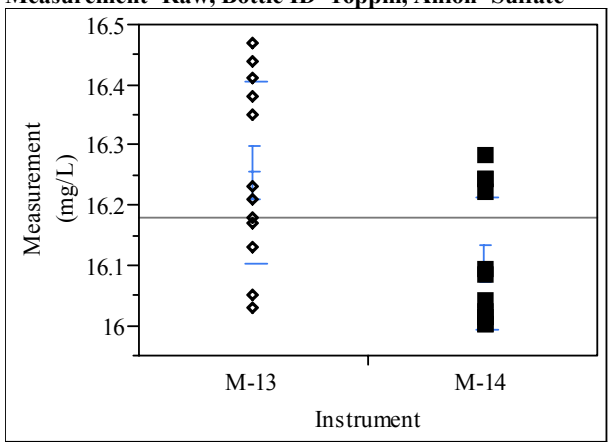

Means and Std Deviations

Level Number Mean Std Dev Std Err Mean Lower 95\% Upper 95\% $\begin{array}{lllllrr}\text { M-13 } & 12 & 16.2542 & 0.151625 & 0.04377 & 16.158 & 16.351\end{array}$ $\begin{array}{lllllll}\text { M-14 } & 12 & 16.1025 & 0.109804 & 0.03170 & 16.033 & 16.172\end{array}$

Tests that the Variances are Equal

Level Count Std Dev MeanAbsDif to Mean MeanAbsDif to Median

$\begin{array}{lllll}\text { M-13 } & 12 & 0.1516250 & 0.1298611 & 0.1258333\end{array}$

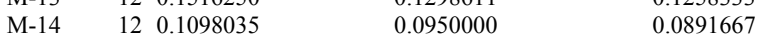

$\begin{array}{lrrrr}\text { Test } & \text { F Ratio } & \text { DFNum } & \text { DFDen } & \text { p-Value } \\ \text { O'Brien[.5] } & 2.7305 & 1 & 22 & 0.1126 \\ \text { Brown-Forsythe } & 1.3070 & 1 & 22 & 0.2652 \\ \text { Levene } & 2.1432 & 1 & 22 & 0.1573 \\ \text { Bartlett } & 1.0773 & 1 & . & 0.2993 \\ \text { F Test 2-sided } & 1.9068 & 11 & 11 & 0.2994\end{array}$


Exhibit A8a.

Distributions Anion=Chloride Raw Score Difference (ppm) (M13-M14)

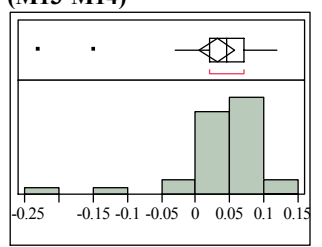

Quantiles

$100.0 \%$ maximum 0.1200

$99.5 \%$

$97.5 \%$

$90.0 \%$

$75.0 \%$

$50.0 \%$ median 0.0450

$25.0 \%$ quartile $\quad 0.0200$

$10.0 \% \quad-0.0290$

$2.5 \% \quad-0.2300$

$0.5 \% \quad-0.2300$

$0.0 \%$ minimum -0.2300

Moments

Mean $\quad 0.0316667$

Std Dev $\quad 0.0702745$

Std Err Mean $\quad 0.0128303$

upper $95 \%$ Mean 0.0579076

lower $95 \%$ Mean 0.0054257

Exhibit A8b.

Distributions Anion=Fluoride

Raw Score Difference (ppm) (M13-M14)

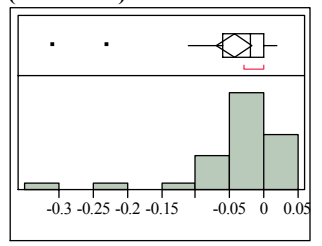

Quantiles

$100.0 \%$ maximum 0.0200

$99.5 \%$

$\begin{array}{ll}97.5 \% & 0.0200 \\ 90.0 \% & 0.0000\end{array}$

$75.0 \%$ quartile $\quad 0.0000$

$50.0 \%$ median -0.0200

$25.0 \%$ quartile $\quad-0.0600$

$10.0 \% \quad-0.1090$

$2.5 \% \quad-0.3100$

$0.5 \% \quad-0.3100$

$0.0 \%$ minimum -0.3100

Moments

Mean $\quad-0.043333$

Std Dev $\quad 0.0698932$

Std Err Mean $\quad 0.0127607$

upper $95 \%$ Mean -0.017235

lower 95\% Mean - 0.069432

$\mathrm{N}$
Exhibit A8c.

Distributions Anion=Formate Raw Score Difference (ppm) (M13-M14)

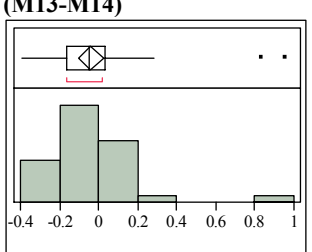

Quantiles

$100.0 \%$ maximum 0.9500

$99.5 \% \quad 0.9500$

$\begin{array}{ll}97.5 \% & 0.8990\end{array}$

$90.0 \% \quad 0.1630$

$75.0 \%$ quartile $\quad 0.0300$

$50.0 \%$ median -0.0500

$25.0 \%$ quartile $\quad-0.1600$

$10.0 \% \quad-0.3360$

$2.5 \% \quad-0.3815$

$0.5 \% \quad-0.3900$

$0.0 \%$ minimum -0.3900

Moments

Mean $\quad-0.040893$

Std Dev $\quad 0.2398581$

Std Err Mean $\quad 0.0320524$ upper 95\% Mean 0.0233416

lower 95\% Mean - 0.105127

$\mathrm{N}$

Exhibit A8d.

Distributions Anion=Nitrate Raw Score Difference (ppm) (M13-M14)

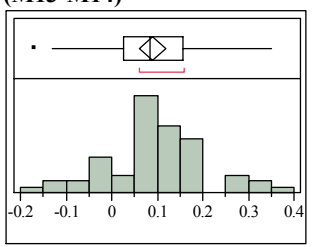

Quantiles

$100.0 \%$ maximum 0.3500

$99.5 \%$

0.3500

$\begin{array}{ll}97.5 \% & 0.3372 \\ 90.0 \% & 0.2530\end{array}$

$90.0 \% \quad 0.2530$

$75.0 \%$ quartile $\quad 0.1575$

$50.0 \%$ median 0.0850

$25.0 \%$ quartile 0.0275

$10.0 \% \quad-0.0460$

$2.5 \% \quad-0.1530$

$0.5 \% \quad-0.1700$

$0.0 \%$ minimum -0.1700

Moments

Mean $\quad 0.0891071$

Std Dev $\quad 0.1068266$

Std Err Mean $\quad 0.0142753$

upper $95 \%$ Mean 0.1177155

lower 95\% Mean 0.0604988

$\mathrm{N}$
Exhibit A8e.

Distributions Anion=Nitrite Raw Score Difference (ppm) (M13-M14)

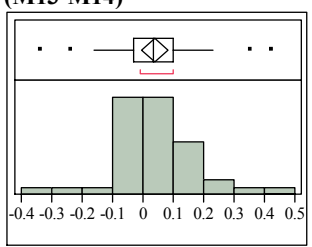

Quantiles

$100.0 \%$ maximum 0.4200

$99.5 \%$

$97.5 \%$

$90.0 \%$

$75.0 \%$ quartile

$50.0 \%$ median

$25.0 \%$ quartile

$10.0 \%$

$2.5 \%$

$0.5 \%$

Moments

Mean

Std Dev

Std Err Mean

0.4200

0.4183

0.2240

0.1000

0.0350

$-0.0300$

$-0.0960$

$-0.3400$

upper $95 \%$ Mean $\quad 0.083924$

lower 95\% Mean - 0.003424

Exhibit A8f.

Distributions Anion=Oxalate

Raw Score Difference (ppm) (M13-M14)

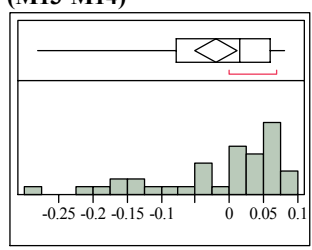

Quantiles

$100.0 \%$ maximum 0.0800

$99.5 \%$

$97.5 \%$

$90.0 \%$

$75.0 \%$ quartile

$50.0 \%$ median

$25.0 \%$ quartile

$10.0 \%$

$2.5 \%$

$0.5 \%$

$0.0 \%$ minimum -0.2800

Moments

Mean

Std Dev

$-0.019737$

0.0957894

Std Err Mean $\quad 0.0155391$

upper $95 \%$ Mean 0.0117483

lower $95 \%$ Mean -0.051222
Exhibit A8g.

Distributions Anion=Phosphate Raw Score Difference (ppm) (M13-M14)

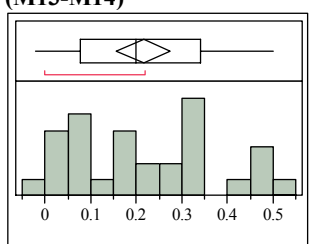

Quantiles

$100.0 \%$ maximum 0.5000

$99.5 \% \quad 0.5000$

$\begin{array}{ll}97.5 \% & 0.5000\end{array}$

$90.0 \% \quad 0.4860$

$75.0 \%$ quartile $\quad 0.3400$

$50.0 \%$ median 0.2000

$25.0 \%$ quartile $\quad 0.0775$

$10.0 \% \quad 0.0010$

$2.5 \% \quad-0.0200$

$0.5 \% \quad-0.0200$

$0.0 \%$ minimum -0.0200

Moments

Mean $\quad 0.2153733$

Std Dev $\quad 0.1615621$

Std Err Mean $\quad 0.0294971$

upper 95\% Mean 0.2757016

lower 95\% Mean 0.1550451

Exhibit A8h.

Distributions Anion=Sulfate

Raw Score Difference (ppm) (M13-M14)

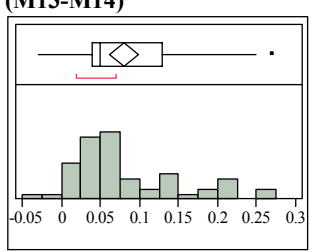

Quantiles

$100.0 \%$ maximum 0.2700

$99.5 \% \quad 0.2700$

$97.5 \% \quad 0.2625$

$90.0 \% \quad 0.2000$

$75.0 \%$ quartile $\quad 0.1300$

$50.0 \%$ median $\quad 0.0500$

$25.0 \%$ quartile $\quad 0.0400$

$10.0 \% \quad 0.0100$

$2.5 \% \quad-0.0225$

$0.5 \% \quad-0.0300$

$0.0 \%$ minimum -0.0300

Moments

Mean $\quad 0.0801852$

Std Dev $\quad 0.0689679$

Std Err Mean 0.0093853

upper 95\% Mean 0.0990098

lower 95\% Mean 0.0613606

$\mathrm{N}$ 
Exhibit A8i.

Distributions Anion=Chloride, Type of Sample=standard Raw Score Difference (ppm) (M13-M14)

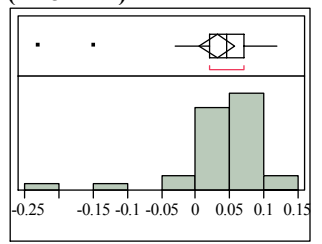

Quantiles

$100.0 \%$ maximum 0.1200

$99.5 \%$

$97.5 \%$

$90.0 \%$

$75.0 \%$ quartile $\quad 0.0700$

$50.0 \%$ median 0.0450

$25.0 \%$ quartile $\quad 0.0200$

$10.0 \% \quad-0.0290$

$\begin{array}{ll}10.0 \% & -0.0290 \\ 2.5 \% & -0.2300\end{array}$

$0.5 \% \quad-0.2300$

$0.0 \%$ minimum -0.2300

Moments

Mean $\quad 0.0316667$

Std Dev $\quad 0.0702745$

Std Err Mean $\quad 0.0128303$

upper 95\% Mean 0.0579076

lower $95 \%$ Mean 0.0054257

$\mathrm{N}$

\section{Exhibit A8j.}

Distributions Anion=Fluoride, Type of Sample=standard Raw Score Difference (ppm) (M13-M14)

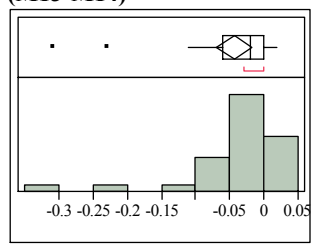

Quantiles

$100.0 \%$ maximum 0.0200

$99.5 \% \quad 0.0200$

$97.5 \% \quad 0.0200$

$90.0 \% \quad 0.0000$

$75.0 \%$ quartile $\quad 0.0000$

$50.0 \%$ median -0.0200

$25.0 \%$ quartile -0.0600

$10.0 \% \quad-0.1090$

$2.5 \% \quad-0.3100$

$0.5 \% \quad-0.3100$

$0.0 \%$ minimum -0.3100

Moments

Mean $\quad-0.043333$

Std Dev $\quad 0.0698932$

Std Err Mean $\quad 0.0127607$

upper $95 \%$ Mean -0.017235

lower 95\% Mean - 0.069432

$\mathrm{N}$
Exhibit A8k.

Distributions Anion=Formate, Type of Sample=process Raw Score Difference (ppm) (M13-M14)

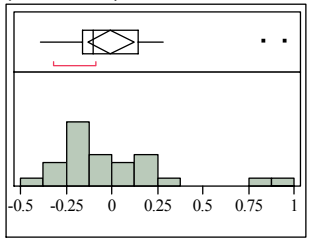

Quantiles

$100.0 \%$ maximum 0.9500

$99.5 \% \quad 0.9500$

$97.5 \% \quad 0.9500$

$90.0 \% \quad 0.4450$

$75.0 \%$ quartile 0.1425

$50.0 \%$ median -0.1000

$25.0 \%$ quartile -0.1625

$10.0 \% \quad-0.3350$

$\begin{array}{ll}10.0 \% & -0.3350 \\ 0.5 \% & -0.3900\end{array}$

$0.5 \% \quad-0.3900$

$0.0 \%$ minimum -0.3900

Moments

Mean $\quad-0.004231$

Std Dev $\quad 0.3173915$

Std Err Mean $\quad 0.0622456$

upper 95\% Mean 0.1239664

lower 95\% Mean - 0.132428

Exhibit A81.

Distributions Anion=Formate, Type of Sample=standard Raw Score Difference (ppm) (M13-M14)

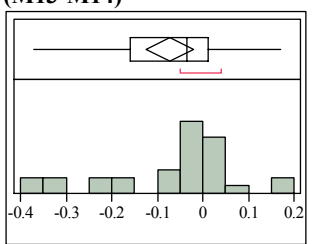

Quantiles

$100.0 \%$ maximum 0.1700

$99.5 \% \quad 0.1700$

$97.5 \% \quad 0.1700$

$90.0 \% \quad 0.0670$

$75.0 \%$ quartile $\quad 0.0125$

$50.0 \%$ median -0.0350

$25.0 \%$ quartile -0.1600

$10.0 \% \quad-0.3480$

$2.5 \% \quad-0.3700$

$0.5 \% \quad-0.3700$

$0.0 \%$ minimum -0.3700

Moments

Mean $\quad-0.072667$

Std Dev $\quad 0.1414929$

Std Err Mean $\quad 0.0258329$

upper 95\% Mean - 0.019832

lower $95 \%$ Mean -0.125501

$\mathrm{N}$
Exhibit A8m.

Distributions Anion=Nitrate, Type of Sample=process

Raw Score Difference (ppm) (M13-M14)

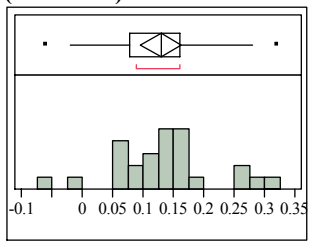

Quantiles

$100.0 \%$ maximum 0.3200

$99.5 \%$

$90.0 \%$

$75.0 \%$ quartile

$50.0 \%$ median

$25.0 \%$ quartile

$10.0 \%$

$2.5 \%$

$0.5 \%$

$0.0 \%$ minimum -0.0600

Moments

Mean $\quad 0.1296154$

Std Dev $\quad 0.0851108$

Std Err Mean $\quad 0.0166916$

upper 95\% Mean 0.1639924

lower $95 \%$ Mean 0.0952384

$\mathrm{N}$

26

Exhibit A8n.

Distributions Anion=Nitrate, Type of Sample=standard

Raw Score Difference (ppm)

(M13-M14)

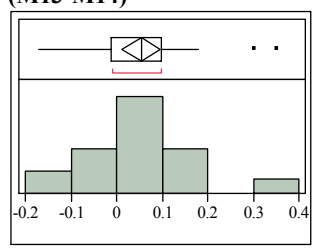

Quantiles

$100.0 \%$ maximum 0.3500

$99.5 \% \quad 0.3500$

$97.5 \% \quad 0.3500$

$90.0 \% \quad 0.1800$

$75.0 \%$ quartile 0.1000

$50.0 \%$ median 0.0550

$25.0 \%$

$10.0 \% \quad-0.1050$

$2.5 \% \quad-0.1700$

$0.5 \% \quad-0.1700$

$0.0 \%$ minimum -0.1700

Moments

Mean $\quad 0.054$

Std Dev $\quad 0.1124829$

Std Err Mean $\quad 0.0205365$

upper $95 \%$ Mean 0.0960018

lower $95 \%$ Mean 0.0119982

$\mathrm{N}$

30
Exhibit A80.

Distributions Anion=Nitrite, Type of Sample=process

Raw Score Difference (ppm) (M13-M14)

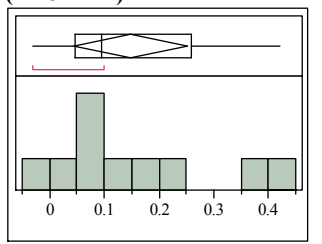

Quantiles

$100.0 \%$ maximum 0.4200

$99.5 \% \quad 0.4200$

$97.5 \% \quad 0.4200$

$90.0 \% \quad 0.4130$

$75.0 \%$ quartile $\quad 0.2600$

$50.0 \%$ median 0.0950

$25.0 \%$ quartile $\quad 0.0475$

$10.0 \% \quad-0.0260$

$\begin{array}{ll}10.0 \% & -0.0260 \\ 0.5 \% & -0.0300\end{array}$

$0.5 \% \quad-0.0300$

$0.0 \%$ minimum -0.0300

Moments

Mean $\quad 0.148$

Std Dev $\quad 0.1455869$

Std Err Mean 0.0460386

upper $95 \%$ Mean 0.2521466

lower 95\% Mean 0.0438534

$\mathrm{N}$

Exhibit A8p.

Distributions Anion=Nitrite, Type of Sample $=$ standard

Raw Score Difference (ppm)

(M13-M14)

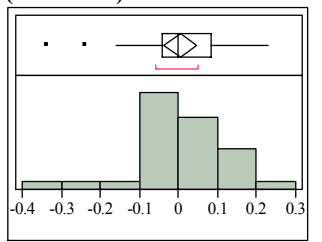

Quantiles

$100.0 \%$ maximum 0.2300

$99.5 \% \quad 0.2300$

$97.5 \%$

$90.0 \% \quad 0.1460$

$75.0 \%$ quartile $\quad 0.0825$

$50.0 \%$ median 0.0000

$25.0 \%$ quartile -0.0425

$10.0 \% \quad-0.1540$

$2.5 \% \quad-0.3400$

$0.5 \% \quad-0.3400$

$0.0 \%$ minimum -0.3400

Moments

Mean $\quad 0.0043333$

Std Dev $\quad 0.1147316$

Std Err Mean $\quad 0.020947$

upper 95\% Mean 0.0471748

lower $95 \%$ Mean -0.038508

$\mathrm{N}$ 
Exhibit A8q.

Distributions Anion=Oxalate, Type of Sample=process

Raw Score Difference (ppm) (M13-M14)

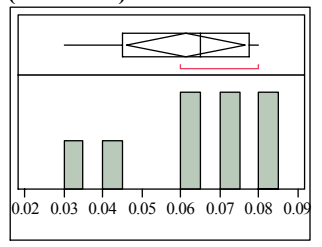

Quantiles

$100.0 \%$ maximum 0.08000

$99.5 \% \quad 0.08000$

$97.5 \% \quad 0.08000$

$90.0 \% \quad 0.08000$

$75.0 \%$ quartile $\quad 0.07750$

$50.0 \%$ median 0.06500

$25.0 \%$ quartile $\quad 0.04500$

$10.0 \% \quad 0.03000$

$\begin{array}{ll}2.5 \% & 0.03000\end{array}$

$0.5 \% \quad 0.03000$

$0.0 \%$ minimum 0.03000

Moments

Mean $\quad 0.06125$

Std Dev $\quad 0.0180772$

Std Err Mean $\quad 0.0063913$

upper $95 \%$ Mean 0.0763629

lower 95\% Mean 0.0461371

$\mathrm{N}$

Exhibit A8r.

Distributions Anion=Oxalate, Type of Sample=standard

Raw Score Difference (ppm)

(M13-M14)

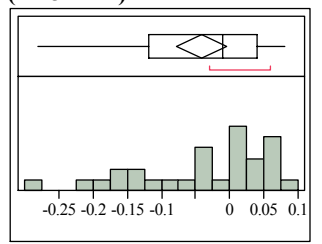

Quantiles

$100.0 \%$ maximum 0.0800

$99.5 \% \quad 0.0800$

$97.5 \% \quad 0.0800$

$90.0 \% \quad 0.0600$

$75.0 \%$ quartile $\quad 0.0400$

$50.0 \%$ median -0.0100

$25.0 \%$ quartile $\quad-0.1175$

$10.0 \% \quad-0.1970$

$2.5 \% \quad-0.2800$

$0.5 \% \quad-0.2800$

$0.0 \%$ minimum -0.2800

Moments

Mean $\quad-0.041333$

Std Dev $\quad 0.0966235$

Std Err Mean $\quad 0.0176409$

upper $95 \%$ Mean -0.005254

lower 95\% Mean - 0.077413

$\mathrm{N}$
Exhibit A8s.

Distributions Anion=Phosphate, Type of Sample $=$ standard Raw Score Difference (ppm) (M13-M14)

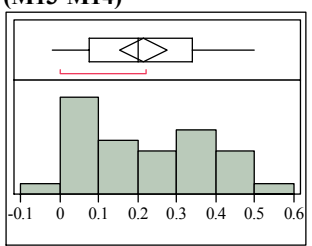

Quantiles

$100.0 \%$ maximum 0.5000

$99.5 \% \quad 0.5000$

$97.5 \% \quad 0.5000$

$90.0 \% \quad 0.4860$

$75.0 \%$ quartile $\quad 0.3400$

$50.0 \%$ median 0.2000

$25.0 \%$ quartile $\quad 0.0775$

$10.0 \% \quad 0.0010$

$\begin{array}{lr}2.5 \% & -0.0200\end{array}$

$0.5 \% \quad-0.0200$

$0.0 \%$ minimum -0.0200

Moments

Mean $\quad 0.2153733$

Std Dev $\quad 0.1615621$

Std Err Mean $\quad 0.0294971$

upper $95 \%$ Mean 0.2757016

lower $95 \%$ Mean 0.1550451

$\mathrm{N}$

Exhibit A8t.

Distributions Anion=Sulfate, Type of Sample=process

Raw Score Difference (ppm)

(M13-M14)

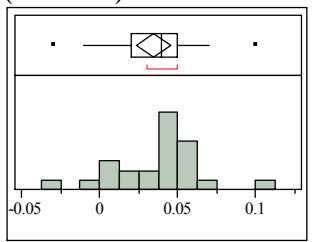

Quantiles

$100.0 \%$ maximum 0.1000

$99.5 \% \quad 0.1000$

$97.5 \% \quad 0.1000$

$90.0 \% \quad 0.0650$

$75.0 \%$ quartile 0.0500

$50.0 \%$ median 0.0400

$25.0 \%$ quartile $\quad 0.0200$

$10.0 \% \quad-0.0050$

$2.5 \% \quad-0.0300$

$0.5 \% \quad-0.0300$

$0.0 \%$ minimum -0.0300

Moments

Mean $\quad 0.0345833$

Std Dev $\quad 0.0266995$

Std Err Mean $\quad 0.00545$

upper $95 \%$ Mean 0.0458575

lower 95\% Mean 0.0233091

$\mathrm{N}$

24
Exhibit A8u.

Distributions Anion=Sulfate, Type of Sample=standard

Raw Score Difference (ppm) (M13-M14)

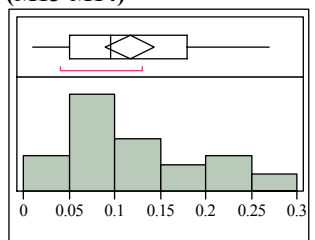

Quantiles

$100.0 \%$ maximum 0.27000

$99.5 \% \quad 0.27000$

$97.5 \% \quad 0.27000$

$90.0 \% \quad 0.21900$

$75.0 \%$ quartile 0.18000

$50.0 \%$ median 0.09500

$25.0 \%$ quartile $\quad 0.05000$

$10.0 \% \quad 0.04000$

$\begin{array}{ll}10.0 \% & 0.04000 \\ 0.5 \% & 0.01000\end{array}$

$0.5 \% \quad 0.01000$

$0.0 \%$ minimum 0.01000

Moments

Mean $\quad 0.1166667$

Std Dev $\quad 0.0709217$

Std Err Mean $\quad 0.0129485$

upper $95 \%$ Mean 0.1431493

lower $95 \%$ Mean 0.0901841

$\mathrm{N}$

30

Exhibit A8v.

Distributions Anion=Chloride, Type of Sample $=$ standard, Tank=SME

Raw Score Difference (ppm) (M13-M14)

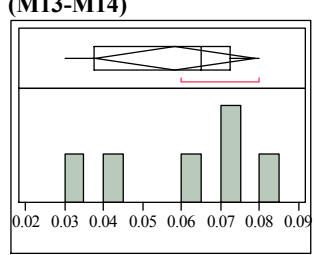

Quantiles

$100.0 \%$ maximum 0.08000

$99.5 \%$

$97.5 \% \quad 0.08000$

$90.0 \%$

$75.0 \%$ quartile

0.08000
0.08000

$50.0 \%$ median $\quad 0.06500$

$25.0 \%$ quartile 0.03750

$10.0 \% \quad 0.03000$

$2.5 \% \quad 0.03000$

$0.5 \% \quad 0.03000$

$0.0 \%$ minimum 0.03000

Moments

Mean

0.0194079

0.0079232

upper $95 \%$ Mean 0.0787007

lower 95\% Mean 0.037966

$\mathrm{N}$
Exhibit A8w.

Distributions Anion=Chloride, Type of Sample $=$ standard, Tank=SRAT Product

Raw Score Difference (ppm) (M13-M14)

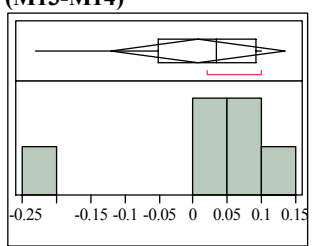

Quantiles

$\begin{array}{llr}100.0 \% & \text { maximum } & 0.1000 \\ 99.5 \% & & 0.1000 \\ 97.5 \% & & 0.1000 \\ 90.0 \% & & 0.1000 \\ 75.0 \% & \text { quartile } & 0.0925 \\ 50.0 \% & \text { median } & 0.0350 \\ 25.0 \% & \text { quartile } & -0.0500 \\ 10.0 \% & & -0.2300 \\ 2.5 \% & & -0.2300 \\ 0.5 \% & -0.2300 \\ 0.0 \% \quad \text { minimum } & -0.2300 \\ \text { Moments } & \\ & \\ \text { Mean } & \\ \text { Std Dev } & 0.0066667 \\ \text { Std Err Mean } & 0.1214359 \\ \text { upper 95\% Mean } & 0.1341058 \\ \text { lower 95\% Mean } & -0.120772 \\ \text { N } & \end{array}$

Exhibit A8x.

Distributions Anion=Chloride,

Type of Sample=standard,

Tank=SRAT Receipt

Raw Score Difference (ppm) (M13-M14)

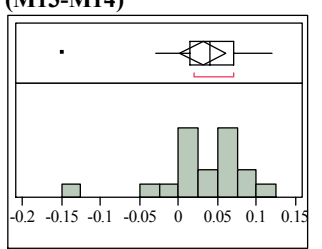

Quantiles

$100.0 \%$ maximum 0.1200

$99.5 \% \quad 0.1200$

$97.5 \% \quad 0.1200$

$90.0 \%$

$75.0 \%$ quartile $\quad 0.0700$

$50.0 \%$ median 0.0400

$25.0 \%$ quartile $\quad 0.0150$

$10.0 \% \quad-0.0420$

$2.5 \% \quad-0.1500$

$0.5 \% \quad-0.1500$

$0.0 \%$ minimum -0.1500

Moments

Mean $\quad 0.031111$

Std Dev $\quad 0.0591995$

Std Err Mean $\quad 0.0139534$

upper 95\% Mean 0.0605503

lower 95\% Mean 0.0016719

$\mathrm{N}$ 
Exhibit A8y.

Distributions Anion=Fluoride, Type of Sample $=$ standard, Tank=SME

Raw Score Difference (ppm) (M13-M14)

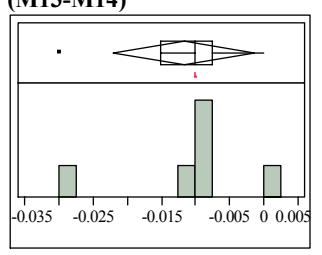

Quantiles

$100.0 \%$ maximum 0.0000

$99.5 \%$

$97.5 \%$

$90.0 \%$

artile $\quad-0.0075$

$50.0 \%$ median -0.0100

$25.0 \%$ quartile $\quad-0.0150$

$10.0 \% \quad-0.0300$

$2.5 \% \quad-0.0300$

$0.5 \% \quad-0.0300$

$0.0 \%$ minimum -0.0300

Moments

Mean $\quad-0.011667$

Std Dev $\quad 0.0098319$

Std Err Mean $\quad 0.0040139$

upper $95 \%$ Mean $\quad-0.001349$

lower 95\% Mean - 0.021985

$\mathrm{N}$

Exhibit A8z.

Distributions Anion=Fluoride, Type of Sample=standard,

Tank=SRAT Product

Raw Score Difference (ppm) (M13-M14)

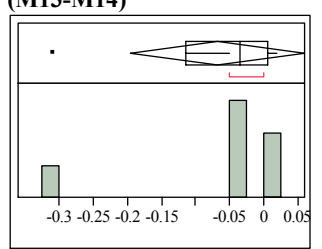

Quantiles

$100.0 \%$ maximum 0.0200

$99.5 \% \quad 0.0200$

$97.5 \% \quad 0.0200$

$90.0 \% \quad 0.0200$

$75.0 \%$ quartile $\quad 0.0050$

$50.0 \%$ median -0.0350

$25.0 \%$ quartile $\quad-0.1150$

$10.0 \% \quad-0.3100$

$2.5 \% \quad-0.3100$

$0.5 \% \quad-0.3100$

$0.0 \%$ minimum -0.3100

Moments

Mean $\quad-0.068333$

Std Dev $\quad 0.1212298$

Std Err Mean $\quad 0.0494919$

upper $95 \%$ Mean 0.0588895

lower $95 \%$ Mean - 0.195556
Exhibit A8aa.

Distributions Anion=Fluoride, Type of Sample=standard, Tank=SRAT Receipt

Raw Score Difference (ppm) (M13-M14)

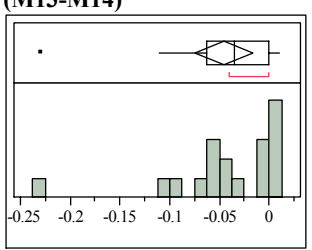

Quantiles

$\begin{array}{lrr}100.0 \% & \text { maximum } & 0.0100 \\ 99.5 \% & & 0.0100 \\ 97.5 \% & & 0.0100 \\ 90.0 \% & & 0.0010 \\ 75.0 \% & \text { quartile } & 0.0000 \\ 50.0 \% & \text { median } & -0.0350 \\ 25.0 \% & \text { quartile } & -0.0625 \\ 10.0 \% & & -0.1220 \\ 2.5 \% & -0.2300 \\ 0.5 \% & -0.2300 \\ 0.0 \% \quad \text { minimum } & -0.2300 \\ \text { Moments } & \\ & \\ \text { Mean } & -0.045556 \\ \text { Std Dev } & 0.0583319 \\ \text { Std Err Mean } & 0.013749 \\ \text { upper 95\% Mean } & -0.016548 \\ \text { lower 95\% Mean } & -0.074563 \\ \text { N } & \end{array}$

Exhibit A8ab.

Distributions Anion=Formate, Type of Sample=process,

Tank=SME

Raw Score Difference (ppm) (M13-M14)

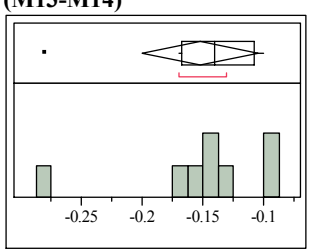

Quantiles

$100.0 \%$ maximum -0.1000

$\begin{array}{ll}99.5 \% & -0.1000 \\ 97.5 \% & -0.1000\end{array}$

$90.0 \% \quad-0.1000$

$75.0 \%$ quartile $\quad-0.1075$

$50.0 \%$ median -0.1400

$25.0 \%$ quartile $\quad-0.1675$

$10.0 \% \quad-0.2800$

$2.5 \% \quad-0.2800$

$0.5 \% \quad-0.2800$

$0.0 \%$ minimum -0.2800

Moments

Mean $\quad-0.1525$

Std Dev $\quad 0.0572588$

Std Err Mean $\quad 0.020244$

upper $95 \%$ Mean $\quad-0.10463$

lower 95\% Mean $\quad-0.20037$
Exhibit A8ac.

Distributions Anion=Formate,

Type of Sample=process,

Tank=SRAT Product

Raw Score Difference (ppm)

(M13-M14)

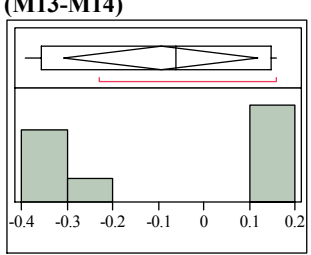

Quantiles

$\begin{array}{lrr}100.0 \% & \text { maximum } & 0.1600 \\ 99.5 \% & & 0.1600 \\ 97.5 \% & & 0.1600 \\ 90.0 \% & & 0.1600 \\ 75.0 \% & \text { quartile } & 0.1475 \\ 50.0 \% & \text { median } & -0.0600 \\ 25.0 \% & \text { quartile } & -0.3575 \\ 10.0 \% & & -0.3900 \\ 2.5 \% & & -0.3900 \\ 0.5 \% & -0.3900 \\ 0.0 \% \quad \text { minimum } & -0.3900 \\ \text { Moments } & \\ & \\ \text { Mean } & \\ \text { Std Dev } & -0.09375 \\ \text { Std Err Mean r } & 0.254611 \\ \text { upper 95\% Mean } & 0.1191102 \\ \text { lower 95\% Mean } & -0.30661 \\ \text { N } & 8\end{array}$

Exhibit A8ad.

Distributions Anion=Formate, Type of Sample=process,

Tank=SRAT Receipt

Raw Score Difference (ppm) (M13-M14)

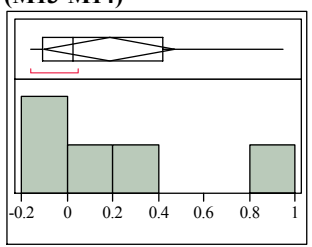

Quantiles

$100.0 \%$ maximum 0.9500

$99.5 \% \quad 0.9500$

$97.5 \% \quad 0.9500$

$90.0 \% \quad 0.9380$

$75.0 \%$ quartile $\quad 0.4175$

$50.0 \%$ median 0.0250

$25.0 \%$ quartile $\quad-0.1075$

$10.0 \% \quad-0.1570$

$2.5 \% \quad-0.1600$

$0.5 \% \quad-0.1600$

$0.0 \%$ minimum -0.1600

Moments

Mean

0.3999778

Std Err Mean $\quad 0.1264841$

upper $95 \%$ Mean 0.4721269

lower $95 \%$ Mean - 0.100127

$\mathrm{N}$
Exhibit A8ae.

Distributions Anion=Formate, Type of Sample $=$ standard, Tank=SME

Raw Score Difference (ppm) (M13-M14)

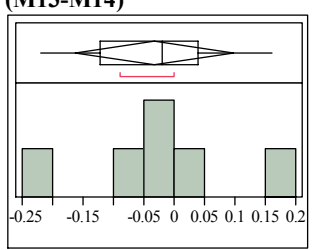

Quantiles

$100.0 \%$ maximum 0.1600

$99.5 \% \quad 0.1600$

$97.5 \% \quad 0.1600$

$90.0 \% \quad 0.1600$

$75.0 \%$ quartile $\quad 0.0400$

$50.0 \%$ median -0.0200

$25.0 \%$ quartile -0.1225

$10.0 \% \quad-0.2200$

$2.5 \% \quad-0.2200$

$0.5 \% \quad-0.2200$

$0.0 \%$ minimum -0.2200

Moments

Mean $\quad-0.031667$

$\begin{array}{ll}\text { Std Dev } & 0.1241639 \\ \text { Std Err Mean } & 0.0506897\end{array}$

upper 95\% Mean 0.0986353

lower 95\% Mean -0.161969

Exhibit A8af.

Distributions Anion=Formate,

Type of Sample=standard,

Tank=SRAT Product

Raw Score Difference (ppm) (M13-M14)

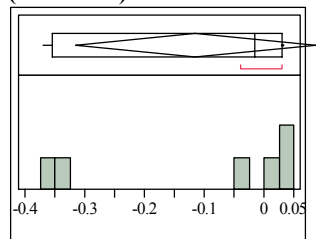

Quantiles

$100.0 \%$ maximum 0.0300

$99.5 \% \quad 0.0300$

$\begin{array}{ll}97.5 \% & 0.0300\end{array}$

$90.0 \% \quad 0.0300$

$75.0 \%$ quartile $\quad 0.0300$

$50.0 \%$ median -0.0150

$25.0 \%$ quartile $\quad-0.3550$

$10.0 \% \quad-0.3700$

$2.5 \% \quad-0.3700$

$0.5 \% \quad-0.3700$

$0.0 \%$ minimum -0.3700

Moments

Mean $\quad-0.115$

Std Dev $\quad 0.1915985$

Std Err Mean $\quad 0.0782198$

upper 95\% Mean 0.0860703

lower $95 \%$ Mean $\quad-0.31607$

$\mathrm{N}$ 
Exhibit A8ag.

Distributions Anion=Formate,

Type of Sample=standard,

Tank=SRAT Receipt

Raw Score Difference (ppm)

(M13-M14)

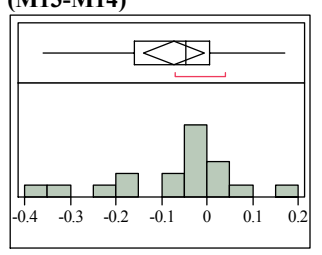

Quantiles

$100.0 \%$ maximum 0.1700

$99.5 \%$

$97.5 \%$

$90.0 \%$

$25.0 \%$ quartile $\quad-0.1600$

$10.0 \% \quad-0.3330$

$2.5 \%--0.3600$

$0.5 \% \quad-0.3600$

$0.0 \% \quad$ minimum -0.3600

Moments

Mean $\quad-0.072222$

Std Dev $\quad 0.1326453$

Std Err Mean 0.0312648

upper $95 \%$ Mean -0.006259

lower 95\% Mean -0.138185

$\mathrm{N}$

Exhibit A8ah.

Distributions Anion=Nitrate, Type of Sample=process, Tank $=\mathrm{SME}$

Raw Score Difference (ppm) (M13-M14)

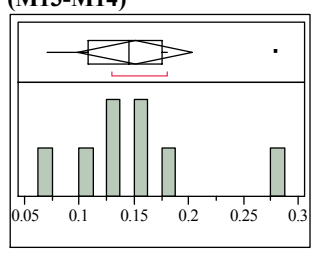

Quantiles

$100.0 \%$ maximum 0.28000

$99.5 \% \quad 0.28000$

$97.5 \% \quad 0.28000$

$90.0 \% \quad 0.28000$

$75.0 \%$ quartile $\quad 0.17500$

$50.0 \%$ median 0.14500

$25.0 \%$ quartile 0.10750

$10.0 \% \quad 0.07000$

$2.5 \%-0.07000$

$0.5 \% \quad 0.07000$

$0.0 \%$ minimum 0.07000

Moments

Mean $\quad 0.15125$

Std Dev $\quad 0.0628916$

Std Err Mean $\quad 0.0222355$

upper $95 \%$ Mean 0.2038287

lower $95 \%$ Mean 0.0986713
Exhibit A8ai.

Distributions Anion=Nitrate, Type of Sample=process, Tank=SRAT Product

Raw Score Difference (ppm) (M13-M14)

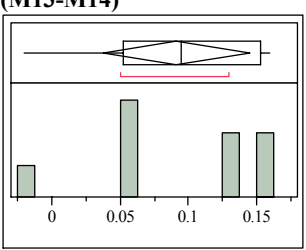

Quantiles

\begin{tabular}{|c|c|}
\hline & \\
\hline $100.0 \%$ maximum & n 0.1600 \\
\hline $99.5 \%$ & 0.1600 \\
\hline $97.5 \%$ & 0.1600 \\
\hline $90.0 \%$ & 0.1600 \\
\hline $75.0 \%$ quartile & 0.1525 \\
\hline $50.0 \%$ median & 0.0950 \\
\hline $25.0 \%$ quartile & 0.0525 \\
\hline $10.0 \%$ & -0.0200 \\
\hline $2.5 \%$ & -0.0200 \\
\hline $0.5 \%$ & -0.0200 \\
\hline minimum & -0.0200 \\
\hline Moments & \\
\hline Mean & 0.09125 \\
\hline Std Dev & 0.0637938 \\
\hline Std Err Mean & 0.0225545 \\
\hline upper $95 \%$ Mean & 0.1445829 \\
\hline lower $95 \%$ Mean & 0.0379171 \\
\hline
\end{tabular}

Exhibit A8aj.

Distributions Anion=Nitrate, Type of Sample=process, Tank $=$ SRAT Receipt

Raw Score Difference (ppm) (M13-M14)

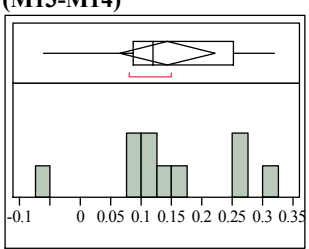

Quantiles

$\begin{array}{lll}100.0 \% & \text { maximum } & 0.3200 \\ 99.5 \% & & 0.3200 \\ 97.5 \% & & 0.3200 \\ 90.0 \% & & 0.3140 \\ 75.0 \% & \text { quartile } & 0.2525 \\ 50.0 \% & \text { median } & 0.1200 \\ 25.0 \% & \text { quartile } & 0.0875 \\ 10.0 \% & & -0.0460 \\ 2.5 \% & & -0.0600 \\ 0.5 \% & & -0.0600 \\ 0.0 \% & \text { minimum } & -0.0600\end{array}$

Moments

Mean $\quad 0.143$

Std Dev $\quad 0.1093465$

Std Err Mean $\quad 0.0345784$

upper $95 \%$ Mean 0.2212218

lower $95 \%$ Mean 0.0647782
Exhibit A8ak.

Distributions Anion=Nitrate, Type of Sample=standard, Tank=SME Raw Score Difference (ppm) (M13-M14)

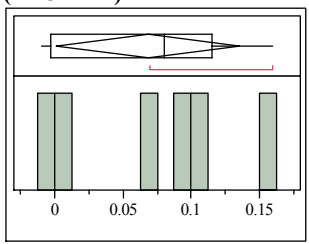

Quantiles

$\begin{array}{lrr}100.0 \% & \text { maximum } & 0.1600 \\ 99.5 \% & & 0.1600 \\ 97.5 \% & & 0.1600 \\ 90.0 \% & & 0.1600 \\ 75.0 \% & \text { quartile } & 0.1150 \\ 50.0 \% & \text { median } & 0.0800 \\ 25.0 \% & \text { quartile } & -0.0025 \\ 10.0 \% & & -0.0100 \\ 2.5 \% & & -0.0100 \\ 0.5 \% & -0.0100 \\ 0.0 \% \quad \text { minimum } & -0.0100 \\ \text { Moments } & \\ & \\ \text { Mean } & \\ \text { Std Dev } & 0.0683333 \\ \text { Std Err Mean } & 0.0643169 \\ \text { upper 95\% Mean } & 0.0262573 \\ \text { lower 95\% Mean } & 0.0008369 \\ \text { N } & 6\end{array}$

Exhibit A8al.

Distributions Anion=Nitrate, Type of Sample=standard, Tank $=$ SRAT Product

Raw Score Difference (ppm) (M13-M14)

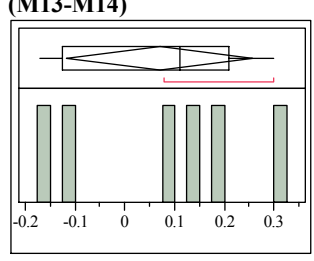

Quantiles

$\begin{array}{lll}100.0 \% & \text { maximum } & 0.3000 \\ 99.5 \% & & 0.3000 \\ 97.5 \% & & 0.3000 \\ 90.0 \% & & 0.3000 \\ 75.0 \% & \text { quartile } & 0.2100 \\ 50.0 \% & \text { median } & 0.1100 \\ 25.0 \% & \text { quartile } & -0.1250 \\ 10.0 \% & & -0.1700 \\ 2.5 \% & & -0.1700 \\ 0.5 \% & & -0.1700 \\ 0.0 \% & \text { minimum } & -0.1700\end{array}$

Moments

Mean 0.07 Std Dev $\quad 0.1788854$ Std Err Mean $\quad 0.0730297$ upper $95 \%$ Mean 0.2577288 lower 95\% Mean - 0.117729

$\mathrm{N}$
Exhibit A8am.

Distributions Anion=Nitrate, Type of Sample=standard, Tank=SRAT Receipt

Raw Score Difference (ppm) (M13-M14)

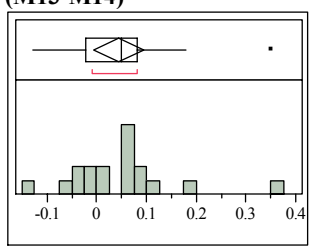

Quantiles

$\begin{array}{lrr}100.0 \% & \text { maximum } & 0.3500 \\ 99.5 \% & & 0.3500 \\ 97.5 \% & & 0.3500 \\ 90.0 \% & & 0.1970 \\ 75.0 \% & \text { quartile } & 0.0800 \\ 50.0 \% & \text { median } & 0.0500 \\ 25.0 \% & \text { quartile } & -0.0225 \\ 10.0 \% & & -0.0670 \\ 2.5 \% & & -0.1300 \\ 0.5 \% & -0.1300 \\ 0.0 \% \quad \text { minimum } & -0.1300 \\ \text { Moments } & \\ & \\ \text { Mean } & \\ \text { Std Dev } & 0.0438889 \\ \text { Std Err Mean } & 0.1033634 \\ \text { upper 95\% Mean } & 0.024363 \\ \text { lower 95\% Mean } & -0.0052903 \\ \text { N } & \end{array}$

Exhibit A8an.

Distributions Anion=Nitrite, Type of Sample $=$ process, Tank $=$ SRAT

Receipt

Raw Score Difference (ppm) (M13-M14)

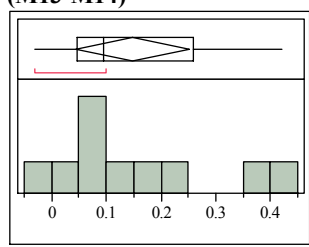

Quantiles

$100.0 \%$ maximum 0.4200

$99.5 \% \quad 0.4200$

$\begin{array}{ll}97.5 \% & 0.4200\end{array}$

$90.0 \%$

$75.0 \%$ quartile $\quad 0.2600$

$50.0 \%$ median 0.0950

$25.0 \%$ quartile $\quad 0.0475$

$10.0 \% \quad-0.0260$

$2.5 \% \quad-0.0300$

$0.5 \% \quad-0.0300$

$0.0 \%$ minimum -0.0300

Moments

Mean $\quad 0.148$

Std Dev $\quad 0.1455869$

Std Err Mean 0.0460386 upper 95\% Mean 0.2521466 lower 95\% Mean 0.0438534 
Exhibit A8ao.

Distributions Anion=Nitrite, Type of Sample=standard, Tank=SME Raw Score Difference (ppm) (M13-M14)

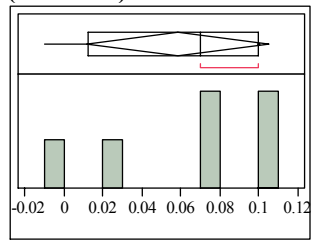

Quantiles

$100.0 \%$ maximum 0.1000

$99.5 \%$

$97.5 \%$

$90.0 \%$

$75.0 \%$ quartile $\quad 0.1000$

$50.0 \%$ median 0.0700

$25.0 \%$ quartile

$10.0 \% \quad-0.0100$

$\begin{array}{ll}10.0 \% & -0.0100 \\ 0.5 \% & -0.0100\end{array}$

$0.5 \% \quad-0.0100$

$0.0 \%$ minimum -0.0100

Moments

Mean $\quad 0.0583333$

Std Dev $\quad 0.0444597$

Std Err Mean $\quad 0.0181506$

upper 95\% Mean 0.1049909

lower $95 \%$ Mean 0.0116757

$\mathrm{N}$

\section{Exhibit A8ap.}

Distributions Anion=Nitrite, Type of Sample $=$ standard, Tank=SRAT Product

Raw Score Difference (ppm) (M13-M14)

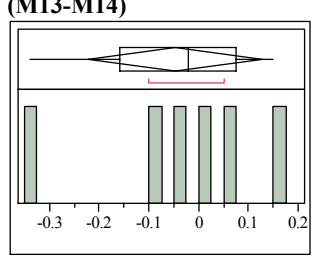

Quantiles

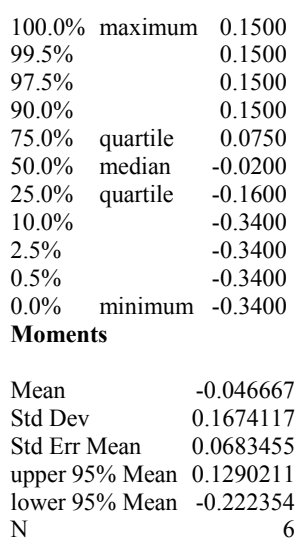

Exhibit A8aq.

Distributions Anion=Nitrite, Type of Sample=standard, Tank=SRAT Receipt

Raw Score Difference (ppm) (M13-M14)

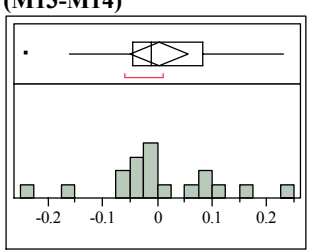

Quantiles

$\begin{array}{lrr}100.0 \% & \text { maximum } & 0.2300 \\ 99.5 \% & & 0.2300 \\ 97.5 \% & & 0.2300 \\ 90.0 \% & & 0.1580 \\ 75.0 \% & \text { quartile } & 0.0825 \\ 50.0 \% & \text { median } & -0.0100 \\ 25.0 \% & \text { quartile } & -0.0450 \\ 10.0 \% & & -0.1680 \\ 2.5 \% & & -0.2400 \\ 0.5 \% & -0.2400 \\ 0.0 \% \text { minimum } & -0.2400 \\ \text { Moments } & \\ & \\ \text { Mean } & 0.0033333 \\ \text { Std Dev } & 0.1080849 \\ \text { Std Err Mean } & 0.0254759 \\ \text { upper 95\% Mean } & 0.0570827 \\ \text { lower 95\% Mean } & -0.050416 \\ \text { N } & 18\end{array}$

Exhibit A8ar.

Distributions Anion=Oxalate, Type of Sample=process, Tank $=$ SRAT

Receipt

Raw Score Difference (ppm) (M13-M14)

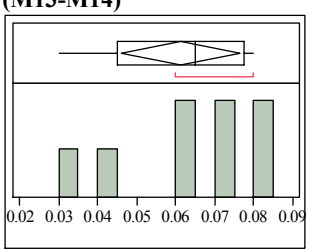

Quantiles

$100.0 \%$ maximum 0.08000

$99.5 \% \quad 0.08000$

$97.5 \% \quad 0.08000$

$90.0 \% \quad 0.08000$

$75.0 \%$ quartile $\quad 0.07750$

$50.0 \%$ median 0.06500

$25.0 \%$ quartile $\quad 0.04500$

$10.0 \% \quad 0.03000$

$\begin{array}{ll}2.5 \% & 0.03000\end{array}$

$0.5 \% \quad 0.03000$

$0.0 \%$ minimum 0.03000

Moments

Mean $\quad 0.06125$

Std Dev $\quad 0.0180772$

Std Err Mean $\quad 0.0063913$

upper $95 \%$ Mean 0.0763629

lower 95\% Mean 0.0461371
Exhibit A8as.

Distributions Anion=Oxalate, Type of Sample=standard, Tank $=$ SME Raw Score Difference (ppm) (M13-M14)

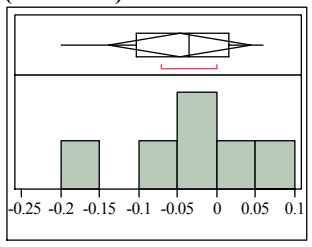

Quantiles

$100.0 \%$ maximum 0.0600

$99.5 \% \quad 0.0600$

$97.5 \% \quad 0.0600$

$90.0 \% \quad 0.0600$

$75.0 \%$ quartile $\quad 0.0150$

$50.0 \%$ median -0.0350

$25.0 \%$ quartile $\quad-0.1025$

$10.0 \% \quad-0.2000$

$\begin{array}{ll}10.0 \% & -0.2000\end{array}$

$0.5 \% \quad-0.2000$

$0.0 \%$ minimum -0.2000

Moments

Mean $\quad-0.046667$

Std Dev $\quad 0.0871015$

Std Err Mean $\quad 0.035559$

upper 95\% Mean 0.0447407

lower $95 \%$ Mean -0.138074

$\mathrm{N}$

Exhibit A8at.

Distributions Anion=Oxalate, Type of Sample $=$ standard, Tank $=$ SRAT Product

Raw Score Difference (ppm) (M13-M14)

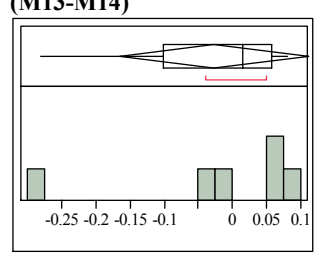

Quantiles

$100.0 \%$ maximum 0.0800

$99.5 \%$

$97.5 \% \quad 0.0800$

$75.0 \%$ quartile

$50.0 \%$ median

$25.0 \%$ quartile

$10.0 \%$

$2.5 \%$

$0.5 \%$

Moments

Mean

Std Dev $\quad 0.1323128$

Std Err Mean $\quad 0.0540165$

upper 95\% Mean 0.1121871

lower $95 \%$ Mean $\quad-0.16552$

$\mathrm{N}$
Exhibit A8au.

Distributions Anion=Oxalate, Type of Sample=standard, Tank=SRAT Receipt

Raw Score Difference (ppm) (M13-M14)

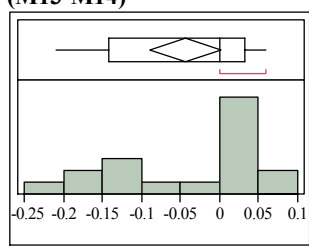

Quantiles

$100.0 \%$ maximum 0.0600

$99.5 \% \quad 0.0600$

$97.5 \% \quad 0.0600$

$90.0 \% \quad 0.0600$

$75.0 \%$ quartile $\quad 0.0325$

$50.0 \%$ median 0.0000

$25.0 \%$ quartile -0.1425

$10.0 \% \quad-0.1740$

$2.5 \% \quad-0.2100$

$0.5 \% \quad-0.2100$

$0.0 \% \quad$ minimum -0.2100

Moments

Mean $\quad-0.044444$

Std Dev $\quad 0.0919221$

Std Err Mean $\quad 0.0216662$

upper $95 \%$ Mean 0.0012673

lower $95 \%$ Mean -0.090156

18

Exhibit A8av.

Distributions Anion=Phosphate,

Type of Sample $=$ standard,

Tank=SME

Raw Score Difference (ppm) (M13-M14)

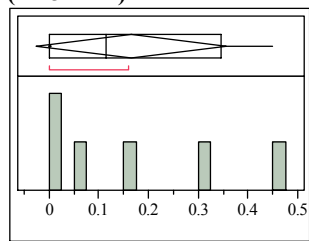

Quantiles

$100.0 \%$ maximum 0.45000

$99.5 \% \quad 0.45000$

$97.5 \% \quad 0.45000$

$90.0 \% \quad 0.45000$

$75.0 \%$ quartile $\quad 0.34500$

$50.0 \%$ median 0.11500

$25.0 \%$ quartile $\quad 0.00000$

$10.0 \% \quad 0.00000$

$2.5 \% \quad 0.00000$

$0.5 \% \quad 0.00000$

$0.0 \%$ minimum 0.00000

Moments

Mean $\quad 0.165$

Std Dev $\quad 0.1820714$

Std Err Mean 0.0743303

upper 95\% Mean 0.3560722

lower 95\% Mean - 0.026072 
Exhibit A8aw.

Distributions Anion=Phosphate, Type of Sample=standard, Tank=SRAT Product

Raw Score Difference (ppm) (M13-M14)

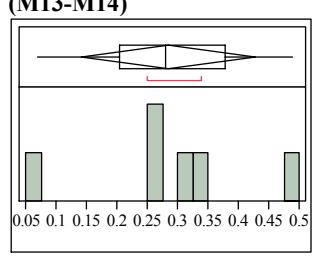

Quantiles

$100.0 \%$ maximum 0.4900

$97.5 \%$

$90.0 \%$

artile $\quad 0.37750$

$25.0 \%$ quartile $\quad 0.20500$

$10.0 \% \quad 0.07000$

$2.5 \% \quad 0.07000$

$0.5 \% \quad 0.07000$

$0.0 \%$ minimum 0.07000

Moments

Mean $\quad 0.285$

Std Dev $\quad 0.1373681$

upper 95\% Mean 0.429159

lower 95\% Mean 0.140841

Exhibit A8ax.

Distributions Anion=Phosphate,

Type of Sample=standard,

Tank=SRAT Receipt

Raw Score Difference (ppm) (M13-M14)

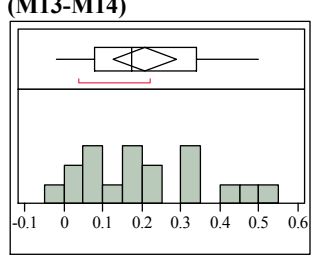

Quantiles

$100.0 \%$ maximum 0.5000

$97.5 \%$

$90.0 \%$

0.4910

$75.0 \%$ quartile $\quad 0.3400$

$50.0 \%$ median 0.1750

$25.0 \%$ quartile $\quad 0.0800$

$10.0 \% \quad 0.0070$

$2.5 \% \quad-0.0200$

$0.5 \% \quad-0.0200$

$0.0 \%$ minimum -0.0200

Moments

Mean $\quad 0.2089556$

Std Dev $\quad 0.1630253$

Std Err Mean $\quad 0.0384254$

upper 95\% Mean 0.2900261

lower 95\% Mean $\quad 0.127885$
Exhibit A8ay.

Distributions Anion=Sulfate, Type of Sample=process, Tank $=$ SME Raw Score Difference (ppm) (M13-M14)

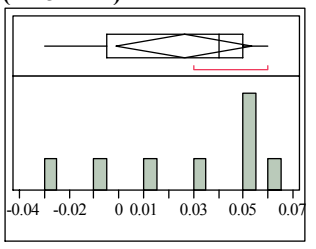

Quantiles

$100.0 \%$ maximum 0.0600

$75.0 \%$ quartile

$50.0 \%$ median

$10.0 \%$

$0.0 \%$ minimum -0.0300

Moments

Mean $\quad 0.02625$

Std Dev $\quad 0.0329231$

Std Err Mean $\quad 0.0116401$

upper 95\% Mean 0.0537744

lower $95 \%$ Mean -0.001274

$\mathrm{N}$

Exhibit A8az.

Distributions Anion=Sulfate, Type of Sample=process, Tank $=$ SRAT Product

Raw Score Difference (ppm) (M13-M14)

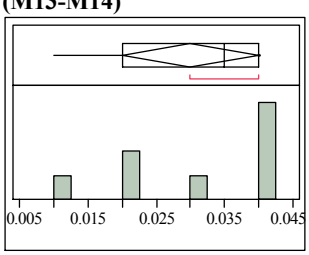

Quantiles

$100.0 \%$ maximum 0.04000

$9.5 \% \quad 0.04000$

$\begin{array}{ll}97.5 \% & 0.04000\end{array}$

$75.0 \%$ quartile $\quad 0.04000$

$50.0 \%$ median 0.03500

$25.0 \%$ quartile 0.02000

$10.0 \% \quad 0.01000$

$2.5 \% \quad 0.01000$

$0.5 \% \quad 0.01000$

$0.0 \%$ minimum 0.01000

Moments

Mean

0.0119523

0.0042258

upper 95\% Mean 0.0399924

lower $95 \%$ Mean 0.0200076

$\mathrm{N}$
Exhibit A8ba.

Distributions Anion=Sulfate, Type of Sample=process, Tank $=$ SRAT Receipt

Raw Score Difference (ppm) (M13-M14)

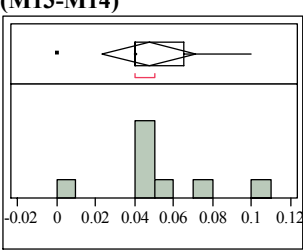

Quantiles

\begin{tabular}{|c|c|}
\hline $100.0 \%$ maximum & 0.10000 \\
\hline $99.5 \%$ & 0.10000 \\
\hline $97.5 \%$ & 0.10000 \\
\hline $90.0 \%$ & 0.10000 \\
\hline $75.0 \%$ quartile & 0.06500 \\
\hline $50.0 \%$ median & 0.04000 \\
\hline $25.0 \%$ quartile & 0.04000 \\
\hline $10.0 \%$ & 0.00000 \\
\hline $2.5 \%$ & 0.00000 \\
\hline $0.5 \%$ & 0.00000 \\
\hline minimum & 0.00000 \\
\hline \multicolumn{2}{|l|}{ Moments } \\
\hline Mean & 0.0475 \\
\hline Std Dev & 0.0286606 \\
\hline Std Err Mean & 0.010133 \\
\hline upper $95 \%$ Mean & 0.0714608 \\
\hline lower $95 \%$ Mean & 0.0235392 \\
\hline
\end{tabular}

Exhibit A8bb.

Distributions Anion=Sulfate, Type of Sample $=$ standard, Tank $=$ SME

Raw Score Difference (ppm) (M13-M14)

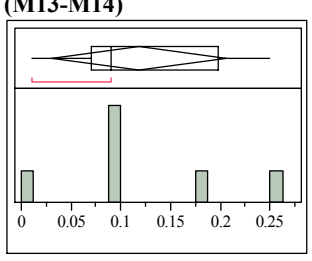

Quantiles

$100.0 \%$ maximum 0.25000

$99.5 \% \quad 0.25000$

$97.5 \%-0.25000$

$90.0 \% \quad 0.25000$

$75.0 \%$ quartile $\quad 0.1975$

$50.0 \%$ median 0.09000

$25.0 \%$ quartile 0.07000

$10.0 \% \quad 0.01000$

$2.5 \% \quad 0.01000$

$0.5 \% \quad 0.01000$

$0.0 \%$ minimum 0.01000

Moments

Mean $\quad 0.1183333$

Std Dev $\quad 0.084004$

Std Err Mean $\quad 0.0342945$

upper 95\% Mean 0.2064901

lower 95\% Mean 0.0301766

$\mathrm{N}$
Exhibit A8bc.

Distributions Anion=Sulfate, Type of Sample=standard, Tank=SRAT Product

Raw Score Difference (ppm)

(M13-M14)

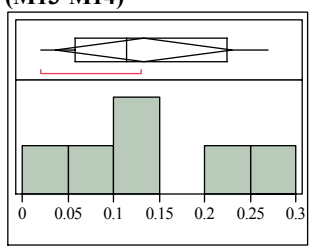

Quantiles

$100.0 \%$ maximum 0.27000

$99.5 \% \quad 0.27000$

$97.5 \% \quad 0.27000$

$90.0 \% \quad 0.27000$

$75.0 \%$ quartile $\quad 0.22500$

$50.0 \%$ median

$25.0 \%$ quartile 0.05750

$10.0 \% \quad 0.02000$

$2.5 \% \quad 0.02000$

$0.5 \% \quad 0.02000$

$0.0 \%$ minimum 0.02000

Moments

Mean $\quad 0.1333333$

$\begin{array}{ll}\text { Std Dev } & 0.0922316 \\ \text { Std Err Mean } & 0.0376534\end{array}$

upper 95\% Mean 0.2301245

lower 95\% Mean 0.0365422

Exhibit A8bd.

Distributions Anion=Sulfate, Type of Sample=standard, Tank=SRAT

Receipt

Raw Score Difference (ppm) (M13-M14)

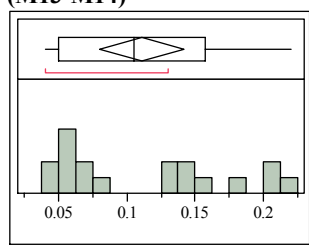

Quantiles

$100.0 \%$ maximum 0.22000

$99.5 \% \quad 0.22000$

$\begin{array}{ll}97.5 \% & 0.22000\end{array}$

$90.0 \% \quad 0.20200$

$75.0 \%$ quartile $\quad 0.15750$

$50.0 \%$ median 0.10500

$25.0 \%$ quartile $\quad 0.05000$

$10.0 \% \quad 0.04000$

$2.5 \% \quad 0.04000$

$0.5 \% \quad 0.04000$

$0.0 \%$ minimum 0.04000

Moments

Mean $\quad 0.1105556$

Std Dev $\quad 0.0621641$

Std Err Mean $\quad 0.0146522$

upper 95\% Mean 0.1414691

lower 95\% Mean 0.0796421 
Exhibit A8be.

Distributions Anion=Chloride, Type of Sample=standard, Bottle ID $=16 \mathrm{ppm}$

Raw Score Difference (ppm) (M13-M14)

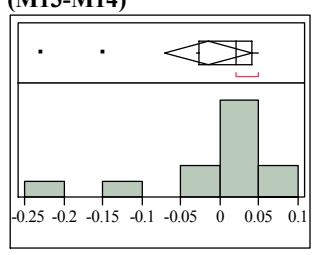

Quantiles

$100.0 \%$ maximum 0.0500

$99.5 \%-0.0500$

$97.5 \%-0.0500$

$90.0 \% \quad 0.0500$

$75.0 \%$ quartile $\quad 0.0400$

$50.0 \%$ median

$25.0 \%$ quartile -0.0275

$10.0 \% \quad-0.2060$

$2.5 \%-0.2300$

$0.5 \% \quad-0.2300$

$0.0 \%$ minimum -0.2300

Moments

Mean $\quad-0.015$

Std Dev $\quad 0.0873343$

Std Err Mean $\quad 0.0252112$

upper 95\% Mean 0.0404895

lower 95\% Mean $\quad-0.07049$

$\mathrm{N}$

12

Exhibit A8bf.

Distributions Anion=Chloride,

Type of Sample $=$ standard, Bottle

ID=2ppm

Raw Score Difference (ppm)

(M13-M14)

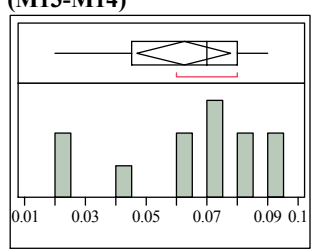

Quantiles

$100.0 \%$ maximum 0.09000

$99.5 \% \quad 0.09000$

$\begin{array}{ll}97.5 \% & 0.09000\end{array}$

$90.0 \% \quad 0.09000$

$75.0 \%$ quartile $\quad 0.08000$

$50.0 \%$ median 0.07000

$25.0 \%$ quartile 0.04500

$10.0 \% \quad 0.02000$

$2.5 \% \quad 0.02000$

$0.5 \% \quad 0.02000$

$0.0 \%$ minimum 0.02000

Moments

Mean $\quad 0.0625$

Std Dev $\quad 0.024168$

Std Err Mean $\quad 0.0069767$

upper $95 \%$ Mean 0.0778556

lower $95 \%$ Mean 0.0471444

$\mathrm{N}$
Exhibit A8bg.

Distributions Anion=Fluoride,

Type of Sample=standard, Bottle

ID=16ppm

Raw Score Difference (ppm) (M13-M14)

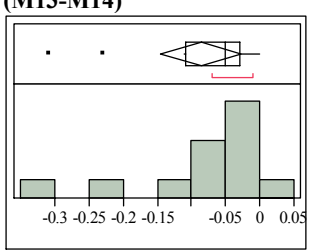

Quantiles

$\begin{array}{lrr}100.0 \% & \text { maximum } & 0.0000 \\ 99.5 \% & & 0.0000 \\ 97.5 \% & & 0.0000 \\ 90.0 \% & & -0.0030 \\ 75.0 \% & \text { quartile } & -0.0300 \\ 50.0 \% & \text { median } & -0.0500 \\ 25.0 \% & \text { quartile } & -0.1075 \\ 10.0 \% & & -0.2860 \\ 2.5 \% & -0.3100 \\ 0.5 \% & -0.3100 \\ 0.0 \% \quad \text { minimum } & -0.3100 \\ \text { Moments } & \\ & \\ \text { Mean } & -0.085833 \\ \text { Std Dev } & 0.0935617 \\ \text { Std Err Mean } & 0.0270089 \\ \text { upper 95\% Mean } & -0.026387 \\ \text { lower 95\% Mean } & -0.14528 \\ \text { N } & \end{array}$

$\mathrm{N}$

Exhibit A8bh.

Distributions Anion=Fluoride,

Type of Sample=standard, Bottle

ID=2ppm

Raw Score Difference (ppm)

(M13-M14)

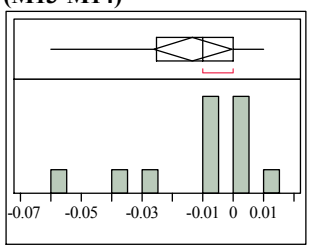

Quantiles

$100.0 \%$ maximum 0.0100

$99.5 \% \quad 0.0100$

$\begin{array}{ll}97.5 \% & 0.0100\end{array}$

$90.0 \% \quad 0.0070$

$75.0 \%$ quartile $\quad 0.0000$

$50.0 \%$ median -0.0100

$25.0 \%$ quartile $\quad-0.0250$

$10.0 \% \quad-0.0540$

$2.5 \% \quad-0.0600$

$0.5 \% \quad-0.0600$

$0.0 \%$ minimum -0.0600

Moments

Mean $\quad-0.013333$

Std Dev $\quad 0.0201509$

Std Err Mean $\quad 0.0058171$

upper $95 \%$ Mean $\quad-0.00053$

lower $95 \%$ Mean -0.026137

$\mathrm{N}$
Exhibit A8bi.

Distributions Anion=Formate,

Type of Sample $=$ standard, Bottle

ID $=16 \mathrm{ppm}$

Raw Score Difference (ppm)

(M13-M14)

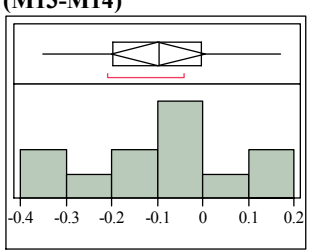

Quantiles

$\begin{array}{llr}100.0 \% & \text { maximum } & 0.1700 \\ 99.5 \% & & 0.1700 \\ 97.5 \% & & 0.1700 \\ 90.0 \% & & 0.1670 \\ 75.0 \% & \text { quartile } & -0.0025 \\ 50.0 \% & \text { median } & -0.0950 \\ 25.0 \% & \text { quartile } & -0.1975 \\ 10.0 \% & & -0.3440 \\ 2.5 \% & & -0.3500 \\ 0.5 \% & -0.3500 \\ 0.0 \% \quad \text { minimum } & -0.3500 \\ \text { Moments } & \\ & \\ \text { Mean } & \\ \text { Std Dev } & -0.0975 \\ \text { Std Err Mean } & 0.1628789 \\ \text { upper 95\% Mean } & 0.0470191 \\ \text { lower 95\% Mean } & -0.2009888 \\ \text { N } & \end{array}$

Exhibit A8bj.

Distributions Anion=Formate,

Type of Sample=standard, Bottle

ID=2ppm

Raw Score Difference (ppm)

(M13-M14)

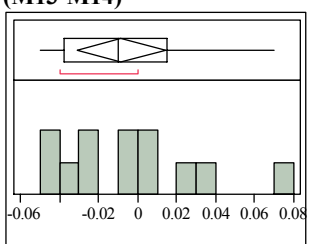

Quantiles

$100.0 \%$ maximum 0.0700

$99.5 \% \quad 0.0700$

$\begin{array}{ll}97.5 \% & 0.0700\end{array}$

$90.0 \% \quad 0.0580$

$75.0 \%$ quartile $\quad 0.0150$

$50.0 \%$ median -0.0100

$25.0 \%$ quartile $\quad-0.0375$

$10.0 \% \quad-0.0500$

$2.5 \% \quad-0.0500$

$0.5 \% \quad-0.0500$

$0.0 \%$ minimum -0.0500

Moments

Mean $\quad-0.008333$

Std Dev $\quad 0.0356328$

Std Err Mean $\quad 0.0102863$

upper $95 \%$ Mean 0.0143067

lower 95\% Mean - 0.030973

$\mathrm{N}$
Exhibit A8bk.

Distributions Anion=Nitrate, Type of Sample=standard, Bottle ID=16ppm

Raw Score Difference (ppm) (M13-M14)

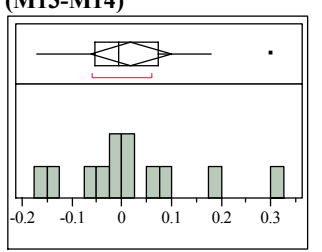

Quantiles

$100.0 \%$ maximum 0.3000

$99.5 \% \quad 0.3000$

$97.5 \% \quad 0.3000$

$90.0 \% \quad 0.2640$

$75.0 \%$ quartile $\quad 0.0750$

$50.0 \%$ median -0.0050

$25.0 \%$ quartile -0.0550

$10.0 \% \quad-0.1580$

$2.5 \% \quad-0.1700$

$0.5 \% \quad-0.1700$

$0.0 \%$ minimum -0.1700

Moments

Mean $\quad 0.0183333$

Std Dev $\quad 0.1279086$

Std Err Mean $\quad 0.036924$

upper 95\% Mean 0.0996026

lower $95 \%$ Mean -0.062936

Exhibit A8bl.

Distributions Anion=Nitrate, Type

of Sample=standard, Bottle

ID $=\mathbf{2 p p m}$

Raw Score Difference (ppm) (M13-M14)

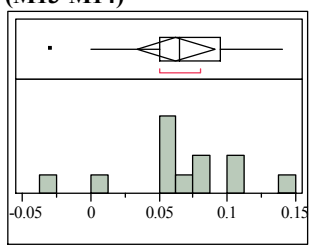

Quantiles

$100.0 \%$ maximum 0.1400

$99.5 \% \quad 0.1400$

$97.5 \% \quad 0.1400$

$90.0 \% \quad 0.1280$

$75.0 \%$ quartile $\quad 0.0950$

$50.0 \%$ median 0.0650

$25.0 \%$ quartile $\quad 0.0500$

$10.0 \% \quad-0.0210$

$2.5 \% \quad-0.0300$

$0.5 \% \quad-0.0300$

$0.0 \%$ minimum -0.0300

Moments

Mean

0.0451513

Std Err Mean $\quad 0.013034$ upper 95\% Mean 0.0911877 lower 95\% Mean 0.0338123

$\mathrm{N}$ 
Exhibit A8bm.

Distributions Anion=Nitrite, Type of Sample $=$ standard, Bottle ID $=16$ ppm

Raw Score Difference (ppm) (M13-M14)

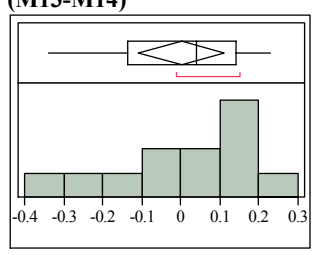

Quantiles

$100.0 \%$ maximum 0.2300

$99.5 \%$

$97.5 \% \quad 0.2300$

$90.0 \% \quad 0.2060$

$75.0 \%$ quartile $\quad 0.1400$

$50.0 \%$ median

$25.0 \%$ quartile $\quad-0.1350$

$10.0 \% \quad-0.3100$

$2.5 \% \quad-0.3400$

$0.5 \% \quad-0.3400$

$0.0 \%$ minimum -0.3400

Moments

Mean $\quad 0.0008333$

Std Dev $\quad 0.1725456$

Std Err Mean $\quad 0.0498096$

upper $95 \%$ Mean 0.1104636

lower 95\% Mean $\quad-0.108797$

$\mathrm{N}$

12

Exhibit A8bn.

Distributions Anion=Nitrite, Type of Sample=standard, Bottle

ID=2ppm

Raw Score Difference (ppm) (M13-M14)

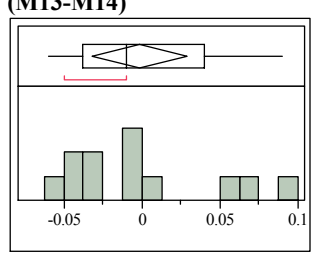

Quantiles

$100.0 \%$ maximum 0.0900

$99.5 \% \quad 0.0900$

$\begin{array}{ll}97.5 \% & 0.0900\end{array}$

$90.0 \% \quad 0.0840$

$75.0 \%$ quartile $\quad 0.0400$

$50.0 \%$ median -0.0100

$25.0 \%$ quartile $\quad-0.0375$

$10.0 \% \quad-0.0570$

$2.5 \% \quad-0.0600$

$0.5 \% \quad-0.0600$

$0.0 \%$ minimum -0.0600

Moments

Mean $\quad-0.001667$

Std Dev $\quad 0.0480215$

Std Err Mean $\quad 0.0138626$ upper $95 \%$ Mean 0.0288447 lower $95 \%$ Mean -0.032178 $\mathrm{N}$
Exhibit A8bo.

Distributions Anion=Oxalate, Type of Sample=standard, Bottle ID=16ppm

Raw Score Difference (ppm) (M13-M14)

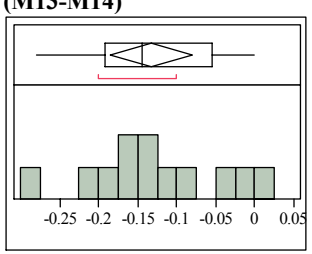

Quantiles

$\begin{array}{lrr}100.0 \% & \text { maximum } & 0.0000 \\ 99.5 \% & & 0.0000 \\ 97.5 \% & & 0.0000 \\ 90.0 \% & & -0.0060 \\ 75.0 \% & \text { quartile } & -0.0550 \\ 50.0 \% & \text { median } & -0.1450 \\ 25.0 \% & \text { quartile } & -0.1925 \\ 10.0 \% & & -0.2590 \\ 2.5 \% & & -0.2800 \\ 0.5 \% & -0.2800 \\ 0.0 \% \quad \text { minimum } & -0.2800 \\ \text { Moments } & \\ & \\ \text { Mean } & \\ \text { Std Dev } & -0.1325 \\ \text { Std Err Mean } & 0.0830252 \\ \text { upper 95\% Mean } & -0.07979 \\ \text { lower 95\% Mean } & -0.185252 \\ \text { N } & \end{array}$

$\mathrm{N}$

Exhibit A8bp.

Distributions Anion=Oxalate, Type of Sample=standard, Bottle

ID=2ppm

Raw Score Difference (ppm) (M13-M14)

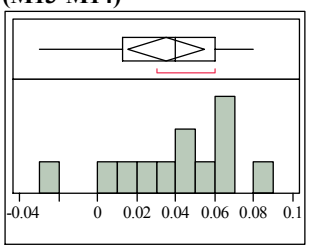

Quantiles

$100.0 \%$ maximum 0.0800

$99.5 \% \quad 0.0800$

$\begin{array}{ll}97.5 \% & 0.0800\end{array}$

$90.0 \% \quad 0.0740$

$75.0 \%$ quartile $\quad 0.0600$

$50.0 \%$ median 0.0400

$25.0 \%$ quartile $\quad 0.0125$

$10.0 \% \quad-0.0210$

$2.5 \% \quad-0.0300$

$0.5 \% \quad-0.0300$

$0.0 \%$ minimum -0.0300

Moments

Mean $\quad 0.035$

Std Dev $\quad 0.0308957$

Std Err Mean $\quad 0.0089188$

upper $95 \%$ Mean 0.0546302

lower 95\% Mean 0.0153698

$\mathrm{N}$
Exhibit A8bq.

Distributions Anion=Phosphate, Type of Sample $=$ standard, Bottle ID $=16 p p m$

Raw Score Difference (ppm)

(M13-M14)

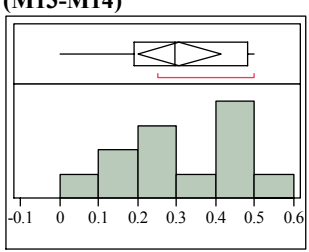

Quantiles

$\begin{array}{lrr}100.0 \% & \text { maximum } & 0.50000 \\ 99.5 \% & & 0.50000 \\ 97.5 \% & & 0.50000 \\ 90.0 \% & & 0.49700 \\ 75.0 \% & \text { quartile } & 0.48000 \\ 50.0 \% & \text { median } & 0.29510 \\ 25.0 \% & \text { quartile } & 0.19000 \\ 10.0 \% & & 0.03030 \\ 2.5 \% & & 0.00000 \\ 0.5 \% & 0.00000 \\ 0.0 \% \quad \text { minimum } & 0.00000 \\ \text { Moments } & \\ & \\ \text { Mean } & 0.3067667 \\ \text { Std Dev } & 0.1691335 \\ \text { Std Err Mean } & 0.0488246 \\ \text { upper 95\% Mean } & 0.414229 \\ \text { lower 95\% Mean } & 0.1993044 \\ \text { N } & \end{array}$

Exhibit A8br

Distributions Anion=Phosphate, Type of Sample=standard, Bottle ID=2ppm

Raw Score Difference (ppm) (M13-M14)

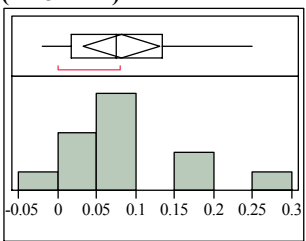

Quantiles

$100.0 \%$ maximum 0.2500

$99.5 \% \quad 0.2500$

$\begin{array}{ll}97.5 \% & 0.2500\end{array}$

$90.0 \% \quad 0.2260$

$75.0 \%$ quartile $\quad 0.1350$

$50.0 \%$ median 0.0750

$25.0 \%$ quartile $\quad 0.0175$

$10.0 \% \quad-0.0140$

$2.5 \% \quad-0.0200$

$0.5 \% \quad-0.0200$

$0.0 \%$ minimum -0.0200

Moments

Mean

$\quad 0.0768854$

Std Err Mean $\quad 0.0221949$

upper 95\% Mean 0.1313506

lower $95 \%$ Mean 0.0336494

$\mathrm{N}$
Exhibit A8bs.

Distributions Anion=Sulfate, Type of Sample $=$ standard, Bottle ID=16ppm

Raw Score Difference (ppm) (M13-M14)

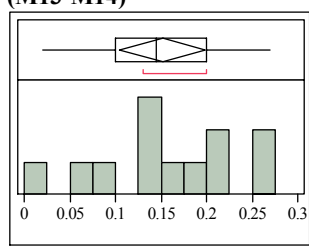

Quantiles

$100.0 \%$ maximum 0.27000

$99.5 \% \quad 0.27000$

$97.5 \% \quad 0.27000$

$90.0 \% \quad 0.26400$

$75.0 \%$ quartile $\quad 0.20000$

$50.0 \%$ median 0.14500

$25.0 \%$ quartile 0.10000

$10.0 \% \quad 0.02900$

$2.5 \% \quad 0.02000$

$0.5 \% \quad 0.02000$

$0.0 \%$ minimum 0.02000

Moments

Mean $\quad 0.1516667$

Std Dev $\quad 0.0746913$

Std Err Mean $\quad 0.0215615$

upper 95\% Mean 0.1991232

lower 95\% Mean 0.1042101

Exhibit A8bt.

Distributions Anion=Sulfate, Type of Sample=standard, Bottle

ID $=\mathbf{2 p p m}$

Raw Score Difference (ppm) (M13-M14)

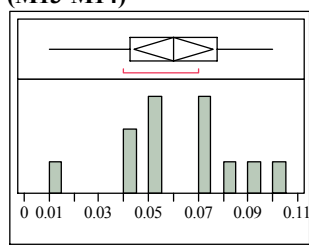

Quantiles

$100.0 \%$ maximum 0.10000

$99.5 \% \quad 0.10000$

$97.5 \% \quad 0.10000$

$90.0 \% \quad 0.09700$

$75.0 \%$ quartile $\quad 0.07750$

$50.0 \%$ median 0.06000

$25.0 \%$ quartile $\quad 0.04250$

$2.5 \% \quad 0.01000$

$0.5 \% \quad 0.01000$

$0.0 \%$ minimum 0.01000

Moments

Mean

Std Dev $\quad 0.0248633$

Std Err Mean $\quad 0.0071774$

upper $95 \%$ Mean 0.0757974

lower 95\% Mean 0.0442026

$\mathrm{N}$ 
Exhibit A9a.

Distributions Anion=Chloride, Type of Sample=standard, Bottle ID $=16 \mathrm{ppm}$

M-13 ICS3000 raw (ppm)

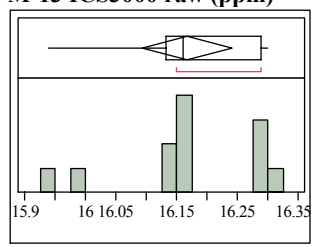

Quantiles

$100.0 \%$ maximum 16.300 $99.5 \% \quad 16.300$

$97.5 \% \quad 16.300$

$90.0 \% \quad 16.297$

$75.0 \%$ quartile $\quad 16.290$

$50.0 \%$ median 16.160

$25.0 \%$ quartile $\quad 16.133$

$10.0 \% \quad 15.955$

$\begin{array}{ll}2.5 \% & 15.940\end{array}$

$0.5 \% \quad 15.940$

$0.0 \%$ minimum 15.940

Moments

Mean $\quad 16.1675$

Std Dev $\quad 0.1161602$

Std Err Mean 0.0335325

upper 95\% Mean 16.241305

lower $95 \%$ Mean 16.093695

$\mathrm{N}$

M-14 ICS3000 raw (ppm)

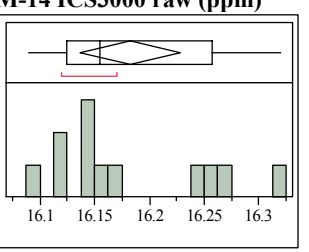

Quantiles

$100.0 \%$ maximum 16.320

$99.5 \% \quad 16.320$

$97.5 \% \quad 16.320$

$90.0 \% \quad 16.305$

$75.0 \%$ quartile 16.258

$50.0 \%$ median 16.155

$25.0 \%$ quartile $\quad 16.125$

$10.0 \% \quad 16.099$

$2.5 \% \quad 16.090$

$0.5 \% \quad 16.090$

$0.0 \%$ minimum 16.090

Moments

Mean $\quad 16.1825$

Std Dev $\quad 0.0731282$

Std Err Mean $\quad 0.0211103$

upper 95\% Mean 16.228963

lower 95\% Mean 16.136037

$\mathrm{N}$
Exhibit A9b.

Distributions Anion=Chloride,

Type of Sample=standard, Bottle

ID=2ppm

M-13 ICS3000 raw (ppm)

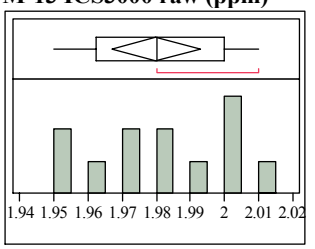

Quantiles

$100.0 \%$ maximum 2.0100

$99.5 \% \quad 2.0100$

$97.5 \% \quad 2.0100$

$90.0 \% \quad 2.0070$

$75.0 \%$ quartile 2.0000

$50.0 \%$ median 1.9800

$25.0 \%$ quartile 1.9625

$10.0 \% \quad 1.9500$

$\begin{array}{ll}2.5 \% & 1.9500\end{array}$

$0.5 \% \quad 1.9500$

$0.0 \%$ minimum 1.9500

Moments

Mean 1.98

Std Dev $\quad 0.0204495$

Std Err Mean $\quad 0.0059033$

upper 95\% Mean 1.992993

lower 95\% Mean 1.967007

$\mathrm{N}$

12

M-14 ICS3000 raw (ppm)

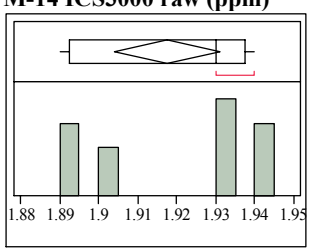

Quantiles

$100.0 \%$ maximum 1.9400

$99.5 \% \quad 1.9400$

$97.5 \% \quad 1.9400$

$90.0 \% \quad 1.9400$

$75.0 \%$ quartile 1.9375

$50.0 \%$ median

$25.0 \%$ quartile 1.8925

$10.0 \% \quad 1.8900$

$2.5 \% \quad 1.8900$

$0.5 \% \quad 1.8900$

$0.0 \%$ minimum 1.8900

Moments

Mean $\quad 1.9175$

Std Dev $\quad 0.0213733$

Std Err Mean $\quad 0.0061699$

upper 95\% Mean 1.93108

lower 95\% Mean 1.90392

$\mathrm{N}$

12
Exhibit A9c.

Distributions Anion=Fluoride,

Type of Sample $=$ standard, Bottle

ID $=16 \mathrm{ppm}$

M-13 ICS3000 raw (ppm)

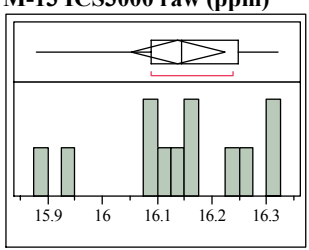

Quantiles

$100.0 \%$ maximum 16.320

$99.5 \% \quad 16.320$

$97.5 \% \quad 16.320$

$90.0 \% \quad 16.317$

$75.0 \%$ quartile 16.248

$50.0 \%$ median 16.145

$25.0 \%$ quartile $\quad 16.090$

$10.0 \% \quad 15.898$

$\begin{array}{ll}10.0 \% & 15.880\end{array}$

$0.5 \% \quad 15.880$

$0.0 \%$ minimum 15.880

Moments

Mean $\quad 16.138333$

Std Dev $\quad 0.134085$

Std Err Mean $\quad 0.038707$

upper 95\% Mean 16.223527

lower $95 \%$ Mean $\quad 16.05314$

$\mathrm{N}$

12

M-14 ICS3000 raw (ppm)

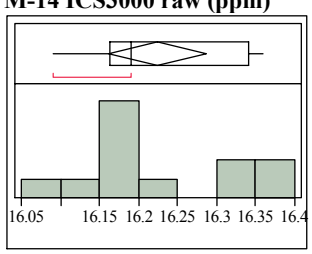

Quantiles

$100.0 \%$ maximum 16.360

$99.5 \% \quad 16.360$

$97.5 \% \quad 16.360$

$90.0 \% \quad 16.360$

$75.0 \%$ quartile $\quad 16.340$

$50.0 \%$ median 16.190

$25.0 \%$ quartile 16.163

$10.0 \% \quad 16.093$

$2.5 \% \quad 16.090$

$0.5 \% \quad 16.090$

$0.0 \%$ minimum 16.090

Moments

Mean $\quad 16.224167$

Std Dev $\quad 0.0997687$

Std Err Mean $\quad 0.0288007$

upper $95 \%$ Mean 16.287557

lower 95\% Mean 16.160777

$\mathrm{N}$
Exhibit A9d.

Distributions Anion=Fluoride,

Type of Sample=standard, Bottle

ID=2ppm

M-13 ICS3000 raw (ppm)

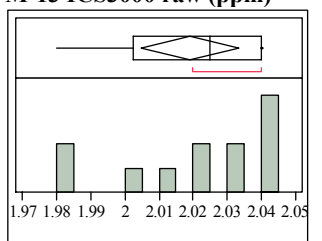

Quantiles

$100.0 \%$ maximum 2.0400

$99.5 \% \quad 2.0400$

$97.5 \% \quad 2.0400$

$90.0 \% \quad 2.0400$

$75.0 \%$ quartile 2.0400

$50.0 \%$ median 2.0250

$25.0 \%$ quartile 2.0025

$10.0 \% \quad 1.9800$

$\begin{array}{ll}10.0 \% & 1.9800\end{array}$

$0.5 \% \quad 1.9800$

$0.0 \%$ minimum 1.9800

Moments

Mean 2.0191667

Std Dev $\quad 0.0223437$

Std Err Mean 0.0064501

upper 95\% Mean 2.0333632

lower 95\% Mean 2.0049701

$\mathrm{N}$

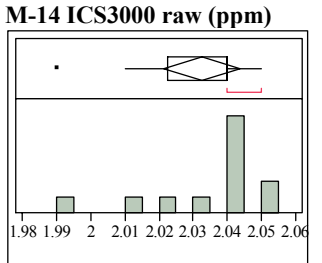

Quantiles

$100.0 \%$ maximum 2.0500

$99.5 \% \quad 2.0500$

$97.5 \% \quad 2.0500$

$90.0 \% \quad 2.0500$

$75.0 \%$ quartile 2.0400

$50.0 \%$ median 2.0400

$25.0 \%$ quartile 2.0225

$10.0 \% \quad 1.9960$

$2.5 \% \quad 1.9900$

$0.5 \% \quad 1.9900$

$0.0 \%$ minimum 1.9900

Moments

Mean 2.0325

Std Dev $\quad 0.0176455$

Std Err Mean 0.0050938

upper 95\% Mean 2.0437114

lower 95\% Mean 2.0212886

$\mathrm{N}$ 
Exhibit A9e.

Distributions Anion=Formate, Type of Sample=standard, Bottle ID $=16 \mathrm{ppm}$

M-13 ICS3000 raw (ppm)

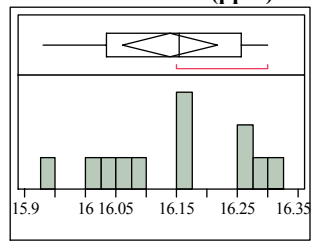

Quantiles

$100.0 \%$ maximum 16.300

$99.5 \% \quad 16.300$

$97.5 \% \quad 16.300$

$90.0 \% \quad 16.297$

$75.0 \%$ quartile 16.258

$50.0 \%$ median 16.155

$25.0 \%$ quartile $\quad 16.035$

$10.0 \% \quad 15.951$

$\begin{array}{ll}10.0 \% & 15.930\end{array}$

$0.5 \% \quad 15.930$

$0.0 \%$ minimum 15.930

Moments

Mean $\quad 16.139167$

Std Dev $\quad 0.1219134$

Std Err Mean $\quad 0.0351934$

upper 95\% Mean 16.216627

lower $95 \%$ Mean 16.061707

$\mathrm{N}$

12

M-14 ICS3000 raw (ppm)

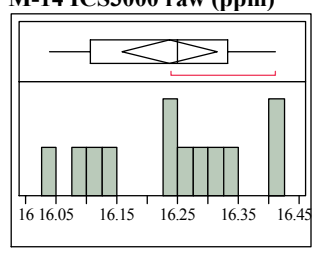

Quantiles

$100.0 \%$ maximum 16.410

$99.5 \% \quad 16.410$

$97.5 \% \quad 16.410$

$90.0 \% \quad 16.410$

$75.0 \%$ quartile $\quad 16.333$

$50.0 \%$ median 16.250

$25.0 \%$ quartile 16.108

$10.0 \% \quad 16.055$

$2.5 \% \quad 16.040$

$0.5 \% \quad 16.040$

$0.0 \%$ minimum 16.040

Moments

Mean $\quad 16.236667$

Std Dev $\quad 0.1239012$

Std Err Mean $\quad 0.0357672$

upper 95\% Mean 16.31539

lower 95\% Mean 16.157944

$\mathrm{N}$
Exhibit A9f.

Distributions Anion=Formate,

Type of Sample=standard, Bottle

ID=2ppm

M-13 ICS3000 raw (ppm)

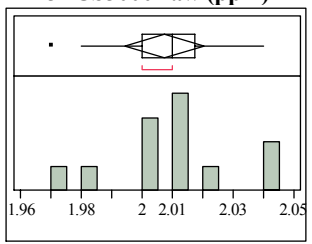

Quantiles

$100.0 \%$ maximum 2.0400

$99.5 \% \quad 2.0400$

$97.5 \% \quad 2.0400$

$90.0 \% \quad 2.0400$

$75.0 \%$ quartile 2.0175

$50.0 \%$ median 2.0100

$25.0 \%$ quartile 2.0000

$10.0 \% \quad 1.9730$

$\begin{array}{ll}2.5 \% & 1.9700\end{array}$

$0.5 \% \quad 1.9700$

$0.0 \%$ minimum 1.9700

Moments

Mean 2.0075

Std Dev $\quad 0.020505$

Std Err Mean $\quad 0.0059193$

upper 95\% Mean 2.0205282

lower $95 \%$ Mean 1.9944718

$\mathrm{N}$

12

M-14 ICS3000 raw (ppm)

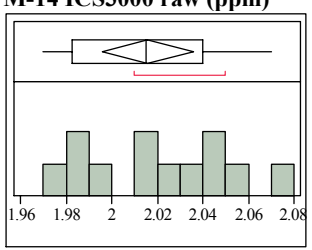

Quantiles

$100.0 \%$ maximum 2.0700

$99.5 \% \quad 2.0700$

$97.5 \% \quad 2.0700$

$90.0 \% \quad 2.0640$

$75.0 \%$ quartile 2.0400

$50.0 \%$ median 2.0150

$25.0 \%$ quartile $\quad 1.9825$

$10.0 \% \quad 1.9730$

$2.5 \% \quad 1.9700$

$0.5 \% \quad 1.9700$

$0.0 \%$ minimum 1.9700

Moments

Mean $\quad 2.0158333$

Std Dev $\quad 0.0314667$

Std Err Mean $\quad 0.0090836$

upper $95 \%$ Mean 2.0358263

lower $95 \%$ Mean 1.9958404

$\mathrm{N}$
Exhibit A9g.

Distributions Anion=Nitrate, Type of Sample=standard, Bottle ID $=16 \mathrm{ppm}$

M-13 ICS3000 raw (ppm)

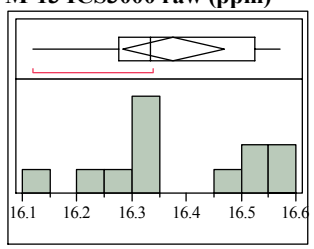

Quantiles

$100.0 \%$ maximum 16.570

$99.5 \% \quad 16.570$

$97.5 \% \quad 16.570$

$90.0 \% \quad 16.567$

$75.0 \%$ quartile 16.525

$50.0 \%$ median 16.335

$25.0 \%$ quartile $\quad 16.278$

$10.0 \% \quad 16.147$

$\begin{array}{ll}10.0 \% & 16.147 \\ 2.5 \% & 16.120\end{array}$

$0.5 \% \quad 16.120$

$0.0 \%$ minimum 16.120

Moments

Mean $\quad 16.375833$

Std Dev $\quad 0.1462537$

Std Err Mean $\quad 0.0422198$

upper 95\% Mean 16.468759

lower $95 \%$ Mean 16.282908

$\mathrm{N}$

12

M-14 ICS3000 raw (ppm)

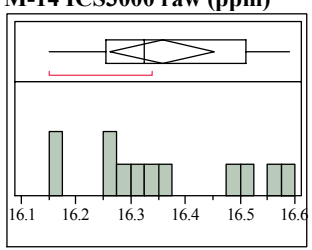

Quantiles

$100.0 \%$ maximum 16.590

$99.5 \% \quad 16.590$

$97.5 \% \quad 16.590$

$90.0 \% \quad 16.584$

$75.0 \%$ quartile $\quad 16.510$

$50.0 \%$ median 16.325

$25.0 \%$ quartile $\quad 16.255$

$10.0 \%$ quartile 16.153

$2.5 \% \quad 16.150$

$0.5 \% \quad 16.150$

$0.0 \%$ minimum 16.150

Moments

Mean

Std Dev $\quad 0.1504614$

upper 95\% Mean 16.453099

lower $95 \%$ Mean 16.261901
Exhibit A9h.

Distributions Anion=Nitrate, Type of Sample=standard, Bottle

ID=2ppm

M-13 ICS3000 raw (ppm)

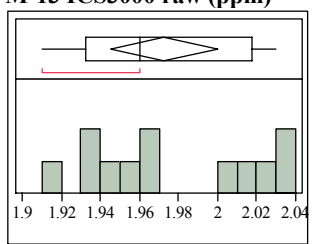

Quantiles

$100.0 \%$ maximum 2.0300

$99.5 \% \quad 2.0300$

$97.5 \% \quad 2.0300$

$90.0 \% \quad 2.0300$

$75.0 \%$ quartile 2.0175

$50.0 \%$ median $\quad 1.9600$

$25.0 \%$ quartile $\quad 1.9325$

$10.0 \% \quad 1.9160$

$\begin{array}{ll}2.5 \% & 1.9100 \\ 0.5 \% & 1.9100\end{array}$

$0.5 \% \quad 1.9100$

$0.0 \%$ minimum 1.9100

Moments

Mean $\quad 1.9725$

Std Dev $\quad 0.0430908$

Std Err Mean 0.0124392

upper 95\% Mean 1.9998786

lower 95\% Mean 1.9451214

$\mathrm{N}$

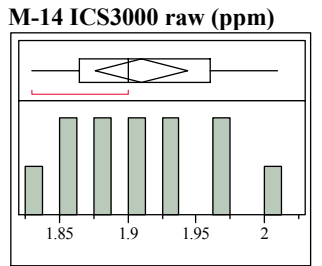

Quantiles

$100.0 \%$ maximum 2.0100

$99.5 \% \quad 2.0100$

$97.5 \%$

$90.0 \% \quad 1.9980$

$75.0 \%$ quartile 1.9600

$50.0 \%$ median 1.9000

$25.0 \%$ quartile 1.8650

$10.0 \% \quad 1.8390$

$2.5 \% \quad 1.8300$

$0.5 \% \quad 1.8300$

$0.0 \%$ minimum 1.8300

Moments

Mean

Std Err Mean $\quad 0.0154233$

upper 95\% Mean 1.9439465

lower 95\% Mean 1.8760535

$\mathrm{N}$ 
Exhibit A9i.

Distributions Anion=Nitrite, Type of Sample $=$ standard, Bottle ID=16ppm

M-13 ICS3000 raw (ppm)

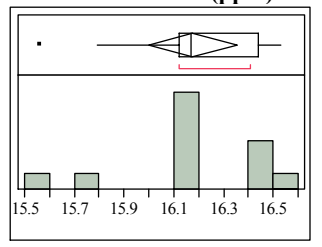

Quantiles

$100.0 \%$ maximum 16.530

$99.5 \%$

$97.5 \%$

$90.0 \%$

$75.0 \%$ quartile $\quad 16.440$

$50.0 \%$ median 16.170

$25.0 \%$ quartile $\quad 16.123$

$10.0 \% \quad 15.629$

$\begin{array}{ll}2.5 \% & 15.560\end{array}$

$0.5 \% \quad 15.560$

$0.0 \%$ minimum 15.560

Moments

Mean $\quad 16.176667$

Std Dev $\quad 0.2812742$

Std Err Mean $\quad 0.0811969$

upper 95\% Mean 16.35538

lower $95 \%$ Mean 15.997954

$\mathrm{N}$

M-14 ICS3000 raw (ppm)

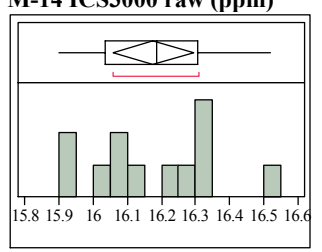

Quantiles

$100.0 \%$ maximum 16.520

$99.5 \% \quad 16.520$

$97.5 \% \quad 16.520$

$90.0 \% \quad 16.466$

$75.0 \%$ quartile 16.308

$50.0 \%$ median 16.185

$25.0 \%$ quartile $\quad 16.038$

$10.0 \% \quad 15.906$

$2.5 \% \quad 15.900$

$0.5 \% \quad 15.900$

$0.0 \%$ minimum 15.900

Moments

Mean $\quad 16.175833$

Std Dev $\quad 0.1856908$

Std Err Mean $\quad 0.0536043$

upper $95 \%$ Mean 16.293816

lower 95\% Mean 16.057851

$\mathrm{N}$
Exhibit A9j.

Distributions Anion=Nitrite, Type of Sample $=$ standard, Bottle ID=2ppm

M-13 ICS3000 raw (ppm)

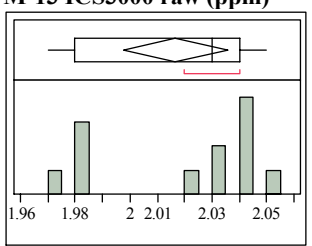

Quantiles

$100.0 \%$ maximum 2.0500

$99.5 \% \quad 2.0500$

$97.5 \% \quad 2.0500$

$90.0 \% \quad 2.0470$

$75.0 \%$ quartile 2.0400

$50.0 \%$ median 2.0300

$25.0 \%$ quartile $\quad 1.9800$

$10.0 \% \quad 1.9730$

$\begin{array}{ll}2.5 \% & 1.9700\end{array}$

$0.5 \% \quad 1.9700$

$0.0 \%$ minimum 1.9700

Moments

Mean $\quad 2.0166667$

Std Dev $\quad 0.0299495$

Std Err Mean $\quad 0.0086457$

upper 95\% Mean 2.0356956

lower $95 \%$ Mean 1.9976377

$\mathrm{N}$

12

M-14 ICS3000 raw (ppm)

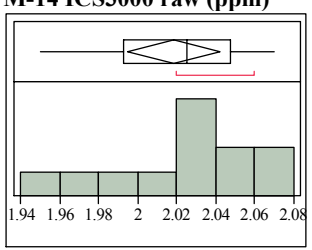

Quantiles

$100.0 \%$ maximum 2.0700

$99.5 \% \quad 2.0700$

$97.5 \% \quad 2.0700$

$90.0 \% \quad 2.0670$

$75.0 \%$ quartile 2.0475

$50.0 \%$ median

$25.0 \%$ quartile 1.9925

$10.0 \% \quad 1.9530$

$2.5 \% \quad 1.9500$

$0.5 \% \quad 1.9500$

$0.0 \%$ minimum 1.9500

Moments

Mean $\quad 2.0183333$

Std Dev $\quad 0.0373761$

Std Err Mean $\quad 0.0107895$

upper $95 \%$ Mean 2.0420809

lower 95\% Mean 1.9945857

N
Exhibit A9k.

Exhibit A91.

Distributions Anion=Oxalate, Type Distributions Anion=Oxalate, Type of Sample=standard, Bottle ID $=16 \mathrm{ppm}$

M-13 ICS3000 raw (ppm)

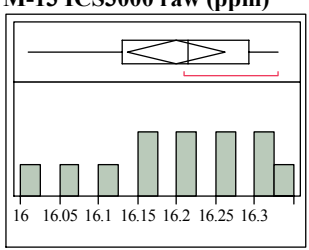

Quantiles

$100.0 \%$ maximum 16.330

$99.5 \% \quad 16.330$

$97.5 \% \quad 16.330$

$90.0 \% \quad 16.321$

$75.0 \%$ quartile 16.293

$50.0 \%$ median 16.215

$25.0 \%$ quartile $\quad 16.130$

$10.0 \% \quad 16.028$

$\begin{array}{ll}10.0 \% & 16.028 \\ 2.5 \% & 16.010\end{array}$

$0.5 \% \quad 16.010$

$0.0 \%$ minimum 16.010

Moments

Mean 16.2

Std Dev $\quad 0.098811$

Std Err Mean $\quad 0.0285243$

upper 95\% Mean 16.262782

lower $95 \%$ Mean 16.137218

$\mathrm{N}$

12

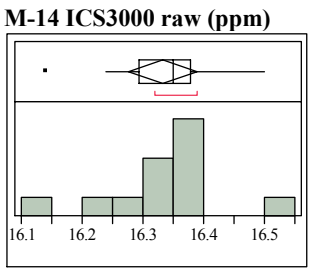

Quantiles

$100.0 \%$ maximum 16.500

$99.5 \% \quad 16.500$

$97.5 \% \quad 16.500$

$90.0 \% \quad 16.467$

$75.0 \%$ quartile $\quad 16.378$

$50.0 \%$ median 16.350

$25.0 \%$ quartile $\quad 16.293$

$\begin{array}{ll}25.0 \% & 16.170\end{array}$

$2.5 \% \quad 16.140$

$0.5 \% \quad 16.140$

$0.0 \%$ minimum 16.140

Moments

Mean

Std Dev $\quad 0.0880212$

upper $95 \%$ Mean 16.388426

lower 95\% Mean 16.276574

$\mathrm{N}$

12 of Sample=standard, Bottle

ID $=2$ ppm

M-13 ICS3000 raw (ppm)

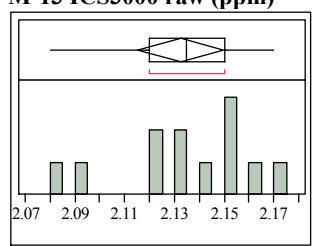

Quantiles

$100.0 \%$ maximum 2.1700

$97.5 \% \quad 2.1700$

$90.0 \% \quad 2.1670$

$75.0 \%$ quartile 2.1500

$50.0 \%$ median 2.1350

$25.0 \%$ quartile 2.1200

$10.0 \% \quad 2.0830$

$2.5 \% \quad 2.0800$

$0.5 \% \quad 2.0800$

$0.0 \%$ minimum 2.0800

Moments

Mean $\quad 2.1325$

Std Dev $\quad 0.0270101$

Std Err Mean $\quad 0.0077971$

upper 95\% Mean 2.1496614

lower 95\% Mean 2.1153386

$\mathrm{N}$

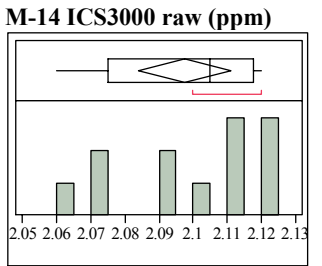

Quantiles

$100.0 \%$ maximum 2.1200

$99.5 \% \quad 2.1200$

$97.5 \%$

$90.0 \% \quad 2.1200$

$75.0 \%$ quartile 2.1175

$50.0 \%$ median 2.1050

$25.0 \%$ quartile 2.0750

$10.0 \% \quad 2.0630$

$2.5 \% \quad 2.0600$

$0.5 \% \quad 2.0600$

$0.0 \%$ minimum 2.0600

Moments

Mean $\quad 2.0975$

Std Dev $\quad 0.0213733$

Std Err Mean $\quad 0.0061699$

upper $95 \%$ Mean 2.11108

lower 95\% Mean 2.08392

$\mathrm{N}$
$99.5 \% \quad 2.1700$ 
Exhibit A9m.

Distributions Anion=Phosphate, Type of Sample $=$ standard, Bottle ID $=16 \mathrm{ppm}$

M-13 ICS3000 raw (ppm)

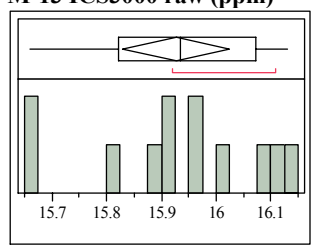

Quantiles

$100.0 \%$ maximum 16.130

$99.5 \% \quad 16.130$

$97.5 \% \quad 16.130$

$90.0 \% \quad 16.124$

$75.0 \%$ quartile 16.073

$50.0 \%$ median 15.935

$25.0 \%$ quartile $\quad 15.823$

$10.0 \% \quad 15.663$

$\begin{array}{ll}2.5 \% & 15.660 \\ 0.5 \% & 15.660\end{array}$

$0.5 \% \quad 15.660$

$0.0 \%$ minimum 15.660

Moments

Mean $\quad 15.926667$

Std Dev $\quad 0.1556414$

Std Err Mean $\quad 0.0449298$

upper 95\% Mean 16.025556

lower $95 \%$ Mean 15.827777

$\mathrm{N}$

M-14 ICS3000 raw (ppm)

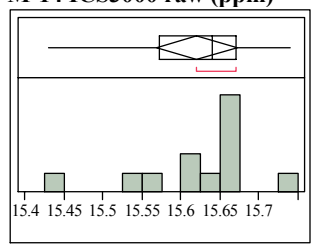

Quantiles

$100.0 \%$ maximum 15.740

$99.5 \% \quad 15.740$

$97.5 \% \quad 15.740$

$90.0 \% \quad 15.719$

$75.0 \%$ quartile $\quad 15.670$

$50.0 \%$ median 15.640

$25.0 \%$ quartile $\quad 15.572$

$\begin{array}{ll}10.0 \% & 15.460 \\ 2.5 \% & 15.430\end{array}$

$2.5 \% \quad 15.430$

$0.5 \% \quad 15.430$

$0.0 \%$ minimum 15.430

Moments

Mean $\quad 15.6199$

Std Dev $\quad 0.0811984$

Std Err Mean $\quad 0.0234399$

upper 95\% Mean 15.671491

lower 95\% Mean 15.568309

$\mathrm{N}$
Exhibit A9n.

Distributions Anion=Phosphate, Type of Sample=standard, Bottle ID=2ppm

M-13 ICS3000 raw (ppm)

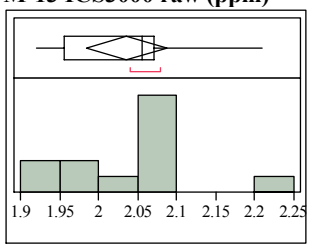

Quantiles

$100.0 \%$ maximum 2.2100

$99.5 \% \quad 2.2100$

$97.5 \% \quad 2.2100$

$90.0 \% \quad 2.1710$

$75.0 \%$ quartile 2.0700

$50.0 \%$ median 2.0550

$25.0 \%$ quartile $\quad 1.9550$

$10.0 \% \quad 1.9260$

$\begin{array}{ll}2.5 \% & 1.9200\end{array}$

$0.5 \% \quad 1.9200$

$0.0 \%$ minimum 1.9200

Moments

Mean 2.0358333

Std Dev $\quad 0.0802789$

Std Err Mean $\quad 0.0231745$

upper 95\% Mean 2.0868401

lower 95\% Mean 1.9848266

$\mathrm{N}$

12

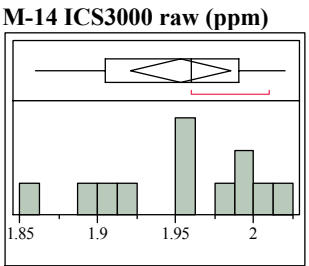

Quantiles

$100.0 \%$ maximum 2.0200

$99.5 \% \quad 2.0200$

$97.5 \% \quad 2.0200$

$90.0 \% \quad 2.0170$

$75.0 \%$ quartile 1.9900

$50.0 \%$ median

$25.0 \%$ quartile 1.9050

$10.0 \% \quad 1.8690$

$2.5 \% \quad 1.8600$

$0.5 \% \quad 1.8600$

$0.0 \%$ minimum 1.8600

Moments

Mean $\quad 1.9533333$

Std Dev $\quad 0.0503322$

Std Err Mean $\quad 0.0145297$

upper 95\% Mean 1.9853129

lower $95 \%$ Mean 1.9213538

$\mathrm{N}$
Exhibit A90.

Distributions Anion=Sulfate, Type of Sample=standard, Bottle ID=16ppm

M-13 ICS3000 raw (ppm)

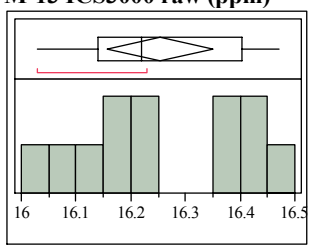

Quantiles

$100.0 \%$ maximum 16.470

$99.5 \% \quad 16.470$

$97.5 \% \quad 16.470$

$90.0 \% \quad 16.461$

$75.0 \%$ quartile 16.403

$50.0 \%$ median 16.220

$25.0 \%$ quartile $\quad 16.140$

$10.0 \% \quad 16.036$

$\begin{array}{ll}10.0 \% & 16.036 \\ 2.5 \% & 16.030\end{array}$

$0.5 \% \quad 16.030$

$0.0 \%$ minimum 16.030

Moments

Mean $\quad 16.254167$

Std Dev $\quad 0.151625$

Std Err Mean $\quad 0.0437704$

upper 95\% Mean 16.350505

lower $95 \%$ Mean 16.157829

$\mathrm{N}$

M-14 ICS3000 raw (ppm)

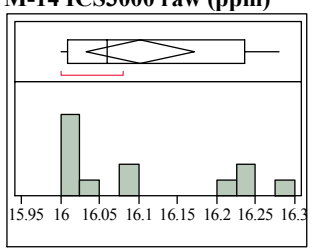

Quantiles

$100.0 \%$ maximum 16.280

$99.5 \% \quad 16.280$

$97.5 \% \quad 16.280$

$90.0 \% \quad 16.268$

$75.0 \%$ quartile 16.235

$50.0 \%$ median 16.060

$25.0 \%$ quartile $\quad 16.010$

$\begin{array}{ll}25.0 \% & 16.000\end{array}$

$2.5 \% \quad 16.000$

$0.5 \% \quad 16.000$

$0.0 \%$ minimum 16.000

Moments

Mean

Std Dev $\quad 0.1098035$

upper $95 \%$ Mean 16.172266

lower 95\% Mean 16.032734

$\mathrm{N}$

12
Exhibit A9p.

Distributions Anion=Sulfate, Type of Sample=standard, Bottle ID=2ppm

M-13 ICS3000 raw (ppm)

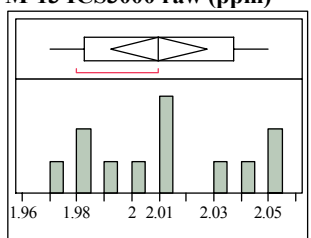

Quantiles

$100.0 \%$ maximum 2.0500

$99.5 \% \quad 2.0500$

$97.5 \% \quad 2.0500$

$90.0 \% \quad 2.0500$

$75.0 \%$ quartile 2.0375

$50.0 \%$ median 2.0100

$25.0 \%$ quartile $\quad 1.9825$

$10.0 \% \quad 1.9730$

$\begin{array}{ll}2.5 \% & 1.9700\end{array}$

$0.5 \% \quad 1.9700$

$0.0 \%$ minimum 1.9700

Moments

Mean 2.01

Std Dev $\quad 0.027634$

Std Err Mean $\quad 0.0079772$

upper 95\% Mean 2.0275578

lower 95\% Mean 1.9924422

$\mathrm{N}$

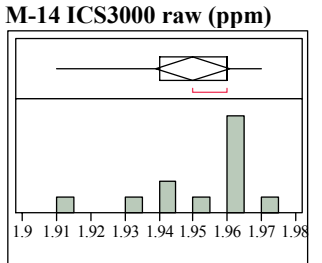

Quantiles

$100.0 \%$ maximum 1.9700

$99.5 \% \quad 1.9700$

$97.5 \%$

$90.0 \% \quad 1.9670$

$75.0 \%$ quartile 1.9600

$50.0 \%$ median 1.9600

$25.0 \%$ quartile 1.9400

$10.0 \% \quad 1.9160$

$2.5 \% \quad 1.9100$

$0.5 \% \quad 1.9100$

$0.0 \%$ minimum 1.9100

Moments

Mean

Std Err Mean $\quad 0.0049237$

upper 95\% Mean 1.9608369

lower 95\% Mean 1.9391631

$\mathrm{N}$ 
Exhibit A10a.

Distributions Type $=$ Simulated

Sample Crosscheck Data 5000X

Dilution, Simulant=SME, Known

Concentration $(\mathrm{ppm})=1$,

Anion=Chloride, ICS-3000

Instrument $=\mathrm{M}-13$

Measurement (ppm)

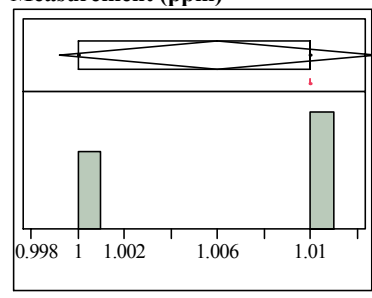

Quantiles

$100.0 \%$ maximum 1.0100

$99.5 \% \quad 1.0100$

$97.5 \% \quad 1.0100$

$90.0 \% \quad 1.0100$

$75.0 \%$ quartile $\quad 1.0100$

$50.0 \%$ median $\quad 1.0100$

$25.0 \%$ quartile $\quad 1.0000$

$10.0 \% \quad 1.0000$

$2.5 \% \quad 1.0000$

$0.5 \% \quad 1.0000$

$0.0 \%$ minimum 1.0000

Moments

Mean $\quad 1.006$

Std Dev $\quad 0.0054772$

Std Err Mean $\quad 0.0024495$

upper $95 \%$ Mean 1.0128009

lower 95\% Mean 0.9991991

$\mathrm{N}$

Instrument $=\mathrm{M}-14$

Measurement (ppm)

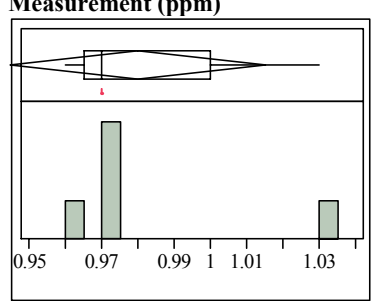

Quantiles

$100.0 \%$ maximum 1.0300

$99.5 \% \quad 1.0300$

$\begin{array}{ll}97.5 \% & 1.0300\end{array}$

$90.0 \% \quad 1.0300$

$75.0 \%$ quartile $\quad 1.0000$

$50.0 \%$ median 0.9700

$25.0 \%$ quartile 0.9650

$10.0 \% \quad 0.9600$

$2.5 \% \quad 0.9600$

$0.5 \% \quad 0.9600$

$0.0 \%$ minimum 0.9600

Moments

Mean

Std Dev $\quad 0.0282843$

Std Err Mean $\quad 0.0126491$

upper 95\% Mean 1.0151196

lower 95\% Mean 0.9448804
Exhibit A10b.

Distributions Type $=$ Simulated

Sample Crosscheck Data 5000X

Dilution, Simulant=SME, Known

Concentration $(\mathrm{ppm})=1$,

Anion=Fluoride, ICS-3000

Instrument=M-13

Measurement (ppm)

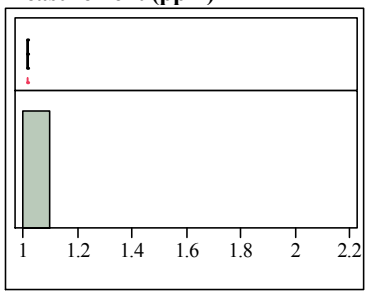

Quantiles

$100.0 \%$ maximum 1.0200

$\begin{array}{ll}9.5 \% & 1.0200 \\ 97.5 \% & 1.0200\end{array}$

$90.0 \% \quad 1.0200$

$75.0 \%$ quartile $\quad 1.0200$

$50.0 \%$ median 1.0200

$25.0 \%$ quartile $\quad 1.0200$

$10.0 \% \quad 1.0200$

$\begin{array}{ll}10.0 \% & 1.0200\end{array}$

$0.5 \% \quad 1.0200$

$0.0 \%$ minimum 1.0200

Moments

Mean

Std Dev

Std Err Mean
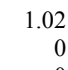

upper $95 \%$ Mean 1.02

lower $95 \%$ Mean 1.02

$\mathrm{N}$

Instrument=M-14

Measurement (ppm)

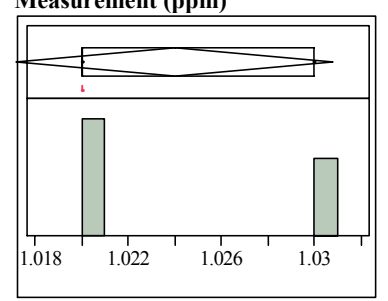

Quantiles

$100.0 \%$ maximum 1.0300

$99.5 \% \quad 1.0300$

$\begin{array}{ll}97.5 \% & 1.0300\end{array}$

$90.0 \% \quad 1.0300$

$75.0 \%$ quartile $\quad 1.0300$

$50.0 \%$ median 1.0200

$25.0 \%$ quartile 1.0200

$10.0 \% \quad 1.0200$

$2.5 \% \quad 1.0200$

$0.5 \% \quad 1.0200$

$0.0 \%$ minimum 1.0200

Moments

Mean

$\quad 0.0054772$

Std Err Mean $\quad 0.0024495$

upper $95 \%$ Mean 1.0308009

lower 95\% Mean 1.0171991
Exhibit A10c.

Distributions Type $=$ Simulated

Sample Crosscheck Data 5000X

Dilution, Simulant=SME, Known

Concentration $(\mathrm{ppm})=1$,

Anion=Nitrite, ICS-3000

Instrument $=\mathbf{M}-\mathbf{1 3}$

Measurement (ppm)

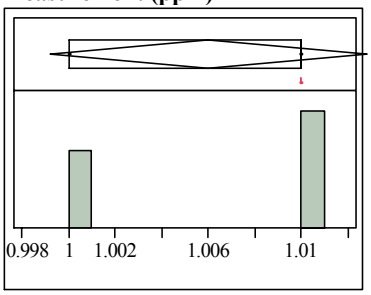

Quantiles

$100.0 \%$ maximum 1.0100

$99.5 \% \quad 1.0100$

$97.5 \% \quad 1.0100$

$90.0 \% \quad 1.0100$

$75.0 \%$ quartile $\quad 1.0100$

$50.0 \%$ median 1.0100

$25.0 \%$ quartile 1.0000

$10.0 \% \quad 1.0000$

$2.5 \% \quad 1.0000$

$0.5 \% \quad 1.0000$

$0.0 \%$ minimum 1.0000

Moments

Mean $\quad 1.006$

Std Dev $\quad 0.0054772$

Std Err Mean $\quad 0.0024495$ upper 95\% Mean 1.0128009 lower 95\% Mean 0.9991991

$\mathrm{N}$

Instrument $=\mathrm{M}-14$

Measurement (ppm)

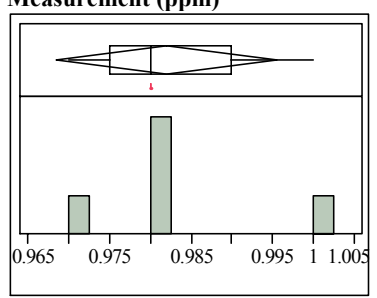

Quantiles

$100.0 \%$ maximum 1.0000

$99.5 \% \quad 1.0000$

$\begin{array}{ll}97.5 \% & 1.0000 \\ 90.0 \% & 1.0000\end{array}$

$90.0 \% \quad 1.0000$

$75.0 \%$ quartile $\quad 0.9900$

$50.0 \%$ median 0.9800

$25.0 \%$ quartile $\quad 0.9750$

$10.0 \% \quad 0.9700$

$2.5 \% \quad 0.9700$

$0.5 \% \quad 0.9700$

$0.0 \%$ minimum 0.9700

Moments

Mean

Std Dev $\quad 0.0109545$

Std Err Mean $\quad 0.004899$

upper $95 \%$ Mean 0.9956017

lower $95 \%$ Mean 0.9683983
Exhibit A10d.

Distributions $\mathrm{Type}=$ Simulated

Sample Crosscheck Data 5000X

Dilution, Simulant=SME, Known

Concentration $(\mathrm{ppm})=1$,

Anion $=$ Oxalate, ICS-3000

Instrument $=\mathrm{M}-13$

Measurement (ppm)

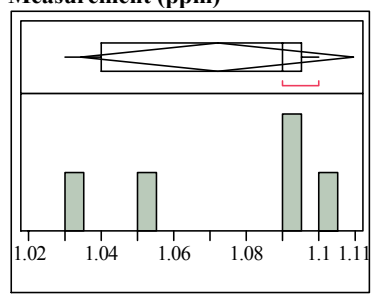

Quantiles

$100.0 \%$ maximum 1.1000

$99.5 \% \quad 1.1000$

$97.5 \% \quad 1.1000$

$90.0 \% \quad 1.1000$

$75.0 \%$ quartile $\quad 1.0950$

$50.0 \%$ median 1.0900

$25.0 \%$ quartile 1.0400

$10.0 \% \quad 1.0300$

$2.5 \% \quad 1.0300$

$0.5 \% \quad 1.0300$

$0.0 \%$ minimum 1.0300

Moments

Mean

Std Dev $\quad 0.0303315$

Std Err Mean $\quad 0.0135647$

upper 95\% Mean 1.1096615

lower 95\% Mean 1.0343385

$\mathrm{N}$

Instrument $=\mathrm{M}-14$

Measurement (ppm)

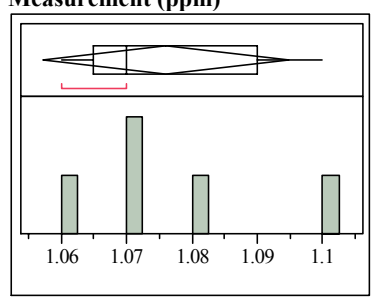

Quantiles

$100.0 \%$ maximum 1.1000

$99.5 \% \quad 1.1000$

$\begin{array}{ll}97.5 \% & 1.1000\end{array}$

$90.0 \% \quad 1.1000$

$75.0 \%$ quartile $\quad 1.0900$

$50.0 \%$ median 1.0700

$25.0 \%$ quartile $\quad 1.0650$

$10.0 \% \quad 1.0600$

$2.5 \% \quad 1.0600$

$\begin{array}{ll}0.5 \% & 1.0600\end{array}$

$0.0 \%$ minimum 1.0600

Moments

Mean $\quad 1.076$

Std Dev $\quad 0.0151658$

Std Err Mean $\quad 0.0067823$

upper 95\% Mean 1.0948308

lower 95\% Mean 1.0571692

N 
Exhibit A10e.

Distributions Type $=$ Simulated

Sample Crosscheck Data 5000X

Dilution, Simulant=SME, Known

Concentration $(\mathrm{ppm})=1$,

Anion=Phosphate, ICS-3000

Instrument $=\mathrm{M}-13$

Measurement (ppm)

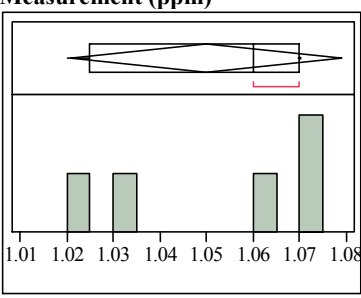

Quantiles

$100.0 \%$ maximum 1.0700

$99.5 \%$

$\begin{array}{ll}97.5 \% & 1.0700\end{array}$

$90.0 \% \quad 1.0700$

$75.0 \%$ quartile $\quad 1.0700$

$50.0 \%$ median $\quad 1.0600$

$25.0 \%$ quartile $\quad 1.0250$

$10.0 \% \quad 1.0200$

$2.5 \% \quad 1.0200$

$0.5 \% \quad 1.0200$

$0.0 \%$ minimum 1.0200

Moments

Mean

Std Dev $\quad 0.0234521$

Std Err Mean $\quad 0.0104881$

upper 95\% Mean 1.0791196

lower $95 \%$ Mean 1.0208804

$\mathrm{N}$

Instrument $=\mathrm{M}-14$

Measurement (ppm)

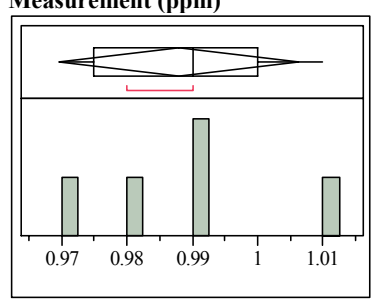

Quantiles

$100.0 \%$ maximum 1.0100

$99.5 \% \quad 1.0100$

$\begin{array}{ll}97.5 \% & 1.0100\end{array}$

$90.0 \% \quad 1.0100$

$75.0 \%$ quartile $\quad 1.0000$

$50.0 \%$ median 0.9900

$25.0 \%$ quartile 0.9750

$10.0 \% \quad 0.9700$

$2.5 \% \quad 0.9700$

$0.5 \% \quad 0.9700$

$0.0 \%$ minimum 0.9700

Moments

Mean $\quad 0.988$

Std Dev $\quad 0.0148324$

Std Err Mean $\quad 0.0066332$

upper $95 \%$ Mean 1.0064169

lower 95\% Mean 0.9695831

$\mathrm{N}$
Exhibit A10f.

Distributions Type $=$ Simulated

Sample Crosscheck Data 5000X

Dilution, Simulant=SME, Known

Concentration $(\mathrm{ppm})=1$,

Anion=Sulfate, ICS-3000

Instrument=M-13

Measurement (ppm)

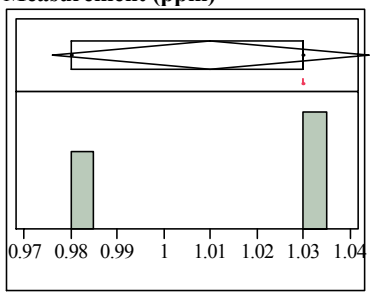

Quantiles

$100.0 \%$ maximum 1.0300

$\begin{array}{ll}99.5 \% & 1.0300 \\ 97.5 \% & 1.0300\end{array}$

$90.0 \% \quad 1.0300$

$75.0 \%$ quartile $\quad 1.0300$

$50.0 \%$

$25.0 \%$ quartile 0.9800

$10.0 \% \quad 0.9800$

$2.5 \% \quad 0.9800$

$0.5 \% \quad 0.9800$

$0.0 \%$ minimum 0.9800

Moments

Mean

0.0273861

Std Err Mean $\quad 0.0122474$

upper $95 \%$ Mean 1.0440044

lower 95\% Mean 0.9759956

$\mathrm{N}$

0 Instrument $=M-14$

Measurement (ppm)

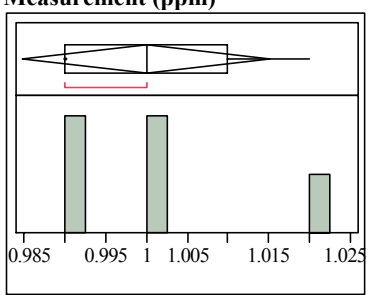

Quantiles

$100.0 \%$ maximum 1.0200

$99.5 \% \quad 1.0200$

$\begin{array}{ll}97.5 \% & 1.0200\end{array}$

$90.0 \% \quad 1.0200$

$75.0 \%$ quartile $\quad 1.0100$

$50.0 \%$ median 1.0000

$25.0 \%$ quartile 0.9900

$10.0 \% \quad 0.9900$

$2.5 \% \quad 0.9900$

$0.5 \% \quad 0.9900$

$0.0 \%$ minimum 0.9900

Moments

Mean

Std Dev $\quad 0.0122474$

Std Err Mean $\quad 0.0054772$

upper $95 \%$ Mean 1.0152072

lower $95 \%$ Mean 0.9847928

$\mathrm{N}$
Exhibit A10g.

Distributions Type=Simulated

Sample Crosscheck Data 5000X

Dilution, Simulant=SME, Known

Concentration $(\mathrm{ppm})=2.5$,

Anion=Nitrate, ICS-3000

Instrument $=M-13$

Measurement (ppm)

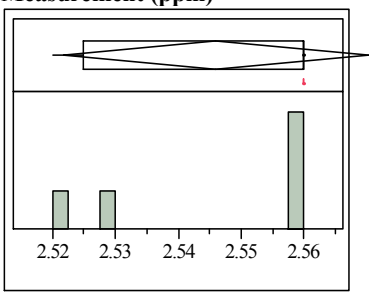

Quantiles

$100.0 \%$ maximum 2.5600

$99.5 \% \quad 2.5600$

$\begin{array}{ll}97.5 \% & 2.5600\end{array}$

$90.0 \% \quad 2.5600$

$75.0 \%$ quartile $\quad 2.5600$

$50.0 \%$ median 2.5600

$25.0 \%$ quartile 2.5250

$10.0 \% \quad 2.5200$

$2.5 \% \quad 2.5200$

$0.5 \% \quad 2.5200$

$0.0 \%$ minimum 2.5200

Moments

Mean $\quad 2.546$

Std Dev $\quad 0.0194936$

Std Err Mean $\quad 0.0087178$

upper $95 \%$ Mean 2.5702045

lower 95\% Mean 2.5217955

$\mathrm{N}$

Instrument $=\mathrm{M}-14$

Measurement (ppm)

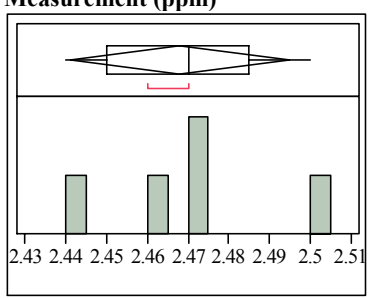

Quantiles

$100.0 \%$ maximum 2.5000

$99.5 \% \quad 2.5000$

$\begin{array}{ll}97.5 \% & 2.5000 \\ 90.0 \% & 2.5000\end{array}$

$90.0 \% \quad 2.5000$

$75.0 \%$ quartile 2.4850

$50.0 \%$ median 2.4700

$25.0 \%$ quartile 2.4500

$10.0 \% \quad 2.4400$

$2.5 \% \quad 2.4400$

$0.5 \% \quad 2.4400$

$0.0 \%$ minimum 2.4400

Moments

Mean

Std Dev $\quad 0.0216795$

Std Err Mean $\quad 0.0096954$

upper $95 \%$ Mean 2.4949186

lower $95 \%$ Mean 2.4410814
Exhibit A10h.

Distributions $\mathrm{Type}=$ Simulated

Sample Crosscheck Data 5000X

Dilution, Simulant=SME, Known

Concentration $(\mathrm{ppm})=6$,

Anion=Formate, ICS-3000

Instrument $=\mathrm{M}-13$

Measurement (ppm)

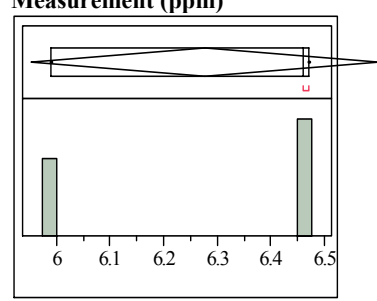

Quantiles

$100.0 \%$ maximum 6.4700

$99.5 \% \quad 6.4700$

$97.5 \% \quad 6.4700$

$90.0 \% \quad 6.4700$

$75.0 \%$ quartile $\quad 6.4700$

$50.0 \%$ median 6.4600

$25.0 \%$ quartile 5.9900

$10.0 \% \quad 5.9900$

$2.5 \% \quad 5.9900$

$0.5 \% \quad 5.9900$

$0.0 \%$ minimum 5.9900

Moments

Std Dev $\quad 0.261113$

Std Err Mean $\quad 0.1167733$

upper $95 \%$ Mean 6.6002146

lower 95\% Mean 5.9517854

Instrument $=\mathrm{M}-14$

Measurement (ppm)

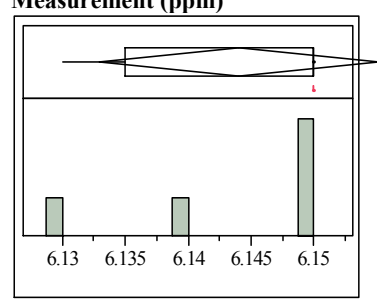

Quantiles

$100.0 \%$ maximum 6.1500

$99.5 \% \quad 6.1500$

$\begin{array}{ll}97.5 \% & 6.1500\end{array}$

$90.0 \% \quad 6.1500$

$75.0 \%$ quartile 6.1500

$50.0 \%$ median 6.1500

$25.0 \%$ quartile $\quad 6.1350$

$10.0 \% \quad 6.1300$

$2.5 \% \quad 6.1300$

$\begin{array}{ll}0.5 \% & 6.1300\end{array}$

$0.0 \%$ minimum 6.1300

Moments

Mean $\quad 6.144$

Std Dev $\quad 0.0089443$

Std Err Mean $\quad 0.004$

upper 95\% Mean 6.1551058

lower 95\% Mean 6.1328942

N 
Exhibit A10i.

Distributions Type $=$ Simulated Sample Crosscheck Data 5000X Dilution, Simulant=SRAT Receipt, Known Concentration (ppm) $=1$, Anion $=$ Chloride, ICS-3000 Instrument $=\mathrm{M}-13$

Measurement (ppm)

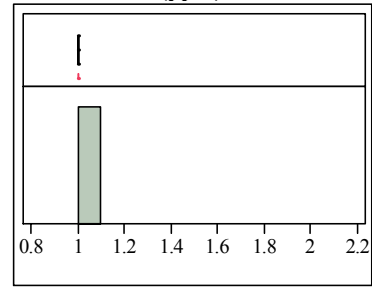

Quantiles

$100.0 \%$ maximum 1.0000

$99.5 \% \quad 1.0000$

$97.5 \% \quad 1.0000$

$90.0 \% \quad 1.0000$

$75.0 \%$ quartile $\quad 1.0000$

$50.0 \%$ median 1.0000

$25.0 \%$ quartile 1.0000

$10.0 \% \quad 1.0000$

$2.5 \% \quad 1.0000$

$0.5 \%-1.0000$

$0.0 \%$ minimum 1.0000

Moments

Mean

Std Dev

Std Err Mean 0

upper $95 \%$ Mean

lower $95 \%$ Mean

$\mathrm{N}$

Instrument=M-14

Measurement (ppm)

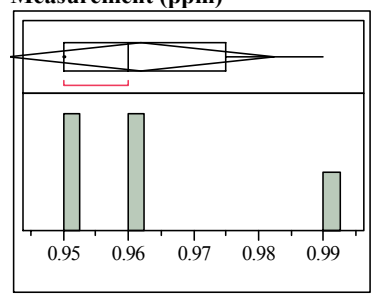

Quantiles

$100.0 \%$ maximum 0.99000

$99.5 \%$

$97.5 \% \quad 0.99000$

$90.0 \% \quad 0.99000$

$75.0 \%$ quartile $\quad 0.97500$

$50.0 \%$ median 0.96000

$25.0 \%$ quartile 0.95000

$10.0 \% \quad 0.95000$

$2.5 \%-0.95000$

$0.5 \% \quad 0.95000$

$0.0 \%$ minimum 0.95000

Moments

Mean

0.962

Std Dev

0.0164317

Std Err Mean $\quad 0.0073485$ upper $95 \%$ Mean 0.9824026 lower $95 \%$ Mean 0.9415974
Exhibit A10j.

Distributions Type $=$ Simulated

Sample Crosscheck Data 5000X

Dilution, Simulant=SRAT Receipt,

Known Concentration $(\mathrm{ppm})=1$,

Anion=Fluoride, ICS-3000

Instrument $=\mathbf{M - 1 3}$

Measurement (ppm)

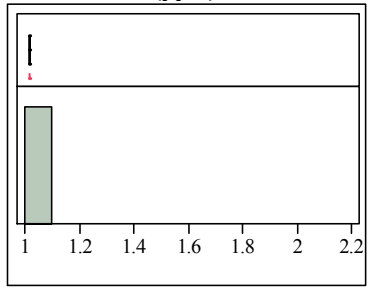

Quantiles

$100.0 \%$ maximum 1.0200

$99.5 \% \quad-1.0200$

$97.5 \% \quad 1.0200$

$90.0 \% \quad 1.0200$

$75.0 \%$ quartile $\quad 1.0200$

$50.0 \%$ median

$25.0 \%$ quartile $\quad 1.0200$

$10.0 \% \quad 1.0200$

$2.5 \% \quad 1.0200$

$0.5 \% \quad 1.0200$

$0.0 \%$ minimum 1.0200

Moments

Mean

Std Dev 1.02

Std Err Mean $\quad 0$

upper $95 \%$ Mean 1.02

lower 95\% Mean 1.02

$\mathrm{N}$

Instrument $=\mathrm{M}-14$

Measurement (ppm)

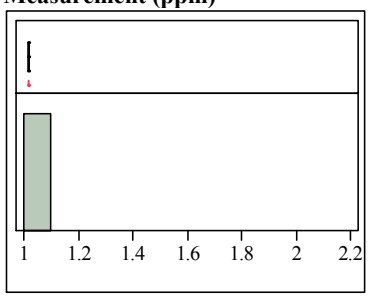

Quantiles

$100.0 \%$ maximum 1.0200

$97.5 \%$

$90.0 \% \quad 1.0200$

$75.0 \%$ quartile $\quad 1.0200$

$50.0 \%$ median 1.0200

$25.0 \%$ quartile 1.0200

$10.0 \% \quad 1.0200$

$2.5 \% \quad 1.0200$

$0.5 \% \quad 1.0200$

$0.0 \%$ minimum 1.0200

Moments

Mean

Std Dev

Std Err Mean

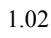

upper $95 \%$ Mean 1.02

lower $95 \%$ Mean 1.02
Exhibit A10k.

Distributions Type $=$ Simulated

Sample Crosscheck Data 5000X

Dilution, Simulant=SRAT Receipt,

Known Concentration $(\mathrm{ppm})=\mathbf{1}$,

Anion $=$ Oxalate, ICS-3000

Instrument $=M-13$

Measurement (ppm)

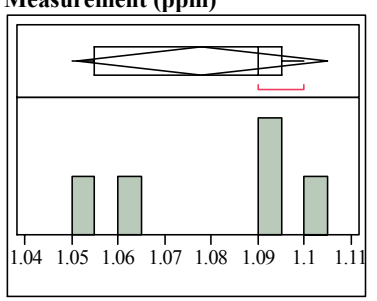

Quantiles

$100.0 \%$ maximum 1.1000

$99.5 \% \quad 1.1000$

$97.5 \% \quad 1.1000$

$90.0 \% \quad 1.1000$

$75.0 \%$ quartile 1.0950

$50.0 \%$ median $\quad 1.0900$

$25.0 \%$ quartile $\quad 1.0550$

$\begin{array}{ll}10.0 \% & 1.0500\end{array}$

$2.5 \% \quad 1.0500$

$0.5 \% \quad 1.0500$

$0.0 \%$ minimum 1.0500

Moments

Mean $\quad 1.078$

Std Dev $\quad 0.0216795$

Std Err Mean $\quad 0.0096954$

upper 95\% Mean 1.1049186

lower 95\% Mean 1.0510814

$\mathrm{N}$

Instrument $=\mathrm{M}-14$

Measurement (ppm)

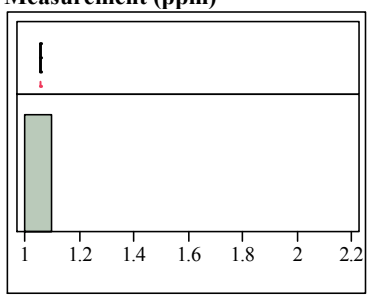

Quantiles

$100.0 \%$ maximum 1.0600

$97.5 \% \quad 1.0600$

$\begin{array}{ll}90.0 \% & 1.0600\end{array}$

$75.0 \%$ quartile $\quad 1.0600$

$50.0 \%$ median 1.0600

$25.0 \%$ quartile 1.0600

$10.0 \% \quad 1.0600$

$2.5 \% \quad 1.0600$

$0.5 \% \quad 1.0600$

$0.0 \%$ minimum 1.0600

Moments

Mean

Std Dev

1.06

Std Err Mean

0
0

upper $95 \%$ Mean 1.06

lower $95 \%$ Mean 1.06

$\mathrm{N}$
Exhibit A101.

Distributions Type $=$ Simulated Sample Crosscheck Data 5000X

Dilution, Simulant=SRAT Receipt,

Known Concentration $(\mathrm{ppm})=1$,

Anion $=$ Phosphate, ICS-3000

Instrument=M-13

Measurement (ppm)

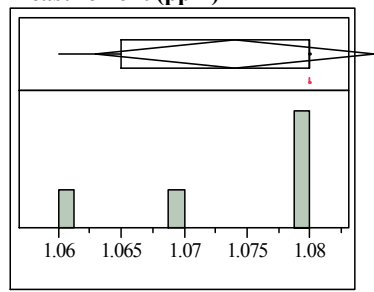

Quantiles

$100.0 \%$ maximum 1.0800

$99.5 \% \quad 1.0800$

$97.5 \%$

$90.0 \% \quad 1.0800$

$75.0 \%$ quartile $\quad 1.0800$

$50.0 \%$ median 1.0800

$25.0 \%$ quartile $\quad 1.0650$

$10.0 \% \quad 1.0600$

$2.5 \% \quad 1.0600$

$0.5 \% \quad 1.0600$

$0.0 \%$ minimum 1.0600

Moments

Mean

Std Err Mean $\quad 0.0089443$

upper 95\% Mean 1.0851058

lower 95\% Mean 1.0628942

$\mathrm{N}$

Instrument $=\mathrm{M}-14$

Measurement (ppm)

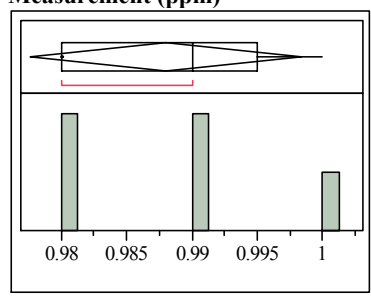

Quantiles

$100.0 \%$ maximum 1.0000

$99.5 \% \quad 1.0000$

$97.5 \% \quad 1.0000$

$90.0 \% \quad 1.0000$

$75.0 \%$ quartile $\quad 0.9950$

$50.0 \%$ median 0.9900

$25.0 \%$ quartile 0.9800

$10.0 \% \quad 0.9800$

$2.5 \% \quad 0.9800$

$0.5 \% \quad 0.9800$

$0.0 \%$ minimum 0.9800

Moments

Mean 0.988

Std Dev $\quad 0.0083666$

Std Err Mean 0.0037417

upper $95 \%$ Mean 0.9983885

lower 95\% Mean 0.9776115

$\mathrm{N}$ 
Exhibit A10m.

Distributions Type $=$ Simulated

Sample Crosscheck Data 5000X

Dilution, Simulant=SRAT Receipt,

Known Concentration $(\mathbf{p p m})=1$,

Anion=Sulfate, ICS-3000

Instrument $=\mathrm{M}-13$

Measurement (ppm)

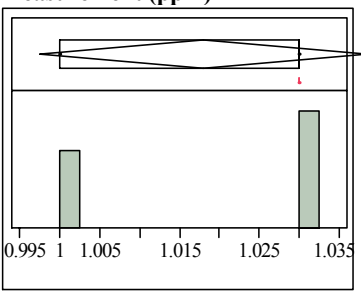

Quantiles

$100.0 \%$ maximum 1.0300

$99.5 \% \quad 1.0300$

$97.5 \% \quad 1.0300$

$90.0 \% \quad 1.0300$

$75.0 \%$ quartile $\quad 1.0300$

$50.0 \%$ median 1.0300

$25.0 \%$ quartile $\quad 1.0000$

$10.0 \% \quad 1.0000$

$2.5 \% \quad 1.0000$

$0.5 \% \quad 1.0000$

$0.0 \%$ minimum 1.0000

Moments

Mean $\quad 1.018$

Std Dev $\quad 0.0164317$

Std Err Mean $\quad 0.0073485$

upper 95\% Mean 1.0384026

lower 95\% Mean 0.9975974

$\mathrm{N}$

Instrument $=\mathrm{M}-14$

Measurement (ppm)

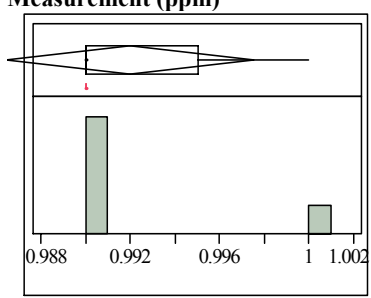

Quantiles

$100.0 \%$ maximum 1.0000

$99.5 \% \quad 1.0000$

$\begin{array}{ll}97.5 \% & 1.0000\end{array}$

$90.0 \% \quad 1.0000$

$75.0 \%$ quartile $\quad 0.9950$

$50.0 \%$ median 0.9900

$25.0 \%$ quartile 0.9900

$10.0 \%-0.9900$

$2.5 \% \quad 0.9900$

$0.5 \% \quad 0.9900$

$0.0 \%$ minimum 0.9900

Moments

Mean $\quad 0.992$

Std Dev $\quad 0.0044721$

Std Err Mean $\quad 0.002$

upper $95 \%$ Mean 0.9975529

lower 95\% Mean 0.9864471

$\mathrm{N}$
Exhibit A10n.

Distributions $T y p e=$ Simulated

Sample Crosscheck Data 5000X

Dilution, Simulant=SRAT Receipt,

Known Concentration $(\mathrm{ppm})=1.5$,

Anion=Formate, ICS-3000

Instrument=M-13

Measurement (ppm)

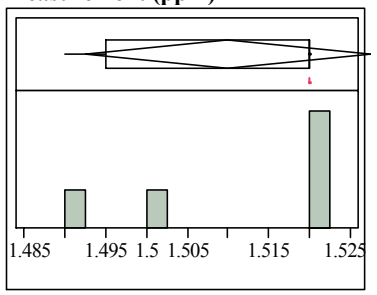

Quantiles

$100.0 \%$ maximum 1.5200

$\begin{array}{ll}9.5 \% & 1.5200 \\ 97.5 \% & 1.5200\end{array}$

$90.0 \% \quad 1.5200$

$75.0 \%$ quartile $\quad 1.5200$

$50.0 \%$ median 1.5200

$25.0 \%$ quartile 1.4950

$10.0 \% \quad 1.4900$

$\begin{array}{ll}10.0 \% & 1.4900\end{array}$

$0.5 \% \quad 1.4900$

$0.0 \%$ minimum 1.4900

Moments

Mean

0.014142

Std Err Mean $\quad 0.0063246$

upper $95 \%$ Mean 1.5275598

lower 95\% Mean 1.4924402

$\mathrm{N}$

Instrument=M-14

Measurement (ppm)

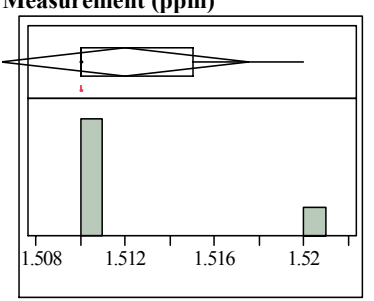

Quantiles

$100.0 \%$ maximum 1.5200

$99.5 \% \quad 1.5200$

$\begin{array}{ll}97.5 \% & 1.5200\end{array}$

$90.0 \% \quad 1.5200$

$75.0 \%$ quartile $\quad 1.5150$

$50.0 \%$ median 1.5100

$25.0 \%$ quartile $\quad 1.5100$

$10.0 \% \quad 1.5100$

$2.5 \% \quad 1.5100$

$0.5 \% \quad 1.5100$

$0.0 \%$ minimum 1.5100

Moments

Mean $\quad 1.512$

Std Dev $\quad 0.0044721$

Std Err Mean $\quad 0.002$

upper $95 \%$ Mean 1.5175529

lower 95\% Mean 1.5064471
Exhibit A10o.

Distributions Type $=$ Simulated

Sample Crosscheck Data 5000X

Dilution, Simulant=SRAT Receipt,

Known Concentration $(\mathrm{ppm})=1.5$,

Anion=Nitrate, ICS-3000

Instrument $=M-13$

Measurement (ppm)

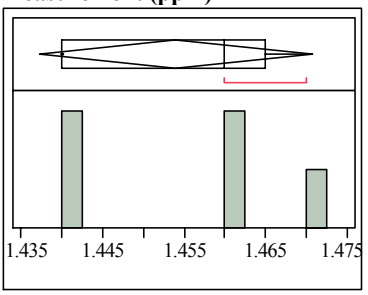

Quantiles

$100.0 \%$ maximum 1.4700

$99.5 \% \quad 1.4700$

$97.5 \% \quad 1.4700$

$90.0 \% \quad 1.4700$

$75.0 \%$ quartile $\quad 1.4650$

$50.0 \%$ median 1.4600

$25.0 \%$ quartile 1.4400

$10.0 \% \quad 1.4400$

$2.5 \% \quad 1.4400$

$0.5 \% \quad 1.4400$

$0.0 \%$ minimum 1.4400

Moments

Mean

$\quad 0.0134164$

Std Err Mean $\quad 0.006$

upper $95 \%$ Mean 1.4706587

lower $95 \%$ Mean 1.4373413

Instrument=M-14

Measurement (ppm)

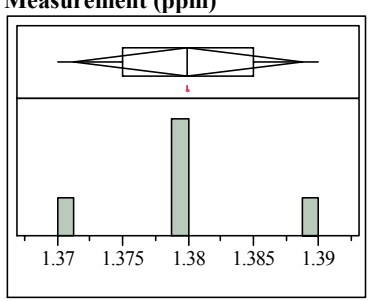

Quantiles

$100.0 \%$ maximum 1.3900

$99.5 \% \quad 1.3900$

$\begin{array}{ll}97.5 \% & 1.3900\end{array}$

$90.0 \% \quad 1.3900$

$75.0 \%$ quartile $\quad 1.3850$

$50.0 \%$ median 1.3800

$25.0 \%$ quartile $\quad 1.3750$

$10.0 \% \quad 1.3700$

$\begin{array}{ll}2.5 \% & 1.3700\end{array}$

$\begin{array}{ll}0.5 \% & 1.3700\end{array}$

$0.0 \%$ minimum 1.3700

Moments

Mean

0.007071

Std Err Mean $\quad 0.0031623$

upper $95 \%$ Mean 1.3887799

lower $95 \%$ Mean 1.3712201
Exhibit A10p.

Distributions Type $=$ Simulated

Sample Crosscheck Data 5000X

Dilution, Simulant=SRAT Receipt,

Known Concentration $(\mathrm{ppm})=1.5$,

Anion=Nitrite, ICS-3000

Instrument $=\mathrm{M}-13$

Measurement (ppm)

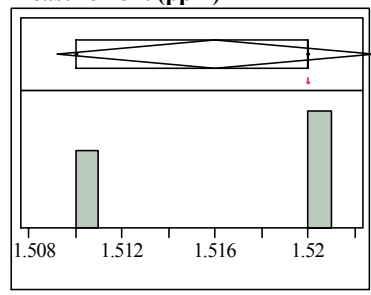

Quantiles

$100.0 \%$ maximum 1.5200

$99.5 \% \quad 1.5200$

$97.5 \% \quad 1.5200$

$90.0 \% \quad 1.5200$

$75.0 \%$ quartile $\quad 1.5200$

$50.0 \%$ median $\quad 1.5200$

$25.0 \%$ quartile 1.5100

$10.0 \% \quad 1.5100$

$2.5 \% \quad 1.5100$

$0.5 \% \quad 1.5100$

$0.0 \%$ minimum 1.5100

Moments

Std Dev $\quad 0.0054772$

Std Err Mean $\quad 0.0024495$

upper 95\% Mean 1.5228009

lower 95\% Mean 1.5091991

Instrument $=\mathrm{M}-14$

Measurement (ppm)

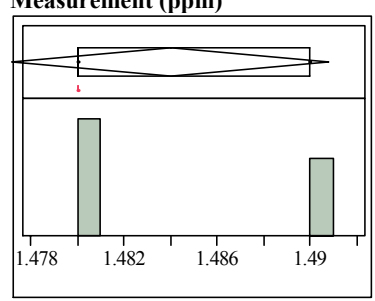

Quantiles

$100.0 \%$ maximum 1.4900

$99.5 \% \quad 1.4900$

$\begin{array}{ll}97.5 \% & 1.4900\end{array}$

$90.0 \% \quad 1.4900$

$75.0 \%$ quartile $\quad 1.4900$

$50.0 \%$ median 1.4800

$25.0 \%$ quartile $\quad 1.4800$

$10.0 \% \quad 1.4800$

$2.5 \% \quad 1.4800$

$\begin{array}{ll}0.5 \% & 1.4800\end{array}$

$0.0 \%$ minimum 1.4800

Moments

Mean $\quad 1.484$

Std Dev $\quad 0.0054772$

Std Err Mean $\quad 0.0024495$

upper 95\% Mean 1.4908009

lower 95\% Mean 1.4771991

N 
Exhibit A10q.

Distributions Type $=$ Simulated

Sample Crosscheck Data 500X

Dilution, Simulant=SME, Known

Concentration $(\mathrm{ppm})=1$,

Anion $=$ Chloride, ICS-3000

Instrument $=\mathrm{M}-13$

Measurement (ppm)

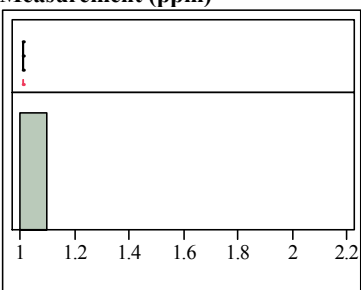

Quantiles

$100.0 \%$ maximum 1.0100

$99.5 \% \quad 1.0100$

$97.5 \% \quad 1.0100$

$90.0 \% \quad 1.0100$

$75.0 \%$ quartile $\quad 1.0100$

$50.0 \%$ median 1.0100

$25.0 \%$ quartile $\quad 1.0100$

$10.0 \% \quad 1.0100$

$2.5 \% \quad 1.0100$

$0.5 \% \quad 1.0100$

$0.0 \%$ minimum 1.0100

Moments

Mean $\quad 1.01$

Std Dev

Std Err Mean 0

upper 95\% Mean 1.01

lower $95 \%$ Mean 1.01

$\mathrm{N}$

Instrument $=\mathrm{M}-14$

Measurement (ppm)

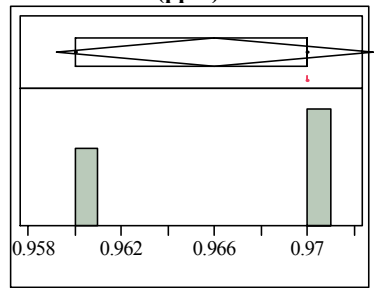

Quantiles

$100.0 \%$ maximum 0.97000

$99.5 \% \quad 0.97000$

$97.5 \% \quad 0.97000$

$90.0 \% \quad 0.97000$

$75.0 \%$ quartile 0.97000

$50.0 \%$ median 0.97000

$25.0 \%$ quartile 0.96000

$10.0 \% \quad 0.96000$

$2.5 \% \quad 0.96000$

$0.5 \% \quad 0.96000$

$0.0 \%$ minimum 0.96000

Moments

Mean $\quad 0.966$

Std Dev $\quad 0.0054772$

Std Err Mean $\quad 0.0024495$

upper 95\% Mean 0.9728009

lower $95 \%$ Mean 0.9591991

$\mathrm{N}$
Exhibit A10r.

Distributions Type $=$ Simulated

Sample Crosscheck Data 500X

Dilution, Simulant=SME, Known

Concentration $(\mathrm{ppm})=1$,

Anion=Fluoride, ICS-3000

Instrument $=M-13$

Measurement (ppm)

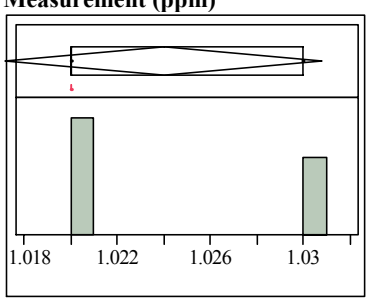

Quantiles

$100.0 \%$ maximum 1.0300

$99.5 \% \quad 1.0300$

$97.5 \% \quad 1.0300$

$90.0 \% \quad 1.0300$

$75.0 \%$ quartile $\quad 1.0300$

$50.0 \%$ median 1.0200

$25.0 \%$ quartile $\quad 1.0200$

$10.0 \% \quad 1.0200$

$2.5 \% \quad 1.0200$

$0.5 \% \quad 1.0200$

$0.0 \%$ minimum 1.0200

Moments

Mean $\quad 1.024$

Std Dev $\quad 0.0054772$

Std Err Mean $\quad 0.0024495$

upper 95\% Mean 1.0308009

lower 95\% Mean 1.0171991

$\mathrm{N}$

Instrument $=$ M-14

Measurement (ppm)

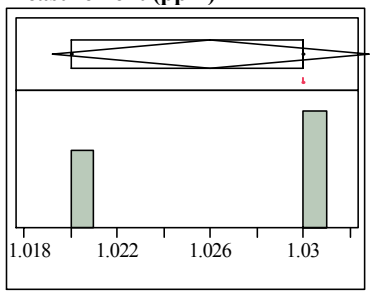

Quantiles

$100.0 \%$ maximum 1.0300

$99.5 \% \quad 1.0300$

$97.5 \% \quad 1.0300$

$90.0 \% \quad 1.0300$

$75.0 \%$ quartile $\quad 1.0300$

$50.0 \%$ median 1.0300

$25.0 \%$ quartile $\quad 1.0200$

$10.0 \% \quad 1.0200$

$\begin{array}{ll}2.5 \% & 1.0200\end{array}$

$0.5 \% \quad 1.0200$

$0.0 \%$ minimum 1.0200

Moments

Mean 1.026

Std Dev $\quad 0.0054772$

Std Err Mean $\quad 0.0024495$

upper 95\% Mean 1.0328009

lower 95\% Mean 1.0191991

$\mathrm{N}$
Exhibit A10s.

Distributions $\mathrm{Type}=$ Simulated

Sample Crosscheck Data 500X

Dilution, Simulant=SME, Known

Concentration $(\mathrm{ppm})=1$,

Anion $=$ Nitrite, ICS-3000

Instrument $=$ M-13

Measurement (ppm)

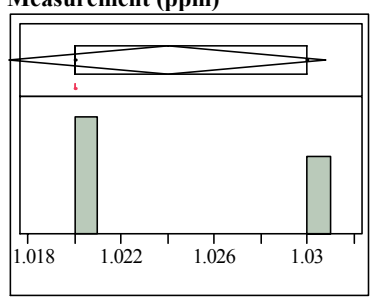

Quantiles

$100.0 \%$ maximum 1.0300

$99.5 \% \quad 1.0300$

$97.5 \% \quad 1.0300$

$90.0 \% \quad 1.0300$

$75.0 \%$ quartile 1.0300

$50.0 \%$ median 1.0200

$25.0 \%$ quartile $\quad 1.0200$

$\begin{array}{ll}10.0 \% & 1.0200\end{array}$

$2.5 \% \quad 1.0200$

$0.5 \% \quad 1.0200$

$0.0 \%$ minimum 1.0200

Moments

Mean 1.024

Std Dev $\quad 0.0054772$

Std Err Mean $\quad 0.0024495$

upper 95\% Mean 1.0308009

lower 95\% Mean 1.0171991

$\mathrm{N}$

Instrument $=\mathrm{M}-14$

Measurement (ppm)

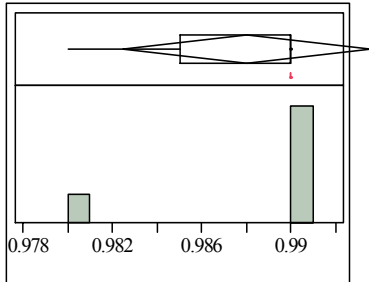

Quantiles

$100.0 \%$ maximum 0.99000

$99.5 \% \quad 0.99000$

$97.5 \% \quad 0.99000$

$90.0 \% \quad 0.99000$

$75.0 \%$ quartile 0.99000

$50.0 \%$ median 0.99000

$10.0 \% \quad 0.98000$

$2.5 \% \quad 0.98000$

$0.5 \% \quad 0.98000$

$0.0 \%$ minimum 0.98000

Moments

Mean

Std Dev $\quad 0.0044721$

Std Err Mean $\quad 0.002$

upper 95\% Mean 0.9935529

lower $95 \%$ Mean 0.9824471

$\mathrm{N}$
Exhibit A10t.

Distributions Type $=$ Simulated

Sample Crosscheck Data 500X

Dilution, Simulant=SME, Known

Concentration $(\mathrm{ppm})=\mathbf{2 . 5}$,

Anion $=$ Oxalate, ICS-3000

Instrument=M-13

Measurement (ppm)

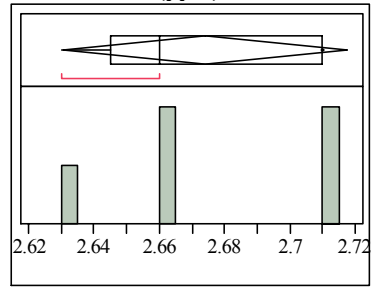

Quantiles

$100.0 \%$ maximum 2.7100

$99.5 \% \quad 2.7100$

$97.5 \% \quad 2.7100$

$90.0 \% \quad 2.7100$

$75.0 \%$ quartile 2.7100

$50.0 \%$ median 2.6600

$25.0 \%$ quartile 2.6450

$10.0 \% \quad 2.6300$

$2.5 \% \quad 2.6300$

$0.5 \% \quad 2.6300$

$0.0 \%$ minimum 2.6300

Moments

Mean $\quad 2674$

Std Dev $\quad 0.0350714$

Std Err Mean $\quad 0.0156844$

upper 95\% Mean 2.7175468

lower 95\% Mean 2.6304532

$\mathrm{N}$

Instrument $=\mathrm{M}-14$

Measurement (ppm)

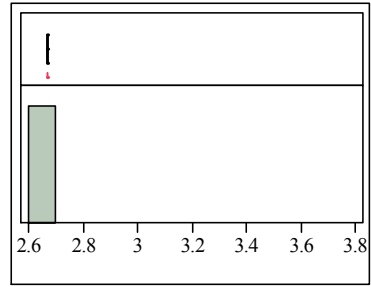

Quantiles

$100.0 \%$ maximum 2.6700

$99.5 \% \quad 2.6700$

$97.5 \% \quad 2.6700$

$90.0 \% \quad 2.6700$

$75.0 \%$ quartile 2.6700

$50.0 \%$ median 2.6700

$25.0 \%$ quartile 2.6700

$10.0 \% \quad 2.6700$

$\begin{array}{ll}2.5 \% & 2.6700\end{array}$

$0.5 \% \quad 2.6700$

$0.0 \%$ minimum 2.6700

Moments

Mean 2.67

Std Dev

upper 95\% Mean 2.67

lower 95\% Mean 2.67

$\mathrm{N}$ 
Exhibit A10u.

Distributions Type $=$ Simulated

Sample Crosscheck Data 500X

Dilution, Simulant=SME, Known

Concentration $(\mathrm{ppm})=\mathbf{2 . 5}$,

Anion=Phosphate, ICS-3000

Instrument $=\mathrm{M}-13$

Measurement (ppm)

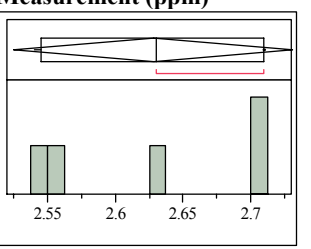

Quantiles

$100.0 \%$ maximum 2.7100

$99.5 \% \quad 2.7100$

$97.5 \% \quad 2.7100$

$90.0 \% \quad 2.7100$

$75.0 \%$ quartile 2.7100

$50.0 \%$ median 2.6300

$25.0 \%$ quartile 2.5450

$10.0 \% \quad 2.5400$

$2.5 \% \quad 2.5400$

$0.5 \% \quad 2.5400$

$0.0 \%$ minimum 2.5400

Moments

Mean 2628

Std Dev $\quad 0.0825833$

Std Err Mean $\quad 0.0369324$

upper 95\% Mean 2.7305407

lower 95\% Mean 2.5254593

$\mathrm{N}$

Instrument $=\mathrm{M}-14$

Measurement (ppm)

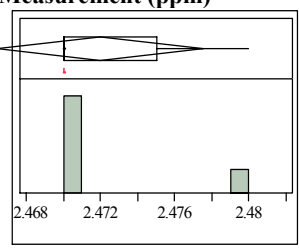

Quantiles

$100.0 \%$ maximum 2.4800

$99.5 \% \quad 2.4800$

$97.5 \% \quad 2.4800$

$90.0 \% \quad 2.4800$

$75.0 \%$ quartile 2.4750

$50.0 \%$ median 2.4700

$25.0 \%$ quartile 2.4700

$10.0 \% \quad 2.4700$

$2.5 \% \quad 2.4700$

$0.5 \% \quad 2.4700$

$0.0 \%$ minimum 2.4700

Moments

Mean 2.472

Std Dev $\quad 0.0044721$

Std Err Mean $\quad 0.002$

upper $95 \%$ Mean 2.4775529

lower 95\% Mean 2.4664471
Exhibit A10v.

Distributions $\mathbf{T y p e}=$ Simulated

Sample Crosscheck Data 500X

Dilution, Simulant=SME, Known

Concentration $(\mathrm{ppm})=\mathbf{2 . 5}$,

Anion $=$ Sulfate, ICS-3000

Instrument=M-13

Measurement (ppm)

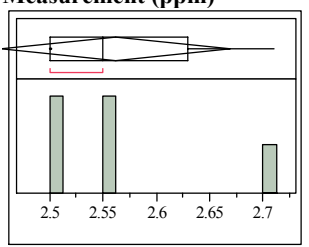

Quantiles

$100.0 \%$ maximum 2.7100

$99.5 \% \quad 2.7100$

$97.5 \% \quad 2.7100$

$90.0 \% \quad 2.7100$

$75.0 \%$ quartile 2.6300

$50.0 \%$ median 2.5500

$25.0 \%$ quartile 2.5000

$10.0 \% \quad 2.5000$

$2.5 \% \quad 2.5000$

$0.5 \% \quad 2.5000$

$0.0 \%$ minimum 2.5000

Moments

Mean $\quad 2.562$

Std Dev $\quad 0.0864292$

Std Err Mean $\quad 0.0386523$

upper 95\% Mean 2.669316

lower 95\% Mean 2.454684

$\mathrm{N}$

Instrument $=\mathrm{M}-14$

Measurement (ppm)

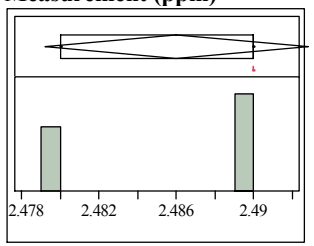

Quantiles

$100.0 \%$ maximum 2.4900

$99.5 \% \quad 2.4900$

$97.5 \% \quad 2.4900$

$90.0 \% \quad 2.4900$

$75.0 \%$ quartile 2.4900

$50.0 \%$ median 2.4900

$25.0 \%$ quartile 2.4800

$10.0 \% \quad 2.4800$

$2.5 \% \quad 2.4800$

$0.5 \% \quad 2.4800$

$0.0 \%$ minimum 2.4800

Moments

Mean 2.486

Std Dev $\quad 0.0054772$

Std Err Mean $\quad 0.0024495$

upper 95\% Mean 2.4928009

lower 95\% Mean 2.4791991

$\mathrm{N}$
Exhibit A10w.

Distributions Type $=$ Simulated

Sample Crosscheck Data 500X

Dilution, Simulant=SME, Known

Concentration $(\mathrm{ppm})=25$,

Anion=Nitrate, ICS-3000

Instrument $=\mathrm{M}-13$

Measurement (ppm)

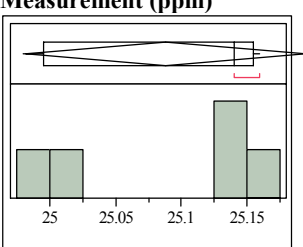

Quantiles

$100.0 \%$ maximum 25.160

$99.5 \% \quad 25.160$

$97.5 \% \quad 25.160$

$90.0 \% \quad 25.160$

$75.0 \%$ quartile 25.155

$50.0 \%$ median $\quad 25.140$

$25.0 \%$ quartile 24.995

$10.0 \% \quad 24.980$

$2.5 \% \quad 24.980$

$0.5 \% \quad 24.980$

$0.0 \%$ minimum 24.980

Moments

Mean $\quad 25.088$

Std Dev $\quad 0.0858487$

Std Err Mean $\quad 0.0383927$

upper 95\% Mean 25.194595

lower 95\% Mean 24.981405

$\mathrm{N}$

Instrument $=\mathrm{M}-14$

Measurement (ppm)

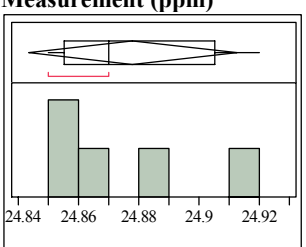

Quantiles

$100.0 \%$ maximum 24.920

$99.5 \% \quad 24.920$

$97.5 \% \quad 24.920$

$90.0 \% \quad 24.920$

$75.0 \%$ quartile 24.905

$50.0 \%$ median 24.870

$25.0 \%$ quartile $\quad 24.855$

$10.0 \% \quad 24.850$

$\begin{array}{ll}2.5 \% & 24.850\end{array}$

$0.5 \% \quad 24.850$

$0.0 \%$ minimum 24.850

Moments

Mean 24.878

Std Dev $\quad 0.0277489$

Std Err Mean $\quad 0.0124097$

upper 95\% Mean 24.912455

lower $95 \%$ Mean 24.843545

$\mathrm{N}$
Exhibit A10x.

Distributions Type $=$ Simulated

Sample Crosscheck Data 500X

Dilution, Simulant=SME, Known

Concentration $(\mathrm{ppm})=60$,

Anion=Formate, ICS-3000

Instrument $=\mathrm{M}-13$

Measurement (ppm)

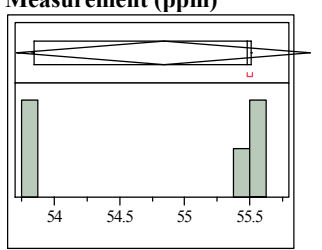

Quantiles

$100.0 \%$ maximum 55.520

$99.5 \% \quad 55.520$

$97.5 \% \quad 55.520$

$90.0 \% \quad 55.520$

$75.0 \%$ quartile $\quad 55.510$

$50.0 \%$ median 55.480

$25.0 \%$ quartile 53.845

$10.0 \% \quad 53.830$

$2.5 \% \quad 53.830$

$0.5 \% \quad 53.830$

$0.0 \%$ minimum 53.830

Moments

Mean

$\begin{array}{ll}0.9066532 \\ \text { Std Err Mean } & 0.4054676\end{array}$

upper $95 \%$ Mean 55.963759

lower 95\% Mean 53.712241

$\mathrm{N}$

Instrument $=\mathrm{M}-14$

Measurement (ppm)

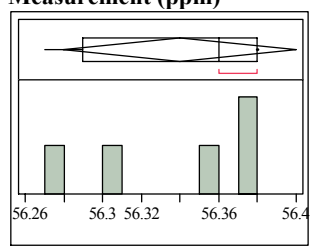

Quantiles

$100.0 \%$ maximum 56.380

$99.5 \% \quad 56.380$

$97.5 \% \quad 56.380$

$90.0 \% \quad 56.380$

$75.0 \%$ quartile 56.380

$50.0 \%$ median 56.360

$25.0 \%$ quartile $\quad 56.290$

$10.0 \% \quad 56.270$

$2.5 \% \quad 56.270$

$0.5 \% \quad 56.270$

$0.0 \%$ minimum 56.270

Moments

Mean $\quad 56.34$

Std Dev $\quad 0.0484768$

Std Err Mean $\quad 0.0216795$

upper 95\% Mean 56.400192

lower $95 \%$ Mean 56.279808

$\mathrm{N}$ 
Exhibit A10x.

Distributions Type $=$ Simulated Sample Crosscheck Data 500X Dilution, Simulant=SRAT Receipt, Known Concentration $(\mathbf{p p m})=1$, Anion $=$ Chloride, ICS-3000

Instrument=M-13

Measurement (ppm)

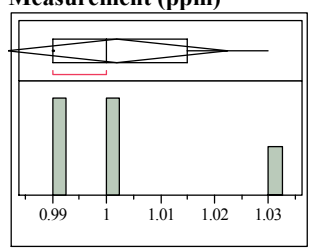

Quantiles

$100.0 \%$ maximum 1.0300

$99.5 \% \quad 1.0300$

$97.5 \% \quad 1.0300$

$90.0 \% \quad 1.0300$

$75.0 \%$ quartile $\quad 1.0150$

$50.0 \%$ median 1.0000

$25.0 \%$ quartile 0.9900

$10.0 \% \quad 0.9900$

$2.5 \% \quad 0.9900$

$0.5 \% \quad 0.9900$

$0.0 \%$ minimum 0.9900

Moments

Mean 1.002

Std Dev $\quad 0.0164317$

Std Err Mean $\quad 0.0073485$

upper $95 \%$ Mean 1.0224026

lower 95\% Mean 0.9815974

$\mathrm{N}$

Instrument $=\mathrm{M}-14$

Measurement (ppm)

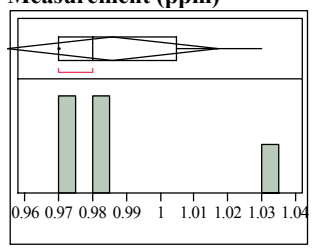

Quantiles

$100.0 \%$ maximum 1.0300

$99.5 \% \quad 1.0300$

$97.5 \% \quad 1.0300$

$90.0 \% \quad 1.0300$

$75.0 \%$ quartile $\quad 1.0050$

$50.0 \%$ median $\quad 0.9800$

$25.0 \%$ quartile 0.9700

$10.0 \% \quad 0.9700$

$2.5 \% \quad 0.9700$

$0.5 \% \quad 0.9700$

$0.0 \%$ minimum 0.9700

Moments

Mean $\quad 0.986$

Std Dev $\quad 0.0250998$

Std Err Mean $\quad 0.011225$

upper 95\% Mean 1.0171655

lower $95 \%$ Mean 0.9548345

$\mathrm{N}$
Exhibit A10y.

Distributions $\mathrm{Type}=$ Simulated

Sample Crosscheck Data 500X

Dilution, Simulant=SRAT Receipt,

Known Concentration $(\mathrm{ppm})=1$,

Anion=Fluoride, ICS-3000

Instrument $=\mathrm{M}-13$

Measurement (ppm)

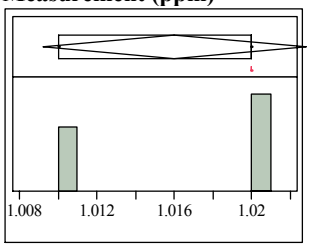

Quantiles

$100.0 \%$ maximum 1.0200

$99.5 \% \quad 1.0200$

$97.5 \% \quad 1.0200$

$90.0 \% \quad 1.0200$

$75.0 \%$ quartile $\quad 1.0200$

$50.0 \%$ median 1.0200

$25.0 \%$ quartile $\quad 1.0100$

$10.0 \% \quad 1.0100$

$2.5 \% \quad 1.0100$

$0.5 \% \quad 1.0100$

$0.0 \%$ minimum 1.0100

Moments

Mean $\quad 1.016$

Std Dev $\quad 0.0054772$

Std Err Mean $\quad 0.0024495$

upper $95 \%$ Mean 1.0228009

lower 95\% Mean 1.0091991

$\mathrm{N}$

Instrument $=\mathbf{M}-14$

Measurement (ppm)

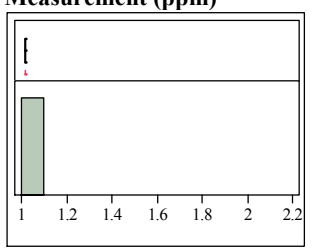

Quantiles

$100.0 \%$ maximum 1.0200

$99.5 \% \quad 1.0200$

$97.5 \% \quad 1.0200$

$90.0 \% \quad 1.0200$

$75.0 \%$ quartile $\quad 1.0200$

$50.0 \%$ median 1.0200

$25.0 \%$ quartile $\quad 1.0200$

$10.0 \% \quad 1.0200$

$\begin{array}{ll}2.5 \% & 1.0200\end{array}$

$0.5 \% \quad 1.0200$

$0.0 \%$ minimum 1.0200

Moments

Mean

Std Dev

upper 95\% Mean 1.02

lower 95\% Mean 1.02

$\mathrm{N}$
Exhibit A10z.

Distributions Type $=$ Simulated

Sample Crosscheck Data 500X

Dilution, Simulant=SRAT Receipt,

Known Concentration $(\mathrm{ppm})=2.5$,

Anion=Oxalate, ICS-3000

Instrument $=\mathrm{M}-\mathbf{1 3}$

Measurement (ppm)

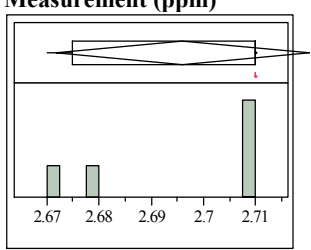

Quantiles

$100.0 \%$ maximum 2.7100

$99.5 \% \quad 2.7100$

$97.5 \% \quad 2.7100$

$90.0 \% \quad 2.7100$

$75.0 \%$ quartile 2.7100

$50.0 \%$ median $\quad 2.7100$

$25.0 \%$ quartile 2.6750

$10.0 \% \quad 2.6700$

$2.5 \% \quad 2.6700$

$0.5 \% \quad 2.6700$

$0.0 \%$ minimum 2.6700

Moments

Mean $\quad 2.696$

Std Dev $\quad 0.0194936$

Std Err Mean $\quad 0.0087178$

upper 95\% Mean 2.7202045

lower 95\% Mean 2.6717955

$\mathrm{N}$
Instrument $=$ M-14

Measurement (ppm)

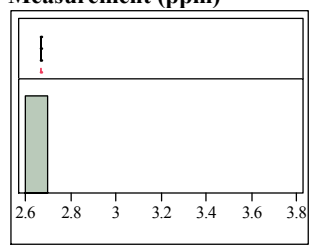

Quantiles

$100.0 \%$ maximum 2.6700

$99.5 \% \quad 2.6700$

$97.5 \% \quad 2.6700$

$90.0 \% \quad 2.6700$

$75.0 \%$ quartile 2.6700

$50.0 \%$ median 2.6700

$25.0 \%$ quartile $\quad 2.6700$

$\begin{array}{ll}10.0 \% & 2.6700\end{array}$

$\begin{array}{ll}2.5 \% & 2.6700\end{array}$

$0.5 \% \quad 2.6700$

$0.0 \%$ minimum 2.6700

Moments

Mean

Std Dev

2.67

Std Err Mea

0
0

upper $95 \%$ Mea

lower $95 \%$ Mean 2.67

$\mathrm{N}$
Exhibit A10aa.

Distributions Type $=$ Simulated Sample Crosscheck Data 500X

Dilution, Simulant=SRAT Receipt,

Known Concentration $(\mathrm{ppm})=2.5$,

Anion $=$ Phosphate, ICS-3000

Instrument $=\mathrm{M}-13$

Measurement (ppm)

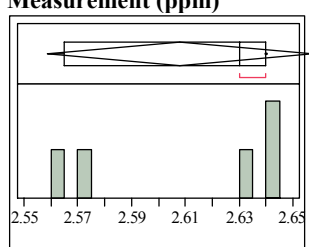

Quantiles

$100.0 \%$ maximum 2.6400

$99.5 \% \quad 2.6400$

$97.5 \% \quad 2.6400$

$90.0 \% \quad 2.6400$

$75.0 \%$ quartile 2.6400

$50.0 \%$ median 2.6300

$25.0 \%$ quartile $\quad 2.5650$

$10.0 \% \quad 2.5600$

$2.5 \% \quad 2.5600$

$0.5 \% \quad 2.5600$

$0.0 \%$ minimum 2.5600

Moments

Mean 2.608

Std Dev $\quad 0.0396232$

Std Err Mean $\quad 0.01772$

upper 95\% Mean 2.6571987

lower 95\% Mean 2.5588013

$\mathrm{N}$

Instrument $=\mathrm{M}-14$

Measurement (ppm)

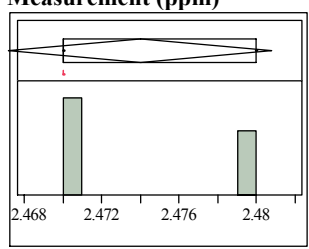

Quantiles

$100.0 \%$ maximum 2.4800

$99.5 \% \quad 2.4800$

$97.5 \% \quad 2.4800$

$90.0 \% \quad 2.4800$

$75.0 \%$ quartile 2.4800

$50.0 \%$ median 2.4700

$25.0 \%$ quartile 2.4700

$10.0 \% \quad 2.4700$

$2.5 \% \quad 2.4700$

$0.5 \% \quad 2.4700$

$0.0 \%$ minimum 2.4700

Moments

Mean 2.474

Std Dev $\quad 0.0054772$

Std Err Mean 0.0024495

upper 95\% Mean 2.4808009

lower 95\% Mean 2.4671991

$\mathrm{N}$ 
Exhibit A10ab.

Distributions Type $=$ Simulated

Sample Crosscheck Data 500X

Dilution, Simulant=SRAT Receipt,

Known Concentration $(\mathrm{ppm})=\mathbf{2 . 5}$,

Anion=Sulfate, ICS-3000

Instrument $=\mathrm{M}-13$

Measurement (ppm)

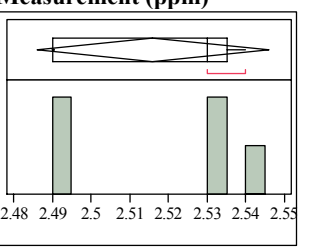

Quantiles

$100.0 \%$ maximum 2.5400

$99.5 \% \quad 2.5400$

$97.5 \% \quad 2.5400$

$90.0 \% \quad 2.5400$

$75.0 \%$ quartile 2.5350

$50.0 \%$ median 2.5300

$25.0 \%$ quartile 2.4900

$10.0 \% \quad 2.4900$

$2.5 \% \quad 2.4900$

$0.5 \% \quad 2.4900$

$0.0 \%$ minimum 2.4900

Moments

Mean 2.516

Std Dev $\quad 0.0240832$

Std Err Mean $\quad 0.0107703$

upper $95 \%$ Mean 2.5459032

lower 95\% Mean 2.4860968

$\mathrm{N}$

Instrument $=\mathrm{M}-14$

Measurement (ppm)

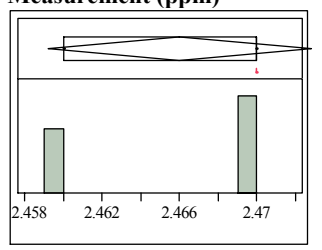

Quantiles

$100.0 \%$ maximum 2.4700

$99.5 \% \quad 2.4700$

$97.5 \% \quad 2.4700$

$90.0 \% \quad 2.4700$

$75.0 \%$ quartile 2.4700

$50.0 \%$ median 2.4700

$25.0 \%$ quartile 2.4600

$\begin{array}{ll}10.0 \% & 2.4600\end{array}$

$2.5 \% \quad 2.4600$

$0.5 \% \quad 2.4600$

$0.0 \%$ minimum 2.4600

Moments

Mean 2.466

Std Dev $\quad 0.0054772$

Std Err Mean 0.0024495

upper $95 \%$ Mean 2.4728009

lower 95\% Mean 2.4591991

$\mathrm{N}$
Exhibit A10ac.

Distributions Type $=$ Simulated

Sample Crosscheck Data 500X

Dilution, Simulant=SRAT Receipt,

Known Concentration $(\mathrm{ppm})=15$,

Anion=Formate, ICS-3000

Instrument $=\mathrm{M}-13$

Measurement (ppm)

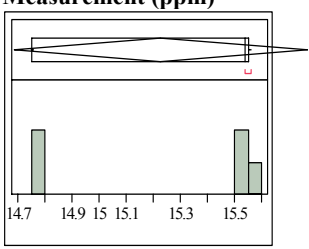

Quantiles

$100.0 \%$ maximum 15.560

$99.5 \% \quad 15.560$

$97.5 \% \quad 15.560$

$90.0 \% \quad 15.560$

$75.0 \%$ quartile $\quad 15.550$

$50.0 \%$ median

$25.0 \%$ quartile $\quad 14.750$

$10.0 \% \quad 14.750$

$2.5 \% \quad 14.750$

$0.5 \% \quad 14.750$

$0.0 \%$ minimum 14.750

Moments

Mean $\quad 15.228$

Std Dev $\quad 0.4364287$

Std Err Mean $\quad 0.1951768$

upper $95 \%$ Mean 15.769898

lower 95\% Mean 14.686102

$\mathrm{N}$

Instrument $=\mathrm{M}-14$

Measurement (ppm)

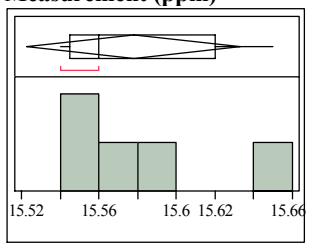

Quantiles

$100.0 \%$ maximum 15.650

$99.5 \% \quad 15.650$

$97.5 \% \quad 15.650$

$90.0 \% \quad 15.650$

$75.0 \%$ quartile $\quad 15.620$

$50.0 \%$ median $\quad 15.560$

$25.0 \%$ quartile $\quad 15.545$

$10.0 \% \quad 15.540$

$\begin{array}{ll}2.5 \% & 15.540\end{array}$

$0.5 \% \quad 15.540$

$0.0 \%$ minimum 15.540

Moments

Mean $\quad 15.578$

Std Dev $\quad 0.0443847$

Std Err Mean $\quad 0.0198494$

upper 95\% Mean 15.633111

lower $95 \%$ Mean 15.522889

$\mathrm{N}$
Exhibit A10ad.

Distributions Type=Simulated

Sample Crosscheck Data 500X

Dilution, Simulant=SRAT Receipt,

Known Concentration $(\mathrm{ppm})=15$,

Anion=Nitrate, ICS-3000

Instrument $=\mathbf{M}-13$

Measurement (ppm)

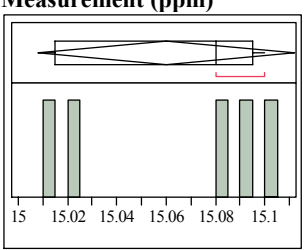

Quantiles

$100.0 \%$ maximum 15.100

$99.5 \% \quad 15.100$

$97.5 \% \quad 15.100$

$90.0 \% \quad 15.100$

$75.0 \%$ quartile 15.095

$50.0 \%$ median 15.080

$25.0 \%$ quartile $\quad 15.015$

$\begin{array}{ll}10.0 \% & 15.010\end{array}$

$2.5 \% \quad 15.010$

$0.5 \% \quad 15.010$

$0.0 \%$ minimum 15.010

Moments

Mean

$\begin{array}{lr}\text { Std Dev } & 0.041833 \\ \text { Std Err Mean } & 0.0187083\end{array}$

upper 95\% Mean 15.111943

lower 95\% Mean 15.008057

$\mathrm{N}$

Instrument $=\mathrm{M}-14$

Measurement (ppm)

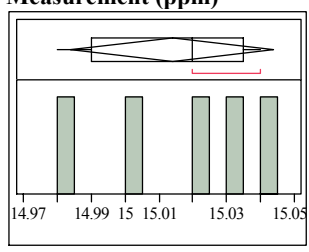

Quantiles

$100.0 \%$ maximum 15.040

$99.5 \% \quad 15.040$

$97.5 \% \quad 15.040$

$90.0 \% \quad 15.040$

$75.0 \%$ quartile 15.035

$50.0 \%$ median $\quad 15.020$

$25.0 \%$ quartile $\quad 14.990$

$10.0 \% \quad 14.980$

$2.5 \% \quad 14.980$

$0.5 \% \quad 14.980$

$0.0 \%$ minimum 14.980

Moments

Mean $\quad 15.014$

Std Dev $\quad 0.0240832$

Std Err Mean $\quad 0.0107703$

upper $95 \%$ Mean 15.043903

lower $95 \%$ Mean 14.984097

$\mathrm{N}$
Exhibit A10ae.

Distributions Type $=$ Simulated Sample Crosscheck Data 500X

Dilution, Simulant=SRAT Receipt,

Known Concentration (ppm) $=15$,

Anion=Nitrite, ICS-3000

Instrument $=\mathrm{M}-13$

Measurement (ppm)

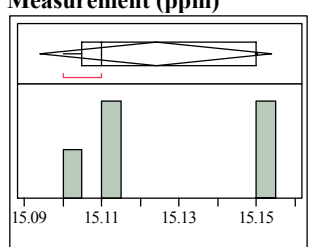

Quantiles

$100.0 \%$ maximum 15.150

$99.5 \% \quad 15.150$

$97.5 \%$

$90.0 \% \quad 15.150$

$75.0 \%$ quartile 15.150

$50.0 \%$ median 15.110

$25.0 \%$ quartile $\quad 15.105$

$10.0 \% \quad 15.100$

$2.5 \% \quad 15.100$

$0.5 \% \quad 15.100$

$0.0 \%$ minimum 15.100

Moments

Mean $\quad 15.124$

Std Dev $\quad 0.0240832$

Std Err Mean 0.0107703

upper 95\% Mean 15.153903

lower 95\% Mean 15.094097

N

Instrument $=\mathrm{M}-14$

Measurement (ppm)

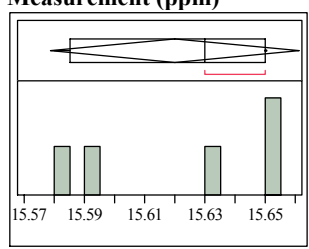

Quantiles

$100.0 \%$ maximum 15.650

$99.5 \% \quad 15.650$

$97.5 \% \quad 15.650$

$90.0 \% \quad 15.650$

$75.0 \%$ quartile $\quad 15.650$

$50.0 \%$ median 15.630

$25.0 \%$ quartile $\quad 15.585$

$10.0 \% \quad 15.580$

$2.5 \% \quad 15.580$

$0.5 \% \quad 15.580$

$0.0 \%$ minimum 15.580

Moments

Mean

Std Err Mean $\quad 0.0148324$

upper 95\% Mean 15.661181

lower 95\% Mean 15.578819

$\mathrm{N}$ 
Exhibit A11a.

Bivariate Fit of Measurement (ppm) By Known Concentration (ppm) Anion=Chloride, ICS-3000 Instrument $=$ M-13

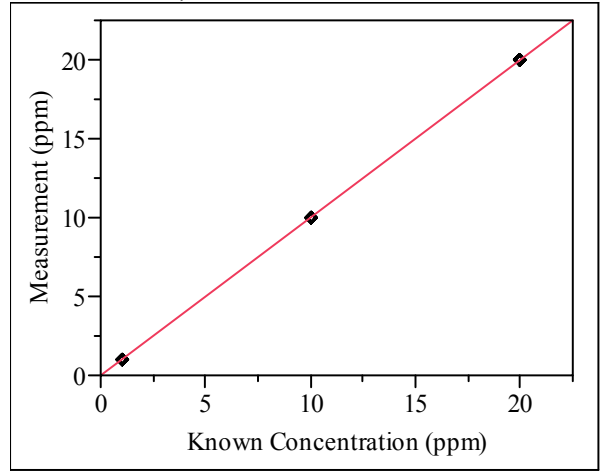

Linear Fit

Linear Fit

Measurement $(\mathrm{ppm})=-0.006956+1.0003506 *$ Known Concentration $(\mathrm{ppm})$

Summary of Fit

RSquare

RSquare Adj

0.999999

Root Mean Square Error $\quad 0.008729$

Mean of Response

0.33
10.33

Observations (or Sum Wgts) 9

Lack Of Fit

Source DF Sum of Squares Mean Square F Ratio

$\begin{array}{lllll}\text { Lack Of Fit } & 1 & 6.15013 \mathrm{e}-8 & 6.15 \mathrm{e}-8 & 0.0007\end{array}$

$\begin{array}{lllll}\text { Pure Error } & 6 & 0.00053333 & 0.000089 & \text { Prob }>\text { F }\end{array}$

Total Error $7 \quad 0.00053339$

0.000089 Prob $>$ F
0.9799

$\operatorname{Max}$ RSq

1.0000

Analysis of Variance

Source DF Sum of Squares Mean Square F Ratio

Model $\quad 1 \quad 542.38007 \quad 542.380 \quad 7117918$

$\begin{array}{llrr}\text { Error } & 7 & 0.00053 & 7.62 \mathrm{e}-5 \text { Prob }>\text { F }\end{array}$

$\begin{array}{llrr}\text { C. Total } & 8 & 542.38060 & <.0001\end{array}$

Parameter Estimates

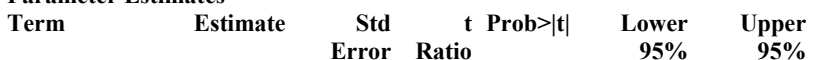

$\begin{array}{lllllll}\text { Intercept } & -0.006956 & 0.004845 & -1.44 & 0.1943 & -0.018413 & 0.0045019\end{array}$

$\begin{array}{lllllll}\text { Known } & 1.0003506 & 0.000375 & 2667.9 & <.0001 & 0.9994639 & 1.0012372\end{array}$

Concentration

(ppm)

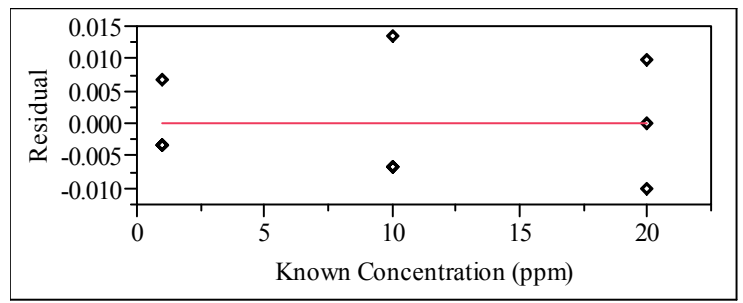

Exhibit A11b.

Bivariate Fit of Measurement (ppm) By Known Concentration (ppm) Anion $=$ Chloride, ICS-3000 Instrument $=$ M-14

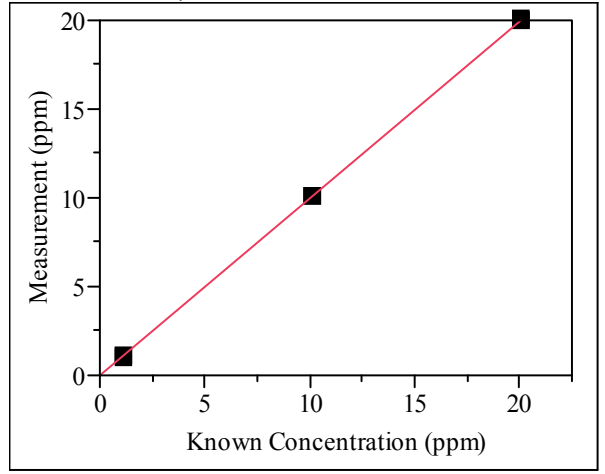

Linear Fit

Linear Fit

Measurement $(\mathrm{ppm})=-0.038309+0.9990836 *$ Known Concentration $(\mathrm{ppm})$

Summary of Fit

RSquare

RSquare Adj $\quad 0.999993$

Root Mean Square Error $\quad 0.021046$

Mean of Response $\quad 10.28556$

Observations (or Sum Wgts) 9

Lack Of Fit

Source DF Sum of Squares Mean Square F Ratio

$\begin{array}{lllll}\text { Lack Of Fit } & 1 & 0.00090043 & 0.000900 & 2.4557\end{array}$

$\begin{array}{lllll}\text { Pure Error } & 6 & 0.00220000 & 0.000367 & \text { Prob }>\text { F }\end{array}$

$\begin{array}{llrl}\text { Total Error } 7 & 0.00310043 & 0.1681\end{array}$

Max RSq

1.0000

Analysis of Variance

Source DF Sum of Squares Mean Square F Ratio

$\begin{array}{lllll}\text { Model } & 1 & 541.00712 & 541.007 & 1221459\end{array}$

$\begin{array}{llrr}\text { Error } & 7 & 0.00310 & 0.000443 \text { Prob }>\text { F }\end{array}$

$\begin{array}{llll}\text { C. Total } 8 & 541.01022 & <.0001\end{array}$

Parameter Estimates

Term Estimate Std t Prob $>|\mathrm{t}|$ Lower Upper

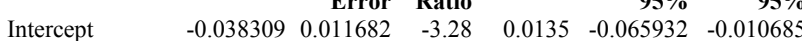

$\begin{array}{lllllll}\text { Known } & 0.9990836 & 0.000904 & 1105.2 & <.0001 & 0.9969461 & 1.0012212\end{array}$

Concentration

(ppm)

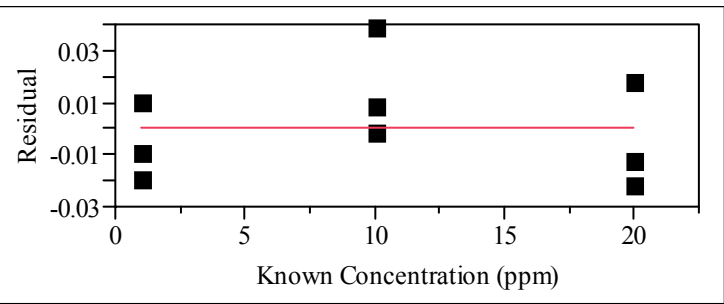


Exhibit A11c.

Bivariate Fit of Measurement (ppm) By Known Concentration (ppm) Anion=Fluoride, ICS-3000 Instrument $=$ M-13

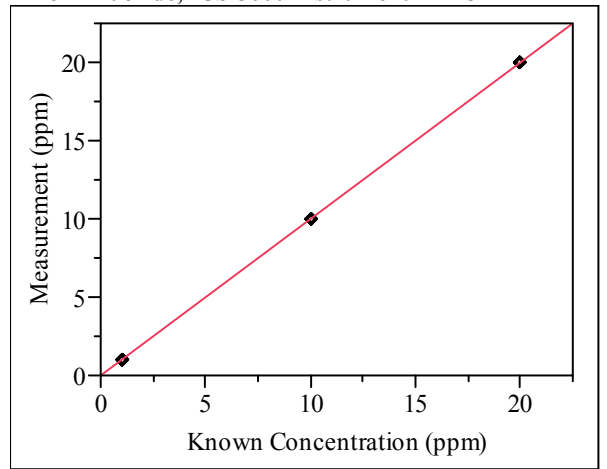

Linear Fit

Linear Fit

Measurement $(\mathrm{ppm})=0.0143604+0.9988253 *$ Known Concentration $(\mathrm{ppm})$

Summary of Fit

RSquare

RSquare Adj

0.999991

Root Mean Square Error $\quad 0.99999$

Mean of Response

0.025656

Observations (or Sum Wgts) 9

\section{Lack Of Fit}

$\begin{array}{lrrrr}\text { Source } & \text { DF } & \text { Sum of Squares } & \text { Mean Square } & \text { F Ratio } \\ \text { Lack Of Fit } & 1 & 0.00167435 & 0.001674 & 3.4248 \\ \text { Pure Error } & 6 & 0.00293333 & 0.000489 & \text { Prob }>\text { F } \\ \text { Total Error } & 7 & 0.00460769 & & 0.1137 \\ & & & & \text { Max RSq } \\ & & & 1.0000\end{array}$

Analysis of Variance

Source DF Sum of Squares Mean Square F Ratio

Model $\quad 1 \quad 540.72741 \quad 540.727 \quad 821473.2$

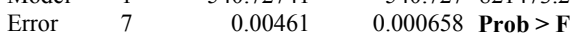

$\begin{array}{lrrr}\text { C. Total } 8 & 540.73202 & <.0001\end{array}$

Parameter Estimates

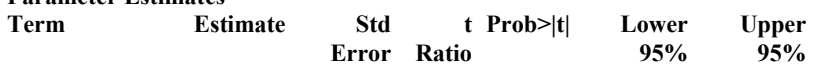

$\begin{array}{lllllll}\text { Intercept } & 0.0143604 & 0.014241 & 1.01 & 0.3469 & -0.019315 & 0.0480358\end{array}$ $\begin{array}{lllllll}\text { Known } & 0.9988253 & 0.001102 & 906.35 & <.0001 & 0.9962195 & 1.0014312\end{array}$

Concentration

(ppm)

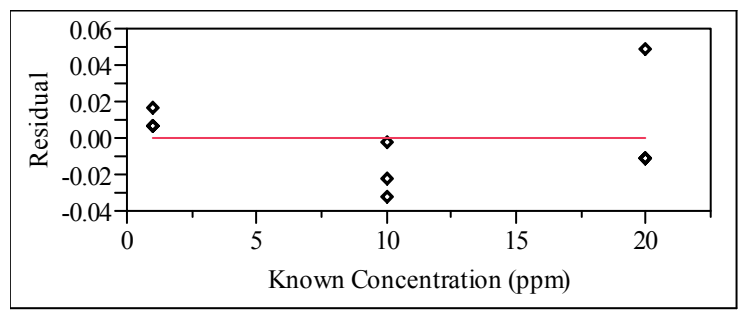

Exhibit A11d.

Bivariate Fit of Measurement (ppm) By Known Concentration (ppm) Anion=Fluoride, ICS-3000 Instrument $=$ M-14

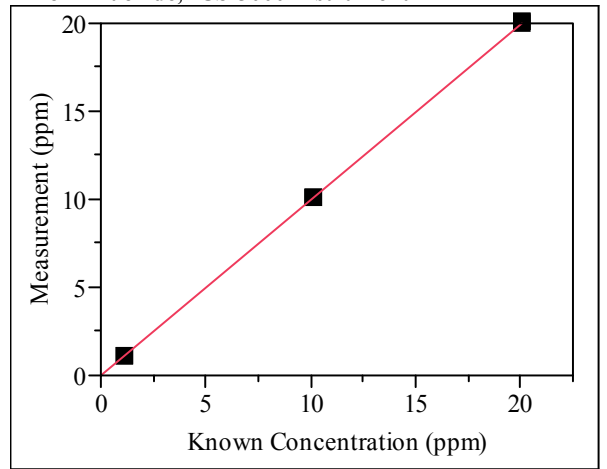

Linear Fit

Linear Fit

Measurement $(\mathrm{ppm})=0.0334133+0.9949385 *$ Known Concentration $(\mathrm{ppm})$

Summary of Fit

RSquare $\quad 0.999995$

RSquare Adj 0.999994

Root Mean Square Error $\quad 0.019285$

Mean of Response $\quad 10.31444$

Observations (or Sum Wgts) 9

Lack Of Fit

Source DF Sum of Squares Mean Square F Ratio

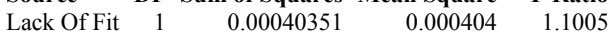

$\begin{array}{lllll}\text { Pure Error } & 6 & 0.00220000 & 0.000367 & \text { Prob }>\text { F }\end{array}$

$\begin{array}{llll}\text { Total Error } 7 & 0.00260351 & 0.3346\end{array}$

Max RSq

Analysis of Variance

Source DF Sum of Squares Mean Square F Ratio

$\begin{array}{lllll}\text { Model } & 1 & 536.52722 & 536.527 & 1442551\end{array}$

$\begin{array}{llrr}\text { Error } & 7 & 0.00260 & 0.000372 \text { Prob }>\text { F }\end{array}$

$\begin{array}{llrr}\text { C. Total } & 8 & 536.52982 & <.0001\end{array}$

Parameter Estimates

$\begin{array}{lrrrrr}\text { Term } & \text { Estimate } & \begin{array}{r}\text { Std } \\ \text { Error }\end{array} & \begin{array}{r}\text { t } \\ \text { Ratio }\end{array} & \begin{array}{r}\text { Power } \\ \text { 95\% }\end{array} & \begin{array}{r}\text { Upper } \\ \mathbf{9 5 \%}\end{array}\end{array}$

$\begin{array}{lllllll}\text { Intercept } & 0.0334133 & 0.010705 & 3.12 & 0.0168 & 0.0080999 & 0.0587267\end{array}$ $\begin{array}{lllllll}\text { Known } & 0.9949385 & 0.000828 & 1201.1 & <.0001 & 0.9929797 & 0.9968973\end{array}$

Concentration

(ppm)

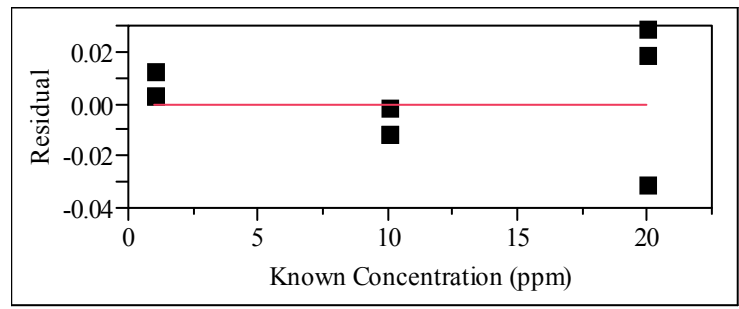


Exhibit A11e.

Bivariate Fit of Measurement (ppm) By Known Concentration (ppm) Anion=Formate, ICS-3000 Instrument $=$ M-13

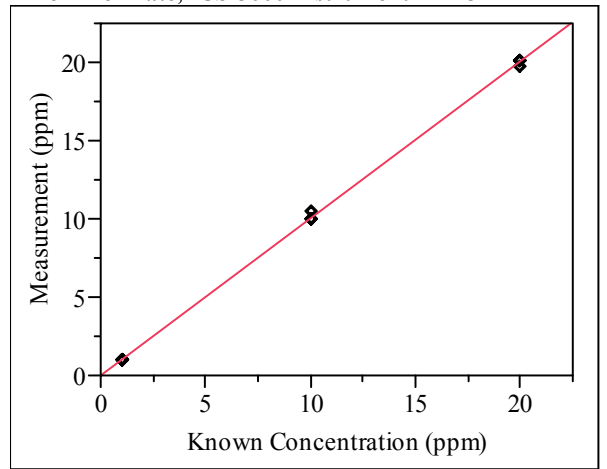

Linear Fit

Linear Fit

Measurement $(\mathrm{ppm})=0.0452829+1.0019619 *$ Known Concentration $(\mathrm{ppm})$

Summary of Fit

RSquare $\quad 0.999523$

$\begin{array}{ll}\text { RSquare Adj } & 0.999455 \\ \text { Root Mean Square Error } & 0.192553\end{array}$

Mean of Response $\quad 10.39889$

Observations (or Sum Wgts) 9

\section{Lack Of Fit}

$\begin{array}{lrrrr}\text { Source } & \text { DF } & \text { Sum of Squares } & \text { Mean Square } & \text { F Ratio } \\ \text { Lack Of Fit } & 1 & 0.05966943 & 0.059669 & 1.7913 \\ \text { Pure Error } & 6 & 0.19986667 & 0.033311 & \text { Prob }>\text { F } \\ \text { Total Error } & 7 & 0.25953610 & & 0.2293 \\ & & & & \text { Max RSq } \\ & & & 0.9996\end{array}$

Analysis of Variance

Source DF Sum of Squares Mean Square F Ratio

Model $\quad 1 \quad 544.12875 \quad 544.129 \quad 14675.81$

$\begin{array}{llrr}\text { Error } & 7 & 0.25954 & 0.037 \text { Prob }>\text { F }\end{array}$

$\begin{array}{lrrr}\text { C. Total } & 8 & 544.38829 & <.0001\end{array}$

Parameter Estimates

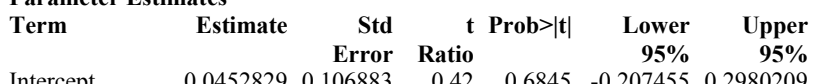

$\begin{array}{lllllll}\text { Intercept } & 0.0452829 & 0.106883 & 0.42 & 0.6845 & -0.207455 & 0.2980209\end{array}$ $\begin{array}{llllllll}\text { Known } & 1.0019619 & 0.008271 & 121.14 & <.0001 & 0.9824044 & 1.0215193\end{array}$

Concentration

(ppm)

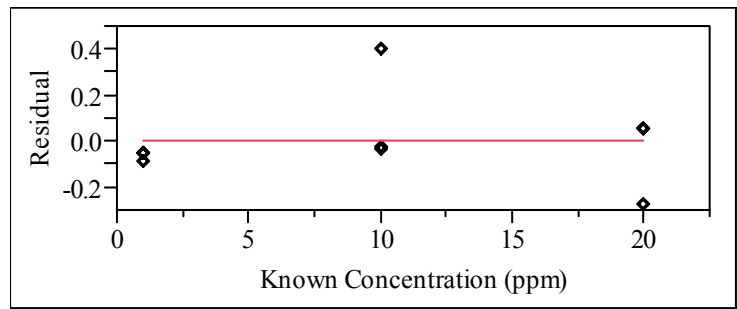

Exhibit A11f.

Bivariate Fit of Measurement (ppm) By Known Concentration (ppm) Anion=Formate, ICS-3000 Instrument $=$ M-14

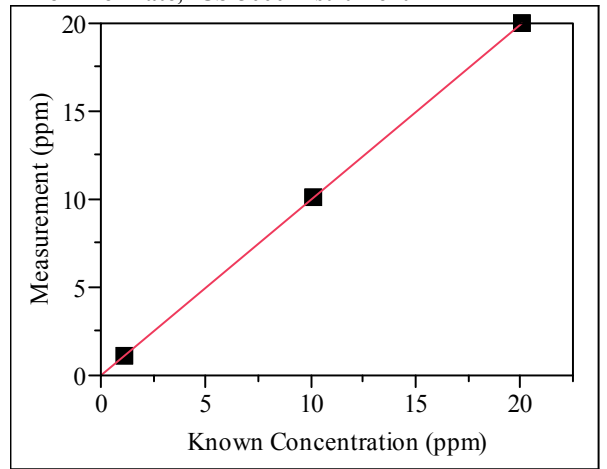

Linear Fit

Linear Fit

Measurement $(\mathrm{ppm})=0.0217343+0.9945633 *$ Known Concentration $(\mathrm{ppm})$

Summary of Fit

RSquare $\quad 0.999998$

RSquare Adj 0.999997

Root Mean Square Error $\quad 0.013105$

Mean of Response $\quad 10.29889$

Observations (or Sum Wgts) 9

Lack Of Fit

Source DF Sum of Squares Mean Square F Ratio

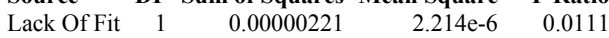

$\begin{array}{lllll}\text { Pure Error } & 6 & 0.00120000 & 0.000200 & \text { Prob }>\text { F }\end{array}$

$\begin{array}{llll}\text { Total Error } \quad 7 & 0.00120221 & 0.9196\end{array}$

Max RSq

Analysis of Variance

Source DF Sum of Squares Mean Square F Ratio

$\begin{array}{lllll}\text { Model } & 1 & 536.12269 & 536.123 & 3121623\end{array}$

$\begin{array}{llll}\text { Error } & 7 & 0.00120 & 0.000172 \quad \text { Prob }>\text { F }\end{array}$

$\begin{array}{llrr}\text { C. Total } & 8 & 536.12389 & <.0001\end{array}$

Parameter Estimates

$\begin{array}{lrrrrr}\text { Term } & \text { Estimate } & \begin{array}{r}\text { Std } \\ \text { Error }\end{array} & \begin{array}{r}\text { t } \\ \text { Ratio }\end{array} & \begin{array}{r}\text { Power }>|t| \\ \mathbf{9 5 \%}\end{array} & \begin{array}{r}\text { Upper } \\ \mathbf{9 5 \%}\end{array}\end{array}$

$\begin{array}{lllllll}\text { Intercept } & 0.0217343 & 0.007274 & 2.99 & 0.0203 & 0.004533 & 0.0389357\end{array}$ $\begin{array}{lllllll}\text { Known } & 0.9945633 & 0.000563 & 1766.8 & <.0001 & 0.9932323 & 0.9958944\end{array}$

Concentration

(ppm)

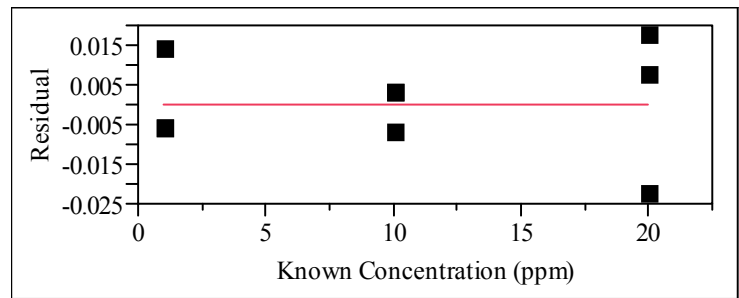




\section{Exhibit A11g.}

Bivariate Fit of Measurement (ppm) By Known Concentration (ppm) Anion=Nitrate, ICS-3000 Instrument $=$ M-13

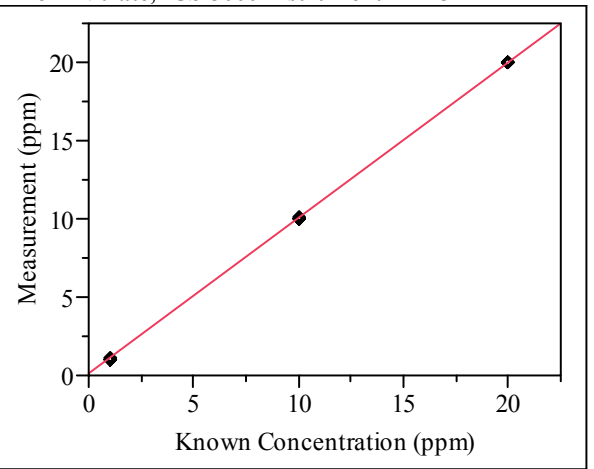

Linear Fit

Linear Fit

Measurement $(\mathrm{ppm})=0.062583+0.9987823 *$ Known Concentration $(\mathrm{ppm})$

Summary of Fit

RSquare

RSquare Adj

0.999997

Root Mean Square Error $\quad 0.999996$

Mean of Response

0.016019

Observations (or Sum Wgts) 9

\section{Lack Of Fit}

$\begin{array}{lrrrr}\text { Source } & \text { DF } & \text { Sum of Squares } & \text { Mean Square } & \text { F Ratio } \\ \text { Lack Of Fit } & 1 & 0.00006298 & 0.000063 & 0.2180 \\ \text { Pure Error } & 6 & 0.00173333 & 0.000289 & \text { Prob }>\text { F } \\ \text { Total Error } & 7 & 0.00179631 & & 0.6570 \\ & & & & \begin{array}{r}\text { Max RSq } \\ 1.0000\end{array}\end{array}$

Analysis of Variance

Source DF Sum of Squares Mean Square F Ratio

Model $\quad 1 \quad 540.68080 \quad 540.6812106967$

$\begin{array}{llrrr}\text { Error } & 7 & 0.00180 & 0.000257 & \text { Prob }>\text { F }\end{array}$

$\begin{array}{lrrr}\text { C. Total } & 8 & 540.68260 & <.0001\end{array}$

Parameter Estimates

$\begin{array}{lrrrrrr}\text { Term } & \text { Estimate } & \begin{array}{r}\text { Std } \\ \text { Error }\end{array} & \begin{array}{r}\text { t } \\ \text { Ratio }\end{array} & \text { Prob }>|\mathbf{t}| & \begin{array}{r}\text { Lower } \\ \mathbf{9 5 \%}\end{array} & \begin{array}{r}\text { Upper } \\ \mathbf{9 5 \%}\end{array} \\ \text { Intercept } & 0.062583 & 0.008892 & 7.04 & 0.0002 & 0.0415568 & 0.0836093\end{array}$

Known $\begin{array}{lllllll}0.9987823 & 0.000688 & 1451.5 & <.0001 & 0.9971552 & 1.0004093\end{array}$

Concentration

(ppm)

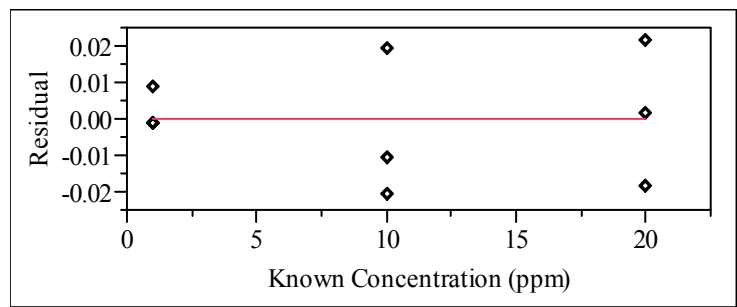

Exhibit A11h.

Bivariate Fit of Measurement (ppm) By Known Concentration (ppm) Anion=Nitrate, ICS-3000 Instrument=M-14

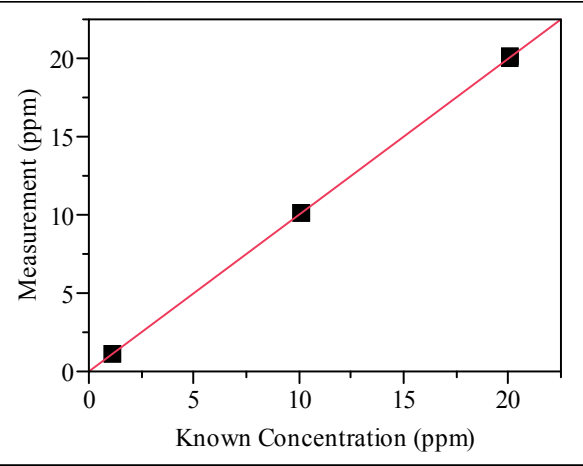

\section{Linear Fit}

Linear Fit

Measurement $(\mathrm{ppm})=0.0058979+0.9973862 *$ Known Concentration $(\mathrm{ppm})$

Summary of Fit

RSquare $\quad 0.999986$

RSquare Adj 0.999983

Root Mean Square Error $\quad 0.033351$

Mean of Response $\quad 10.31222$

Observations (or Sum Wgts) 9

Lack Of Fit

Source DF Sum of Squares Mean Square F Ratio

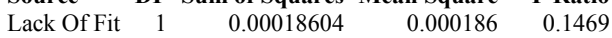

$\begin{array}{lllll}\text { Pure Error } & 6 & 0.00760000 & 0.001267 & \text { Prob }>\text { F }\end{array}$

$\begin{array}{llll}\text { Total Error } & 7 & 0.00778604 & 0.7148\end{array}$

Max RSq

Analysis of Variance

Source DF Sum of Squares Mean Square F Ratio

$\begin{array}{lllll}\text { Model } & 1 & 539.17037 & 539.170 & 484738.4\end{array}$

$\begin{array}{llrr}\text { Error } & 7 & 0.00779 & 0.001112 \text { Prob }>\text { F }\end{array}$

$\begin{array}{llrr}\text { C. Total } & 8 & 539.17816 & 0.00112 \\ \end{array}$

Parameter Estimates

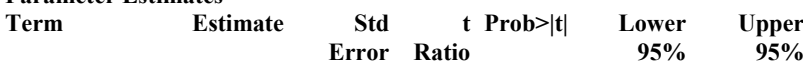

$\begin{array}{lllllll}\text { Intercept } & 0.0058979 & 0.018513 & 0.32 & 0.7593 & -0.037877 & 0.0496733\end{array}$ $\begin{array}{lllllll}\text { Known } & 0.9973862 & 0.001433 & 696.23 & <.0001 & 0.9939988 & 1.0007737\end{array}$

Concentration

(ppm)

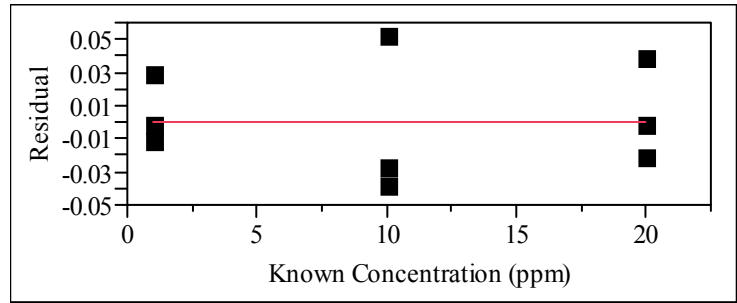


Exhibit A11i.

Bivariate Fit of Measurement (ppm) By Known Concentration (ppm)

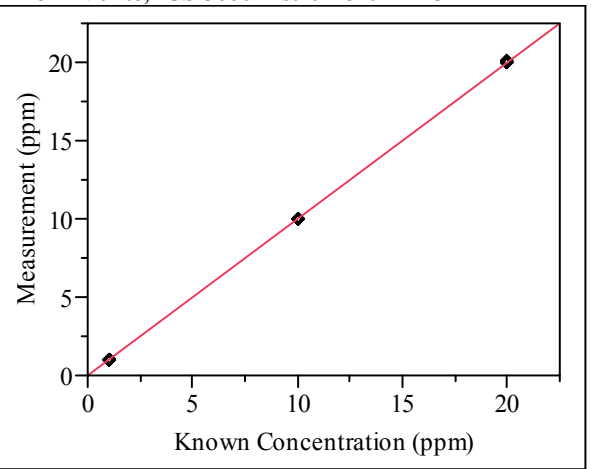

Linear Fit

Linear Fit

Measurement $(\mathrm{ppm})=0.0078598+1.0013899 *$ Known Concentration $(\mathrm{ppm})$

Summary of Fit

RSquare $\quad 0.999973$

$\begin{array}{lr}\text { RSquare Adj } & 0.999969 \\ \text { Root Mean Square Error } & 0.04615\end{array}$

Mean of Response $\quad 10.35556$

Observations (or Sum Wgts) 9

\section{Lack Of Fit}

Source DF Sum of Squares Mean Square F Ratio

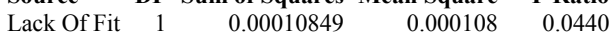

$\begin{array}{lllll}\text { Pure Error } & 6 & 0.01480000 & 0.002467 & \text { Prob }>\text { F }\end{array}$

Total Error $7 \quad 0.01490849$ Anion=Nitrite, ICS-3000 Instrument $=$ M-13

Exhibit A11j.

Bivariate Fit of Measurement (ppm) By Known Concentration (ppm) Anion=Nitrite, ICS-3000 Instrument=M-14

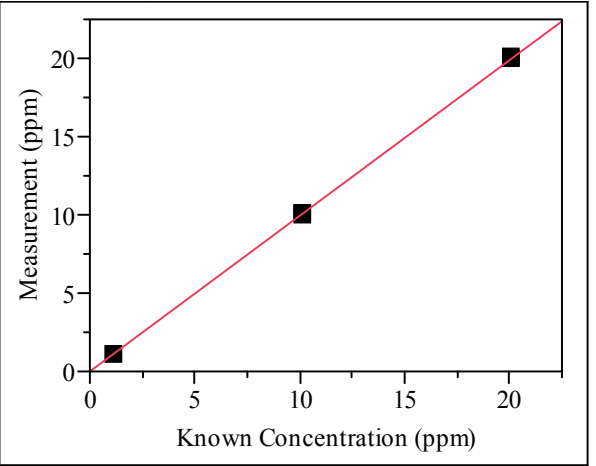

\section{Linear Fit}

Linear Fit

Measurement $(\mathrm{ppm})=-0.012374+0.9959287 *$ Known Concentration $(\mathrm{ppm})$

Summary of Fit

RSquare $\quad 0.999967$

RSquare Adj $\quad 0.999963$

Root Mean Square Error $\quad 0.050197$

Mean of Response $\quad 10.27889$

Observations (or Sum Wgts) 9

Lack Of Fit

Source DF Sum of Squares Mean Square F Ratio

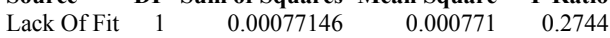

$\begin{array}{lllll}\text { Pure Error } & 6 & 0.01686667 & 0.002811 & \text { Prob }>\text { F }\end{array}$

$\begin{array}{llrl}\text { Total Error } & 7 & 0.01763813 & 0.6192\end{array}$

$\operatorname{Max} \mathbf{R S q}$

Analysis of Variance

Source DF Sum of Squares Mean Square F Ratio

Model $\quad 1 \quad 537.59565 \quad 537.596 \quad 213354.2$

$\begin{array}{llrr}\text { Error } & 7 & 0.01764 & 0.00252 \text { Prob }>\text { F }\end{array}$

$\begin{array}{llrr}\text { C. Total } & 8 & 537.61329 & <.0001\end{array}$

Parameter Estimates

Parameter Estimates

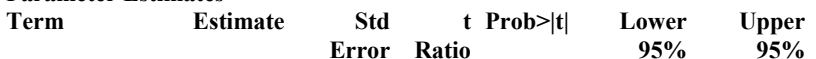

$\begin{array}{lllllll}\text { Intercept } & 0.0078598 & 0.025617 & 0.31 & 0.7679 & -0.052715 & 0.0684341\end{array}$ $\begin{array}{lllllll}\text { Known } & 1.0013899 & 0.001982 & 505.17 & <.0001 & 0.9967025 & 1.0060773\end{array}$

Concentration

(ppm)

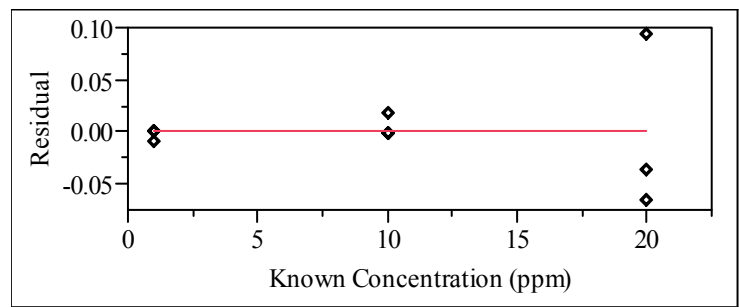

$\begin{array}{rrrrr}\text { Estimate } & \begin{array}{r}\text { Std } \\ \text { Error }\end{array} & \begin{array}{r}\text { t } \\ \text { Ratio }\end{array} & \begin{array}{r}\text { Lower } \\ \mathbf{9 5 \%}\end{array} & \begin{array}{r}\text { Upper } \\ \mathbf{9 5 \%}\end{array}\end{array}$ $\begin{array}{lllllll}-0.012374 & 0.027864 & -0.44 & 0.6704 & -0.078261 & 0.0535128\end{array}$ $\begin{array}{lllllll}0.9959287 & 0.002156 & 461.90 & <.0001 & 0.9908302 & 1.0010271\end{array}$

Intercept

Known

Concentration

(ppm)

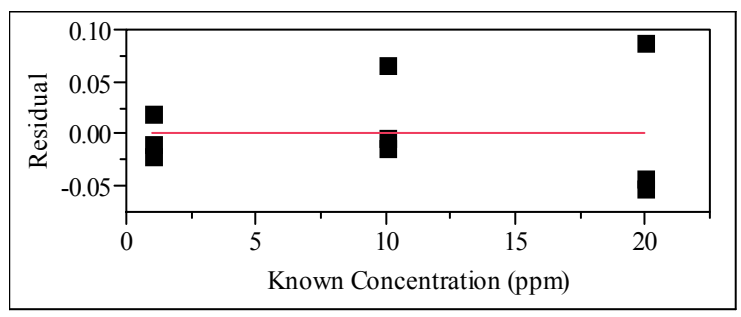


Exhibit A11k.

Bivariate Fit of Measurement (ppm) By Known Concentration (ppm) Anion=Oxalate, ICS-3000 Instrument $=$ M-13

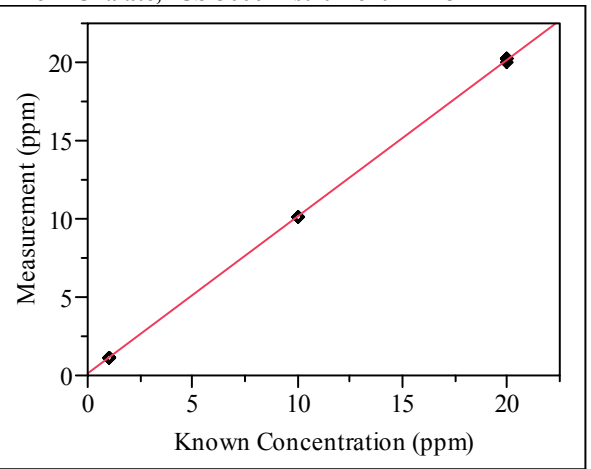

Linear Fit

Linear Fit

Measurement $(\mathrm{ppm})=0.0711931+1.0047232 *$ Known Concentration $(\mathrm{ppm})$

Summary of Fit

RSquare $\quad 0.999967$

RSquare Adj $\quad 0.999963$

Root Mean Square Error $\quad 0.05058$

Mean of Response $\quad 10.45333$

Observations (or Sum Wgts) 9

\section{Lack Of Fit}

Source DF Sum of Squares Mean Square F Ratio

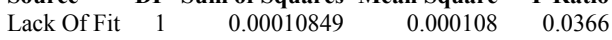

$\begin{array}{lllll}\text { Pure Error } & 6 & 0.01780000 & 0.002967 & \text { Prob }>\text { F }\end{array}$

Total Error $7 \quad 0.01790849$

0.8547

Max RSq

Analysis of Variance

Source DF Sum of Squares Mean Square F Ratio

$\begin{array}{lllll}\text { Model } & 1 & 547.13209 & 547.132 & 213860.9\end{array}$

$\begin{array}{llrrr}\text { Error } & 7 & 0.01791 & 0.002558 & \text { Prob }>\text { F }\end{array}$

$\begin{array}{lrrr}\text { C. Total } & 8 & 547.15000 & <.0001\end{array}$

Parameter Estimates

$\begin{array}{lrrrrrr}\text { Term } & \text { Estimate } & \begin{array}{r}\text { Std } \\ \text { Error }\end{array} & \begin{array}{r}\text { t } \\ \text { Ratio }\end{array} & \text { Prob }>|\mathbf{t}| & \begin{array}{r}\text { Lower } \\ \mathbf{9 5 \%}\end{array} & \begin{array}{r}\text { Upper } \\ \mathbf{9 5 \%}\end{array} \\ \text { Intercept } & 0.0711931 & 0.028076 & 2.54 & 0.0389 & 0.0048033 & 0.1375829\end{array}$

$\begin{array}{llllllll}\text { Known } & 1.0047232 & 0.002173 & 462.45 & <.0001 & 0.9995859 & 1.0098606\end{array}$

Concentration

(ppm)

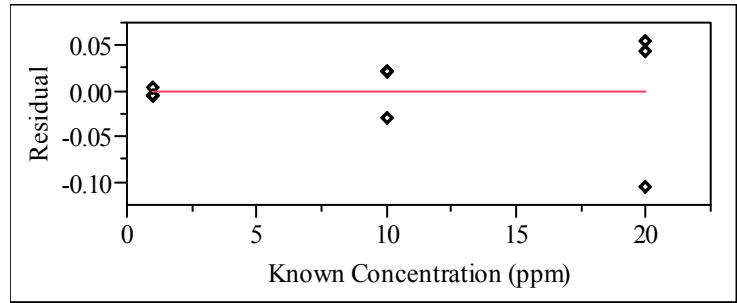

Exhibit A111.

Bivariate Fit of Measurement (ppm) By Known Concentration (ppm) Anion=Oxalate, ICS-3000 Instrument $=$ M-14

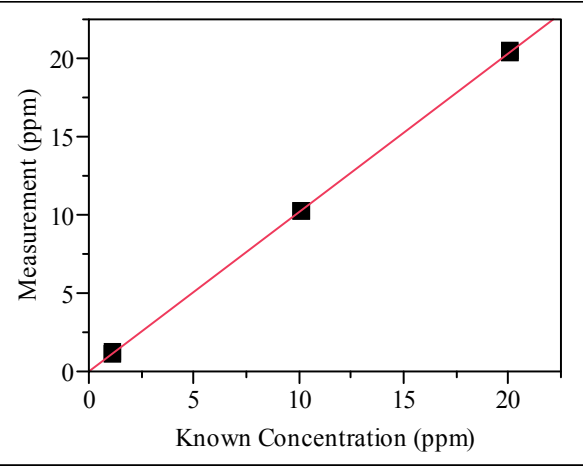

\section{Linear Fit}

Linear Fit

Measurement $(\mathrm{ppm})=0.0370172+1.0131919 *$ Known Concentration $(\mathrm{ppm})$

Summary of Fit

RSquare

RSquare Adj $\quad 0.999989$

Root Mean Square Error $\quad 0.027975$

Mean of Response $\quad 10.50667$

Observations (or Sum Wgts) 9

Lack Of Fit

Source DF Sum of Squares Mean Square F Ratio

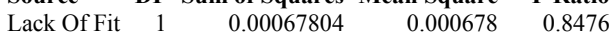

$\begin{array}{lllll}\text { Pure Error } & 6 & 0.00480000 & 0.000800 & \text { Prob }>\text { F }\end{array}$

$\begin{array}{llrl}\text { Total Error } & 7 & 0.00547804 & 0.3928\end{array}$

Max RSq

Analysis of Variance

Source DF Sum of Squares Mean Square F Ratio

$\begin{array}{lllll}\text { Model } & 1 & 556.39432 & 556.394 & 710976.4\end{array}$

$\begin{array}{llrr}\text { Error } & 7 & 0.00548 & 0.000783 \text { Prob }>\text { F }\end{array}$

$\begin{array}{llrr}\text { C. Total } & 8 & 556.39980 & <.0001\end{array}$

Parameter Estimates

$\begin{array}{lrrrrr}\text { Term } & \text { Estimate } & \begin{array}{r}\text { Std } \\ \text { Error }\end{array} & \begin{array}{r}\text { t } \\ \text { Ratio }\end{array} & \begin{array}{r}\text { Power } \\ \text { 95\% }\end{array} & \begin{array}{r}\text { Upper } \\ \mathbf{9 5 \%}\end{array}\end{array}$

$\begin{array}{lllllll}\text { Intercept } & 0.0370172 & 0.015528 & 2.38 & 0.0486 & 0.0002988 & 0.0737357\end{array}$ $\begin{array}{lllllll}\text { Known } & 1.0131919 & 0.001202 & 843.19 & <.0001 & 1.0103505 & 1.0160332\end{array}$

Concentration

(ppm)

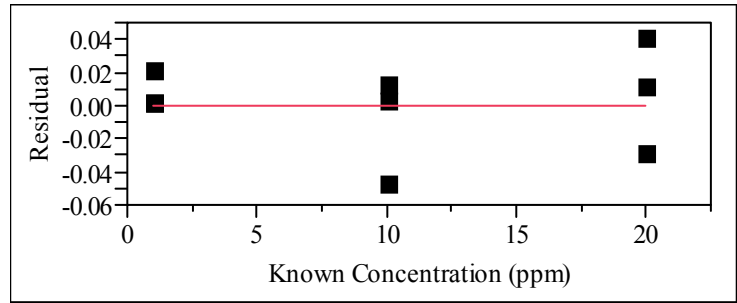


Exhibit A11m.

Bivariate Fit of Measurement (ppm) By Known Concentration (ppm) Anion $=$ Phosphate, ICS-3000 Instrument $=$ M-13

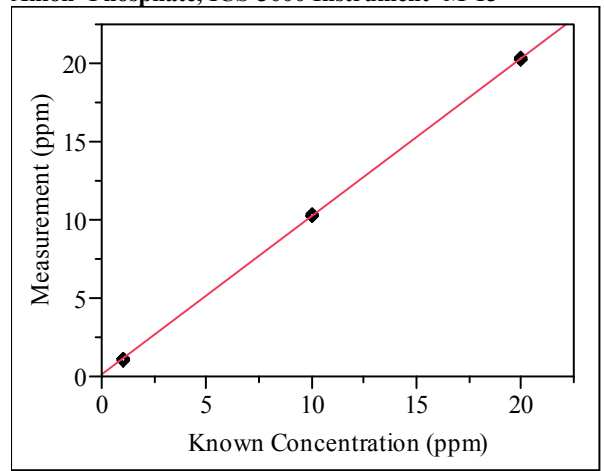

Linear Fit

Linear Fit

Measurement $(\mathrm{ppm})=0.1094588+1.0101599 *$ Known Concentration $(\mathrm{ppm})$

Summary of Fit

RSquare

RSquare Adj

0.999945

0.999937

Root Mean Square Error $\quad 0.065765$

Mean of Response

10.54778

Observations (or Sum Wgts)

9

Lack Of Fit

$\begin{array}{lrrrr}\text { Source } & \text { DF } & \text { Sum of Squares } & \text { Mean Square } & \text { F Ratio } \\ \text { Lack Of Fit } & 1 & 0.02140836 & 0.021408 & 14.4869 \\ \text { Pure Error } & 6 & 0.00886667 & 0.001478 & \text { Prob }>\text { F } \\ \text { Total Error } & 7 & 0.03027503 & & 0.0089 \\ & & & & \begin{array}{r}\text { Max RSq } \\ 1.0000\end{array}\end{array}$

Analysis of Variance

Source DF Sum of Squares Mean Square F Ratio

$\begin{array}{lllll}\text { Model } & 1 & 553.06928 & 553.069 & 127877.2\end{array}$

$\begin{array}{llrr}\text { Error } & 7 & 0.03028 & 0.004325 \text { Prob }>\text { F }\end{array}$

$\begin{array}{lrrr}\text { C. Total } 8 & 553.09956 & <.0001\end{array}$

Parameter Estimates

$\begin{array}{lrrrrrr}\text { Term } & \text { Estimate } & \begin{array}{r}\text { Std } \\ \text { Error }\end{array} & \begin{array}{r}\text { t } \\ \text { Ratio }\end{array} & \text { Prob }>|\mathbf{t}| & \begin{array}{r}\text { Lower } \\ \mathbf{9 5 \%}\end{array} & \begin{array}{r}\text { Upper } \\ \mathbf{9 5 \%}\end{array} \\ & & & 0\end{array}$

$\begin{array}{lllllll}\text { Intercept } & 0.1094588 & 0.036505 & 3.00 & 0.0200 & 0.0231383 & 0.1957793\end{array}$ $\begin{array}{lllllll}\text { Known } & 1.0101599 & 0.002825 & 357.60 & <.0001 & 1.0034802 & 1.0168396\end{array}$

Concentration

(ppm)

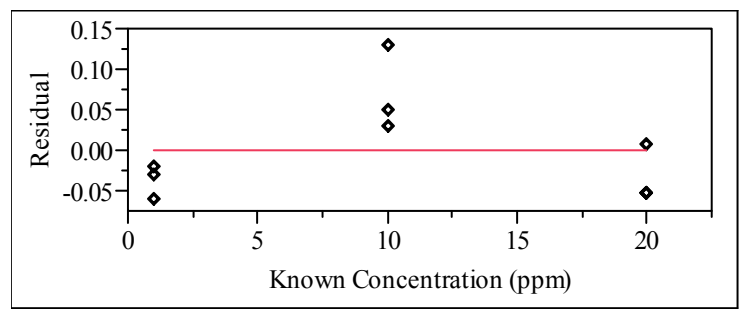

Exhibit A11n.

Bivariate Fit of Measurement (ppm) By Known Concentration (ppm) Anion=Phosphate, ICS-3000 Instrument $=$ M-14

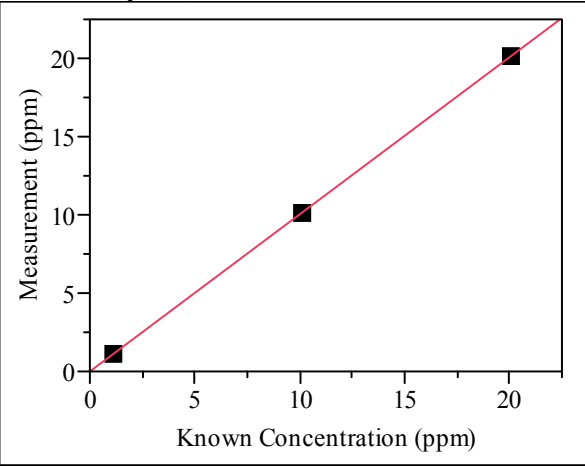

\section{Linear Fit}

Linear Fit

Measurement $(\mathrm{ppm})=-0.014569+1.0031304 *$ Known Concentration $(\mathrm{ppm})$

Summary of Fit

RSquare $\quad 0.999996$

RSquare Adj $\quad 0.999996$

Root Mean Square Error $\quad 0.017089$

$\begin{array}{lr}\text { Mean of Response } & 10.35111 \\ \text { Observations (or Sum Wgts) } & 9\end{array}$

Lack Of Fit

Source DF Sum of Squares Mean Square F Ratio

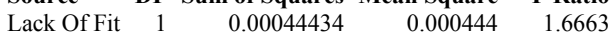

$\begin{array}{lllll}\text { Pure Error } & 6 & 0.00160000 & 0.000267 & \text { Prob }>\text { F }\end{array}$

$\begin{array}{llll}\text { Total Error } 7 & 0.00204434 & 0.2443\end{array}$

$\operatorname{Max} \mathbf{R S q}$

Analysis of Variance

Source DF Sum of Squares Mean Square F Ratio

Model $\quad 1 \quad 545.39864 \quad 545.399 \quad 1867491$

$\begin{array}{llrr}\text { Error } & 7 & 0.00204 & 0.000292 \text { Prob }>\text { F }\end{array}$

$\begin{array}{llll}\text { C. Total } & 8 & 545.40069 & <.0001\end{array}$

Parameter Estimates

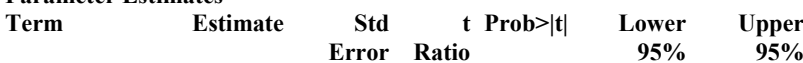

$\begin{array}{lllllll}\text { Intercept } & -0.014569 & 0.009486 & -1.54 & 0.1684 & -0.037 & 0.0078615\end{array}$ $\begin{array}{lllllll}\text { Known } & 1.0031304 & 0.000734 & 1366.6 & <.0001 & 1.0013946 & 1.0048661\end{array}$

Concentration

(ppm)

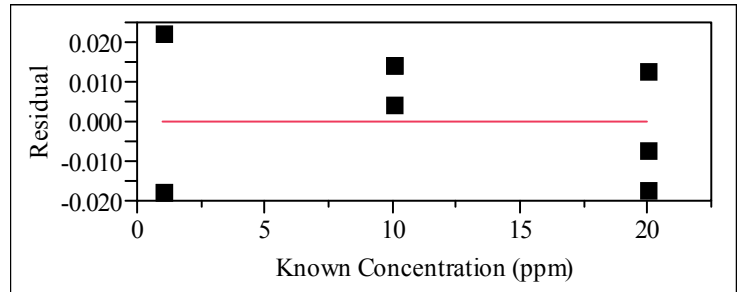


Exhibit A110.

Bivariate Fit of Measurement (ppm) By Known Concentration (ppm) Anion=Sulfate, ICS-3000 Instrument $=$ M-13

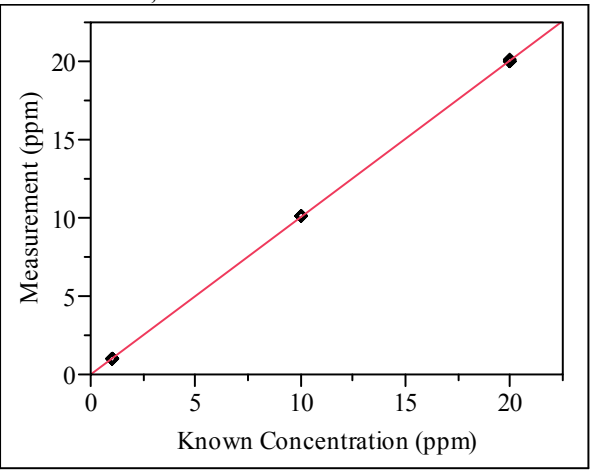

Linear Fit

Linear Fit

Measurement $(\mathrm{ppm})=0.0318204+1.0021894 *$ Known Concentration $(\mathrm{ppm})$

Summary of Fit

RSquare

RSquare Adj

0.999985

0.999982

Mean of Response

Observations (or Sum Wgts)

10.38778
9

\section{Lack Of Fit}

Source DF Sum of Squares Mean Square F Ratio

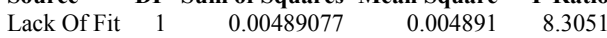

$\begin{array}{lllll}\text { Pure Error } & 6 & 0.00353333 & 0.000589 & \text { Prob }>\text { F }\end{array}$

Total Error $7 \quad 0.00842411$

Source DF Sum of Squares Mean Square F Ratio

Model $\quad 1 \quad 544.37593 \quad 544.376 \quad 452348.4$

$\begin{array}{llrr}\text { Error } & 7 & 0.00842 & 0.001203 \text { Prob }>\text { F }\end{array}$

$\begin{array}{lrrr}\text { C. Total } 8 & 544.38436 & <.0001\end{array}$

Parameter Estimates

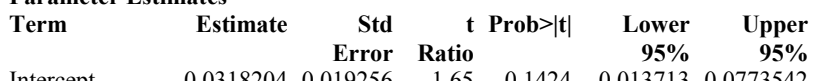

$\begin{array}{lllllll}\text { Intercept } & 0.0318204 & 0.019256 & 1.65 & 0.1424 & -0.013713 & 0.0773542\end{array}$ $\begin{array}{llllllll}\text { Known } & 1.0021894 & 0.00149 & 672.57 & <.0001 & 0.9986659 & 1.0057129\end{array}$

Concentration

(ppm)

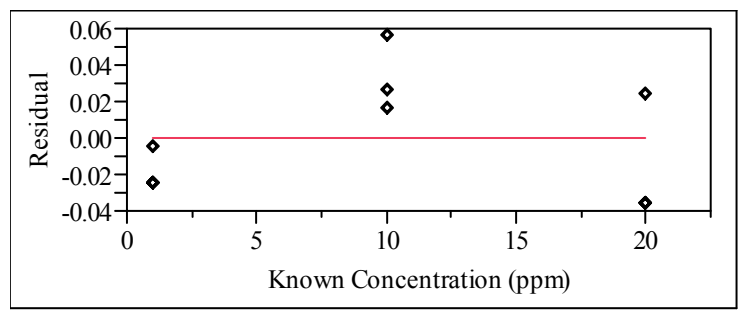

Exhibit A11p.

Bivariate Fit of Measurement (ppm) By Known Concentration (ppm) Anion=Sulfate, ICS-3000 Instrument $=$ M-14

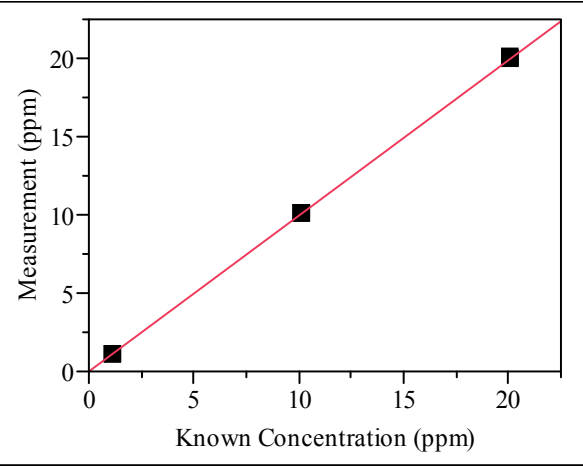

\section{Linear Fit}

Linear Fit

Measurement $(\mathrm{ppm})=0.0092435+0.9960947 *$ Known Concentration $(\mathrm{ppm})$

Summary of Fit

RSquare $\quad 0.999984$

RSquare Adj $\quad 0.999982$

Root Mean Square Error $\quad 0.034688$

Mean of Response $\quad 10.30222$

Observations (or Sum Wgts) 9

Lack Of Fit

Source DF Sum of Squares Mean Square F Ratio

$\begin{array}{lrrrr}\text { Lack Of Fit } & 1 & 0.00122269 & 0.001223 & 1.0189\end{array}$

$\begin{array}{lllll}\text { Pure Error } & 6 & 0.00720000 & 0.001200 & \text { Prob }>\text { F }\end{array}$

$\begin{array}{llll}\text { Total Error } \quad 7 & 0.00842269 & 0.3517\end{array}$

Max RSq

Analysis of Variance

Source DF Sum of Squares Mean Square F Ratio

Model $1 \quad 537.77493 \quad 537.775 \quad 446938.3$

$\begin{array}{llrr}\text { Error } & 7 & 0.00842 & 0.001203 \text { Prob }>\text { F }\end{array}$

$\begin{array}{llrr}\text { C. Total } & 8 & 537.78336 & <.0001\end{array}$

Parameter Estimates

$\begin{array}{lrrrrr}\text { Term } & \text { Estimate } & \begin{array}{r}\text { Std } \\ \text { Error }\end{array} & \begin{array}{r}\text { t } \\ \text { Ratio }\end{array} & \begin{array}{r}\text { Power } \\ \mathbf{9 5 \%}\end{array} & \begin{array}{r}\text { Upper } \\ \mathbf{9 5 \%}\end{array}\end{array}$

$\begin{array}{lllllll}\text { Intercept } & 0.0092435 & 0.019255 & 0.48 & 0.6458 & -0.036286 & 0.0547735\end{array}$ $\begin{array}{lllllll}\text { Known } & 0.9960947 & 0.00149 & 668.53 & <.0001 & 0.9925715 & 0.9996179\end{array}$

Concentration

(ppm)

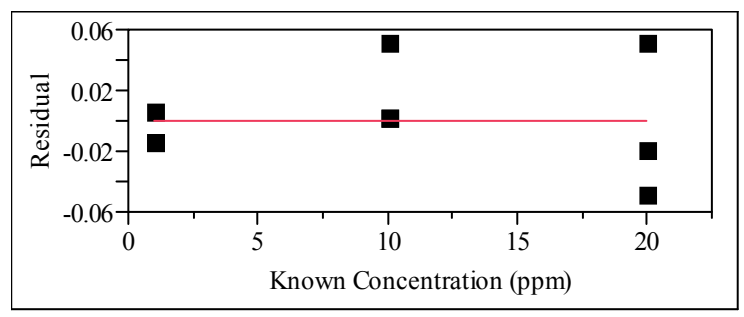


Exhibit A12a.

Oneway Analysis of Measurement (ppm) By Anion ICS-3000 Instrument $=$ M-13

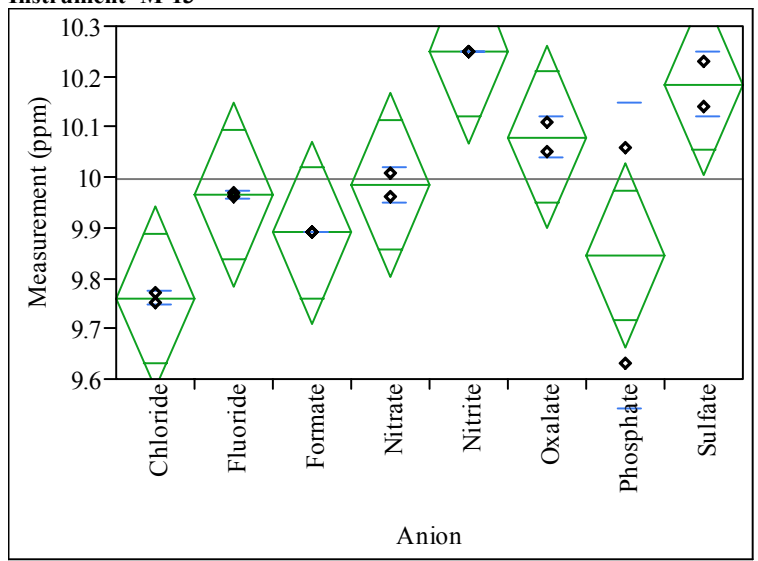

Oneway Anova

Summary of Fit

$\begin{array}{lr}\text { Rsquare } & 0.79879 \\ \text { Adj Rsquare } & 0.622732 \\ \text { Root Mean Square Error } & 0.111692 \\ \text { Mean of Response } & 9.995 \\ \text { Observations (or Sum Wgts) } & 16\end{array}$

Analysis of Variance

Source DF Sum of Squares Mean Square F Ratio Prob $>$ F

$\begin{array}{llllll}\text { Anion } & 7 & 0.39620000 & 0.056600 & 4.5371 & 0.0249\end{array}$

$\begin{array}{llll}\text { Error } & 8 & 0.09980000 & 0.012475\end{array}$

C. Total $15 \quad 0.49600000$

Means for Oneway Anova

Level Number Mean Std Error Lower 95\% Upper 95\%

$\begin{array}{lrrrrr}\text { Chloride } & 2 & 9.7600 & 0.07898 & 9.578 & 9.942\end{array}$

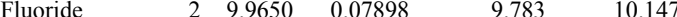

$\begin{array}{llllll}\text { Formate } & 2 & 9.8900 & 0.07898 & 9.708 & 10.072\end{array}$

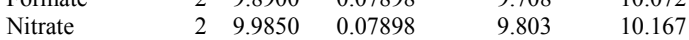

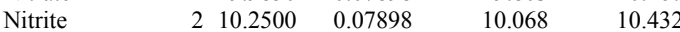

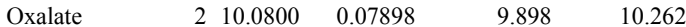

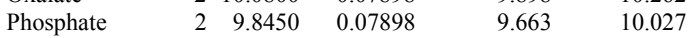

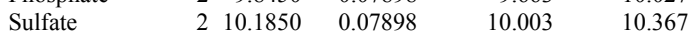

Std Error uses a pooled estimate of error variance
Exhibit A12b.

Oneway Analysis of Measurement (ppm) By Anion ICS-3000 Instrument=M-14

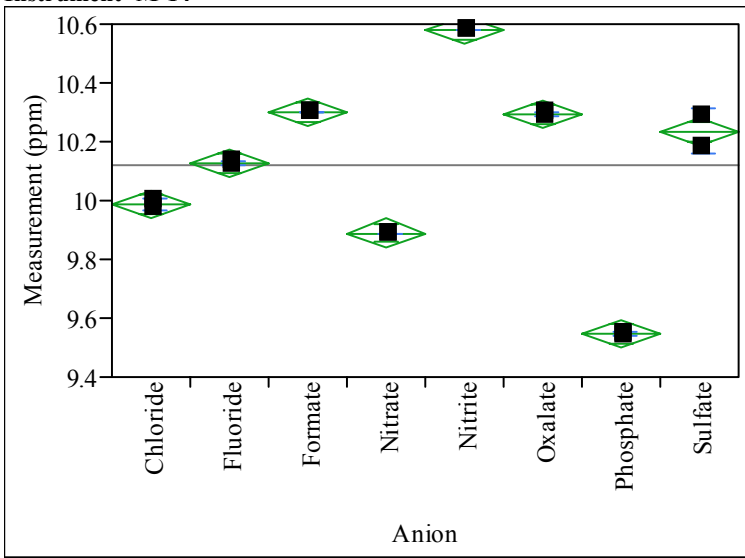

Oneway Anova

Summary of Fit

Rsquare

0.995202

Adj Rsquare

0.991003

Root Mean Square Error $\quad 0.028831$

Mean of Response $\quad 10.11938$

Observations (or Sum Wgts) $\quad 16$

Analysis of Variance

Source DF Sum of Squares Mean Square F Ratio Prob $>$ F

$\begin{array}{llllll}\text { Anion } & 7 & 1.3792438 & 0.197035 & 237.0344 & <.0001\end{array}$

$\begin{array}{llll}\text { Error } & 8 & 0.0066500 & 0.000831\end{array}$

C. Total $15 \quad 1.3858937$

Means for Oneway Anova

Level Number Mean Std Error Lower 95\% Upper 95\%

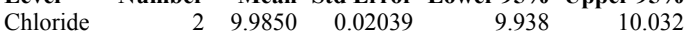

$\begin{array}{llllll}\text { Fluoride } & 2 & 10.1250 & 0.02039 & 10.078 & 10.172\end{array}$

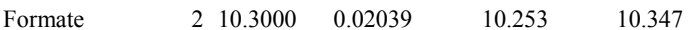

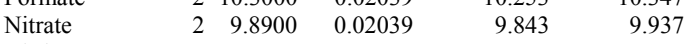

$\begin{array}{llllll}\text { Nitrite } & 2 & 10.5800 & 0.02039 & 10.533 & 10.627\end{array}$

$\begin{array}{llllll}\text { Oxalate } & 2 & 10.2950 & 0.02039 & 10.248 & 10.342\end{array}$

$\begin{array}{lrrrrr}\text { Phosphate } & 2 & 9.5450 & 0.02039 & 9.498 & 9.592\end{array}$

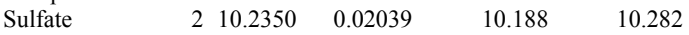

Std Error uses a pooled estimate of error variance 


\section{Distribution:}

C. J. Bannochie, 773-42A

D. R. Best, 999-W

J. M. Bricker, 704-27S

A. Y. Brown, 704-28S

C. J. Coleman, 773-A

T. B. Edwards, 999-W

M. T. Feller, 704-28S

T. L. Fellinger, 704-26S

C. C. Herman, 999-W

J. F. Iaukea, 704-30S

P. L. Lee, 773-42A

R. N. Mahannah, 773-28S

S. L. Marra, 773-A

R. T. McNew, 704-27S

J. E. Occhipinti, 704-S

A. B. Osteen, 704-S

D. K. Peeler, 999-W

J. W. Ray, 704-S

D. C. Sherburne, 704-S

M. E. Stone, 999-W

J. P. Vaughan, 773-41A 